\title{
Étienne Bonnot de CONDILLAC
}

\author{
(1715-1780) \\ Philosophe français \\ Abbé de Mureau
}

\section{TRAITÉ DES SENSATIONS}

\section{AUGEMENTÉ DE L'EXTRAIT RAISONNÉ}

\author{
Librairie Arthème Fayard, Paris, 1984. \\ Corpus des œuvres de philosophie en langue française
}

Un document produit en version numérique par Jean-Marc Simonet, bénévole,

Courriel : Jean-Marc_Simonet@uqac.ca
Dans le cadre de la collection: "Les classiques des sciences sociales"
Site web : http://classiques.uqac.ca/
Une collection développée en collaboration avec la Bibliothèque Paul-Émile-Boulet de l'Université du Québec à Chicoutimi
Site web : http://bibliotheque.uqac.ca/


Cette édition électronique a été réalisée par Jean-Marc Simonet, ancien professeur des Universités, bénévole.

Courriel : Jean-Marc_Simonet@uqac.ca

À partir du livre (fac simile de la Bibliothèque nationale de France) :

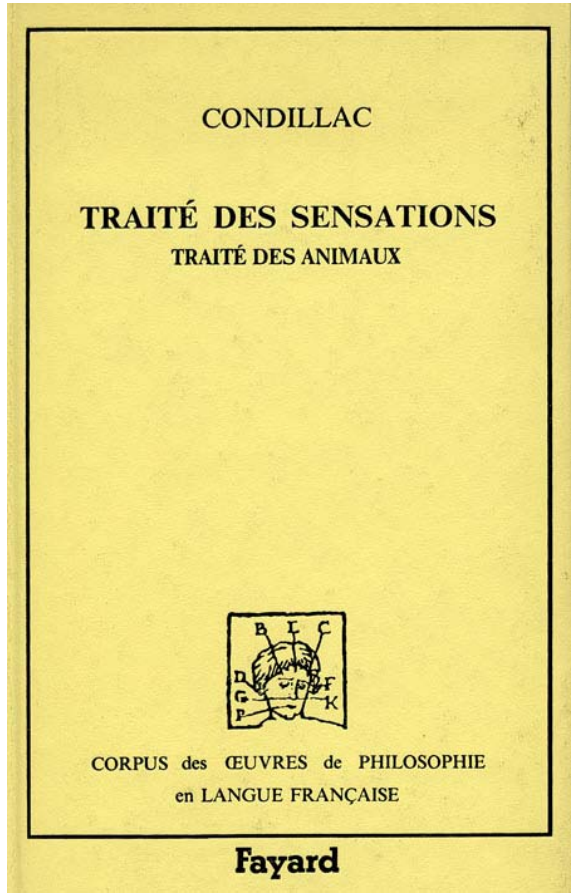

\section{Étienne Bonnot de} Condillac Philosophe français

(1715-1780)

\section{Traité des sensations}

Tiré des Euvres de Condillac, revues, corrigées par l'auteur, Ch. Houel, Imprimeur, Paris, 1798.

Corpus des œuvres de philosophie en langue française.

Librairie Arthème Fayard, Paris, 1984.

Polices de caractères utilisées :

Pour le texte: Times New Roman, 14 points.

Pour les notes de bas de page : Times New Roman, 12 points.

Édition électronique réalisée avec le traitement de textes Microsoft Word 2008 pour Macintosh.

Mise en page sur papier format : LETTRE (US letter), 8.5'” x 11’')

Édition numérique réalisée le 15 septembre 2010 à Chicoutimi, Ville de Saguenay, province de Québec, Canada 


\section{ÉTIENNE BONNOT DE CONDILLAC} 1715-1780

\section{TRAITÉ DES SENSATIONS}

\section{AUGMENTÉ DE L'EXTRAIT RAISONNÉ DU TRAITÉ DES SENSATIONS}

Texte revu et corrigé par l'auteur, conforme à l'édition posthume de 1798 


\title{
Table des matières
}

Avis important au lecteur

Dessein de cet ouvrage

\author{
PREMIÈRE PARTIE \\ Des sens qui, par eux-mêmes nejugent pas \\ des objets extérieurs.
}

Chapitre premier. Des premières connaissances d'un homme borné au sens de l'odorat.

Chapitre II. Des opérations de l'entendement dans un homme borné au sens de l'odorat, et comment les différents degrés de plaisir et de peine sont le principe de ces opérations.

Chapitre III. Des désirs, des passions, de l'amour, de la haine, de l'espérance, de la crainte et de la volonté dans un homme borné au sens de l'odorat.

Chapitre IV. Des idées d'un homme borné au sens de l'odorat.

Chapitre V. Du sommeil et des songes d'un homme borné à l'odorat.

Chapitre VI. Du moi, ou de la personnalité d'un homme borné à l'odorat.

Chapitre VII. Conclusion des chapitres précédents.

Chapitre VIII. D'un homme borné au sens del'ouie.

Chapitre IX. De l'odorat et de l'ouïe réunis.

Chapitre X. Du goût seul, et du goût joint à l'odorat et à l'ouie.

Chapitre XI. D'un homme bornéau sens de la vue.

Chapitre XII. De la vue avec l'odorat, l'ouie et le goût. 


\section{SECONDE PARTIE \\ Du toucher, ou du seul sens qui juge par lui-même des objets extérieurs.}

Chapitre premier. Du moindre degré de sentiment, où l'on peut réduire un homme borné au sens du toucher.

Chapitre II. Cet homme, borné au moindre degré de sentiment, n’a aucune idée d'étendue, ni de mouvement.

Chapitre III. Des sensations qu'on attribue au toucher et qui ne donnent cependant aucune idée d'étendue.

Chapitre IV. Considérations préliminaires à la solution de la question : Comment nous passons de nos sensations à ma connaissance des corps.

Chapitre V. Comment un homme borné au toucher découvre son corps et apprend qu'il y a quelque chose hors de lui.

Chapitre VI. Du plaisir, de la douleur, des besoins, et des désirs dans un homme borné au sens du toucher.

Chapitre VII. De la manière dont un homme borné au sens du toucher, commence à découvrir l'espace.

Chapitre VIII. Des idées que peut acquérir un homme borné au sens du toucher.

Chapitre IX. Observations propres à faciliter l'intelligence de ce qui sera dit en traitant de la vue.

Chapitre X. Du repos, du sommeil, et du réveil dans un homme borné au sens du toucher.

Chapitre XI. De la mémoire, de l'imagination et des songes dans un homme borné au sens du toucher.

Chapitre XII. Du principal organe du toucher. 


\section{TROISIĖME PARTIE. \\ Comment le toucher apprend aux autres sens \\ à juger des objets extérieurs.}

Chapitre premier. Du toucher avec l'odorat.

Chapitre II. De l'ouïe, de l'odorat et du tact réunis.

Chapitre III. Comment l'œil apprend à voir la distance, la situation, la figure, la grandeur et le mouvement des corps.

Chapitre IV. Pourquoi on est porté à attribuer à la vue des idées qu'on ne doit qu'au toucher. Par quelle suite de réflexions on est parvenu à détruire ce préjugé.

Chapitre V. D'un aveugle-né, à qui les cataractes ont été abaissées.

Chapitre VI. Comment on pourrait observer un aveugle-né, à qui on abaisserait les cataractes.

Chapitre VII. Del'idée que la vuejointe au toucher donne de la durée.

Chapitre VIII. Comment la vue, ajoutée au toucher, donne quelque connaissance de la durée du sommeil, et apprend à distinguer l'état de songe de l'état de veille.

Chapitre IX. De la chaîne des connaissances, des abstractions et des désirs, lorsque la vue est ajoutée au toucher, à l'ouie et à l'odorat.

Chapitre X. Du goût réuni au toucher.

Chapitre XI. Observations générales sur la réunion des cinq sens.

\section{QUATRIÈE PARTIE}

Des besoins, de l'industrie et des idées d'un homme isolé qui jouit de tous ses sens.

Chapitre premier. Comment cet homme apprend à satisfaire à ses besoins avec choix.

Chapitre II. De l'état d'un homme abandonné à lui-même, et comment les accidents auxquels il est exposé, contribuent à son instruction. 
Chapitre III. Des jugements qu'un homme abandonné à lui-même peut porter de la bonté et dela beauté des choses.

Chapitre IV. Des jugements qu'un homme abandonné à lui-même peut porter des objets dont il dépend.

Chapitre V. De l'incertitude des jugements que nous portons sur l'existence des qualités sensibles.

Chapitre VI. Considérations sur les idées abstraites et générales, que peut acquérir un homme qui vit hors de toute société.

Chapitre VII. D'un homme trouvé dans les forêts de Lithuanie.

Chapitre VIII. D'un homme qui se souviendrait d'avoir reçu successivement l'usage de ses sens.

Chapitre IX. Conclusion.

Dissertation sur la liberté.

Réponse à un reproche.

EXTRAIT RAISONNÉ du Traité des sensations.

\section{Introduction}

Précis de la première partie

Précis de la seconde partie

Précis de la troisième partie

Précis de la quatrième partie 


\section{Avertissement à propos de cette édition électronique :}

Pour faciliter la lecture, et sans rien changer d'autre, nous nous sommes contentés de moderniser l'orthographe et la typographie.

Dans le cas particulier des noms propres, nous avons adopté l'orthographe actuelle, pour permettre au lecteur d'effectuer les recherches qu'il souhaite sur les personnes en question. Par exemple : Malebranche au lieu de Mallebranche, Molyneux pour Molineux ou Barclay pour Barclai. 
Table des matières

\section{Avis important au lecteur}

J'ai oublié de prévenir sur une chose que j'aurais dû dire, et peutêtre répéter dans plusieurs endroits de cet ouvrage ; mais je compte que l'aveu de cet oubli vaudra des répétitions, sans en avoir l'inconvénient. J'avertis donc qu'il est très important de se mettre exactement à la place de la statue que nous allons observer. Il faut commencer d'exister avec elle, n'avoir qu'un seul sens, quand elle n’en a qu'un ; n'acquérir que les idées qu'elle acquiert, ne contracter que les habitudes qu'elle contracte : en un mot, il faut n'être que ce qu'elle est. Elle ne jugera des choses comme nous, que quand elle aura tous nos sens et toute notre expérience ; et nous ne jugerons comme elle, que quand nous nous supposerons privés de tout ce qui lui manque. Je crois que les lecteurs, qui se mettront exactement à sa place, n'auront pas de peine à entendre cet ouvrage ; les autres m'opposeront des difficultés sans nombre.

On ne comprend point encore ce que c'est que la statue que je me propose d'observer ; et cet avertissement paraîtra sans doute déplacé : mais ce sera une raison de plus pour le remarquer, et pour s'en souvenir. 


\section{Dessein de cet ouvrage}

Nous ne saurions nous rappeler l'ignorance, dans laquelle nous sommes nés : c'est un état qui ne laisse point de traces après lui. Nous ne nous souvenons d'avoir ignoré, que ce que nous nous souvenons d'avoir appris ; et pour remarquer ce que nous apprenons, il faut déjà savoir quelque chose: il faut s'être senti avec quelques idées, pour observer qu'on se sent avec des idées qu'on n'avait pas. Cette mémoire réfléchie, qui nous rend aujourd'hui si sensible le passage d'une connaissance à une autre, ne saurait donc remonter jusqu'aux premières : elle les suppose au contraire, et c'est là l'origine de ce penchant que nous avons à les croire nées avec nous. Dire que nous avons appris à voir, à entendre, à goûter, à sentir, à toucher, paraît le paradoxe le plus étrange. Il semble que la nature nous a donné l'entier usage de nos sens, à l'instant même qu'elle les a formés ; et que nous nous en sommes toujours servis sans étude, parce qu'aujourd'hui nous ne sommes plus obligés de les étudier.

J'étais dans ces préjugés, lorsque je publiai mon Essai sur l'origine des connaissances humaines. Je n'avais pu en être retiré par les raisonnements de Locke sur un aveugle-né, à qui on donnerait le sens de la vue ; et je soutins contre ce philosophe, que l'œil juge naturellement des figures, des grandeurs, des situations et des distances.

Vous savez, Madame, à qui je dois les lumières, qui ont enfin dissipé mes préjugés : vous savez la part qu'a eu à cet ouvrage une personne qui vous était si chère, et qui était si digne de votre estime et de votre amitié ${ }^{1}$. C'est à sa mémoire que je le consacre, et je m'adresse à vous, pour jouir tout-à-la-fois et du plaisir de parler d'elle, et du cha-

\footnotetext{
C’est elle qui m’a conseillé l'épigraphe Ut potero, explicabo, etc.
} 
grin de la regretter. Puisse ce monument perpétuer le souvenir de votre amitié mutuelle, et de l'honneur que j'aurai eu d'avoir part à l'estime de l'une et de l'autre !

Mais pourrais-je ne pas m'attendre à ce succès, quand je songe combien ce traité est à elle ? Les vues les plus exactes et les plus fines qu'il renferme, sont dues à la justesse de son esprit et à la vivacité de son imagination; qualités qu'elle réunissait dans un point, où elles paraissent presque incompatibles. Elle sentit la nécessité de considérer séparément nos sens, de distinguer avec précision les idées que nous devons à chacun d'eux, et d'observer avec quels progrès ils s'instruisent, et comment ils se prêtent des secours mutuels.

Pour remplir cet objet, nous imaginâmes une statue organisée intérieurement comme nous, et animée d'un esprit privé de toute espèce d'idées. Nous supposâmes encore que l'extérieur tout de marbre ne lui permettait l'usage d'aucun de ses sens, et nous nous réservâmes la liberté de les ouvrir à notre choix aux différentes impressions dont ils sont susceptibles.

Nous crûmes devoir commencer par l'odorat, parce que c'est de tous les sens celui qui paraît contribuer le moins aux connaissances de l'esprit humain. Les autres furent ensuite l'objet de nos recherches, et après les avoir considérés séparément et ensemble, nous vîmes la statue devenir un animal capable de veiller à sa conservation.

Le principe qui détermine le développement de ses facultés, est simple ; les sensations mêmes le renferment : car toutes étant nécessairement agréables ou désagréables, la statue est intéressée à jouir des unes et à se dérober aux autres. Or, on se convaincra que cet intérêt suffit pour donner lieu aux opérations de l'entendement et de la volonté. Le jugement, la réflexion, les désirs, les passions, etc. Ne sont que la sensation même qui se transforme différemment ${ }^{2}$. C'est

Mais, dira-t-on, les bêtes ont des sensations, et cependant leur âme n’est pas capable des mêmes facultés que celle de l'homme. Cela est vrai, et la lecture de cet ouvrage en rendra la raison sensible. L'organe du tact est en elles moins parfait ; et par conséquent il ne saurait être pour elles la cause occasionnelle de toutes les opérations qui se remarquent en nous. Je dis la cause occasionnelle, parce que les sensations sont les modifications propres de l'âme, et que les organes n'en peuvent être que l'occasion. De là le philosophe doit conclure, conformément à ce que la foi enseigne, que l'âme des bêtes est d'un ordre 
pourquoi il nous a paru inutile de supposer que l'âme tient immédiatement de la nature toutes les facultés dont elle est douée. La nature nous donne des organes, pour nous avertir par le plaisir de ce que nous avons à rechercher, et par la douleur de ce que nous avons à fuir. Mais elle s'arrête là ; et elle laisse à l'expérience le soin de nous faire contracter des habitudes, et d'achever l'ouvrage qu'elle a commencé.

Cet objet est neuf, et il montre toute la simplicité des voies de l'auteur de la nature. Peut-on ne pas admirer, qu'il n'ait fallu que rendre l'homme sensible au plaisir et à la douleur, pour faire naître en lui des idées, des désirs, des habitudes et des talents de toute espèce ?

Il y a sans doute bien des difficultés à surmonter, pour développer tout ce système ; et j'ai souvent éprouvé combien une pareille entreprise était au-dessus de mes forces. Mademoiselle FERRAND m'a éclairé sur les principes, sur le plan et sur les moindres détails ; et j'en dois être d'autant plus reconnaissant, que son projet n'était ni de m'instruire, ni de faire un livre. Elle ne s'apercevait pas qu'elle devenait auteur, et elle n'avait d'autre dessein que de s'entretenir avec moi des choses auxquelles je prenais quelque intérêt. Aussi ne se prévenait-elle jamais pour ses sentiments; et si je les ai presque toujours préférés à ceux que j'avais d'abord, j'ai eu le plaisir de ne me rendre qu'à la lumière. Je l'estimais trop, pour les adopter par tout autre motif ; et elle-même, elle en eût été offensée. Cependant il m’arrivait si souvent de reconnaître la supériorité de ses vues, que mon aveu ne pouvait éviter d'être soupçonné de trop de complaisance. Elle m’en faisait quelquefois des reproches ; elle craignait, disait-elle, de gâter mon ouvrage; et examinant avec scrupule les opinions que j’abandonnais, elle eût voulu se convaincre, que ses critiques n’étaient pas fondées.

Si elle avait pris elle-même la plume, cet ouvrage prouverait mieux quels étaient ses talents. Mais elle avait une délicatesse, qui ne lui permettait seulement pas d'y penser. Contraint d'y applaudir, quand je considérais les motifs qui en étaient le principe ; je l'en blâmais aussi

essentiellement différent de celle de l'homme. Car serait-il de la sagesse de Dieu qu'un esprit capable de s'élever à des connaissances de toute espèce, de découvrir ses devoirs, de mériter et de démériter, fût assujetti à un corps qui n’occasionnerait en lui que les facultés nécessaires à la conservation de l'animal ? 
parce que je voyais dans ses conseils ce qu'elle aurait pu faire ellemême. Ce traité n'est donc malheureusement que le résultat des conversations que j'ai eues avec elle, et je crains bien de n'avoir pas toujours su présenter ses pensées dans leur vrai jour. Il est fâcheux qu'elle n’ait pas pu m’éclairer jusqu'au moment de l'impression ; je regrette surtout qu'il y ait deux ou trois questions, sur lesquelles nous n’ayons pas été entièrement d'accord.

La justice que je rends à Mademoiselle FERRAND, je n'oserais la lui rendre, si elle vivait encore. Uniquement jalouse de la gloire de ses amis, et regardant comme à eux tout ce qui pouvait en elle y contribuer ; elle n'aurait point reconnu la part qu'elle a à cet ouvrage, elle m'aurait défendu d'en faire l'aveu, et je lui aurais obéi. Mais aujourd'hui dois-je me refuser au plaisir de lui rendre cette justice ? C'est tout ce qui me reste dans la perte que j'ai faite d'un conseil sage, d'un critique éclairé, d'un ami sûr.

Vous le partagerez avec moi, ce plaisir, madame, vous qui la regretterez toute votre vie ; et c'est aussi avec vous que j'aime à parler d'elle. Toutes deux également estimables, vous aviez ce discernement qui démêle tout le prix d'un objet aimable, et sans lequel on ne sait point aimer. Vous connaissiez la raison, la vérité et le courage qui vous formaient l'une pour l'autre. Ces qualités serraient les nœuds de votre amitié, et vous trouviez toujours dans votre commerce cet enjouement, qui est le caractère des âmes vertueuses et sensibles.

Ce bonheur devait donc finir ; et dans ces moments qui devaient en être le terme, il fallait qu'il ne restât d'autre consolation à votre amie, que de n'avoir point à vous survivre. Je l'ai vue se croire en cela fort heureuse. C'était assez pour elle de vivre dans votre mémoire. Elle aimait à s'occuper de cette idée; mais elle eût voulu en écarter l'image de votre douleur. Entretenez-vous quelquefois de moi avec Madame De Vassé, me disait-elle, et que ce soit avec une sorte de plaisir. Elle savait qu'en effet la douleur n'est pas la seule marque des regrets ; et qu'en pareil cas, plus on trouve de plaisir à penser à un ami, plus on sent vivement la perte qu'on a faite.

Que je suis flatté, madame, qu'elle m'ait jugé digne de partager avec vous cette douleur et ce plaisir! Que je le suis de l'honneur que vous me faites de porter le même jugement ! Pouviez-vous l'une et 
l'autre me donner une plus grande preuve de votre estime et de votre amitié ? 


\title{
PREMIÈRE PARTIE
}

\author{
Des sens qui, par eux-mêmes \\ ne jugent pas des objets extérieurs
}

Table des matières

\section{Chapitre premier}

Des premières connaissances d'un homme

borné au sens de l'odorat

La statue bornée à l'odorat, ne peut connaître que des odeurs.

Les connaissances de notre statue, bornée au sens de l'odorat, ne peuvent s'étendre qu'à des odeurs. Elle ne peut pas plus avoir les idées d'étendue, de figure, ni de rien qui soit hors d'elle, ou hors de ses sensations, que celles de couleur, de son, de saveur.

Elle n'est par rapport à elle, que les odeurs qu'elle sent.

Si nous lui présentons une rose, elle sera par rapport à nous, une statue qui sent une rose ; mais par rapport à elle, elle ne sera que l'odeur même de cette fleur.

Elle sera donc odeur de rose, d'œillet, de jasmin, de violette, suivant les objets qui agiront sur son organe. En un mot, les odeurs ne sont à son égard que ses propres modifications ou manières d'être ; et 
elle ne saurait se croire autre chose, puisque ce sont les seules sensations dont elle est susceptible.

Elle n’a aucune idée de la matière.

Que les philosophes à qui il paraît si évident que tout est matériel, se mettent pour un moment à sa place ; et qu'ils imaginent comment ils pourraient soupçonner qu'il existe quelque chose, qui ressemble à ce que nous appelons matière.

On ne peut pas être plus borné dans ses connaissances.

On peut donc déjà se convaincre qu'il suffirait d'augmenter ou de diminuer le nombre des sens, pour nous faire porter des jugements tout différents de ceux, qui nous sont aujourd'hui si naturels, et notre statue bornée à l'odorat, peut nous donner une idée de la classe des êtres, dont les connaissances sont le moins étendues. 


\section{Chapitre II}

Des opérations de l'entendement dans un homme borné au sens de l'odorat, et comment les différents degrés de plaisir et de peine sont le principe de ces opérations

La statue est capable d'attention.

A la première odeur, la capacité de sentir de notre statue est toute entière à l'impression qui se fait sur son organe. Voilà ce que j'appelle attention.

\section{De jouissance et de souffrance.}

Dès cet instant elle commence à jouir ou à souffrir : car si la capacité de sentir est toute entière à une odeur agréable, c'est jouissance ; et si elle est toute entière à une odeur désagréable, c’est souffrance.

Mais sans pouvoir former des désirs.

Mais notre statue n'a encore aucune idée des différents changements qu'elle pourra essuyer. Elle est donc bien, sans souhaiter d'être mieux ; ou mal, sans souhaiter d'être bien. La souffrance ne peut pas plus lui faire désirer un bien qu'elle ne connaît pas, que la jouissance lui faire craindre un mal qu'elle ne connaît pas davantage. Par conséquent, quelque désagréable que soit la première sensation, le fût-elle au point de blesser l'organe et d'être une douleur violente, elle ne saurait donner lieu au désir.

Si la souffrance est en nous toujours accompagnée du désir de ne pas souffrir, il ne peut pas en être de même de cette statue. La douleur est avant le désir d'un état différent, et elle n'occasionne en nous ce désir, que parce que cet état nous est déjà connu. L'habitude que nous avons contractée de la regarder comme une chose, sans laquelle nous 
avons été, et sans laquelle nous pouvons être encore, fait que nous ne pouvons plus souffrir, qu'aussitôt nous ne désirions de ne pas souffrir, et ce désir est inséparable d'un état douloureux.

Mais la statue qui, au premier instant, ne se sent que par la douleur même qu'elle éprouve, ignore si elle peut cesser d'être, pour devenir autre chose, ou pour n'être point du tout. Elle n'a encore aucune idée de changement, de succession, ni de durée. Elle existe donc sans pouvoir former des désirs.

\section{Plaisir et douleur, principes de ses opérations.}

Lorsqu'elle aura remarqué qu'elle peut cesser d'être ce qu'elle est, pour redevenir ce qu'elle a été, nous verrons ses désirs naître d'un état de douleur, qu'elle comparera à un état de plaisir que la mémoire lui rappellera. C'est par cet artifice que le plaisir et la douleur sont l'unique principe, qui, déterminant toutes les opérations de son âme, doit l'élever par degrés à toutes les connaissances, dont elle est capable; et pour démêler les progrès qu'elle pourra faire, il suffira d'observer les plaisirs qu'elle aura à désirer, les peines qu'elle aura à craindre, et l'influence des uns et des autres suivant les circonstances.

Combien elle serait bornée, si elle était sans mémoire.

S'il ne lui restait aucun souvenir de ses modifications, à chaque fois elle croirait sentir pour la première : des années entières viendraient se perdre dans chaque moment présent. Bornant donc toujours son attention à une seule manière d'être, jamais elle n'en comparerait deux ensemble, jamais elle ne jugerait de leurs rapports : elle jouirait ou souffrirait, sans avoir encore ni désir ni crainte.

\section{Naissance de la mémoire.}

Mais l'odeur qu'elle sent, ne lui échappe pas entièrement, aussitôt que le corps odoriférant cesse d'agir sur son organe. L'attention qu'elle lui a donnée, la retient encore ; et il en reste une impression plus ou moins forte, suivant que l'attention a été elle-même plus ou moins vive. Voilà la mémoire. 
Partage de la capacité de sentir entre l'odorat et la mémoire.

Lorsque notre statue est une nouvelle odeur, elle a donc encore présente celle qu'elle a été le moment précédent. Sa capacité de sentir se partage entre la mémoire et l'odorat; et la première de ces facultés est attentive à la sensation passée, tandis que la seconde est attentive à la sensation présente.

La mémoire n'est donc qu'une manière de sentir.

Il y a donc en elle deux manières de sentir, qui ne diffèrent, que parce que l'une se rapporte à une sensation actuelle, et l'autre à une sensation qui n'est plus ; mais dont l'impression dure encore. Ignorant qu'il y a des objets qui agissent sur elle, ignorant même qu'elle a un organe ; elle ne distingue ordinairement le souvenir d'une sensation d'avec une sensation actuelle, que comme sentir faiblement ce qu'elle a été, et sentir vivement ce qu'elle est.

Le sentiment peut en être plus vif que celui de la sensation.

Je dis ordinairement, parce que le souvenir ne sera pas toujours un sentiment faible, ni la sensation un sentiment vif. Car toutes les fois que la mémoire lui retracera ses manières d'être avec beaucoup de force, et que l'organe au contraire ne recevra que de légères impressions ; alors le sentiment d'une sensation actuelle sera bien moins vif, que le souvenir d'une sensation qui n'est plus.

\section{La statue distingue en elle une succession.}

Ainsi donc qu'une odeur est présente à l'odorat par l'impression d'un corps odoriférant sur l'organe même, une autre odeur est présente à la mémoire, parce que l'impression d'un autre corps odoriférant subsiste dans le cerveau, où l'organe l'a transmise. En passant de la sorte par deux manières d'être, la statue sent qu'elle n'est plus ce qu'elle a été : la connaissance de ce changement lui fait rapporter la première à un moment différent de celui où elle éprouve la seconde : et c'est là ce qui lui fait mettre de la différence entre exister d'une manière et se souvenir d'avoir existé d'une autre. 
Comment elle est active et passive.

Elle est active par rapport à l'une de ses manières de sentir, et passive par rapport à l'autre. Elle est active, lorsqu'elle se souvient d'une sensation, parce qu'elle a en elle la cause qui la lui rappelle, c'est-àdire, la mémoire. Elle est passive au moment qu'elle éprouve une sensation, parce que la cause qui la produit est hors d'elle, c'est-à-dire, dans les corps odoriférants qui agissent sur son organe ${ }^{3}$.

Elle ne peut pas faire la différence de ses deux

états.

Mais ne pouvant se douter de l'action des objets extérieurs sur elle, elle ne saurait faire la différence d'une cause qui est en elle, d'avec une cause qui est au-dehors. Toutes ses modifications sont à son égard, comme si elle ne les devait qu'à elle-même ; et soit qu'elle éprouve une sensation, ou qu'elle ne fasse que se la rappeler ; elle n’aperçoit jamais autre chose, sinon qu'elle est ou qu'elle a été de telle manière. Elle ne saurait, par conséquent, remarquer aucune différence entre l'état où elle est active, et celui où elle est toute passive.

\section{La mémoire devient en elle une habitude.}

Cependant plus la mémoire aura occasion de s'exercer, plus elle agira avec facilité. C'est par là que la statue se fera une habitude de se rappeler sans effort les changements par où elle a passé, et de partager son attention entre ce qu'elle est et ce qu'elle a été. Car une habitude n'est que la facilité de répéter ce qu'on a fait, et cette facilité s'acquiert par la réitération des actes ${ }^{4}$.

3 Il y a en nous un principe de nos actions, que nous sentons, mais que nous ne pouvons définir, on l'appelle force. Nous sommes également actifs par rapport à tout ce que cette force produit en nous, ou au-dehors. Nous le sommes, par exemple, lorsque nous réfléchissons, ou lorsque nous faisons mouvoir un corps. Par analogie nous supposons dans tous les objets qui produisent quelque changement, une force que nous connaissons encore moins; et nous sommes passifs par rapport aux impressions qu'ils font sur nous. Ainsi un être est actif ou passif, suivant que la cause de l'effet produit est en lui ou hors de lui.

4 Je ne parle ici, et dans tout cet ouvrage, que des habitudes qui s'acquièrent naturellement ; tout est soumis à d'autres lois dans l'ordre surnaturel. 
Elle compare.

Si après avoir senti à plusieurs reprises une rose et un œillet, elle sent encore une fois une rose; l'attention passive qui se fait par l'odorat, sera toute à l'odeur présente de rose, et l'attention active, qui se fait par la mémoire, sera partagée entre le souvenir qui reste des odeurs de rose et d'œillet. Or, les manières d'être ne peuvent se partager la capacité de sentir, qu'elles ne se comparent : car comparer n'est autre chose que donner en même temps son attention à deux idées.

Juge.

Dès qu'il y a comparaison, il y a jugement. Notre statue ne peut être en même temps attentive à l'odeur de rose et à celle d'œillet, sans apercevoir que l'une n'est pas l'autre ; et elle ne peut l'être à l'odeur d'une rose qu'elle sent, et à celle d'une rose qu'elle a sentie, sans apercevoir qu'elles sont une même modification. Un jugement n'est donc que la perception d'un rapport entre deux idées, que l'on compare.

Ces opérations tournent en habitude.

A mesure que les comparaisons et les jugements se répètent, notre statue les fait avec plus de facilité. Elle contracte donc l'habitude de comparer et de juger. Il suffira, par conséquent, de lui faire sentir d'autres odeurs, pour lui faire faire de nouvelles comparaisons, porter de nouveaux jugements, et contracter de nouvelles habitudes.

Elle devient capable d'étonnement.

Elle n'est point surprise à la première sensation qu'elle éprouve : car elle n'est encore accoutumée à aucune sorte de jugement.

Elle ne l'est pas non plus, lorsque, sentant successivement plusieurs odeurs, elle ne les aperçoit chacune qu'un instant. Alors elle ne tient à aucun des jugements qu'elle porte ; et plus elle change, plus elle doit se sentir naturellement portée à changer.

Elle ne le sera pas davantage, si par des nuances insensibles nous la conduisons de l'habitude de se croire une odeur, à juger qu'elle en est une autre : car elle change sans pouvoir le remarquer. 
Mais elle ne pourra manquer de l'être, si elle passe tout-à-coup d'un état auquel elle était accoutumée, à un état tout différent, dont elle n’avait point encore d'idée.

Cet étonnement donne plus d'activité aux opérations de l’âme.

Cet étonnement lui fait mieux sentir la différence de ses manières d'être. Plus le passage des unes aux autres est brusque, plus son étonnement est grand, et plus aussi elle est frappée du contraste des plaisirs et des peines qui les accompagnent. Son attention déterminée par des plaisirs et par des peines qui se font mieux sentir, s’applique avec plus de vivacité à toutes les sensations qui se succèdent. Elle les compare donc avec plus de soin : elle juge donc mieux de leurs rapports. L'étonnement augmente, par conséquent, l'activité des opérations de son âme. Mais puisqu'il ne l'augmente, qu'en faisant remarquer une opposition plus sensible entre les sentiments agréables et les sentiments désagréables, c'est toujours le plaisir et la douleur qui sont le premier mobile de ses facultés.

Idées qui se conservent dans la mémoire.

Si les odeurs attirent chacune également son attention, elles se conserveront dans sa mémoire, suivant l'ordre où elles se seront succédées, et elles s’y lieront par ce moyen.

Si la succession en renferme un grand nombre, l'impression des dernières, comme la plus nouvelle, sera la plus forte ; celle des premières s'affaiblira par des degrés insensibles, s'éteindra tout-à-fait, et elles seront comme non avenues.

Mais s'il y en a qui n'ont eu que peu de part à l'attention, elles ne laisseront aucune impression après elle, et elles seront aussitôt oubliées qu'aperçues.

Enfin, celles qui l'auront frappée davantage, se retraceront avec plus de vivacité, et l'occuperont si fort, qu'elles seront capables de lui faire oublier les autres. 
Liaison de ces idées.

La mémoire est donc une suite d'idées, qui forment une espèce de chaîne. C'est cette liaison qui fournit les moyens de passer d'une idée à une autre, et de se rappeler les plus éloignées. On ne se souvient, par conséquent, d'une idée qu'on a eue, il y a quelque temps, que parce qu'on se retrace avec plus ou moins de rapidité les idées intermédiaires.

Le plaisir conduit la mémoire.

A la seconde sensation, la mémoire de notre statue n'a pas de choix à faire : elle ne peut rappeler que la première. Elle agira seulement avec plus de force, suivant qu'elle y sera déterminée par la vivacité du plaisir et de la peine.

Mais lorsqu'il y a eu une suite de modifications, la statue conservant le souvenir d'un grand nombre, sera portée à se retracer préférablement celles qui peuvent davantage contribuer à son bonheur : elle passera rapidement sur les autres, ou ne s'y arrêtera que malgré elle.

Pour mettre cette vérité dans tout son jour, il faut connaître les différents degrés de plaisir et de peine, dont on peut être susceptible, et les comparaisons qu'on en peut faire.

Deux espèces de plaisirs et de peines.

Les plaisirs et les peines sont de deux espèces. Les uns appartiennent plus particulièrement au corps ; ils sont sensibles : les autres sont dans la mémoire et dans toutes les facultés de l'âme ; ils sont intellectuels ou spirituels. Mais c'est une différence que la statue est incapable de remarquer.

Cette ignorance la garantira d'une erreur, que nous avons de la peine à éviter : car ces sentiments ne diffèrent pas autant que nous l'imaginons. Dans le vrai, ils sont tous intellectuels ou spirituels, parce qu'il n'y a proprement que l'âme qui sente. Si l'on veut, ils sont aussi tous en un sens sensibles ou corporels, parce que le corps en est la seule cause occasionnelle. Ce n'est que suivant leur rapport aux fa- 
cultés du corps ou à celles de l'âme, que nous les distinguons en deux espèces.

Différents degrés dans l’un et dans l’autre.

Le plaisir peut diminuer ou augmenter par degrés ; en diminuant, il tend à s'éteindre, et il s'évanouit avec la sensation. En augmentant, au contraire, il peut conduire jusqu'à la douleur, parce que l'impression devient trop forte pour l'organe. Ainsi il y a deux termes dans le plaisir. Le plus faible est où la sensation commence avec le moins de force ; c'est le premier pas du néant au sentiment : le plus fort est où la sensation ne peut augmenter, sans cesser d'être agréable ; c'est l'état le plus voisin de la douleur.

L'impression d'un plaisir faible paraît se concentrer dans l'organe, qui le transmet à l'âme. Mais s'il est à un certain degré de vivacité, il est accompagné d'une émotion qui se répand dans tout le corps. Cette émotion est un fait que notre expérience ne permet pas de révoquer en doute.

La douleur peut également augmenter ou diminuer : en augmentant, elle tend à la destruction totale de l'animal ; mais en diminuant, elle ne tend pas, comme le plaisir, à la privation de tout sentiment ; le moment, qui la termine, est au contraire toujours agréable.

Il n'y a d'état indifférent que par comparai-

son.

Parmi ces différents degrés, il n'est pas possible de trouver un état indifférent : à la première sensation, quelque faible qu'elle soit, la statue est nécessairement bien ou mal. Mais lorsqu'elle aura ressenti successivement les plus vives douleurs et les plus grands plaisirs, elle jugera indifférentes, ou cessera de regarder comme agréables ou désagréables, les sensations plus faibles, qu'elle aura comparées avec les plus fortes.

Nous pouvons donc supposer qu'il y a pour elle des manières d'être agréables et désagréables dans différents degrés, et des manières d'être qu'elle regarde comme indifférentes. 
Origine du besoin.

Toutes les fois qu'elle est mal ou moins bien, elle se rappelle ses sensations passées, elle les compare avec ce qu'elle est, et elle sent qu'il lui est important de redevenir ce qu'elle a été. De là naît le besoin ou la connaissance qu'elle a d'un bien, dont elle juge que la jouissance lui est nécessaire.

Elle ne se connaît donc des besoins, que parce qu'elle compare la peine qu'elle souffre avec les plaisirs dont elle a joui. Enlevez-lui le souvenir de ces plaisirs, elle sera mal, sans soupçonner qu'elle ait aucun besoin : car pour sentir le besoin d'une chose, il faut en avoir quelque connaissance. Or, dans la supposition que nous venons de faire, elle ne connaît d'autre état que celui où elle se trouve. Mais lorsqu'elle s'en rappelle un plus heureux, sa situation présente lui en fait aussitôt sentir le besoin. C'est ainsi que le plaisir et la douleur détermineront toujours l'action de ses facultés.

\section{Comment il détermine les opérations de}

l’âme.

Son besoin peut être occasionné par une véritable douleur, par une sensation désagréable, par une sensation moins agréable que quelques-unes de celles qui ont précédé ; enfin par un état languissant, où elle est réduite à une de ses manières d'être, qu'elle s'est accoutumée à trouver indifférentes.

Si son besoin est causé par une odeur, qui lui fasse une douleur vive, il entraîne à lui presque toute la capacité de sentir ; et il ne laisse de force à la mémoire que pour rappeler à la statue, qu'elle n'a pas toujours été aussi mal. Alors elle est incapable de comparer les différentes manières d'être, par où elle a passé ; elle est incapable de juger qu'elle est la plus agréable. Tout ce qui l'intéresse, c'est de sortir de cet état, pour jouir d'un autre, quel qu'il soit ; et si elle connaissait un moyen qui pût la dérober à sa souffrance, elle appliquerait toutes ses facultés à le mettre en usage. C'est ainsi que dans les grandes maladies, nous cessons de désirer les plaisirs que nous recherchions avec ardeur, et nous ne songeons plus qu’à recouvrer la santé. 
Si c'est une sensation moins agréable qui produise le besoin, il faut distinguer deux cas : ou les plaisirs auxquels la statue la compare ont été vifs, et accompagnés des plus grandes émotions ; ou ils ont été moins vifs, et ne l'ont presque pas émue.

Dans le premier cas, le bonheur passé se réveille avec d'autant plus de force, qu'il diffère davantage de la sensation actuelle. L'émotion qui l'a accompagné, se reproduit en partie, et déterminant vers lui presque toute la capacité de sentir, elle ne permet pas de remarquer les sentiments agréables qui l'ont suivi ou précédé. La statue n'étant donc point distraite, compare mieux ce bonheur avec l'état où elle est ; elle juge mieux combien il en est différent; et s'appliquant à se le peindre de la manière la plus vive, sa privation cause un besoin plus grand, et sa possession devient un bien plus nécessaire.

Dans le second cas, au contraire, il se retrace avec moins de vivacité : d'autres plaisirs partagent l'attention : l'avantage qu'il offre est moins senti : il ne reproduit point, ou que peu d'émotion. La statue n’est donc pas autant intéressée à son retour, et elle n’y applique pas autant ses facultés.

Enfin, si le besoin a pour cause une de ces sensations, qu'elle s'est accoutumée à juger indifférentes, elle vit d'abord sans ressentir ni peine ni plaisir. Mais cet état, comparé aux situations heureuses où elle s'est trouvée, lui devient bientôt désagréable, et la peine qu'elle souffre, est ce que nous appelions ennui. Cependant l'ennui dure, il augmente, il est insupportable, et il détermine avec force toutes les facultés vers le bonheur dont elle sent la perte.

Cet ennui peut être aussi accablant que la douleur : auquel cas, elle n'a d'autre intérêt que de s'y soustraire ; et elle se porte sans choix à toutes les manières d'être, qui sont propres à le dissiper. Mais si nous diminuons le poids de l'ennui, son état sera moins malheureux, il lui importera moins d'en sortir, elle pourra porter son attention à tous les sentiments agréables, dont elle conserve quelque souvenir ; et c'est le plaisir, dont elle se retracera l'idée la plus vive, qui entraînera à lui toutes les facultés. 
Activité qu’il donne à la mémoire.

Il y a donc deux principes qui déterminent le degré d'action de ses facultés : d'un côté, c'est la vivacité d'un bien qu'elle n’a plus ; de l'autre, c'est le peu de plaisir de la sensation actuelle, ou la peine qui l'accompagne.

Lorsque ces deux principes se réunissent, elle fait plus d'effort pour se rappeler ce qu'elle a cessé d'être ; et elle en sent moins ce qu'elle est. Car sa capacité de sentir ayant nécessairement des bornes, la mémoire n'en peut attirer une partie, qu'il n'en reste moins à l'odorat. Si même l'action de cette faculté est assez forte, pour s'emparer de toute la capacité de sentir ; la statue ne remarquera plus l'impression, qui se fait sur son organe, et elle se représentera si vivement ce qu'elle a été, qu'il lui semblera qu'elle l'est encore ${ }^{5}$.

\section{Cette activité cesse avec le besoin.}

Mais si son état présent est le plus heureux qu'elle connaisse, alors le plaisir l'intéresse à en jouir par préférence. Il n’y a plus de cause qui puisse déterminer la mémoire à agir avec assez de vivacité, pour usurper sur l'odorat jusqu'à en éteindre le sentiment. Le plaisir au contraire fixe au moins la plus grande partie de l'attention ou de la capacité de sentir à la sensation actuelle ; et si la statue se rappelle encore ce qu'elle a été, c'est que la comparaison qu'elle en fait avec ce qu'elle est, lui fait mieux goûter son bonheur.

\section{Différence de la mémoire et de l'imagination.}

Voilà donc deux effets de la mémoire : l'un est une sensation qui se retrace aussi vivement, que si elle se faisait sur l'organe même ; l'autre est une sensation, dont il ne reste qu'un souvenir léger.

Notre expérience en est la preuve ; car il n’y a peut-être personne qui ne se soit quelquefois rappelé des plaisirs dont il a joui, avec la même vivacité que s'il en jouissait encore : ou du moins avec assez de vivacité pour ne donner aucune attention à l'état quelquefois affligeant où il se trouve. 
Ainsi il y a dans l'action de cette faculté deux degrés, que nous pouvons fixer : le plus faible est celui, où elle fait à peine jouir du passé ; le plus vif est celui, où elle en fait jouir comme s’il était présent.

Or, elle conserve le nom de mémoire, lorsqu'elle ne rappelle les choses que comme passées ; et elle prend le nom d'imagination, lorsqu'elle les retrace avec tant de force, qu'elles paraissent présentes. L’imagination a donc lieu dans notre statue, aussi bien que la mémoire ; et ces deux facultés ne différent que du plus au moins. La mémoire est le commencement d'une imagination qui n'a encore que peu de force ; l'imagination est la mémoire même, parvenue à toute la vivacité dont elle est susceptible.

Comme nous avons distingué deux attentions, qui se font dans la statue, l'une par l'odorat, l'autre par la mémoire ; nous en pouvons actuellement remarquer une troisième, qu'elle donne par l'imagination, et dont le caractère est d'arrêter les impressions des sens, pour y substituer un sentiment indépendant de l'action des objets extérieurs ${ }^{6}$.

\section{Cette différence échappe à la statue.}

Cependant lorsque la statue imagine une sensation qu'elle n'a plus, et qu'elle se la représente aussi vivement, que si elle l'avait encore ; elle ne sait pas qu'il y a en elle une cause qui produit le même effet qu'un corps odoriférant qui agirait sur son organe. Elle ne peut donc pas mettre, comme nous, de la différence entre imaginer et avoir une sensation.

Mille faits prouvent le pouvoir de l'imagination sur les sens. Un homme fort occupé d'une pensée ne voit point les objets qui sont sous ses yeux, il n'entend pas le bruit qui frappe ses oreilles. Tout le monde sait ce qu'on raconte d'Archimède. Que l'imagination s'applique avec encore plus de force à un objet, on sera piqué, brûlé, sans en ressentir de la douleur ; et l'âme paraîtra se dérober à toutes les impressions des sens. Pour comprendre la possibilité de ces phénomènes, il suffit de considérer que notre capacité de sentir étant bornée, nous serons absolument insensibles aux impressions des sens, toutes les fois que notre imagination l'appliquera toute entière à un objet. 
Son imagination plus active que la nôtre.

Mais on a lieu de présumer que son imagination aura plus d'activité que la nôtre. Sa capacité de sentir est toute entière à une seule espèce de sensation, toute la force de ses facultés s'applique uniquement à des odeurs, rien ne la peut distraire. Pour nous, nous sommes partagés entre une multitude de sensations et d'idées, dont nous sommes sans cesse assaillis ; et ne conservant à notre imagination qu'une partie de nos forces, nous imaginons faiblement. D'ailleurs nos sens toujours en garde contre notre imagination, nous avertissent sans cesse de l'absence des objets que nous voulons imaginer : au contraire tout laisse un libre cours à l'imagination de notre statue. Elle se retrace donc sans défiance une odeur dont elle a joui, et elle en jouit en effet, comme si son organe en était affecté. Enfin la facilité d'écarter de nous les objets qui nous offensent, et de rechercher ceux dont la jouissance nous est chère, contribue encore à rendre notre imagination paresseuse. Mais puisque notre statue ne peut se soustraire à un sentiment désagréable, qu'en imaginant vivement une manière d'être qui lui plaît, son imagination en est plus exercée, et elle doit produire des effets pour lesquels la nôtre est tout-à-fait impuissante ${ }^{7}$.

Cas unique où elle peut être sans action.

Cependant il y a une circonstance, où son action est absolument suspendue, et même encore celle de la mémoire. C'est lorsqu'une sensation est assez vive pour remplir entièrement la capacité de sentir. Alors la statue est toute passive. Le plaisir est pour elle une espèce d'ivresse, où elle en jouit à peine ; et la douleur un accablement, où elle ne souffre presque pas.

Comment elle rentre en action.

Quelque surprenants que soient ces effets de l'imagination, il suffit, pour n'en point douter, de réfléchir sur ce qui nous arrive en songe. Alors nous voyons, nous entendons, nous touchons des corps qui n'agissent point sur nos sens ; et il y a tout lieu de croire que l'imagination n'a tant de force, que parce que nous ne sommes point distraits par la multitude des idées et des sensations qui nous occupent dans la veille. 
Mais que la sensation perde quelques degrés de vivacité, aussitôt les facultés de l'âme rentrent en action, et le besoin redevient la cause qui les détermine.

Elle donne un nouvel ordre aux idées.

Les modifications qui doivent plaire davantage à la statue, ne sont pas toujours les dernières qu'elle a reçues. Elles peuvent se trouver au commencement ou au milieu de la chaîne de ses connaissances, comme à la fin. L'imagination est donc souvent obligée de passer rapidement par-dessus les idées intermédiaires. Elle rapproche les plus éloignées, change l'ordre qu'elles avaient dans la mémoire, et en forme une chaîne toute nouvelle.

La liaison des idées ne suit donc pas le même ordre dans ces facultés. Plus celui qu'elle tient de l'imagination, deviendra familier, moins elle conservera celui que la mémoire lui a donné. Par là, les idées se lient de mille manières différentes; et souvent la statue se souviendra moins de l'ordre dans lequel elle a éprouvé ses sensations, que de celui dans lequel elle les a imaginées.

Les idées ne se lient différemment que parce qu'il s'en fait de nouvelles comparaisons.

Mais toutes ces chaînes ne se forment que par les comparaisons qui ont été faites de chaque anneau avec celui qui le précède, et avec celui qui le suit, et par les jugements qui ont été portés de leurs rapports. Ce lien devient plus fort à proportion que l'exercice des facultés fortifie les habitudes de se souvenir et d'imaginer ; et c'est de là qu'on tire l'avantage surprenant de reconnaître les sensations qu'on a déjà eues.

C'est à cette liaison que la statue reconnaît les manières d'être, qu'elle a eues.

En effet, si nous faisons sentir à notre statue une odeur qui lui est familière ; voilà une manière d'être qu'elle a comparée, dont elle a jugé, et qu'elle a liée à quelques-unes des parties de la chaîne que sa mémoire est dans l'habitude de parcourir. C'est pourquoi elle juge que l'état où elle se trouve, est le même que celui où elle s'est déjà trouvée. Mais une odeur qu'elle n’a point encore sentie, n’est pas dans le même cas ; elle doit donc lui paraître toute nouvelle. 
Elle ne saurait se rendre raison de ce phénomène.

Il est inutile de remarquer que, lorsqu'elle reconnaît une manière d'être, c'est sans être capable de s'en rendre raison. La cause d'un pareil phénomène est si difficile à démêler, qu'elle échappe à tous les hommes, qui ne savent pas observer et analyser ce qui se passe en eux-mêmes.

Comment les idées se conservent et se renouvellent dans la mémoire.

Mais lorsque la statue est longtemps sans penser à une manière d'être, que devient pendant tout cet intervalle l'idée qu'elle en a acquise ? D’où sort cette idée, lorsqu'ensuite elle se retrace à la mémoire ? S'est-elle conservée dans l'âme ou dans le corps ? Ni dans l'un ni dans l'autre.

Ce n'est pas dans l'âme, puisqu'il suffit d'un dérangement dans le cerveau, pour ôter le pouvoir de la rappeler.

Ce n'est pas dans le corps. Il n’y a que la cause physique qui pourrait s'y conserver ; et pour cela, il faudrait supposer que le cerveau restât absolument dans l'état où il a été mis par la sensation que la statue se rappelle. Mais comment accorder cette supposition avec le mouvement continuel des esprits ? Comment l'accorder, surtout quand on considère la multitude d'idées dont la mémoire s'enrichit? On peut expliquer ce phénomène d'une manière bien plus simple.

J'ai une sensation, lorsqu'il se fait dans un de mes organes, un mouvement qui se transmet jusqu'au cerveau. Si le même mouvement commence au cerveau, et s'étend jusqu'à l'organe, je crois avoir une sensation que je n'ai pas : c'est une illusion. Mais si ce mouvement commence et se termine au cerveau, je me souviens de la sensation que j'ai eue.

Quand une idée se retrace à la statue, ce n'est donc pas qu'elle se soit conservée dans le corps ou dans l'âme : c'est que le mouvement, qui en est la cause physique et occasionnelle, se reproduit dans le cerveau $^{8}$. Mais ce n’est pas ici le lieu de hasarder des conjectures sur le

8 Voyez la Logique, part. 1, chap. 9. 
mécanisme de la mémoire. Nous conservons le souvenir de nos sensations, nous nous les rappelons, après avoir été longtemps sans y penser : il suffit pour cela qu'elles aient fait sur nous une vive impression, ou que nous les ayons éprouvées à plusieurs reprises. Ces faits m'autorisent à supposer que notre statue étant organisée comme nous, est, comme nous, capable de mémoire.

Énumération des habitudes contractées par la

statue.

Concluons qu'elle a contracté plusieurs habitudes : une habitude de donner son attention, une autre de se ressouvenir, une troisième de comparer, une quatrième de juger, une cinquième d'imaginer, et une dernière de reconnaître.

\section{Comment ses habitudes s'entretiendront.}

Les mêmes causes qui ont produit les habitudes, sont seules capables de les entretenir. Je veux dire que les habitudes se perdront, si elles ne sont pas renouvelées par des actes réitérés de temps à autre. Alors notre statue ne se rappellera ni les comparaisons qu'elle a faites d'une manière d'être, ni les jugements qu'elle en a portés, et elle l'éprouvera pour la troisième ou quatrième fois, sans être capable de la reconnaître.

\section{Se fortifieront.}

Mais nous pouvons nous-mêmes contribuer à entretenir l'exercice de sa mémoire et de toutes ses facultés. Il suffit de l'intéresser par les différents degrés de plaisir ou de peine à conserver ses manières d'être, ou à s'y soustraire. L'art avec lequel nous disposerons de ses sensations, pourra donc donner occasion de fortifier et d'étendre de plus en plus ses habitudes. Il y a même lieu de conjecturer qu'elle démêlera dans une succession d'odeurs des différences, qui nous échappent. Obligée d'appliquer toutes ses facultés à une seule espèce de sensation, pourrait-elle ne pas apporter à cette étude plus de discernement que nous? 
Quelles sont les bornes de son discernement.

Cependant les rapports que ses jugements peuvent découvrir, sont en fort petit nombre. Elle connaît seulement qu'une manière d'être, est la même que celle qu'elle a déjà eue, ou qu'elle en est différente ; que l'une est agréable, l'autre désagréable, qu'elles le sont plus ou moins.

Mais démêlera-t-elle plusieurs odeurs, qui se font sentir ensemble ? C'est un discernement que nous n'acquérons nous-mêmes que par un grand exercice : encore est-il renfermé dans des bornes bien étroites : car il n'est personne qui puisse reconnaître à l'odorat tout ce qui compose un sachet. Or, tout mélange d'odeurs me paraît devoir être un sachet pour notre statue.

C'est la connaissance des corps odoriférants, comme nous verrons ailleurs, qui nous a appris à reconnaître deux odeurs dans une troisième. Après avoir senti tour-à-tour une rose et une jonquille, nous les avons senties ensemble, et par là nous avons appris que la sensation que ces fleurs réunies font sur nous, est composée de deux autres. Qu'on multiplie les odeurs, nous ne distinguerons que celles qui dominent ; et même nous n'en ferons pas le discernement, si le mélange est fait avec assez d'art, pour qu'aucune ne prévale. En pareil cas elles paraissent se confondre à-peu-près, comme des couleurs broyées ensemble ; elles se réunissent, et se mêlent si bien, qu'aucune d'elles ne reste ce qu'elle était ; et de plusieurs il n’en résulte qu'une seule.

Si notre statue sent deux odeurs au premier moment de son existence, elle ne jugera donc pas qu'elle est tout-à-la-fois de deux manières. Mais supposons qu'ayant appris à les connaître séparément, elle les sente ensemble, les reconnaîtra-t-elle ? Cela ne me paraît pas vraisemblable. Car, ignorant qu'elles lui viennent de deux corps différents, rien ne peut lui faire soupçonner que la sensation qu'elle éprouve, est formée de deux autres. En effet, si aucune ne domine, elles se confondraient même à notre égard ; et s'il en est une qui soit plus faible, elle ne fera qu'altérer la plus forte, et elles paraîtront ensemble comme une simple manière d'être. Pour nous en convaincre, nous n'aurions qu'à sentir des odeurs, que nous ne nous serions pas fait une habitude de rapporter à des corps différents : je suis persuadé que nous 
n'oserions assurer si elles ne sont qu'une, ou si elles sont plusieurs. Voilà précisément le cas de notre statue.

Elle n'acquiert donc du discernement, que par l'attention qu'elle donne en même temps à une manière d'être qu'elle éprouve, et à une autre qu'elle a éprouvé. Ainsi ses jugements ne s'exercent point sur deux odeurs senties à-la-fois ; ils n'ont pour objet, que des sensations qui se succèdent. 


\section{Chapitre III}

\section{Des désirs, des passions, de l'amour, de la haine, de l'espérance, de la crainte et de la volonté dans un homme borné au sens de l'odorat}

Le désir n'est que l'action des facultés.

Nous venons de faire voir en quoi consistent les différentes sortes de besoins, et comment ils sont la cause des degrés de vivacité, avec lesquels les facultés de l'âme s'appliquent à un bien, dont la jouissance devient nécessaire. Or, le désir n'est que l'action même de ces facultés, lorsqu'elles se dirigent sur la chose dont nous sentons le besoin ${ }^{9}$.

\section{Ce qui en fait la faiblesse ou la force.}

Tout désir suppose donc que la statue a l'idée de quelque chose de mieux, que ce qu'elle est dans le moment ; et qu'elle juge de la différence de deux états qui se succèdent. S'ils diffèrent peu, elle souffre moins, par la privation de la manière d'être qu'elle désire ; et j'appelle malaise, ou léger mécontentement, le sentiment qu'elle éprouve : alors l'action de ses facultés, ses désirs sont plus faibles. Elle souffre au contraire davantage, si la différence est considérable ; et j'appelle inquiétude, ou même tourment, l'impression qu'elle ressent: alors l'action de ses facultés, ses désirs sont plus vifs. La mesure du désir est donc la différence aperçue entre ces deux états ; et il suffit de se rappeler comment l'action des facultés peut acquérir, ou perdre de la vivacité, pour connaître tous les degrés, dont les désirs sont susceptibles.

9 Logique. Leçons préliminaires du cours d’études 
Une passion est un désir dominant.

Ils n'ont, par exemple, jamais plus de violence, que lorsque les facultés de la statue se portent à un bien, dont la privation produit une inquiétude d'autant plus grande, qu'il diffère davantage de la situation présente. En pareil cas, rien ne la peut distraire de cet objet : elle se le rappelle, elle l'imagine ; toutes ses facultés s'en occupent uniquement. Plus par conséquent elle le désire, plus elle s’accoutume à le désirer. En un mot, elle a pour lui ce qu'on nomme passion; c'est-à-dire, un désir qui ne permet pas d'en avoir d'autres, ou qui du moins est le plus dominant.

Comment une passion succède à une autre.

Cette passion subsiste, tant que le bien qui en est l'objet, continue de paraître le plus agréable, et que sa privation est accompagnée des mêmes inquiétudes. Mais elle est remplacée par une autre, si la statue a occasion de s'accoutumer à un nouveau bien auquel elle doit donner la préférence.

Ce que c'est que l'amour et la haine.

Dès qu'il y a en elle jouissance, souffrance, besoin, désir, passion, il y a aussi amour et haine. Car elle aime une odeur agréable, dont elle jouit, ou qu'elle désire. Elle hait une odeur désagréable, qui la fait souffrir : enfin, elle aime moins une odeur moins agréable qu'elle voudrait changer contre une autre. Pour s'en convaincre, il suffit de considérer qu'aimer est toujours synonyme de jouir, ou de désirer ; et que haïr l'est également de souffrir du malaise, du mécontentement à la présence d'un objet.

L'un et l'autre susceptibles de différents degrés.

Comme il peut y avoir plusieurs degrés dans l'inquiétude, que cause la privation d'un objet aimable, et dans le mécontentement, que donne la vue d'un objet odieux ; il en faut également distinguer dans l'amour et dans la haine. Nous avons même des mots à cet usage : tels sont ceux de goût, penchant, inclination ; d'éloignement, répugnance, dégoût. Quoiqu'on ne puisse pas substituer à ces mots ceux d'amour 
et de haine, les sentiments qu'ils expriment, ne sont néanmoins qu'un commencement de ces passions : ils n'en diffèrent, que parce qu'ils sont dans un degré plus faible.

La statue ne peut aimer qu'elle-même.

Au reste, l'amour, dont notre statue est capable, n'est que l'amour d'elle-même, ou, ce qu'on nomme l'amour-propre. Car dans le vrai elle n'aime qu'elle ; puisque les choses qu'elle aime, ne sont que ses propres manières d'être.

Principes de l'espérance et de la crainte.

L'espérance et la crainte naissent du même principe que l'amour et la haine.

L'habitude, où est notre statue d'éprouver des sensations agréables et désagréables, lui fait juger qu'elle en peut éprouver encore. Si ce jugement se joint à l'amour d'une sensation qui plaît, il produit l'espérance ; et s'il se joint à la haine d'une sensation qui déplaît, il forme la crainte. En effet, espérer, c'est se flatter de la jouissance d'un bien ; craindre, c'est se voir menacé d'un mal. Nous pouvons remarquer que l'espérance et la crainte contribuent à augmenter les désirs. C'est du combat de ces deux sentiments, que naissent les passions les plus vives.

\section{Comment la volonté se forme.}

Le souvenir d'avoir satisfait quelques-uns de ses désirs, fait d'autant plus espérer à notre statue d'en pouvoir satisfaire d'autres ; que ne connaissant pas les obstacles, qui s'y opposent, elle ne voit pas pourquoi ce qu'elle désire, ne serait pas en son pouvoir, comme ce qu'elle a désiré en d'autres occasions. A la vérité, elle ne peut s'en assurer ; mais aussi elle n'a point de preuve du contraire. Si elle se souvient surtout que le même désir, qu'elle forme, a d'autres fois été suivi de la jouissance ; elle se flattera, à proportion que son besoin sera plus grand. Ainsi deux causes contribuent à sa confiance : l'expérience d'avoir satisfait un pareil désir, et l'intérêt, qu'il le soit 
encore ${ }^{10}$. Dès lors elle ne se borne plus à désirer : elle veut ; car on entend par volonté, un désir absolu, et tel, que nous pensons qu'une chose désirée est en notre pouvoir.

Table des matières

10 Il en est de notre statue comme de tous les hommes. Nous nous conduisons d'après l'expérience, et nous nous faisons différentes règles de probabilité, suivant l'intérêt qui nous domine. S’il est grand, le plus léger degré de probabilité nous suffit ordinairement; et lorsque nous sommes assez sages pour ne nous déterminer que sur une probabilité bien fondée, ce n’est souvent que parce que nous avons peu d'intérêt à agir. 


\section{Chapitre IV}

\section{Des idées d'un homme borné au sens de l'odorat}

La statue a les idées de contentement et de mécontentement.

Notre statue ne peut être successivement de plusieurs manières, dont les unes lui plaisent, et les autres lui déplaisent, sans remarquer qu'elle passe tour-à-tour par un état de plaisir, et par un état de peines. Avec les unes, c'est contentement, jouissance ; avec les autres, c'est mécontentement, souffrance. Elle conserve donc dans sa mémoire les idées de contentement et de mécontentement, communes à plusieurs manières d'être : et elle n'a plus qu'à considérer ses sensations sous ces deux rapports, pour en faire deux classes, où elle apprendra à distinguer des nuances, à proportion qu'elle s’y exercera davantage.

Ces idées sont abstraites et générales.

Abstraire, c'est séparer une idée d'une autre, à laquelle elle paraît naturellement unie. Or, en considérant que les idées de contentement et de mécontentement sont communes à plusieurs de ses modifications, elle contracte l'habitude de les séparer de telle modification particulière, dont elle ne l'avait pas d'abord distinguée; elle s'en fait donc des notions abstraites ; et ces notions deviennent générales, parce qu'elles sont communes à plusieurs de ces manières d'être.

Une odeur n'est pour la statue qu'une idée particulière.

Mais lorsqu'elle sentira successivement plusieurs fleurs de même espèce, elle éprouvera toujours une même manière d'être, et elle n'aura à ce sujet qu'une idée particulière. L'odeur de violette, par exemple, ne saurait être pour elle une idée abstraite, commune à plusieurs fleurs ; puisqu'elle ne sait pas qu'il existe des violettes. Ce n'est 
donc que l'idée particulière d'une manière d'être qui lui est propre. Par conséquent, toutes ses abstractions se bornent à des modifications plus ou moins agréables, et à d’autres plus ou moins désagréables.

\section{Comment le plaisir, en général, devient l’objet} de sa volonté.

Lorsqu'elle n'avait que des idées particulières, elle ne pouvait désirer que telle ou telle manière d'être. Mais aussitôt qu'elle a des notions abstraites, ses désirs, son amour, sa haine, son espérance, sa crainte, sa volonté, peuvent avoir pour objet le plaisir ou la peine en général.

Cependant cet amour du bien en général n’a lieu, que lorsque dans le nombre d'idées, que la mémoire lui retrace confusément, elle ne distingue pas encore ce qui doit lui plaire davantage ; mais dès qu'elle croit l'apercevoir, alors tous ses désirs se tournent vers une manière d'être en particulier.

Elle a des idées de nombre.

Puisqu'elle distingue les états par où elle passe, elle a quelque idée de nombre : elle a celle de l'unité, toutes les fois qu'elle éprouve une sensation, ou qu'elle s'en souvient; et elle a les idées de deux et de trois, toutes les fois que sa mémoire lui rappelle deux ou trois manières d'être distinctes : car elle prend alors connaissance d'elle-même, comme étant une odeur, ou, comme en ayant été deux ou trois successivement.

Elle ne les doit qu’à sa mémoire.

Elle ne peut pas distinguer deux odeurs, qu'elle sent à-la-fois. L'odorat par lui-même ne saurait donc lui donner que l'idée de l'unité, et elle ne peut tenir les idées des nombres que de la mémoire. 
Jusqu'où elle peut les étendre.

Mais elle n'étendra pas bien loin ses connaissances à ce sujet. Ainsi qu'un enfant, qui n’a pas appris à compter, elle ne pourra pas déterminer le nombre de ses idées, lorsque la succession en aura été un peu considérable.

Il me semble que, pour découvrir la plus grande quantité, qu'elle est capable de connaître distinctement, il suffit de considérer jusqu'où nous pourrions nous-mêmes compter avec le signe un. Quand les collections formées par la répétition de ce mot, ne pourront pas être saisies tout-à-la-fois d'une manière distincte; nous serons en droit de conclure, que les idées précises des nombres qu'elles renferment, ne peuvent pas s’acquérir par la seule mémoire.

Or, en disant un et un, j'ai l'idée de deux ; et en disant un, un et un, j'ai l'idée de trois. Mais si je n'avais, pour exprimer dix, quinze, vingt, que la répétition de ce signe, je n’en pourrais jamais déterminer les idées : car je ne saurais m’assurer par la mémoire, d'avoir répété un autant de fois, que chacun de ces nombres le demande. Il me paraît même que je ne saurais par ce moyen me faire l'idée de quatre ; et que j'ai besoin de quelque artifice, pour être sûr de n'avoir répété ni trop ni trop peu le signe de l'unité. Je dirai, par exemple, un, un, et puis un, un : mais cela seul prouve que la mémoire ne saisit pas distinctement quatre unités à-la-fois. Elle ne présente donc au-delà de trois qu'une multitude indéfinie. Ceux qui croiront qu'elle peut seule étendre plus loin nos idées, substitueront un autre nombre à celui de trois. Il suffit, pour les raisonnements que j'ai à faire, de convenir qu'il y en a un audelà duquel la mémoire ne laisse plus apercevoir qu'une multitude tout-à-fait vague. C'est l'art des signes qui nous a appris à porter la lumière plus loin. Mais quelque considérables que soient les nombres que nous pouvons démêler, il reste toujours une multitude, qu'il n'est pas possible de déterminer, qu'on appelle par cette raison l'infini, et qu'on eût bien mieux nommé l'indéfini. Ce seul changement de nom eût prévenu des erreurs ${ }^{11}$.

11 Principalement l'erreur de croire que nous avons une idée positive de l'infini ; d'où quantité de mauvais raisonnements de la part des métaphysiciens, et quelquefois même de celle des géomètres. 
Nous pouvons donc conclure que notre statue n'embrassera distinctement que jusqu'à trois de ses manières d'être. Au-delà elle en verra une multitude, qui sera pour elle ce qu'est la notion prétendue de l'infini pour nous. Elle sera même bien plus excusable de s'y méprendre : car elle est incapable des réflexions, qui pourraient la tirer d'erreur. Elle apercevra donc l'infini dans cette multitude, comme s'il y était en effet.

Enfin, nous remarquerons que son idée de l'unité est abstraite : car elle sent toutes ses manières d'être sous ce rapport général, que chacune est distinguée de toute autre.

Elle connaît deux sortes de vérités; des

vérités particulières, des vérités générales.

Comme elle a des idées particulières et des idées générales, elle connaît deux sortes de vérités.

Les odeurs de chaque espèce de fleurs ne sont pour elle que des idées particulières. Il en sera donc de même de toutes les vérités qu'elle aperçoit, lorsqu'elle distingue une odeur d'une autre.

Mais elle a les notions abstraites de manières d'être agréables, et de manières d'être désagréables. Elle connaîtra donc à ce sujet des vérités générales : elle saura qu’en général ses modifications diffèrent les unes des autres, et qu'elles lui plaisent ou déplaisent plus ou moins.

Mais ces connaissances générales supposent en elle des connaissances particulières, puisque les idées particulières ont précédé les notions abstraites.

Elle a quelque idée du possible.

Comme elle est dans l'habitude d'être, de cesser d'être, et de redevenir la même odeur ; elle jugera, lorsqu'elle ne l'est pas, qu'elle pourra l'être ; lorsqu'elle l'est, qu'elle pourra ne l'être plus. Elle aura donc occasion de considérer ses manières d'être, comme pouvant exister, ou ne pas exister. Mais cette notion du possible ne portera point avec elle la connaissance des causes, qui peuvent produire un effet : elle en supposera au contraire l'ignorance, et elle ne sera fondée que sur un jugement d'habitude. Lorsque la statue pense qu'elle peut, 
par exemple, cesser d'être odeur de rose, et redevenir odeur de violette, elle ignore qu'un être extérieur dispose uniquement de ses sensations. Pour qu'elle se trompe dans son jugement, il suffit que nous nous proposons de lui faire sentir continuellement la même odeur. Il est vrai que son imagination y peut quelquefois suppléer: mais ce n'est que dans les occasions, où les désirs sont violents ; encore même n’y réussit-elle pas toujours.

\section{Peut-être encore de l'impossible.}

Peut-être pourrait-elle, d'après ses jugements d'habitude, se faire aussi quelque idée de l'impossible. Accoutumée à perdre une manière d'être, aussitôt qu'elle en acquiert une nouvelle, il est impossible, suivant sa manière de concevoir, qu'elle en ait deux à-la-fois. Le seul cas, où elle croirait le contraire, ce serait celui où son imagination agirait avec assez de force, pour lui retracer deux sensations avec la même vivacité que si elle les éprouvait réellement. Mais cela ne peut guère arriver. Il est naturel que son imagination se conforme aux habitudes qu'elle s'est faite. Ainsi n'ayant éprouvé ses manières d'être que l'une après l'autre, elle ne les imaginera que dans cet ordre. D'ailleurs, sa mémoire n'aura pas vraisemblablement assez de force, pour lui rendre présentes deux sensations qu'elle a eues, et qu'elle n'a plus.

Mais ce qui me paraît plus probable, c'est que si l'habitude où elle est de juger, que ce qui lui est arrivé, peut lui arriver encore, renferme l'idée du possible ; il est bien difficile qu'elle ait occasion de former des jugements où nous puissions retrouver l'idée que nous avons de l'impossible. Il faudrait pour cela qu'elle s'occupât de ce qu'elle n'a point encore éprouvé ; mais il est bien plus naturel qu'elle soit toute entière à ce qu'elle éprouve.

Elle a l’idée d’une durée passée.

Du discernement qui se fait en elle des odeurs, naît une idée de succession : car elle ne peut sentir qu'elle cesse d'être ce qu'elle était, sans se représenter dans ce changement une durée de deux instants. 
Comme elle n'embrasse d'une manière distincte que jusqu'à trois odeurs, elle ne démêlera aussi que trois instants dans sa durée. Audelà elle ne verra qu'une succession indéfinie.

Si l'on suppose que la mémoire peut lui rappeler distinctement jusqu'à quatre, cinq, six manières d'être, elle distinguera en conséquence quatre, cinq, six instants dans sa durée. Chacun peut faire à ce sujet les hypothèses qu'il jugera à propos, et les substituer à celles que j'ai cru devoir préférer.

D’une durée à venir.

Le passage d'une odeur à une autre ne donne à notre statue que l'idée du passé. Pour en avoir une de l'avenir, il faut qu'elle ait eu à plusieurs reprises la même suite de sensations ; et qu'elle se soit fait une habitude de juger, qu'après une modification une autre doit suivre.

Prenons pour exemple cette suite, jonquille, rose, violette. Dès que ces odeurs sont constamment liées dans cet ordre, une d'elles ne peut affecter son organe, qu'aussitôt la mémoire ne lui rappelle les autres dans le rapport où elles sont à l'odeur sentie. Ainsi qu'à l'occasion de l'odeur de violette, les deux autres se retraceront comme ayant précédé, et qu'elle se représentera une durée passée ; de même à l'occasion de l'odeur de jonquille, celles de rose et de violette se retraceront comme devant suivre, et elle se représentera une durée à venir.

\section{D’une durée indéfinie.}

Les odeurs de jonquille, de rose et de violette peuvent donc marquer les trois instants qu'elle aperçoit d'une manière distincte. Par la même raison, les odeurs qui ont précédé, et celles qui sont dans l'habitude de suivre, marqueront les instants qu'elle aperçoit confusément dans le passé et dans l'avenir. Ainsi, lorsqu'elle sentira une rose, sa mémoire lui rappellera distinctement l'odeur de jonquille et celle de violette; et elle lui représentera une durée indéfinie, qui a précédé l'instant où elle sentait la jonquille, et une durée indéfinie, qui doit suivre celui où elle sentira la violette. 
Cette durée est pour elle une éternité.

Apercevant cette durée comme indéfinie, elle n’y peut démêler ni commencement ni fin: elle n'y peut même soupçonner ni l'un ni l'autre. C'est donc à son égard une éternité absolue ; et elle se sent, comme si elle eût toujours été, et qu'elle ne dût jamais cesser d'être.

En effet, ce n'est point la réflexion sur la succession de nos idées, qui nous apprend que nous avons commencé, et que nous finirons : c'est l'attention que nous donnons aux êtres de notre espèce, que nous voyons naître et périr. Un homme qui ne connaîtrait que sa propre existence, n’aurait aucune idée de la mort.

Il y a en elle deux successions.

L’idée de la durée d'abord produite par la succession des impressions qui se font sur l'organe, se conserve, ou se reproduit par la succession des sensations que la mémoire rappelle. Ainsi, lors même que les corps odoriférants n'agissent plus sur notre statue, elle continue de se représenter le présent, le passé et l'avenir. Le présent, par l'état où elle se trouve ; le passé, par le souvenir de ce qu'elle a été ; l'avenir, parce qu'elle juge qu'ayant eu à plusieurs reprises les mêmes sensations, elle peut les avoir encore.

Il y a donc en elle deux successions; celle des impressions faites sur l’organe, et celle des sensations qui se retracent à la mémoire.

L'une de ces successions mesure les moments de l'autre.

Plusieurs impressions peuvent se succéder dans l'organe, pendant que le souvenir d'une même sensation est présent à la mémoire ; et plusieurs sensations peuvent se retracer successivement à la mémoire, pendant qu'une même impression se fait éprouver à l'organe. Dans le premier cas, la suite des impressions qui se font à l'odorat, mesure la durée du souvenir d'une sensation : dans le second, la suite des sensations qui s'offrent à la mémoire, mesure la durée de l’impression que l’odorat reçoit. 
$\mathrm{Si}$, par exemple, lorsque la statue sent une rose, elle se rappelle des odeurs de tubéreuse, de jonquille et de violette ; c'est à la succession qui se passe dans sa mémoire, qu'elle juge de la durée de sa sensation : et si, lorsqu'elle se retrace l'odeur de rose, je lui présente rapidement une suite de corps odoriférants; c'est à la succession qui se passe dans l'organe, qu'elle juge de la durée du souvenir de cette sensation. Elle aperçoit donc qu'il n'est aucune de ses modifications, qui ne puisse durer. La durée devient un rapport, sous lequel elle les considère toutes en général, et elle s'en fait une notion abstraite.

$\mathrm{Si}$, dans le temps qu'elle sent une rose, elle se rappelle successivement les odeurs de violette, de jasmin et de lavande; elle s'apercevra comme une odeur de rose, qui dure trois instants : et si elle se retrace une suite de vingt odeurs, elle s'apercevra comme étant odeur de rose depuis un temps indéfini ; elle ne jugera plus qu'elle ait commencé de l'être, elle croira l’être de toute éternité.

\section{L’idée de durée n’est pas absolue.}

Il n'y a donc qu'une succession d'odeurs transmises par l'organe, ou renouvelées par la mémoire, qui puisse lui donner quelque idée de durée. Elle n'aurait jamais connu qu'un instant, si le premier corps odoriférant eût agi sur elle d'une manière uniforme, pendant une heure, un jour ou davantage ; ou, si son action eût varié par des nuances si insensibles, qu'elle n'eût pu les remarquer.

Il en sera de même, si ayant acquis l'idée de durée, elle conserve une sensation, sans faire usage de sa mémoire, sans se rappeler successivement quelques-unes des manières d'être, par où elle a passé. Car à quoi y distinguerait-elle des instants ? Et si elle n'en distingue pas, comment en apercevra-t-elle la durée ?

L’idée de la durée n'est donc point absolue, et lorsque nous disons que le temps coule rapidement, ou lentement, cela ne signifie autre chose, sinon que les révolutions qui servent à le mesurer, se font avec plus de rapidité, ou avec plus de lenteur, que nos idées ne se succèdent. On peut s'en convaincre par une supposition. 
Supposition qui le rend sensible.

Si nous imaginons qu'un monde composé d'autant de parties que le nôtre, ne fût pas plus gros qu'une noisette ; il est hors de doute que les astres s'y lèveraient, et s'y coucheraient des milliers de fois dans une de nos heures ; et qu'organisés, comme nous le sommes, nous n'en pourrions pas suivre les mouvements. Il faudrait donc que les organes des intelligences destinées à l'habiter, fussent proportionnés à des révolutions aussi subites ${ }^{12}$.

Ainsi, pendant que la terre de ce petit monde tournera sur son axe, et autour de son soleil, ses habitants recevront autant d'idées, que nous en avons pendant que notre terre fait de semblables révolutions. Dès lors il est évident que leurs jours et leurs années leur paraîtront aussi longs, que les nôtres nous le paraissent.

En supposant un autre monde auquel le nôtre serait aussi inférieur, qu'il est supérieur à celui que je viens de feindre ; il faudrait donner à ses habitants des organes, dont l'action serait trop lente, pour apercevoir les révolutions de nos astres. Ils seraient, par rapport à notre monde, comme nous par rapport à ce monde gros comme une noisette. Ils n’y sauraient distinguer aucune succession de mouvement.

Demandons enfin aux habitants de ces mondes quelle en est la durée : ceux du plus petit compteront des millions de siècles, et ceux du plus grand ouvrant à peine les yeux, répondront qu'ils ne font que de naître.

La notion de la durée est donc toute relative : chacun n'en juge que par la succession de ses idées ; et vraisemblablement il n’y a pas deux hommes qui, dans un temps donné, comptent un égal nombre d'instants. Car il y a lieu de présumer qu'il n’y en a pas deux, dont la mémoire retrace toujours les idées avec la même rapidité.

Par conséquent, une sensation, qui se conservera uniformément pendant un an, ou mille, si l'on veut, ne sera qu'un instant à l'égard de notre statue ; comme une idée que nous conservons, pendant que les

12 Malebranche fait une pareille supposition, pour prouver que nous ne jugeons de la grandeur des corps que par les rapports qui sont entre eux et nous. $R e-$ cher. de la Vér., liv. 1, chap. 6. 
habitants du petit monde comptent des siècles, est un instant pour nous ${ }^{13}$. C'est donc une erreur de penser que tous les êtres jugent également de la durée, et comptent le même nombre d'instants. La présence d'une idée, qui ne varie point, n'étant qu'un instant à notre égard, c'est une conséquence, qu'un instant de ma durée puisse coexister à plusieurs instants de la durée d'un autre.

13 La supposition de ces mondes fait comprendre que, pour les imaginer plus anciens les uns que les autres, il n'est pas nécessaire d'une éternité successive, dans laquelle ils aient été créés plus tôt ou plus tard ; il suffit de varier les révolutions, et d'y proportionner les organes des habitants.

Cette supposition fait encore connaître qu'un instant de la durée d'un être peut co-exister, et co-existe en effet à plusieurs instants de la durée d'un autre. Nous pouvons donc imaginer des intelligences qui aperçoivent, tout-à-la-fois des idées que nous n'avons que successivement, et arriver en quelque sorte jusqu'à un esprit qui embrasse dans un instant toutes les connaissances que les créatures n’ont que dans une suite de siècles, et qui, par conséquent, n'essuie aucune succession. Il sera comme au centre de tous ces mondes, où l'on juge si différemment de la durée ; et, saisissant d'un coup-d'œil tout ce qui leur arrive, il en verra tout-à-la-fois le passé, le présent et l'avenir.

Par ce moyen nous nous formons, autant qu'il est en notre pouvoir, idée d'un instant indivisible et permanent, auquel les instants des créatures coexistent, et dans lequel ils se succèdent. Je dis autant qu'il est en notre pouvoir ; car ce n'est ici qu'une idée de comparaison. Ni nous, ni toute autre créature, ne pourrons avoir une notion parfaite de l'éternité. Dieu seul la connaît, parce que lui seul en jouit. 
Table des matières

\section{Chapitre V}

Du sommeil et des songes d'un homme borné à l'odorat

\section{Comment l’action des facultés se ralentit.}

Notre statue peut être réduite à n'être que le souvenir d'une odeur ; alors le sentiment de son existence paraît lui échapper. Elle sent moins qu'elle existe, qu'elle ne sent qu'elle a existé ; et à proportion que sa mémoire lui retrace les idées avec moins de vivacité, ce reste de sentiment s'affaiblit encore. Semblable à une lumière qui s'éteint par degrés, il cesse tout-à-fait, lorsque cette faculté tombe dans une entière inaction.

État du sommeil.

Or, notre expérience ne nous permet pas de douter que l'exercice ne doive enfin fatiguer la mémoire et l'imagination de notre statue. Considérons donc ces facultés en repos, et ne les excitons par aucune sensation : cet état sera celui du sommeil.

État de songe.

Si leur repos est tel, qu'elles soient absolument sans action, on ne peut remarquer autre chose, sinon que le sommeil est le plus profond qu'il soit possible. Si au contraire elles continuent encore d'agir, ce ne sera que sur une partie des idées acquises. Plusieurs anneaux de la chaîne seront donc interceptés, et l'ordre des idées, dans le sommeil, ne pourra pas être le même que dans la veille. Le plaisir ne sera plus l'unique cause qui déterminera l'imagination. Cette faculté ne réveillera que les idées sur lesquelles elle conserve quelque pouvoir ; et elle contribuera aussi souvent au malheur de notre statue qu'à son bonheur. 
En quoi il diffère de la veille.

Voilà l'état de songe : il ne diffère de celui de la veille, que parce que les idées n’y conservent pas le même ordre, et que le plaisir n'est pas toujours la loi qui règle l'imagination. Tout songe suppose donc quelques idées interceptées, sur lesquelles les facultés de l'âme ne peuvent plus agir.

La statue n'en saurait faire la différence.

Puisque notre statue ne connaît point de différence entre imaginer vivement, et avoir des sensations ; elle n'en saurait faire entre songer et veiller. Tout ce qu'elle éprouve étant endormie, est donc aussi réel à son égard, que ce qu'elle a éprouvé avant le sommeil. 


\section{Chapitre VI}

\section{Du moi, ou de la personnalité d'un homme borné à l'odorat}

De la personnalité de la statue.

Notre statue étant capable de mémoire, elle n'est point une odeur, qu'elle ne se rappelle d'en avoir été une autre. Voilà sa personnalité : car, si elle pouvait dire moi, elle le dirait dans tous les instants de sa durée ; et à chaque fois son moi embrasserait tous les moments, dont elle conserverait le souvenir.

Elle ne peut pas dire moi au premier moment de son existence.

A la vérité, elle ne le dirait pas à la première odeur. Ce qu'on entend par ce mot, ne me paraît convenir qu'à un être qui remarque que, dans le moment présent, il n'est plus ce qu'il a été. Tant qu’il ne change point, il existe sans aucun retour sur lui-même : mais aussitôt qu'il change, il juge qu'il est le même qui a été auparavant de telle manière, et il dit moi.

Cette observation confirme qu'au premier instant de son existence, la statue ne peut former des désirs : car avant de pouvoir dire, je désire, il faut avoir dit, moi, ou je.

Son moi est tout-à-la-fois la conscience de ce qu'elle est, et le souvenir de ce qu'elle a été.

Les odeurs, dont la statue ne se souvient pas, n'entrent donc point dans l'idée qu'elle a de sa personne. Aussi étrangères à son moi, que les couleurs et les sons, dont elle n'a encore aucune connaissance ; elles sont à son égard, comme si elle ne les avait jamais senties. Son moi n'est que la collection des sensations qu'elle éprouve, et de celles 
que la mémoire lui rappelle ${ }^{14}$. En un mot, c'est tout-à-la-fois et la conscience de ce qu'elle est, et le souvenir de ce qu'elle a été.

14 «Celui qui aime une personne, dit Pascal (c. 24, n. 14), à cause de sa beauté, l'aime-t-il ? Non ; car la petite vérole qui ôtera la beauté, sans tuer la personne fera qu'il ne l'aimera plus. Et si on m'aime pour mon jugement ou pour ma mémoire, m’aime-t-on, moi ? Non ; car je puis perdre ces qualités sans cesser d'être. Où est donc le moi, s'il n'est ni dans le corps, ni dans l'âme ? Et comment aimer le corps et l'âme, sinon pour des qualités qui ne sont point ce qui fait le moi, puisqu'elles sont périssables ? Car aimerait-on la substance de l'âme d'une personne abstraitement, et quelques qualités qui y fussent ? Cela ne se peut, et serait injuste. On n'aime donc jamais la personne, mais seulement les qualités; ou, si on aime la personne, il faut dire que c'est l'assemblage des qualités qui fait la personne. »

Ce n'est pas l'assemblage des qualités qui fait la personne ; car le même homme, jeune ou vieux, beau ou laid, sage ou fou, serait autant de personnes distinctes ; et pour quelques qualités qu'on m’aime, c'est toujours moi qu'on aime ; car les qualités ne sont que moi modifié différemment. Si quelqu'un me marchant sur le pied, me disait : Vous ai-je blessé, vous ? non ; car vous pourriez perdre le pied, sans cesser d'être. Serais-je bien convaincu de n'avoir point été blessé moi-même ? Pourquoi donc penserais-je que, parce que je puis perdre la mémoire et le jugement, on ne m'aime pas, lorsqu'on m'aime pour ces qualités ? Mais elles sont périssables : et qu'importe ? Le moi est-il donc une chose nécessaire de sa nature ? Ne périt-il pas dans les bêtes ? Et son immortalité dans l'homme n'est-elle pas une faveur de Dieu ? Dans le sens de Pascal, Dieu seul pourrait dire, moi. 


\section{Chapitre VII}

\section{Conclusion des chapitres précédents}

Avec un seul sens, l'âme a le germe de toutes ses facultés.

Ayant prouvé que notre statue est capable de donner son attention, de se ressouvenir, de comparer, de juger, de discerner, d'imaginer ; qu'elle a des notions abstraites, des idées de nombre et de durée; qu'elle connaît des vérités générales et particulières; qu'elle forme des désirs, se fait des passions, aime, hait, veut ; qu'elle est capable d'espérance, de crainte et d'étonnement ; et qu'enfin elle contracte des habitudes : nous devons conclure qu'avec un seul sens l'entendement a autant de facultés, qu'avec les cinq réunis. Nous verrons que celles qui paraissaient nous être particulières, ne sont que ces mêmes facultés, qui s'appliquant à un plus grand nombre d'objets, se développent davantage.

La sensation renferme toutes les facultés de l’âme.

Si nous considérons que se ressouvenir, comparer, juger, discerner, imaginer, être étonné, avoir des idées abstraites, en avoir de nombre et de durée, connaître des vérités générales et particulières, ne sont que différentes manières d'être attentif ; qu'avoir des passions, aimer, haïr, espérer, craindre et vouloir, ne sont que différentes manières de désirer ; et qu'enfin être attentif, et désirer, ne sont dans l'origine que sentir : nous conclurons que la sensation enveloppe toutes les facultés de l’âme.

\section{Le plaisir et la douleur en sont le seul mobile.}

Enfin, si nous considérons qu'il n'est point de sensations absolument différentes, nous conclurons encore que les différents degrés de 
plaisir et de peine sont la loi, suivant laquelle le germe de tout ce que nous sommes s'est développé, pour produire toutes nos facultés.

Ce principe peut prendre les noms de besoin, d'étonnement et d'autres, que nous lui donnerons encore ; mais il est toujours le même : car nous sommes toujours mus par le plaisir ou par la douleur, dans tout ce que le besoin ou l'étonnement nous fait faire.

En effet, nos premières idées ne sont que peine, ou plaisir. Bientôt d'autres leur succèdent, et donnent lieu à des comparaisons, d'où naissent nos premiers besoins, et nos premiers désirs. Nos recherches, pour les satisfaire, font acquérir d'autres idées, qui produisent encore de nouveaux désirs. L'étonnement, qui contribue à nous faire sentir vivement tout ce qui nous arrive d'extraordinaire, augmente de temps en temps l'activité de nos facultés ; et il se forme une chaîne, dont les anneaux sont tour-à-tour idées et désirs ; et qu'il suffit de suivre, pour découvrir le progrès de toutes les connaissances de l’homme.

\section{On peut appliquer aux autres sens tout ce qui} vient d'être dit sur l'odorat.

Presque tout ce que j'ai dit sur les facultés de l'âme, en traitant de l'odorat, j'aurais pu le dire, en commençant par tout autre sens : il est aisé de leur en faire l'application. Il ne me reste qu'à examiner ce qui est plus particulier à chacun d'eux. 


\section{Chapitre VIII}

\section{D’un homme borné au sens de l'ouïe}

La statue bornée au sens de l'ouïe est tout ce qu'elle entend.

Bornons notre statue au sens de l'ouïe, et raisonnons, comme nous avons fait, quand elle n'avait que celui de l'odorat.

Lorsque son oreille sera frappée, elle deviendra la sensation qu'elle éprouvera. Elle sera comme l'écho dont Ovide dit : sonus est qui vivit in illa ; c'est le son qui vit en elle. Ainsi nous la transformerons, à notre gré, en un bruit, un son, une symphonie : car elle ne soupçonne pas qu'il existe autre chose qu'elle. L'ouïe ne lui donne l'idée d'aucun objet, situé à une certaine distance. La proximité, ou l'éloignement des corps sonores ne produit à son égard qu'un son plus fort ou plus faible : elle en sent seulement plus ou moins son existence.

\section{Deux sortes de sensations de l'ouïe.}

Les corps font sur l'oreille deux sortes de sensations ${ }^{15}$ : l'une est le son proprement dit, l'autre est le bruit.

15 1. On a remarqué que, dans la résonnance des corps sonores, le son dominant est accompagné de deux autres, qui ont avec lui un rapport déterminé, et soumis au calcul. On les appelle les harmoniques du son dominant. Ils se font entendre à la douzième et à la dix-septième, et l'on en fait la tierce et la quinte. Une oreille bien organisée est capable de saisir ces rapports, et c'est pour cela que l'on dit qu'elle apprécie les sons. On peut donc définir le son proprement dit, un son appréciable.

Le bruit au contraire résulte de plusieurs sons qui n’ont point d'harmoniques communes; c'est une multitude de sons dominants et d'harmoniques qui se confondent : on peut donc le définir un son inappréciable.

Imaginons une dizaine de violons à l'unisson. S'ils font tous résonner en 
L'oreille est organisée, pour saisir un rapport déterminé entre un son et un son ; mais elle ne peut saisir entre un bruit et un bruit, qu'un rapport vague. Le bruit est à-peu-près au sens de l'ouïe, ce qu'est une multitude d'odeurs à celui de l'odorat.

La statue ne distingue plusieurs bruits

qu'autant qu'ils se succèdent.

Si au premier instant, plusieurs bruits se font entendre ensemble à notre statue, le plus fort enveloppera le plus faible ; ils se mêleront si bien, qu'il n'en résultera pour elle qu'une simple manière d'être, où ils se confondront.

S’ils se succèdent, elle conserve le souvenir de ce qu'elle a été. Elle distingue ses différentes manières d'être, elle les compare, elle en juge, et elle en forme une suite, que sa mémoire retient dans l'ordre où elles ont été comparées, supposé que cette suite l'ait frappée à plusieurs reprises. Elle reconnaîtra donc ces bruits, lorsqu'ils se succéderont encore ; mais elle ne les reconnaîtra plus, lorsqu'ils se feront entendre en même temps. Il faut raisonner à ce sujet, comme nous avons fait sur les odeurs.

\section{Il en est de même des sons.}

Quant aux sons proprement dits, l'oreille étant organisée pour en sentir exactement les rapports, elle y apporte un discernement plus fin et plus étendu. Ses fibres semblent se partager les vibrations des corps sonores, et elle peut entendre distinctement plusieurs sons à-la-fois. Cependant il suffit de considérer qu'elle n'a pas tout ce discernement dans les hommes qui ne sont point exercés à la musique, pour être au moins convaincu que notre statue ne distinguera pas au premier instant deux sons qu'elle entendra ensemble.

même temps la même corde, ils rendent ensemble un son proprement dit, un son appréciable ; parce qu'on en peut déterminer la tierce et la quinte. Mais si nous les supposons tous discordants, ils ne feront que du bruit, parce que le son total qu'ils font entendre, n'a point d'harmonique. Le même mi et le même sol, qui sont les harmoniques de l'ut de l'un de ces violons, ne seront pas les harmoniques des ut que les autres rendent. C'est donc la confusion de plusieurs sons, qui fait le bruit. 
Mais les démêlera-t-elle, si elle les a étudiés séparément ? C’est ce qui ne me paraît pas vraisemblable : quoique son oreille soit par son mécanisme capable d'en faire la différence, les sons ont tant d'analogie entre eux, qu'il y a lieu de présumer, que n'étant pas aidée par les jugements, qui accoutument à les rapporter à des corps différents, elle continuera encore à les confondre.

Elle acquiert les mêmes facultés qu'avec

l'odorat.

Quoi qu'il en soit, les degrés de plaisir et de peine lui feront acquérir les mêmes facultés qu'elle a acquises avec l'odorat : mais il y a sur ce point quelques remarques particulières à faire.

Les plaisirs de l'oreille consistent principalement dans l'harmonie.

Premièrement, les plaisirs de l'oreille consistent principalement dans la mélodie, c'est-à-dire, dans une succession de sons harmonieux auxquels la mesure donne différents caractères. Les désirs de notre statue ne se borneront donc pas à avoir un son pour objet, et elle souhaitera de redevenir un air entier.

Cette harmonie cause une émotion qui ne suppose point d'idées acquises.

En second lieu, ils ont un caractère bien différent de ceux de l'odorat. Plus propre à émouvoir que les odeurs, les sons donneront, par exemple, à notre statue cette tristesse, ou cette joie, qui ne dépendent point des idées acquises, et qui tiennent uniquement à certains changements qui arrivent au corps ${ }^{16}$.

16 Il y a dans la musique les plaisirs d'imitation, lorsqu'elle imite le chant des oiseaux, le tonnerre, les tempêtes, nos soupirs, nos plaintes, nos cris de joie ; et que, par sa mesure, elle invite notre corps à prendre les attitudes et les mouvements des différentes passions. Notre statue n’est pas faite pour ces sortes de plaisirs ; parce qu'ils supposent des jugements et des habitudes dont elle n'est point capable. Mais indépendamment de cette imitation, la musique transmet au cerveau des impressions qui passent dans tout le corps, et qui y produisent des émotions où notre statue ne peut manquer de trouver du plaisir ou de la peine. 
Ces plaisirs sont, comme ceux de l'odorat, susceptibles de différents degrés.

En troisième lieu, ils commencent, ainsi que ceux de l'odorat, à la plus légère sensation. Le premier bruit, quelque faible qu'il puisse être, est donc un plaisir pour notre statue. Que le bruit augmente, le plaisir augmentera, et ne cessera que quand les vibrations offenseront le tympan.

Les plus vifs supposent une oreille exercée.

Quant à la musique, elle lui plaira davantage, suivant qu'elle sera en proportion avec le peu d'exercice de son oreille. D'abord des chants simples et grossiers seront capables de la ravir. Si nous l'accoutumons ensuite peu à peu à de plus composés, l'oreille se fera une habitude de l'exercice, qu'ils demandent : elle connaîtra de nouveaux plaisirs.

Et tous, une oreille bien organisée.

Au reste, ce progrès n'est que pour les oreilles bien organisées. Si les fibres ne sont point entre elles dans de certains rapports, l'oreille sera fausse, comme un instrument mal monté. Plus ce vice sera considérable, moins elle sera sensible à la musique : elle pourra même ne l'être pas plus qu'au bruit.

La statue peut parvenir à distinguer un bruit et un chant qui se font entendre ensemble.

En quatrième lieu, le plaisir d'une succession de sons étant si supérieur à celui d'un bruit continu, il y a lieu de conjecturer, que si la statue entend en même temps un bruit et un air, dont l'un ne domine point sur l'autre, et qu'elle a appris à connaître séparément, elle ne les confondra pas.

$\mathrm{Si}$, au premier moment de son existence, elle les avait entendus ensemble, elle n'en eût pas fait la différence. Car nous savons par nousmêmes, que nous ne démêlons dans les impressions des sens que ce que nous y avons pu remarquer; et que nous n'y remarquons que les idées auxquelles nous avons successivement donné notre attention. Mais si notre statue, ayant été tour-à-tour un chant et le bruit d'un 
ruisseau, s'est fait une habitude de distinguer ces deux manières d'être, et de partager entre elles son attention ; elles sont, ce me semble, trop différentes pour se confondre encore, toutes les fois qu'elle les éprouve ensemble ; surtout si, comme je le suppose, aucune ne domine. Elle ne peut donc s'empêcher de remarquer qu'elle est tout-àla-fois ce bruit et ce chant, dont elle se souvient, comme de deux modifications, qui se sont auparavant succédées.

Le principe sur lequel je fonde ce que je présume ici, recevra un nouveau jour dans la suite de cet ouvrage ; parce que j'aurai occasion de l'appliquer à des exemples encore plus sensibles. Nous verrons comment par la manière, dont nous jugeons de nos sensations, nous n'y savons distinguer que ce que les circonstances nous ont appris à y remarquer ; que tout le reste est confus à notre égard, et que nous n'en conservons non plus d'idées, que si nous n'en avions eu aucun sentiment. C'est une des causes, qui fait qu'avec les mêmes sensations, les hommes ont des connaissances si différentes. Ce germe est partout le même : mais il reste informe chez les uns ; il se développe, se nourrit, et s’accroît chez les autres.

Une suite de sons se lient mieux dans la mémoire, qu'une suite de bruits.

Enfin, puisque les bruits sont à l'oreille, ce que les odeurs sont au nez, la liaison en sera dans la mémoire la même que celle des odeurs. Mais les sons ayant, par leur nature, et par celle de l'organe, un lien beaucoup plus fort, la mémoire en conservera plus facilement la succession. 


\section{Chapitre IX}

\section{De l'odorat et de l'ouïe réunis}

Ces deux sens réunis ne donnent l'idée d'aucune chose extérieure.

Dès que ses sens pris séparément, ne donnent pas à notre statue l'idée de quelque chose d'extérieur, ils ne la lui donneront pas davantage après leur réunion. Elle ne soupçonnera pas qu'elle ait deux organes différents.

D'abord la statue ne distingue pas les sons des odeurs qui viennent à elle en même temps.

Si même, au premier moment de son existence, elle entend des sons, et sent des odeurs, elle ne saura pas encore distinguer en elle deux manières d'être. Les sons et les odeurs se confondront, comme s'ils n'étaient qu'une modification simple. Car nous venons d'observer qu'elle ne distingue dans ses sensations que les idées qu'elle a eu occasion de remarquer chacune en particulier.

Elle apprend ensuite à les distinguer.

Mais si elle a considéré les sensations de l'ouïe séparément de celles de l'odorat, elle sera capable de les distinguer, lorsqu'elle les éprouvera ensemble : car pourvu que le plaisir de jouir de l'une, ne la détourne pas entièrement du plaisir de jouir de l'autre, elle reconnaîtra qu'elle est tout-à-la-fois ce qu'elle a été tour-à-tour. La nature de ces sensations ne les porte pas à se confondre comme deux odeurs : elles différent trop, pour n'être pas distinguées, au souvenir qui reste de chacune. C'est donc à la mémoire que la statue doit l'avantage de distinguer les impressions qui lui sont transmises à-la-fois par des organes différents. 
Son être lui paraît acquérir une double existence.

Alors il lui semble que son être augmente, et qu'il acquiert une double existence. Voilà donc bien du changement dans ses jugements d'habitude ; car avant la réunion de l'ouïe à l'odorat, elle n'avait point imaginé qu'elle pût être à-la-fois de deux manières si différentes.

Sa mémoire est plus étendue qu'avec un seul sens.

Il est évident qu'elle s'acquerra les mêmes facultés, que lorsqu'elle a eu séparément ces deux sens. Sa mémoire y gagnera, en ce que la chaîne des idées en sera plus variée et plus étendue. Tantôt un son lui rappellera une suite d'odeurs ; tantôt une odeur lui rappellera une suite de sons. Mais il faut remarquer que ces deux espèces de sensations étant réunies, sont sujettes à la même loi qu'avant leur réunion ; c'està-dire, que les plus vives peuvent quelquefois faire oublier les autres, et empêcher qu'elles soient remarquées, au moment même qu'elles ont lieu.

Elle forme plus d'idées abstraites.

Il me semble encore que la statue peut avoir plus d'idées abstraites qu'avec un seul sens. Elle ne connaissait en général que deux manières d'être, l'une agréable, l'autre désagréable: mais actuellement qu'elle distingue les sons des odeurs, elle ne peut s'empêcher de les considérer, comme deux espèces de modification. Peut-être encore le bruit lui paraît-il si différent des sons harmonieux, que si on pouvait lui faire comprendre que ses sensations lui sont transmises par des organes; elle pourrait bien imaginer avoir trois sens; un pour les odeurs, un autre pour le bruit, et un troisième pour les sons harmonieux. 


\section{Chapitre X}

\section{Du goût seul, et du goût joint à l'odorat et à l'ouïe}

La statue acquiert les mêmes facultés qu’avec

l'odorat.

Ne donnant de sensibilité qu’à l'intérieur de la bouche de notre statue, je ne saurais lui faire prendre aucune nourriture : mais je suppose que l'air lui apporte à mon gré toutes sortes de saveurs, et soit propre à la nourrir toutes les fois que je le jugerai nécessaire.

Elle acquerra les mêmes facultés qu'avec l'ouïe ou l'odorat; et parce que sa bouche est aux saveurs, ce que le nez est aux odeurs, et l'oreille au bruit ; plusieurs saveurs réunies lui paraîtront comme une seule, et elle ne les distinguera, qu'autant qu'elles se succéderont.

Le goût contribue plus que l'odorat et que l'ouîe à son bonheur et à son malheur.

Le goût peut ordinairement contribuer plus que l'odorat, à son bonheur et à son malheur : car les saveurs affectent communément avec plus de force que les odeurs.

Il y contribue même encore plus que les sons harmonieux; parce que le besoin de nourriture lui rend les saveurs plus nécessaires, et par conséquent les lui fait goûter avec plus de vivacité. La fin pourra la rendre malheureuse : mais dès qu'elle aura remarqué les sensations propres à l'apaiser, elle y déterminera davantage son attention, les désirera avec plus de violence, et en jouira avec plus de délice.

Discernement qu'elle fait des sensations qu'ils lui transmettent.

Si nous réunissons le goût à l'ouïe et à l'odorat, la statue parviendra à démêler les sensations qu'ils lui transmettent à-la-fois, lors- 
qu'elle aura appris à les connaître séparément ; pourvu néanmoins que son attention se partage à-peu-près également entre elles : ainsi voilà son existence en quelque sorte triplée.

Il est vrai qu'il ne lui sera pas toujours aussi aisé de faire la différence d'une saveur à une odeur, que d'une saveur à un son. L'odorat et le goût ont une si grande analogie, que leurs sensations doivent quelquefois se confondre ${ }^{17}$.

\section{Le goût peut nuire aux autres sens.}

Comme nous venons de voir que les saveurs doivent l'intéresser plus que toute autre sensation ; elle s'en occupera d'autant plus, que sa faim sera plus grande. Le goût pourra donc nuire aux autres sens, jusqu'à la rendre insensible aux odeurs et à l'harmonie.

\section{Avantages résultants de la réunion de ces}

sens.

La réunion de ces sens étendra, et variera davantage la chaîne de ses idées, augmentera le nombre de ses désirs, et lui fera contracter de nouvelles habitudes.

\section{Doute sur leurs effets.}

Cependant il est très difficile de déterminer jusqu'à quel point la statue pourra distinguer les manières d'être qu'elle leur doit. Peut-être son discernement est-il moins étendu que je ne l'imagine ${ }^{18}$, peut-être l'est-il davantage. Pour en juger, il faudrait se mettre tout-à-fait à sa place, et se dépouiller entièrement de toutes ses habitudes : mais je ne me flatte pas d'y avoir toujours réussi.

L'habitude de rapporter chaque espèce de sensation à un organe particulier, doit beaucoup contribuer à nous en faire faire la différence : sans elle, peut-être que nos sensations seraient une espèce de

17 Il n’y a personne qui n'ait pu remarquer qu'il est quelquefois porté à attribuer à un mets, dont il mange, les odeurs qui frappent son odorat. Mais ce qui prouve encore cette analogie, c'est qu'on a plus de goût à proportion qu'on a l'odorat plus fin.

18 C’était le sentiment de Mademoiselle Ferrand. 
chaos pour nous. En ce cas, le discernement de la statue serait fort borné.

Mais il faut remarquer que l'incertitude, ou la fausseté même de quelques conjectures, ne saurait nuire au fond de cet ouvrage. Quand j'observe cette statue, c'est moins pour m'assurer de ce qui se passe en elle, que pour découvrir ce qui se passe en nous. Je puis me tromper, en lui attribuant des opérations, dont elle n'est pas encore capable ; mais de pareilles erreurs ne tirent pas à conséquence, si elles mettent le lecteur en état d'observer comment ces opérations s'exécutent en lui-même. 


\section{Chapitre XI}

\section{D’un homme borné au sens de la vue}

Préjugés et considérations qui le combattent.

Il paraîtra sans doute extraordinaire à bien des lecteurs, de dire que l'œil est par lui-même incapable de voir un espace hors de lui. Nous nous sommes fait une si grande habitude de juger, à la vue des objets qui nous environnent, que nous n'imaginons pas comment nous n'en aurions pas jugé, au premier moment que nos yeux se sont ouverts à la lumière.

La raison a bien peu de force, et ses progrès sont bien lents, lorsqu'elle a à détruire des erreurs, dont personne n'a pu s'exempter, et qui, ayant commencé avec le premier développement des sens, cachent leur origine dans des temps dont nous ne conservons aucun souvenir. D'abord on pense que nous avons toujours vu comme nous voyons ; que toutes nos idées sont nées avec nous; et nos premières années sont comme cet âge fabuleux des poètes, où l'on suppose que les dieux ont donné à l'homme toutes les connaissances, qu'il ne se souvient pas d'avoir acquises par lui-même.

Si un philosophe soupçonne que toutes nos connaissances pourraient bien tirer leur origine des sens, aussitôt les esprits se révoltent contre une opinion qui leur paraît si étrange. Quelle est la couleur de la pensée, lui demande-t-on, pour venir à l'âme par la vue ? Qu'elle en est la saveur, qu'elle en est l'odeur, etc. Pour être due au goût, à l'odorat, etc. ? Enfin, on l'accable de mille difficultés de cette sorte, avec toute la confiance que donne un préjugé généralement reçu. Le philosophe, qui s'est hâté de prononcer, avant d'avoir démêlé la génération de toutes nos idées, est embarrassé ; on ne doute pas que ce ne soit une preuve de la fausseté de son sentiment. 
La philosophie fait un nouveau pas : elle découvre que nos sensations ne sont pas les qualités mêmes des objets, et qu'au contraire elles ne sont que des modifications de notre âme. Elle examine chaque sensation en particulier ; et comme elle trouve peu de difficultés dans cette recherche, elle paraît à peine faire une découverte.

De là il était aisé de conclure que nous n’apercevons rien qu'en nous-mêmes ; et que par conséquent un homme borné à l'odorat, n'eût été qu’odeur ; borné au goût, saveur ; à l'ouïe, bruit ou son ; à la vue, lumière et couleur. Alors le plus difficile eût été d'imaginer comment nous contractons l'habitude de rapporter au-dehors des sensations qui sont en nous. En effet, il paraît bien étonnant qu'avec des sens, qui n'éprouvent rien qu'en eux-mêmes, et qui n'ont aucun moyen pour soupçonner un espace au-dehors, on pût rapporter ses sensations aux objets qui les occasionnent. Comment le sentiment peut-il s'étendre au-delà de l'organe qui l'éprouve, et qui le limite ?

Mais en considérant les propriétés du toucher, on eût reconnu qu'il est capable de découvrir cet espace, et d'apprendre aux autres sens à rapporter leurs sensations aux corps qui y sont répandus. Dès lors les personnes mêmes, que le préjugé éloignait davantage de cette vérité, eussent commencé à former au moins quelque doute. On serait tombé d'accord qu'avec l'odorat, ou le goût, on ne se serait cru qu'odeur, ou saveur. L'ouïe eût souffert un peu plus de difficulté, par l'habitude où nous sommes d'entendre le bruit, comme s'il était hors de nous. Mais ce sens a tant de peine à juger des distances et des situations, et il s'y trompe si souvent, qu'on fut enfin convenu qu'il n'en juge point par lui-même. On l'eût regardé comme un élève qui a mal retenu les leçons du toucher.

Mais la vue, comment aura-t-elle pu être instruite par le tact, elle qui juge des distances auxquelles il ne peut atteindre ; elle qui embrasse en un instant des objets, qu'il ne parcourt que lentement, ou dont même il ne peut jamais saisir l'ensemble?

L'analogie eût pu faire présumer qu'il doit en être d'elle comme des autres sens : l'impression de la lumière, la sensation étant toute dans les yeux, l'on pouvait conjecturer qu'ils doivent ne voir qu'en eux-mêmes, lorsqu'ils n'ont point encore appris à rapporter leurs sensations sur les objets. En effet, s'ils ne voyaient que comme ils sen- 
tent, pourraient-ils soupçonner qu'il y a un espace au-dehors, et dans cet espace des objets qui agissent sur eux ?

On eût donc supposé qu'ils n’ont, par eux-mêmes, connaissance que de la lumière et des couleurs ; et après avoir dans cette hypothèse rendu raison de tous les phénomènes ; après avoir expliqué comment avec le secours du tact, ils parviennent à juger des objets qui sont dans l'espace ; il n'eût manqué que des expériences, pour achever de détruire tous nos préjugés.

On doit rendre à M. Molyneux la justice d'avoir le premier formé des conjectures sur la question que nous traitons. Il communiqua sa pensée à un philosophe ; c'était le seul moyen de se faire un partisan. Locke convint avec lui qu'un aveugle-né, dont les yeux s'ouvriraient à la lumière, ne distinguerait pas à la vue un globe d'un cube. Cette conjecture a été depuis confirmée par les expériences de M. Cheselden, auxquelles elle a donné occasion; et il me semble qu'on peut aujourd'hui démêler à-peu-près ce qui appartient aux yeux, et ce qu'ils doivent au tact.

La statue n'aperçoit les couleurs que comme des manières d'être d'elle-même.

Je crois donc être autorisé à dire que notre statue ne voit que de la lumière et des couleurs, et qu'elle ne peut pas juger qu'il y a quelque chose hors d'elle.

Cela étant, elle n'aperçoit dans l'action des rayons, que des manières d'être d'elle-même. Elle est avec ce sens, comme elle a été avec ceux dont nous avons déjà examiné les effets; et elle acquiert les mêmes facultés.

\section{Au premier instant, elle les voit confusément.}

Si dès le premier instant elle aperçoit également plusieurs couleurs, il me semble qu'elle n'en peut encore remarquer aucune en particulier: son attention trop partagée les embrasse confusément. Voyons comment elle peut apprendre à les démêler. 
Comment elle les discerne ensuite les unes après les autres.

L'œil est de tous les sens celui dont nous connaissons le mieux le mécanisme. Plusieurs expériences nous ont appris à suivre les rayons de la lumière jusque sur la rétine ; et nous savons qu'ils y font des impressions distinctes. A la vérité, nous ignorons comment ces impressions se transmettent par le nerf optique jusqu'à l'âme. Mais il paraît hors de doute qu'elles y arrivent sans confusion : car l'auteur de la nature aurait-il pris la précaution de les démêler avec tant de soin sur la rétine, pour permettre qu'elles se confondissent à quelques lignes au-delà ? Et si d'ailleurs cela arrivait, comment l'âme apprendrait-elle jamais à en faire la différence ?

Les couleurs sont donc par leur nature des sensations, qui tendent à se démêler ; et voici comment j'imagine que notre statue parviendra à en remarquer un certain nombre.

Parmi les couleurs, qui se répandent au premier instant dans son œil, et qui en occupent le fond, il peut y en avoir une qu'elle distingue d'une manière particulière, qu'elle voit comme à part : ce sera celle à laquelle le plaisir déterminera son attention avec un certain degré de vivacité. Si elle ne la remarquait pas plus que les autres, elle ne la démêlerait point encore. C'est ainsi que nous ne discernerions rien dans une campagne, où nous voudrions tout voir à-la-fois et également.

Si elle en pouvait considérer avec la même vivacité deux ensemble, elle les remarquerait avec la même facilité qu'une seule ; si elle en pouvait considérer trois de la sorte, elle les remarquerait également. Mais c'est de quoi elle ne me paraît pas encore capable : il faut que le plaisir de les considérer l'une après l'autre, la prépare au plaisir d'en considérer plusieurs à-la-fois.

Il est vraisemblable qu'elle est par rapport à deux ou trois couleurs, qui s'offrent à elle avec quantité d'autres, comme nous sommes nousmêmes par rapport à un tableau un peu composé, et dont le sujet ne nous est pas familier. D'abord nous en apercevons les détails confusément. Ensuite nos yeux se fixent sur une figure, puis sur une autre ; et ce n'est qu'après les avoir remarquées successivement, que nous parvenons à juger de toutes ensemble. 
La vue confuse du premier coup-d'œil n'est pas l'effet d'un nombre d'objets absolu et déterminé ; en sorte que ce qui est confus pour moi, doive l'être pour tout autre. Elle est l'effet d'une multitude trop grande par rapport au peu d'exercice de mes yeux. Un peintre et moi nous voyons également toutes les parties d'un tableau: mais tandis qu'il les démêle rapidement, je les découvre avec tant de peine, qu'il me semble que je voie à chaque instant ce que je n'avais point encore vu.

Ainsi donc qu'il y a dans ce tableau plus de choses distinctes pour ses yeux, et moins pour les miens ; notre statue, parmi toutes les couleurs, qu'elle voit au premier instant, n'en peut vraisemblablement remarquer qu'une seule, puisque ses yeux n'ont point encore été exercés.

Alors, quoique d'autres couleurs se répandent distinctement sur sa rétine, et que par conséquent elle les voie ; elles sont aussi confuses à son égard, que si elles se confondaient réellement.

Tant qu'elle est toute entière à la couleur qu'elle remarque, elle n'a donc proprement aucune connaissance des autres.

Cependant ses yeux se fatiguent, soit parce que cette couleur agit avec vivacité, soit parce qu'ils ne sauraient demeurer sans quelque effort dans la situation qui les fixe sur elle. Ils en changent donc par un mouvement machinal : ils en changent encore, s'ils sont par hasard frappés d'une couleur trop vive pour leur plaire ; et ils ne s'arrêtent que lorsqu'ils en rencontrent une qui leur est plus agréable, parce qu'elle est un repos pour eux.

Après quelque temps, ils se fatiguent encore, et ils passent à une couleur moins vive. Ainsi ils arriveront par degrés à mettre leur plus grand plaisir à ne remarquer que du noir. Enfin, la lassitude peut être portée à un tel point, qu'ils se fermeront tout-à-fait à la lumière.

Si notre statue, ayant démêlé les couleurs dans cet ordre successif, n'en pouvait jamais remarquer plusieurs en même temps, elle serait précisément avec la vue, comme elle a été avec l'odorat. Car quoique jusqu'ici elle en ait toujours vu plusieurs ensemble, toutes celles qu'elle n’a pas remarquées, sont, à son égard, comme si elle ne les 
avait point vues : elle n'en peut tenir aucun compte. Mais il me paraît qu'elle doit apprendre à en démêler plusieurs à-la-fois.

\section{Comment elle en discerne plusieurs à-la-fois.}

Le rouge, je le suppose, est la première couleur, qui l'a frappée davantage, et qu'elle a remarquée. Son œil étant fatigué, il change de situation, et il rencontre une autre couleur, du jaune, par exemple : elle se plaît à cette nouvelle manière d'être ; mais elle n'oublie pas le rouge, ni le plaisir qu'il lui a fait. Son attention se partage donc entre ces deux couleurs : si elle remarque le jaune, comme une manière d'être qu'elle éprouve actuellement; elle remarque le rouge, comme une manière d'être qu'elle a éprouvée.

Mais le rouge ne peut pas attirer son attention, et continuer de ne lui paraître que comme une manière d'être, qui n'est plus ; si la sensation, comme je le suppose, lui en est aussi présente que celle du jaune. Après s'être rappelé qu'elle a été rouge et jaune successivement ; elle remarque donc qu'elle est rouge et jaune tout-à-la-fois.

Qu'ensuite son œil fatigué se porte sur une troisième couleur, sur du vert, par exemple, son attention déterminée à cette manière d'être, se détourne des deux premières. Cependant elle n'y est pas déterminée, au point de lui faire tout-à-fait oublier ce qu'elle a été. Elle remarque donc encore le rouge et le jaune, comme deux manières d'être, qui ont précédé.

Ce souvenir prend sur l'attention, à proportion que l'organe, fixé sur le vert, se fatigue. Insensiblement il y a à-peu-près autant de part que la couleur actuellement remarquée : ainsi la statue démêle qu'elle a été du rouge et du jaune avec la même vivacité qu'elle démêle qu'elle est du vert. Dès lors elle remarque qu'elle est tout-à-la-fois ces trois couleurs. Et comment se bornerait-elle à en considérer deux comme passées; lorsque ces sensations sont toutes trois en même temps dans ses yeux, et qu'elles y sont d'une manière distincte ?

C'est donc par le secours de la mémoire que l'œil parvient à remarquer jusqu'à deux ou trois couleurs, qui se présentent ensemble. Si lorsqu'il remarque la seconde, la première s'oubliait totalement, jamais il ne parviendrait à juger qu'il est tout-à-la-fois de deux manières. Mais dès que le souvenir en reste, l'attention se partage entre 
l'une et l'autre ; et aussitôt qu'il a remarqué qu'il a été successivement de deux manières, il juge qu'il est de deux tout-à-la-fois.

Bornes de son discernement à ce sujet.

Comme nous lui avons appris à connaître successivement trois couleurs, nous lui apprendrons à en connaître un plus grand nombre. Mais dans toute cette succession il ne s'en représentera jamais que trois distinctement : car les idées de notre statue sur les nombres ne sont pas plus étendues, qu'elles l'étaient avec l'odorat.

Si nous lui offrons ensuite toutes ces couleurs ensemble, elle n'en démêlera également que trois à-la-fois, et elle ne pourra déterminer le nombre des autres. Ayant démontré que l'œil a besoin de la mémoire pour les distinguer, il est hors de doute qu'il n'en distinguera pas plus que la mémoire même.

Elle a avec ce sens un moyen de plus pour se procurer ce qu'elle désire.

Notre statue portant la vue d'une couleur à une autre, ne jouit pas toujours de la manière d'être, qu'elle se souvient lui avoir été plus agréable. Son imagination faisant effort, pour lui représenter vivement l'objet de son désir, ne peut manquer d'agir sur les yeux. Elle y produit donc à leur insu un mouvement, qui leur fait parcourir plusieurs couleurs, jusqu'à ce qu'ils aient rencontré celle qu'ils cherchent. La statue a par conséquent avec ce sens un moyen de plus qu'avec les précédents, pour obtenir la jouissance de ce qu'elle désire. Il se pourra même qu'ayant d'abord retrouvé, comme par hasard, une couleur, ses yeux prennent l'habitude du mouvement propre à la leur faire retrouver encore ; et cela arrivera, pourvu que les objets qui leur sont présents, ne changent pas de situation.

\section{Comment elle se sent étendue.}

Une sensation de son ne saurait offrir de l'étendue à l'âme qui en est modifiée, parce qu'un son n'est pas étendu. Il n'en est pas de même d'une sensation de couleur : elle offre de l'étendue à l'âme qu'elle modifie, parce qu'elle est étendue elle-même. C'est un fait qu'on ne peut révoquer en doute : l'observation le démontre. Aussi est-il im- 
possible de concevoir une couleur sans étendue, comme il est impossible de concevoir un son étendu.

Dès que chaque couleur est étendue, plusieurs couleurs contiguës forment nécessairement un continu de plusieurs parties étendues et distinctes les unes des autres.

Ce phénomène est une surface colorée. C’est ainsi du moins que nous l'apercevons nous-mêmes. Notre statue, lorsqu'elle juge qu'elle est à-la-fois plusieurs couleurs, se sentirait donc comme une surface colorée.

L'idée de l'étendue suppose la perception de plusieurs choses, qui, étant les unes hors des autres, sont contiguës, et par conséquent chacune étendues : car des choses inétendues ne sauraient être contiguës. On ne peut pas refuser cette perception à la statue : car elle sent qu'elle se répète hors d'elle-même, autant de fois qu'il y a de couleurs qui la modifient. En tant qu'elle est le rouge, elle se sent hors du vert ; en tant qu'elle est le vert, elle se sent hors du rouge, et ainsi du reste.

Elle se sent donc comme une étendue colorée : mais cette étendue n’est pour elle ni une surface, ni aucune grandeur déterminée.

Elle n'est pas une surface, parce que l'idée de surface suppose l’idée de solide, idée qu'elle n’a pas et qu'elle ne peut avoir.

Elle n’est pas non plus une grandeur déterminée : car une pareille grandeur est une étendue renfermée dans des limites qui la circonscrivent. Or, le moi de la statue ne saurait se sentir circonscrit dans des limites. Il est à-la-fois toutes les couleurs qui le modifient en même temps ; et puisqu'il ne voit rien au-delà, il ne saurait s'apercevoir comme circonscrit. Parce qu'il est modifié à-la-fois par plusieurs couleurs, et qu'il se retrouve également dans chacune, il se sent comme étendu, et parce qu'il n'aperçoit rien qui le circonscrive, il n'a de son étendue qu'un sentiment vague : c'est pour lui une étendue sans bornes. Il lui semble qu'il se répète sans fin, et ne connaissant rien audelà des couleurs qu'il croit être, il est par rapport à lui, comme s'il était immense : il est partout, il est tout.

Mais dans son étendue qui lui paraît immense, les différentes couleurs se terminent mutuellement, elles dessinent donc des figures. Or 
la statue croira-t-elle encore être ces figures ? A-t-elle des idées de figures, aussitôt qu'elle a des sensations de couleur?

Une sensation renferme telle et telle idée : donc nous avons ces idées aussitôt que nous avons cette sensation. Voilà une conclusion que les mauvais métaphysiciens ne manquent jamais de tirer. Cependant nous n'avons pas toutes les idées que nos sensations renferment ; nous n'avons que celles que nous y savons remarquer. Ainsi nous voyons tous les mêmes objets; mais parce que nous n'avons pas le même plaisir, le même intérêt à les observer, nous en avons chacun des idées bien différentes. Vous remarquez ce qui m'échappe, et souvent lorsque vous en pouvez rendre un compte exact, je suis moimême comme si je n’avais rien vu.

Or, la lumière et les couleurs étant le côté le plus sensible, par où la statue se connaît, par où elle jouit d'elle-même ; elle sera plus portée à considérer ses modifications, comme éclairées et colorées, que comme figurées. Toute occupée à juger des couleurs, par les nuances, qui les distinguent, elle ne pensera donc pas aux différentes manières, dont nous les supposons terminées.

D'ailleurs il ne suffit pas à l'œil de voir toute une figure, pour s'en former une idée; comme il lui suffit de voir une couleur, pour la connaître. Il ne saisit l'ensemble de la plus simple, qu'après en avoir parcouru toutes les parties. Il lui faut un jugement pour chacune en particulier, et un autre jugement pour les réunir : il faut se dire, voilà un côté, en voilà un second, en voilà un troisième ; voilà l'intervalle qui les sépare, et de tout cela résulte ce triangle.

Ainsi donc que les yeux n’ont appris à démêler trois couleurs à-lafois, que parce que les ayant considérées successivement, ils les remarquent dans l'impression qu'elles font ensemble: de même, ils n’apprendront à démêler les trois côtés d'un triangle, qu'autant que les ayant remarqués l'un après l'autre, ils les remarqueront tous ensemble, et jugeront de la manière dont ils se réunissent. Mais c'est là un jugement que la statue n'aura point occasion de former.

Les figures, nous le supposons, sont renfermées dans les sensations qu'elle éprouve. Mais notre expérience nous démontre assez que nous n'avons pas toutes les idées que nos sensations portent avec elles. Nos connaissances se bornent uniquement aux idées que nous avons appris 
à remarquer : nos besoins sont la seule cause qui détermine notre attention aux unes plutôt qu'aux autres ; et celles qui demandent un plus grand nombre de jugements, sont aussi celles que nous acquérons les dernières. Or, je n'imagine pas quelle sorte de besoin pourrait engager notre statue à former tous les jugements nécessaires, pour avoir l'idée de la figure la plus simple.

D’ailleurs quel heureux hasard réglerait le mouvement de ses yeux, pour leur en faire suivre le contour ? Et lors même qu'ils le suivraient, comment pourrait-elle s'assurer de ne pas passer continuellement d'une figure à une autre ? à quoi pourra-t-elle juger que trois côtés, qu'elle a vus l'un après l'autre, forment un triangle ? Il est bien plus vraisemblable que sa vue obéissant uniquement à l'action de la lumière, errera dans un chaos de figures : tableau mouvant, dont les parties lui échappent tour-à-tour.

Il est vrai que nous ne remarquons pas les jugements que nous portons, pour saisir l'ensemble d'un cercle, ou d'un carré. Mais nous ne remarquons pas davantage ceux qui nous font voir les couleurs hors de nous. Cependant il sera démontré que cette apparence est l'effet de certains jugements que l'habitude nous a rendu familiers. Qu'on nous offre un tableau fort composé, l'étude que nous en faisons, ne nous échappe pas : nous nous apercevons que nous comptons les personnages, que nous en parcourons les attitudes, les traits, que nous portons sur toutes ces choses une suite de jugements, et que ce n'est qu'après toutes ces opérations, que nous les embrassons d'un même coupd'œil. Or, les yeux de notre statue seraient obligés de faire, pour voir une figure entière, ce que les nôtres font, pour voir un tableau entier. Nous l'avons fait sans doute nous-mêmes la première fois que nous avons appris à voir un carré. Mais aujourd'hui la rapidité avec laquelle nous en parcourons par habitude les côtés, ne nous permet plus de nous apercevoir de la suite de nos jugements. Il est raisonnable de penser, que lorsque nos yeux n'étaient point exercés, ils ont été dans la nécessité de se conduire, pour voir les objets les plus simples, comme ils se conduisent actuellement, pour en voir de plus composés. 
Elle n'a point d'idée de situation ni de mouvement.

Nous ne jugeons des situations, que parce que nous voyons les objets dans un lieu, où ils occupent chacun un espace déterminé ; et nous ne jugeons du mouvement, que parce que nous les voyons changer de situation. Or, la statue ne saurait rien observer de semblable dans les sensations qui la modifient. Si c'est au tact, comme nous le prouverons, à nous faire remarquer dans les couleurs des grandeurs circonscrites, ou des figures, c'est encore à lui à nous faire remarquer dans les couleurs des situations et des mouvements. N'ayant qu'une idée confuse et indéterminée d'étendue, privée de toute idée de figure, de lieu, de situation et de mouvement, la statue sent seulement qu'elle existe de bien des manières. Si plusieurs objets changent de place, sans disparaître à ses yeux, elle continue d'être les mêmes couleurs qu'elle était auparavant. Le seul changement qu'elle peut éprouver, c'est d'être plus sensiblement tantôt l'une tantôt l'autre, suivant les différentes situations, par où le mouvement fait passer les objets : étant tout-à-la-fois par exemple, le jaune, le pourpre et le blanc ; elle sera dans un moment plus le jaune ; dans un autre, plus le pourpre ; et dans un troisième plus le blanc. Elle est toutes les couleurs qu'elle voit : mais elle est plus particulièrement la couleur qu'elle regarde. 


\section{Chapitre XII}

De la vue avec l'odorat, l'ouïe et le goût

Effets produits par la réunion de ces sens.

La réunion de la vue, de l'odorat, de l'ouïe et du goût, augmente le nombre des manières d'être de notre statue : la chaîne de ses idées en est plus étendue et plus variée : les objets de son attention, de ses désirs et de sa jouissance se multiplient ; elle remarque une nouvelle classe de ses modifications, et il lui semble qu'elle aperçoit en elle une multitude d'êtres tout différents. Mais elle continue à ne voir qu'elle, et rien ne la peut encore arracher à elle-même, pour la porter au-dehors.

Ignorance d'où la statue ne peut sortir.

Elle ne soupçonne donc pas qu'elle doive ses manières d'être à des causes étrangères ; elle ignore qu'elles lui viennent par quatre sens. Elle voit, elle sent, elle goûte, elle entend, sans savoir qu'elle a des yeux, un nez, une bouche, des oreilles : elle ne sait pas qu'elle a un corps. Enfin, elle ne remarque qu'elle éprouve ensemble ces différentes espèces de sensations, qu’après les avoir étudiées séparément.

Jugements qu'elle pourrait porter.

Si, supposant qu'elle est continûment la même couleur, nous faisions succéder en elle les odeurs, les saveurs et les sons, elle se regarderait comme une couleur, qui est successivement odoriférante, savoureuse et sonore. Elle se regarderait comme une odeur savoureuse, sonore et colorée, si elle était constamment la même odeur ; il faut faire la même observation sur toutes les suppositions de cette espèce. Car c'est dans la manière d'être, où elle se retrouve toujours, qu'elle 
doit sentir ce moi, qui lui paraît le sujet de toutes les modifications dont elle est susceptible.

Or, quand nous sommes portés à regarder l'étendue, comme le sujet de toutes les qualités sensibles, est-ce parce qu'en effet elle en est le sujet ; ou seulement parce que cette idée étant toujours, par une habitude que nous avons contractée, partout où les autres sont ; et étant la même, quoique les autres varient, elle paraît en être modifiée sans l'être?

De même, quand des philosophes assurent qu'il n'y a que de l'étendue, est-ce qu'il n'existe point d'autre substance ? Est-ce même que l'étendue en est une ? Ou n'en jugent-ils ainsi que parce que cette idée leur est familière, et qu'ils la retrouvent partout ? La statue aurait autant de raison de croire qu'elle n'est qu'une couleur, ou qu'une odeur ; et que cette couleur, ou cette odeur est son être, sa substance. Mais ce n'est pas le lieu de m'arrêter sur de pareils systèmes; et c'est assez les réfuter, que de faire voir qu'ils ne sont pas mieux fondés que les jugements que nous venons de faire porter à notre statue. 


\title{
SECONDE PARTIE
}

\section{Du toucher, ou du seul sens qui juge par lui-même des objets extérieurs}

Table des matières

\author{
Chapitre premier \\ Du moindre degré de sentiment, où l'on peut réduire \\ un homme borné au sens du toucher
}

Sentiment fondamental de la statue.

Notre statue privée de l'odorat, de l'ouïe, du goût, de la vue, et bornée au sens du toucher, existe d'abord par le sentiment qu'elle a de l'action des parties de son corps les unes sur les autres, et surtout des mouvements de la respiration : voilà le moindre degré de sentiment, où l'on puisse la réduire. Je l'appellerai sentiment fondamental ; parce que c'est à ce jeu de la machine que commence la vie de l'animal : elle en dépend uniquement.

Il est susceptible de modification.

Étant exposée ensuite aux impressions de l'air environnant, et de tout ce qui peut la heurter, son sentiment fondamental est susceptible de bien des modifications dans toutes les parties du corps.

Il est la même chose que le moi.

Enfin, nous remarquerons qu'elle pourrait dire moi, aussitôt qu'il est arrivé quelque changement à son sentiment fondamental. Ce sen- 
timent et son moi ne sont, par conséquent, dans l'origine, qu'une même chose ; et pour découvrir ce dont elle peut être capable avec le seul secours du tact, il suffit d'observer les différentes manières, dont le sentiment fondamental, ou le moi, peut être modifié. 
Table des matières

\section{Chapitre II}

\section{Cet homme, borné au moindre degré de sentiment, n'a aucune idée d'étendue, ni de mouvement}

\section{Existence bornée au sentiment fondamental.}

Si notre statue n’est frappée par aucun corps, et si nous la plaçons dans un air tranquille, tempéré, et où elle ne sente ni augmenter, ni diminuer sa chaleur naturelle ; elle sera bornée au sentiment fondamental, et elle ne connaîtra son existence que par l'impression confuse, qui résulte du mouvement auquel elle doit la vie.

\section{Ce sentiment ne donne aucune idée d'étendue.}

Ce sentiment est uniforme et par conséquent simple à son égard ; Elle n’y saurait remarquer les différentes parties de son corps. Elle ne les sent donc point les unes hors des autres et contiguës. Elle est comme si elle n'existait que dans un point, et il ne lui est pas encore possible de découvrir qu'elle est étendue ${ }^{19}$.

19 Nous pouvons nous en convaincre en observant ce qui se passe en nousmêmes.

Une douleur uniforme, qui m’affecte tout le bras, je ne la juge étendue, que parce que je la rapporte à une chose que je sens être étendue.

L'usage que je fais de mon bras, m’apprend à remarquer différentes parties dans sa longueur ; mais il ne m'apprend pas également à remarquer les différentes parties de son diamètre. Aussi je juge bien mieux de la longueur que du volume qu'occupe un sentiment douloureux. Je sais s'il s'étend jusqu'au coude, ou jusqu'au poignet ; et j'ignore s'il affecte le quart, le tiers, la moitié de la grosseur du bras, ou davantage.

Une infinité d'expériences peuvent confirmer qu'on sent la douleur, comme dans un point, toutes les fois qu'on la rapporte à une partie qu'on ne s'est pas fait une habitude de mesurer. Pour découvrir, par exemple, l'espace qu'occupe une douleur qu'on sent au milieu de la cuisse, il le faut parcourir avec la main : il n'en est pas de même si elle s'étend du genou à la hanche ; 
Devenu plus vif, il n'en donne point encore.

Rendons ce sentiment plus vif ; mais conservons-lui son uniformité ; échauffons, par exemple, l'air, ou refroidissons-le, elle aura de tout son corps une sensation égale de chaud, ou de froid ; et je ne vois pas qu'il en résulte autre chose, sinon qu'elle sentira plus vivement son existence. Car une seule sensation, quelque vive qu'elle soit, ne peut pas donner une idée d'étendue à un être, qui ne sachant pas qu'il est étendu lui-même, n’a pas appris à étendre cette sensation, en la rapportant aux différentes parties de son corps.

Par conséquent, si notre statue ne vivait que par une suite de sentiments uniformes, elle serait aussi bornée dans ses opérations et dans ses connaissances qu'elle l'a été avec le sens de l'odorat.

Il peut même n'en pas donner, quoique modifié.

Si je la frappe successivement à la tête et aux pieds, je modifie à diverses reprises son sentiment fondamental : mais ces modifications sont elles-mêmes uniformes. Aucune ne lui peut donc faire remarquer qu'elle est étendue. On demandera peut-être, si étant frappée tout-à-lafois à la tête et aux pieds, elle ne sentira pas que ces modifications sont distantes.

Lorsque je la touche, ou la sensation qu'elle éprouve, occupe si fort sa capacité de sentir, qu'elle attire l'attention toute entière ; ou l'attention continue encore de se porter au sentiment fondamental des autres parties. Dans le premier cas, notre statue ne saurait se représenter un intervalle entre sa tête et ses pieds ; car elle ne remarque point ce qui les sépare. Dans le second, elle ne le peut pas davantage ; puisque le sentiment fondamental ne donne aucune idée d'étendue.

parce que ce sont là deux points que nous savons être distants.

Ce n'est donc pas un sentiment uniforme qui nous donne l'idée de l'étendue de notre corps ; mais c'est la connaissance du volume de notre corps, qui nous fait attribuer de l'étendue à un sentiment uniforme.

Notre statue réduite au moindre degré de sentiment, n'a de tout son corps qu'un sentiment uniforme : elle ne sait donc pas qu'elle est étendue. 
Dans cet état la statue n'a point d'idée du mouvement.

J'agite son bras, et son moi reçoit une nouvelle modification ; acquerra-t-elle donc une idée de mouvement ? Non, sans doute ; car elle ne sait pas encore qu'elle a un bras, qu'il occupe un lieu, ni qu'il en peut changer. Ce qui lui arrive en ce moment, c'est de sentir plus particulièrement son existence dans la sensation que je lui donne, sans jamais pouvoir se rendre raison de ce qu'elle éprouve.

Il en sera de même, si je la transporte dans les airs. Tout alors se réduit en elle à une impression, qui modifie le sentiment fondamental tout entier ; et elle ne peut encore apprendre qu'elle a un corps qui se meut. 


\title{
Chapitre III
}

\author{
Des sensations qu'on attribue au toucher \\ et qui ne donnent cependant aucune idée d'étendue
}

\footnotetext{
La statue ne démêle les sensations qu'elle éprouve à-la-fois, qu'après les avoir remarquées successivement.
}

Que le sentiment de notre statue cesse d'être uniforme ; et modifions-le en même temps avec la même vivacité ; mais différemment dans toutes les parties de son corps ; il me paraît qu'elle n'aura point encore d'idée d'étendue. Ces sensations venant à-la-fois, il en résulte un sentiment confus, où la statue ne les saurait démêler ; parce que ne les ayant pas encore remarquées l'une après l'autre, elle n'a pas appris à en remarquer plusieurs ensemble.

Mais si la chaleur et le froid se font sentir successivement, elle les distinguera, et conservera une idée de chacun de ces sentiments. Qu'ensuite elle les éprouve ensemble, elle comparera l'impression qu'elle sent avec les idées que la mémoire lui rappelle ; et elle reconnaîtra qu'elle est tout-à-la-fois de deux manières différentes.

Nous pouvons également lui donner des idées de plusieurs autres espèces de plaisir et de douleur : car à mesure qu'elle apprendra à remarquer des sensations qui se succèdent, elle s'accoutumera à les remarquer, lorsqu'elles viennent plusieurs ensemble ; et elle parviendra même à en démêler au même instant un si grand nombre, qu'il ne lui sera pas possible de le déterminer.

Supposons, par exemple, qu'elle sente en même temps de la chaleur à un bras, du froid à l'autre, une douleur à la tête, un chatouillement aux pieds, un frémissement dans les entrailles, etc.; je crois qu'elle remarquera ces manières d'être, pourvu qu'elle les ait connues séparément, et qu'aucune ne dominant sur les autres, l'attention se 
partage également entre elles. Il faut appliquer ici les principes que nous avons établis en parlant de la vue.

Ces manières d'être, qu'elle remarque à-la-fois, co-existent, se distinguent plus ou moins, et sont à cet égard les unes hors des autres : mais parce qu'il n’en résulte ni contiguïté, ni continuité, elles ne sauraient donner à la statue aucune idée d'étendue : elles ne le peuvent pas plus des sons ou des odeurs. Si nous-mêmes nous nous les représentons comme étendues, ce n'est pas qu'elles donnent cette idée par elles-mêmes ; c'est que sachant d'ailleurs que nous avons un corps, nous les rapportons à une chose, dont les parties, les unes hors des autres et contiguës, forment un continu. Voilà donc les sensations qui appartiennent au toucher, et qui cependant ne sauraient produire le phénomène de l'étendue. 


\section{Chapitre IV}

\section{Considérations préliminaires à la solution de la question : Comment nous passons de nos sensations à la connaissance des corps}

\section{Comment nous concevons les corps.}

Nous ne saurions faire de l'étendue qu'avec de l'étendue, comme nous ne saurions faire des corps qu'avec des corps: car nous ne voyons pas qu'entre plusieurs choses inétendues, il puisse y avoir contiguïté, ni que par conséquent elles puissent former un continu. Cependant nous nous représentons nécessairement chaque corps, comme un continu formé par la contiguïté de plusieurs autres corps étendus. Nous sommes forcés de nous représenter ainsi jusqu'à ceux qui ne tombent pas sous les sens : nous les jugeons chacun composés d'autres corps étendus, ceux-ci d'autres encore, et nous ne savons plus où nous arrêter.

\section{Propriété des sensations qui nous en donne la} connaissance.

$\S 2$. Il est donc évident que nous ne passerons de nos sensations à la connaissance des corps, qu'autant qu'elles produiront le phénomène de l'étendue, et puisqu'un corps est un continu, formé par la contiguïté d'autres corps étendus, il faut que la sensation qui le représente, soit un continu formé par la contiguïté d'autres sensations étendues. Nous n’avons trouvé cette propriété dans aucune des sensations que nous avons observées : il nous reste à chercher si nous la trouverons dans d'autres. 
Moyen unique par lequel la nature nous conduit à cette connaissance.

$\S 3$. Les sensations n’appartenant qu'à l'âme, elles ne peuvent être que des manières d'être de cette substance. Elles sont concentrées en elle, elles ne s'étendent point au-delà. Or, si l'âme ne les apercevait que comme des manières d'être, qui sont concentrées en elle, elle ne verrait qu'elle dans ses sensations : il lui serait donc impossible de découvrir qu'elle a un corps, et qu'au-delà de ce corps il y en a d'autres.

Cependant cette découverte est une des premières qu'elle fait, et il ne fallait pas qu'elle tardât à la faire. Comment un enfant, qui vient de naître, s'occuperait-il de ses besoins, s'il n'avait aucune connaissance de son corps, et s'il ne se faisait pas, avec la même facilité, quelque idée des corps qui le peuvent soulager?

J'ai fait remarquer plusieurs fois, et particulièrement dans ma Logique, qu'il ne nous arrive jamais de faire une chose avec dessein, qu'autant que nous l'avons déjà faite, sans avoir eu le projet de la faire. C'est une vérité féconde, je ne dis pas un principe : car on a tant abusé de ce mot qu'on ne sait plus ce qu'il signifie.

Il résulte de cette vérité, que la nature commence tout en nous : aussi ai-je démontré que, dans le principe ou dans le commencement, nos connaissances sont uniquement son ouvrage, que nous ne nous instruisons que d'après ses leçons; et que tout l'art de raisonner consiste à continuer comme elle nous a fait commencer.

Or, la première découverte que fait un enfant, est celle de son corps. Ce n'est donc pas lui proprement qui la fait, c'est la nature qui la lui montre toute faite.

Mais la nature ne lui montrerait pas son corps, si elle ne lui faisait jamais apercevoir les sensations qu'il éprouve, que comme des modifications qui n'appartiennent qu'à son âme. Le moi d'un enfant, concentré alors dans son âme, ne pourrait jamais regarder les différentes parties de son corps comme autant de parties de lui-même.

La nature n’avait donc qu'un moyen de lui faire connaître son corps, et ce moyen était de lui faire apercevoir ses sensations non 
comme des manières d'être de son âme, mais comme des modifications des organes qui en sont autant de causes occasionnelles. Par-là le moi, au lieu d'être concentré dans l’âme, devait s'étendre, se répandre et se répéter en quelque sorte dans toutes les parties du corps.

Cet artifice, par lequel nous croyons nous trouver dans des organes qui ne sont pas nous proprement, a sans doute son fondement dans le mécanisme du corps humain, et sans doute aussi ce mécanisme aura été choisi et ordonné par rapport à la nature de l'âme. C'est tout ce que nous pouvons savoir à ce sujet. Quand on connaîtra parfaitement et la nature de l'âme et le mécanisme du corps humain, il est vraisemblable qu'on expliquera facilement, comment le moi, qui n'est que dans l'âme, paraît se trouver dans le corps. Quant à nous, il nous suffira d'observer ce fait et de nous en assurer.

Quoique la statue doive avoir des sensations qu'elle aperçoit naturellement comme des modifications de ses organes; cependant elle ne connaîtra pas son corps, aussitôt qu'elle éprouvera de pareilles sensations. Pour le découvrir, elle a besoin d'analyser, c'est-à-dire, qu'il faut qu'elle observe successivement son moi, dans toutes les parties où il paraît se trouver. Or il est certain qu'elle ne fera pas cette analyse toute seule : c'est donc à la nature à la lui faire faire. Observons. 


\section{Chapitre V}

\section{Comment un homme borné au toucher découvre son corps et apprend qu'il y a quelque chose hors de lui}

La statue a des mouvements.

Je donne à la statue l'usage de tous ses membres : mais quelle cause l'engagera à les mouvoir ? Ce ne peut pas être le dessein de s'en servir. Car elle ne sait pas encore qu'elle est composée de parties, qui peuvent se replier les unes sur les autres, ou se porter sur les objets extérieurs. C'est donc à la nature à commencer : c'est à elle à produire les premiers mouvements dans les membres de la statue.

\section{Comment ils sont produits.}

Si elle lui donne une sensation agréable, on conçoit que la statue en pourra jouir, en conservant toutes les parties de son corps dans la situation où elles se trouvent, et une pareille sensation paraît tendre à maintenir le repos plutôt qu’à produire le mouvement.

Mais s'il lui est naturel de se livrer à une sensation qui lui plaît et d'en jouir dans le repos, il lui est également naturel de se refuser à une sensation qui la blesse. Il est vrai qu'elle ne sait pas comment le peut se refuser à une pareille sensation ; mais dans les commencements, elle n'a pas besoin de le savoir, il lui suffit d'obéir à la nature. C'est une suite de son organisation, que ses muscles, que la douleur contracte, agitent ses membres, et qu'elle se meuve sans en avoir le dessein, sans savoir encore qu'elle se meut.

Il peut même y avoir aussi des sensations agréables, dont la vivacité ne lui permettra pas de rester dans un parfait repos ; au moins est-il certain que le passage alternatif du plaisir à la douleur et de la douleur au plaisir, doit occasionner des mouvements dans son corps. Si elle 
n'était pas organisée pour être mue à l'occasion des sensations agréables ou désagréables qu'elle éprouve, le repos parfait, auquel elle serait condamnée, ne lui laisserait aucun moyen pour rechercher ce qui peut lui être utile, et pour éviter ce qui lui peut nuire.

Mais dès que, par une suite de son organisation, il se fait en elle des mouvements, à l'occasion du plaisir, de la douleur, ou du passage alternatif de l'un à l'autre; il ne peut pas ne pas arriver que, dans le nombre de ces mouvements, quelques-uns n'écartent ou ne suspendent une sensation qui la blesse, et que quelques autres ne lui procurent une sensation qui lui plaît. Elle aura donc un intérêt à étudier ses mouvements, et par conséquent elle apprendra d'eux tout ce qu'elle en peut apprendre.

C'est naturellement, machinalement, par instinct et à son insu qu'elle se meut ; et il nous reste à expliquer comment elle découvrira, d'après ses propres mouvements, qu'elle a un corps et qu'au-delà il y en a d'autres.

Si nous considérons la multitude et la variété des impressions que les objets font sur la statue, nous jugerons que ses mouvements doivent naturellement se répéter et se varier. Or, dès qu'ils se répètent et se varient, il lui arrivera nécessairement de porter, à plusieurs reprises, ses mains sur elle-même et sur les objets qui l'approchent.

En les portant sur elle-même, elle ne découvrira qu'elle a un corps, que lorsqu'elle en distinguera les différentes parties, et qu'elle se reconnaîtra dans chacune pour le même être sentant ; et elle ne découvrira qu'il y a d'autres corps, que parce qu'elle ne se retrouvera pas dans ceux qu'elle touchera.

\section{Sensation par laquelle l'âme découvre qu'elle a un corps.}

Elle ne peut donc devoir cette découverte qu'à quelqu'une des sensations du toucher. Or, quelle est cette sensation ?

L’impénétrabilité est une propriété de tous les corps ; plusieurs ne sauraient occuper le même lieu : chacun exclut tous les autres du lieu qu'il occupe. 
Cette impénétrabilité n’est pas une sensation. Nous ne sentons pas proprement que les corps sont impénétrables: nous jugeons plutôt qu'ils le sont, et ce jugement est une conséquence des sensations qu'ils font sur nous.

La solidité est surtout la sensation d'où nous tirons cette conséquence; parce que, dans deux corps solides qui se pressent, nous apercevons, d'une manière plus sensible, la résistance qu'ils se font l'un à l'autre pour s'exclure mutuellement. S'ils pouvaient se pénétrer, les deux se confondraient dans un seul : mais dès qu'ils sont impénétrables, ils sont nécessairement distincts et toujours deux.

Il n'en est donc pas de la sensation de solidité, comme des sensations de son, de couleur et d'odeur, que l'âme qui ne connaît pas son corps, aperçoit naturellement comme des modifications où elle se trouve et ne trouve qu'elle. Puisque le propre de cette sensation est de représenter à-la-fois deux choses qui s'excluent l'une hors de l'autre, l'âme n'apercevra pas la solidité comme une de ces modifications où elle ne trouve qu'elle-même ; elle l'apercevra nécessairement comme une modification, où elle trouve deux choses qui s'excluent, et par conséquent elle l'apercevra dans ces deux choses.

Voilà donc une sensation par laquelle l'âme passe d'elle hors d'elle, et on commence à comprendre comment elle découvrira des corps.

En effet, puisque la statue est organisée pour avoir des mouvements, à la seule occasion des impressions qui se font sur elle, nous pouvons supposer que sa main se portera naturellement sur quelque partie de son corps, sur la poitrine, par exemple. Alors sa main et sa poitrine se distingueront à la sensation de solidité qu'elles se renvoient mutuellement, et qui les met nécessairement l'une hors de l'autre. Cependant en distinguant sa poitrine de sa main, la statue retrouvera son moi dans l'une et dans l'autre, parce qu'elle se sent également dans toutes deux. Quelqu'autre partie de son corps qu'elle touche, elle la distinguera de la même manière, et elle s’y retrouvera également.

Quoique cette découverte soit due principalement à la sensation de solidité, elle se fera plus facilement encore, s'il s’y joint d'autres sensations. Que la main soit froide, par exemple, et que la poitrine soit chaude, la statue les sentira comme quelque chose de solide et de froid 
qui touche quelque chose de solide et de chaud : elle apprendra à rapporter le froid à la main, la chaleur à la poitrine, et elle en distinguera mieux l'une de l'autre. Ainsi ces deux sensations, peu propres par elles-mêmes à faire connaître à la statue qu'elle a un corps, contribueront cependant à lui en donner une idée plus sensible, lorsqu'elles seront enveloppées dans la sensation de solidité.

Si jusqu'ici la main de la statue, en se portant d'une partie de son corps sur une autre, a toujours franchi des parties intermédiaires, elle se retrouvera dans chacune, comme dans autant de corps différents, et elle ne saura pas encore que, toutes ensemble, elles n'en forment qu'un seul. C'est que les sensations qu'elle a éprouvées, ne les lui représentent pas comme contiguës, ni par conséquent, comme formant un seul continu.

Mais s'il lui arrive de conduire sa main le long de son bras, et sans rien franchir, sur sa poitrine, sur sa tête, etc., elle sentira, pour ainsi dire, sous sa main, une continuité de moi ; et cette même main, qui réunira, dans un seul continu, les parties auparavant séparées, en rendra l'étendue plus sensible.

\section{A quoi elle reconnaît le sien.}

La statue apprend à connaître son corps, et à se reconnaître dans toutes les parties qui le composent ; parce qu'aussitôt qu'elle porte la main sur une d'elles, le même être sentant se répond en quelque sorte de l'une à l'autre : c'est moi. Qu'elle continue de se toucher, partout la sensation de solidité représentera deux choses qui s'excluent et qui en même temps sont contiguës, et partout aussi le même être sentant se répondra de l'une à l'autre : c'est moi, c'est moi encore ! Il se sent dans toutes les parties du corps. Ainsi il ne lui arrive plus de se confondre avec ses modifications, et de se multiplier comme elles : il n'est plus la chaleur et le froid, mais il sent la chaleur dans une partie, et le froid dans une autre.

\section{Comment elle découvre qu'il y en a d'autres.}

Tant que la statue ne porte les mains que sur elle-même, elle est à son égard, comme si elle était tout ce qui existe. Mais si elle touche un corps étranger, le moi, qui se sent modifié dans la main, ne se sent pas 
modifié dans ce corps. Si la main dit moi, elle ne reçoit pas la même réponse. La statue juge par là ses manières d'être tout-à-fait hors d'elle. Comme elle en a formé son corps, elle en forme tous les autres objets. La sensation de solidité, qui leur a donné de la consistance dans un cas, leur en donne aussi dans l'autre ; avec cette différence, que le moi, qui se répondait, cesse de se répondre.

\section{A quoi se réduit l'idée qu'elle a des corps.}

Elle n'aperçoit donc pas les corps en eux-mêmes ; elle n'aperçoit que ses propres sensations. Quand plusieurs sensations distinctes et co-existantes sont circonscrites par le toucher dans des bornes, où le moi se répond à lui-même, elle prend connaissance de son corps ; quand plusieurs sensations distinctes et co-existantes sont circonscrites par le toucher dans des bornes où le moi ne se répond pas, elle a l'idée d'un corps différent du sien. Dans le premier cas, ses sensations continuent d'être des qualités à elle ; dans le second, elles deviennent les qualités d'un objet tout différent.

Son étonnement de n'être pas tout ce qu'elle touche.

Lorsqu'elle vient d'apprendre qu'elle est quelque chose de solide, elle est, je m'imagine, bien étonnée de ne pas se trouver dans tout ce qu'elle touche. Elle étend les bras, comme pour se chercher hors d'elle; et elle ne peut encore juger si elle ne s'y retrouvera point : l'expérience pourra seule l'en instruire.

\section{Effet de cet étonnement.}

De cet étonnement, naît l'inquiétude de savoir où elle est, et, si j'ose m'exprimer ainsi, jusqu'où elle est. Elle prend donc, quitte et reprend tout ce qui est autour d'elle : elle se saisit, elle se compare avec les objets qu'elle touche; et à mesure qu'elle se fait des idées plus exactes, son corps, et les objets lui paraissent se former sous ses mains. 

A chaque chose qu'elle touche, elle croit
toucher tout.

Mais je conjecture qu'elle sera longtemps, avant d'imaginer quelque chose, au-delà des corps, que sa main rencontre. Il me semble, que lorsqu'elle commence à toucher, elle doit croire toucher tout ; et que ce ne sera qu'après avoir passé d'un lieu dans un autre, et avoir manié bien des objets, qu'elle pourra soupçonner qu'il y a des corps au-delà de ceux qu'elle saisit.

Comment elle a appris à toucher.

Mais comment apprend-elle à toucher ? C'est que des mouvements faits au hasard lui ayant procuré successivement des sensations agréables et désagréables, elle veut jouir des unes, et écarter les autres. Sans doute que dans les commencements elle ne connaît pas encore l'art de régler ses mouvements. Souvent même elle trouve ce qu'elle ne cherche pas, ou ce qu'il serait de son intérêt de fuir. Elle ne sait seulement pas comment elle doit conduire sa main pour la porter sur une partie de son corps, plutôt que sur une autre. Elle fait des essais, elle se méprend, elle réussit : elle remarque les mouvements qui l'ont trompée, et elle les évite ; elle remarque ceux qui ont répondu à ses désirs, et elle les répète. En un mot, elle tâtonne, et elle se fait peu-à-peu une habitude des mouvements qui la rendent capable de veiller à sa conservation. C'est alors qu'il y a dans son corps des mouvements qui correspondent aux désirs de son âme ; c'est alors que la statue se meut à sa volonté. 


\section{Chapitre VI}

\section{Du plaisir, de la douleur, des besoins, et des désirs dans un homme borné au sens du toucher}

La statue a du plaisir à démêler les différentes parties de son corps.

Donnons à notre statue l'usage de tous ses membres ; et avant de faire la recherche des connaissances qu'elle acquerra, voyons quels sont ses besoins.

Les différentes espèces de plaisir et de douleur en seront la source : car il faut raisonner sur le toucher, comme nous avons fait sur les autres sens.

D’abord son plaisir, ainsi que son existence, lui a paru concentré en un point. Mais ensuite il s'est peu-à-peu étendu avec le même progrès que le sentiment fondamental. Car elle a du plaisir à remarquer ce sentiment, lorsqu'il se démêle dans les parties de son corps ; pourvu qu'il ne soit accompagné d'aucune sensation douloureuse.

A se mouvoir.

Le plus grand bonheur des enfants paraît consister à se mouvoir : les chutes mêmes ne les dégoûtent pas. Un bandeau sur les yeux les chagrinerait moins qu'un lien, qui leur ôterait l'usage des pieds et des mains. En effet, c'est au mouvement qu'ils doivent la conscience la plus vive qu'ils aient de leur existence. La vue, l'ouïe, le goût, l'odorat semblent la borner dans un organe; mais le mouvement la répand dans toutes les parties, et fait jouir du corps dans toute son étendue.

Si l'exercice est pour eux le plaisir qui a le plus d'attrait, il en aura encore plus pour notre statue : car non seulement elle ne connaît rien 
qui puisse l'en distraire ; mais encore elle en éprouvera que le mouvement peut seul lui procurer tous les plaisirs dont elle est capable.

A manier les objets.

Elle aimera surtout les corps qui ne l'offensent point : elle sera fort sensible au poli et à la douceur de leur surface : et elle se plaira à y trouver au besoin de la fraîcheur ou de la chaleur.

Tantôt les objets lui feront plus de plaisir, à proportion qu'elle les maniera plus facilement : tels sont ceux qui par leur grandeur et leur figure s'accommoderont mieux à l'étendue et à la forme de sa main. D'autres fois ils lui plairont par l'étonnement où elle sera de leur volume, et par la difficulté de les manier. La surprise que lui donnera, par exemple, l'espace qu'elle découvrira autour d'elle, contribuera à lui rendre agréable le transport de son corps d'un lieu dans un autre.

La solidité et la fluidité, la dureté et la mollesse, le mouvement et le repos, seront pour elle des sentiments agréables: car plus ils contrastent, plus ils attirent son attention, et se font remarquer.

A s'en faire des idées.

Mais ce qui deviendra pour elle une source de plaisirs, c'est l'habitude qu'elle se fera de comparer et de juger. Alors elle ne touchera pas les objets pour le seul plaisir de les manier ; elle en voudra connaître les rapports, et elle passera par autant de sentiments agréables, qu'elle se formera d'idées nouvelles. En un mot, les plaisirs naîtront sous ses mains, sous ses pas. Ils augmenteront, ils se multiplieront, jusqu'à ce que ses forces soient excédées. Alors ils commenceront à être mêlés de fatigue ; peu-à-peu ils s'évanouiront ; enfin il ne lui restera plus que de la lassitude, et le repos deviendra son plus grand plaisir.

Elle est plus exposée à la douleur qu'avec les autres sens.

Quant à la douleur, elle y sera avec le sens du toucher plus fréquemment exposée qu'avec les autres ; souvent même elle en trouvera la vivacité bien supérieure à celle des plaisirs qu'elle connaît. Mais 
l'avantage dont elle jouit, c'est que le plaisir est à sa disposition, et que la douleur ne se fait sentir que par intervalles.

En quoi consistent ses désirs.

Avec les autres sens, son désir consistait principalement dans l'effort des facultés de l'âme, pour lui retracer une idée agréable le plus vivement qu'il était possible. Cette idée était la seule jouissance qu'elle pouvait par elle-même se procurer ; puisqu'il n'était pas en son pouvoir de se donner des sensations. Mais l'espèce de désir dont elle est capable avec le toucher, embrasse l'effort de toutes les parties du corps, qui tendent à se mouvoir, et qui vont, pour ainsi dire, chercher des sensations sur tous les objets palpables. Nous-mêmes, lorsque nous désirons vivement, nous sentons que nos désirs enveloppent cette double tendance des facultés de l'âme, et des facultés du corps. Dès-lors la jouissance ne se borne plus aux idées que l'imagination représente, elle s'étend au-dehors sur tous les objets qui sont à portée ; et les désirs, au lieu de concentrer notre statue dans ses manières d'être, comme il arrivait avec les autres sens, l'entraîne continuellement hors d'elle.

\section{Quel en est l'objet ?}

Par conséquent son amour, sa haine, sa volonté, son espérance, sa crainte n'ont plus ses propres manières d'être pour seul objet : ce sont les choses palpables qu'elle aime, qu'elle hait, qu'elle espère, qu'elle craint, qu'elle veut.

Elle n'est donc pas bornée à n'aimer qu'elle : mais son amour pour les corps, est un effet de celui qu'elle a pour elle-même: elle n'a d'autre dessein en les aimant, que la recherche du plaisir, ou la fuite de la douleur ; et c'est là ce qui va lui apprendre à se conduire dans l'espace qu'elle commence à découvrir. 


\section{Chapitre VII}

\section{De la manière dont un homme borné au sens du toucher, commence à découvrir l'espace}

Le plaisir règle les mouvements de la statue.

Puisque les désirs consistent dans l'effort que les parties du corps font de concert avec les facultés de l'âme, notre statue ne peut désirer une sensation, qu'au même instant elle ne se meuve pour chercher l'objet qui peut la lui procurer. Elle sera donc déterminée à se mouvoir, toutes les fois qu'elle se rappellera les sensations agréables, dont le mouvement lui a donné la jouissance.

D’abord elle s'agite sans but déterminé, et cette agitation est ellemême un sentiment dont elle jouit avec plaisir ; car elle en sent mieux son existence. Si sa main rencontre ensuite un objet, qui fasse sur elle une impression agréable de chaleur ou de fraîcheur ; aussitôt tous ses mouvements sont suspendus, et elle se livre toute entière à ce nouveau sentiment. Plus il lui paraît agréable, plus elle y fixe son attention ; elle voudrait même toucher de toutes les parties de son corps, l'objet qui l'occasionne : et ce désir reproduit en elle des mouvements, qui, au lieu de se faire au hasard, tendent tous à lui procurer la jouissance la plus complète.

Cependant cet objet perd son degré de chaleur ou de fraîcheur ; et la jouissance cesse d'en être agréable. Alors la statue se souvient des premiers mouvements qui lui ont plu, elle les désire ; et s'agitant une seconde fois, sans autre dessein que de s'agiter, elle change peu-à-peu de place, et touche de nouveaux corps.

Un des premiers objets de sa surprise, c'est sans doute l'espace qu'elle découvre à chaque instant autour d'elle. Il lui semble qu'elle le tire du sein de son être, que les objets ne s'étendent sous ses mains 
qu'aux dépens de son propre corps; et plus elle se compare avec l'espace qui l'environne, plus elle sent ses bornes se resserrer.

A chaque fois qu'elle découvre un nouvel espace, et touche de nouveaux objets, elle suspend ses mouvements, ou les règle, pour mieux jouir des sensations qui lui plaisent ; et elle recommence à se mouvoir pour le seul plaisir de se mouvoir, aussitôt qu'elle cesse de les trouver agréables.

Lorsque, par ce moyen, elle a découvert un certain espace, et qu'elle a éprouvé un certain nombre de sensations, elle se rappelle au moins confusément tout ce dont elle a joui. Se souvenant d'un côté qu'elle le doit à ses mouvements, sentant de l'autre que ses mouvements sont à sa disposition ; elle désire de parcourir encore cet espace, et de se procurer les mêmes sensations qu'elle a appris à connaître. Elle ne se meut donc plus pour le seul plaisir de se mouvoir.

Mais comme elle ne passe pas toujours par les mêmes endroits, elle éprouve de temps en temps des sentiments qui lui étaient tout-à-fait inconnus, à mesure qu'elle en fait l'expérience, elle juge que ses mouvements sont propres à lui procurer de nouveaux plaisirs, et cet espoir devient le principe qui la meut.

\section{Elle devient capable de curiosité.}

Elle commence donc à juger qu'il y a des découvertes à faire pour elle ; elle apprend que les mouvements, qui sont à sa disposition, lui donnent le moyen d'y réussir ; et elle devient capable de curiosité.

En effet, la curiosité n’est que le désir de quelque chose de nouveau ; et ce désir ne peut naître, que lorsqu'on a déjà fait des découvertes, et qu'on croit avoir des moyens pour en faire encore. Il est vrai qu'on peut se tromper sur les moyens. Devenu curieux par habitude, on s'occupe souvent à des recherches, où il est impossible de faire des progrès. Mais c'est une méprise, où l'on ne serait pas tombé, si dans d'autres occasions on n'avait pas eu des succès plus favorables.

Elle ne l'était pas avec les autres sens.

Il n'était peut-être pas impossible que, lorsque notre statue recevait successivement les autres sens, l'habitude de passer par des manières 
d'être toujours différentes, ne lui en fît soupçonner d'autres, dont elle pourrait encore jouir : mais ne sachant pas comment elles devaient lui arriver, et n'ayant aucun moyen pour en obtenir la jouissance, elle ne pouvait pas s'occuper à découvrir en elle une nouvelle manière d'être. Il était bien plus naturel qu'elle tournât tous ses désirs vers les sentiments agréables qu'elle connaissait. C'est pourquoi je ne lui ai point supposé de curiosité.

La curiosité est un des principaux motifs de ses actions.

On sent que la curiosité devient pour elle un besoin, qui la fera continuellement passer d'un lieu dans un autre. Ce sera souvent l'unique mobile de ses actions. Sur quoi il faut remarquer que je ne m'écarte point de ce que j'ai établi, lorsque j'ai dit que le plaisir et la douleur sont la seule cause du développement de ses facultés. Car elle n'est curieuse que dans l'espérance de se procurer les sentiments agréables, ou d'en éviter qui lui déplaisent. Ainsi ce nouveau principe est une conséquence du premier, et le confirme.

La douleur suspend le désir qu'elle a de se mouvoir.

Dans les commencements, elle ne fait que se traîner ; elle va ensuite sur ses pieds et sur ses mains ; et rencontrant enfin une élévation, elle est curieuse de découvrir ce qui est au-dessus d'elle, et elle se trouve, comme par hasard, sur ses pieds. Elle chancèle, elle marche, en s'appuyant sur tout ce qui est propre à la soutenir ; elle tombe, se heurte, et ressent de la douleur. Elle n'ose plus se soulever, elle n'ose presque plus changer de place: la crainte de la douleur balance l'espérance du plaisir. Si cependant elle n'a point encore été blessée par les corps sur lesquels elle a porté la main, elle continuera d'étendre les bras sans défiance : mais à la première piqûre, cette confiance l'abandonnera, et elle demeurera immobile.

\section{Ce désir renaît accompagné de crainte.}

Peu-à-peu sa douleur se dissipe, et le souvenir, qui lui en reste, trop faible pour contenir le désir de se mouvoir, est assez fort pour la faire mouvoir avec crainte. Ainsi il ne faut que disposer des objets qui l'environnent, et nous lui rendrons sa première sécurité par des plai- 
sirs capables d'effacer jusqu'au souvenir de sa douleur, ou nous renouvellerons sa défiance par des sentiments douloureux.

Si nous laissons les choses à leur cours naturel, les accidents pourront être si fréquents, que la défiance ne la quittera plus.

Circonstances où la crainte l'aurait entièrement étouffé.

Si même, au premier instant, nous l'avions placée dans un lieu, où elle n'eût pu se mouvoir, sans s'exposer à des douleurs vives, le mouvement aurait cessé d'être un plaisir pour elle ; elle fût demeurée immobile, et ne se fût jamais élevée à aucune connaissance des objets extérieurs.

Crainte qui donne occasion à une sorte d'industrie.

Mais si nous veillons sur elle, pour qu’elle n’éprouve que de légères douleurs, et que ces douleurs soient même encore assez rares ; alors elle désirera de se mouvoir, et ce désir sera seulement accompagné de temps en temps de quelque défiance de ses mouvements. Elle ne sera donc plus dans le cas de demeurer pour toujours immobile : si elle craint un changement de situation, elle le désire, toutes les fois qu'il peut la soulager, et elle obéit tour-à-tour à ces deux sentiments.

De là naîtra une sorte d'industrie, c'est-à-dire l'art de régler ses mouvements avec précaution, et de faire usage des objets, qu'elle découvrira pouvoir servir à prévenir les accidents auxquels elle est exposée. Le même hasard, qui lui fera saisir un bâton, lui apprendra peu-àpeu qu'il peut l'aider à se soutenir, à juger des corps, contre lesquels elle pourrait se heurter, et à connaître les endroits où elle peut porter le pied en toute assurance. 
Table des matières

\section{Chapitre VIII}

Des idées que peut acquérir un homme borné au sens du toucher

Le plaisir et la douleur également nécessaires

à l'instruction de la statue.

Sans le plaisir, notre statue n'aurait jamais la volonté de se mouvoir : sans la douleur, elle se transporterait avec sécurité, et périrait infailliblement. Il faut donc qu'elle soit toujours exposée à des sensations agréables ou désagréables. Voilà le principe et la règle de tous ses mouvements. Le plaisir l'attache aux objets, l'engage à leur donner toute l'attention dont elle est capable, et à s'en former des idées plus exactes. La douleur l'écarte de tout ce qui peut lui nuire, la rend encore plus sensible au plaisir, lui fait saisir les moyens d'en jouir sans danger, et lui donne des leçons d'industrie. En un mot, le plaisir et la douleur sont ses seuls maîtres.

Ils déterminent seuls le nombre et l'étendue de ses connaissances.

Le nombre des idées, qui peuvent venir par le tact, est infini : car il comprend tous les rapports des grandeurs, c'est-à-dire, une science que les plus grands mathématiciens n’épuiseront jamais. Il ne s’agit donc pas d'expliquer ici la génération des idées qu'on peut devoir au toucher : il suffit de découvrir celles que notre statue acquerra ellemême. Les observations que nous avons faites nous fournissent le principe qui doit nous conduire dans cette recherche : c'est qu'elle ne remarquera dans ses sensations que les idées, auxquelles le plaisir et la douleur lui feront prendre quelque intérêt. L’étendue de cet intérêt déterminera l'étendue de ses connaissances. 
Ordre dans lequel elle acquerra des idées.

Quant à l'ordre, dans lequel elle acquerra, il aura deux causes. L'une sera la rencontre fortuite des objets, l'autre la simplicité des rapports ; car elle n'aura des notions exactes de ceux qui supposent un certain nombre de comparaisons, qu'après avoir étudié ceux qui en demandent moins.

Il est possible de suivre les progrès que la seconde de ces causes pourra lui faire faire ; il n'en est pas de même de ceux qu'elle devra à la première. Mais c'est une chose assez inutile, et chacun peut faire à ce sujet les suppositions qu'il jugera à propos.

\section{Premières idées qu'elle acquiert.}

Ses idées sur la solidité, la dureté, la chaleur, etc. Ne sont point absolues ; c'est-à-dire, qu'elle ne juge qu'un corps est solide, dur, chaud, qu'autant qu'elle le compare avec d'autres, qui ne le sont pas au même degré, ou qui ont des qualités différentes. Si tous les objets étaient également solides, durs, chauds, etc. Elle aurait les sensations de solidité, de dureté et de chaleur, sans le remarquer ; elle confondrait tous les corps à cet égard. Mais parce qu'elle rencontre tour-à-tour de la solidité et de la fluidité, de la dureté et de la mollesse, de la chaleur et du froid ; elle donne son attention à ces différences, elle les compare, elle en juge, et ce sont autant d'idées, par où elle apprend à distinguer les corps. Plus elle exercera ses jugements à ce sujet, plus son tact acquerra de finesse ; et elle se rendra peu-à-peu capable de discerner dans une même qualité jusqu'aux nuances les plus légères. Voilà les idées, qui demandent le moins de comparaisons, et par conséquent les premières qu'elle aura occasion de remarquer.

\section{Sa curiosité en devient plus grande.}

Ces connaissances appliquent avec une nouvelle vivacité son attention sur les objets qu'elle touche, elles les lui font considérer sous tous les rapports qui la frappent sensiblement. Plus elle en découvre, plus elle se fait une habitude de juger qu'elle en découvrira encore, et la curiosité devient pour elle un besoin plus pressant. 


\section{Combien elle a d'activité.}

Ce besoin sera le principal ressort des progrès de son esprit. Cependant je n'entreprendrai pas d'en suivre tous les effets, parce que je craindrais de m'égarer dans trop de conjectures. J'observerai seulement que la curiosité doit être chez elle bien plus active que chez le commun des hommes. L'éducation l'étouffe souvent en nous, par le peu de soin qu'on prend à la satisfaire; et, dans l'âge où nous sommes abandonnés à nous-mêmes, la multitude des besoins la contraint, et ne nous permet pas de suivre tous les goûts qu'elle nous inspirerait. Mais dans la statue je ne vois rien qui ne tende à l'augmenter. Les sentiments agréables qu'elle éprouve souvent, et les sentiments désagréables auxquels elle est quelquefois exposée ${ }^{20}$, doivent l'intéresser vivement à pouvoir reconnaître, aux plus légères différences, les objets qui les produisent. Elle va donc se livrer à l'étude des corps.

La statue se fait des idées de figure.

Lorsqu'elle n'avait que le sens de la vue, nous avons observé que son œil apercevait des couleurs, sans pouvoir remarquer l'ensemble d'aucune figure, sans avoir même proprement aucune idée d'étendue. La main a au contraire cet avantage, qu'elle ne peut manier un objet, qu'elle ne remarque l'étendue et l'ensemble des parties, qui le composent. Il suffit pour cet effet, qu'elle en sente la solidité. En serrant un caillou, notre statue se fait l'idée d'un corps différent d'un bâton, qu'elle a touché dans toute sa longueur : elle sent dans un cube des angles, qu'elle ne peut trouver dans un globe : elle n'aperçoit pas la même direction dans un arc et dans un jonc bien droit. En un mot, elle distingue les choses solides, suivant la forme que chacune fait prendre à sa main ; et elle considère, comme formant un seul tout, les portions d'étendue, qu'elle ne peut séparer, ou qu'elle sépare difficilement. Elle acquiert donc les idées de ligne droite, de ligne courbe, et de plusieurs sortes de figures.

20 Je dis quelquefois, parce que si ces sentiments se répétaient trop souvent, ils éteindraient tout-à-fait sa curiosité. 
En comparant les qualités contraires.

Mais si les premiers corps, qu'elle a occasion de toucher, faisaient tous prendre la même forme à sa main, si elle ne rencontrait, par exemple, que des globes de même volume, elle se bornerait à remarquer que l'un serait rude, l'autre poli, l'un chaud, l'autre froid, et elle ne donnerait aucune attention à la forme, que sa main prendrait constamment. Ainsi elle toucherait des globes, sans jamais s'en faire aucune idée. Qu'elle manie au contraire tour-à-tour des globes, des cubes, et d'autres figures de diverses grandeurs, elle sera frappée de la différence des formes, que prennent ses mains. Alors elle commence à juger que toutes les figures ne se ressemblent pas. Sa curiosité la porte aussitôt à chercher tous les côtés, par où elle diffère, et elle s'en forme peu-à-peu des notions exactes. Pour acquérir l'idée d'une figure, il faut donc qu'elle en remarque plusieurs, qui au premier attouchement contrastent par quelque endroit d'une manière sensible : il faut qu'une première différence aperçue lui fasse naître le désir d'en apercevoir d'autres. Elle ne désire, par exemple, de connaître un cube, qu'après l'avoir comparé avec un globe, et avoir trouvé dans l'un des angles qu'elle ne trouve pas dans l'autre. En un mot, elle ne cherche de nouvelles idées dans ses sensations, qu'autant qu'elle est prévenue par les premières différences, qui s'offrent à elle, lorsqu'elle touche successivement plusieurs objets.

Comment on peut juger des idées qu'elle se

fait des corps.

La notion d'un corps est plus complexe, à proportion qu'elle rassemble en plus grand nombre les perceptions et les rapports, que le tact démêle. Pour connaître quelles idées notre statue se formera des objets sensibles; il faut donc observer dans quel ordre elle jugera de ces perceptions et de ces rapports, et comment elle en fera différentes collections.

Deux sortes de sensations qu'elle peut comparer.

Ou les sensations qu'elle comparera sont simples à son égard ; parce que ce sont des impressions uniformes, dans lesquelles elle ne saurait distinguer plusieurs perceptions ; telle est le chaud ou le froid : ou 
ce sont des sensations composées de plusieurs autres, qu'elle peut démêler ; telle est l'impression d'un corps, où il y a tout-à-la-fois solidité, chaleur, figure, etc.

Ses jugements sur les sensations simples.

Les sensations simples sont de même, ou de différente espèce : c'est par exemple de la chaleur et de la chaleur, ou de la chaleur et du froid. Les jugements qu'elle peut porter à leur occasion, sont bien bornés.

Si les sensations sont de même espèce, elle sent qu'elles sont distinctes et semblables ; elle sent encore si les degrés en sont les mêmes, ou différents. Cependant elle n'a pas de moyen pour les mesurer, et elle n'en juge que par des idées vagues de plus et de moins. Elle sent que la chaleur de sa main droite n'est pas la même que la chaleur de sa main gauche ; mais elle n'en connaît qu'imparfaitement les rapports.

Si les sensations sont d'espèces différentes, elle aperçoit seulement que l'une n'est pas l'autre ; elle juge que le chaud n'est pas le froid : mais dans les commencements elle ignore que ce sont deux sensations contraires ; et pour le découvrir, il faut qu'elle ait occasion de remarquer que le chaud et le froid ne peuvent pas se trouver en même temps dans le même corps, et que l'un détruit toujours l'autre. Ainsi ce jugement, le chaud et le froid sont des sensations contraires, ne lui est pas aussi naturel qu'il paraît l'être ; elle le doit à l'expérience.

Dans toutes ces occasions il est évident qu'il lui suffit de donner son attention à deux sensations, pour former tous les jugements qu'elle est capable de porter.

Ses jugements sur les sensations composées.

Quand deux objets font chacun une sensation composée, elle aperçoit d'abord que l'un n'est pas l'autre : c'est là son premier jugement.

Mais nous avons vu que l'attention diminue, à proportion du nombre des perceptions, entre lesquelles elle se partage. Elle ne peut donc embrasser toutes celles que produisent deux corps, qu'elle ne soit faible à l'égard de chacune. 
La statue ne se formera par conséquent les notions des deux objets qu'autant que le plaisir bornera successivement son attention aux différentes perceptions qu'elle en reçoit, et les lui fera remarquer chacune en particulier. Elle juge d'abord de leur chaleur, en ne les considérant qu'à cet égard: elle juge ensuite de leur grandeur, en ne les considérant que sous ce rapport : et parcourant de la sorte toutes les idées qu'elle y remarque, elle forme une suite de jugements, dont elle conserve le souvenir. De là résulte le jugement total, qu'elle porte de l'un et de l'autre, et qui réunit dans chacun les perceptions, qu'elle y a successivement observées. Elle analyse donc naturellement : et cela confirme ce que j'ai démontré dans ma logique, que nous apprenons l'analyse de la nature même.

Pour les uns et pour les autres l’opération de

l'esprit est la même.

Les jugements, qui lui donnent les notions composées de deux corps, ne sont donc qu'une répétition de ce qu'elle a fait sur les perceptions qu'elle regarde comme simples. C'est l'attention donnée d'abord à deux idées, ensuite à deux autres, et ainsi successivement à toutes celles qu'elle est capable d'y remarquer : et s'il en reste, dont elle n'a pas jugé, c'est qu'elle ne leur a point encore donné d'attention, c'est qu'elle ne les a pas remarquées.

Par conséquent, lorsqu'elle compare deux objets, qu'elle en juge, et qu'elle s'en forme des notions complexes; il n'y a point en elle d'autre opération, que lorsqu'elle juge de deux perceptions simples : car elle ne fait jamais que donner son attention.

\section{La statue devient capable de réflexion.}

Quand elle n'avait que l'odorat, elle conduisait son attention d'une idée à une autre, elle en remarquait la différence : mais elle ne faisait pas des collections, dont elle déterminât les rapports.

Avec la vue elle pouvait à la vérité distinguer plusieurs couleurs qu'elle éprouvait ensemble : mais elle ne remarquait pas qu'elles formassent des tous figurés. Elle sentait seulement qu'elle était tout-à-lafois de plusieurs manières. 
Ce n'est qu'avec le tact, que détachant ces modifications de son moi, et les jugeant hors d'elle, elle en fait des tous différemment combinés, où elle peut démêler une multitude de rapports.

L'attention dont elle est capable avec le toucher, produit donc des effets bien différents de l'attention, dont elle était capable avec les autres sens. Or, cette attention, qui combine les sensations, qui en fait au-dehors des tous, et qui réfléchissant, pour ainsi dire, d'un objet sur un autre, les compare sous différents rapports ; c'est ce que j'appelle réflexion. Ainsi l'on voit pourquoi notre statue, sans réflexion avec les autres sens, commence à réfléchir avec le toucher ${ }^{21}$.

\section{Ce qu'est un corps à son égard.}

Un corps qu'elle touche, n'est donc à son égard que les perceptions de grandeur, de solidité, de dureté, etc. Qu'elle juge réunies : c'est là tout ce que le tact lui découvre, et elle n'a pas besoin, pour former un pareil jugement, de donner à ces qualités un sujet, un soutien, ou, comme parlent les philosophes, un substratum. Il lui suffit de les sentir ensemble.

\section{De quelles qualités elle compose les objets}

Autant elle remarque de collections de cette espèce, autant elle distingue d'objets; et elle ne les compose pas seulement des idées de grandeur, de solidité, de dureté, elle y fait encore entrer la chaleur ou le froid, le plaisir ou la douleur, et en général tous les sentiments que le tact lui apprend à rapporter au-dehors. Ses propres sensations deviennent donc les qualités des objets. Si elles sont vives, telle qu'une chaleur violente, elle les juge en même temps dans sa main et dans le corps qu'elle touche. Si elles sont faibles, telle qu'une chaleur douce, elle ne les juge que dans ces corps. Ainsi elle peut bien quelquefois cesser de les regarder comme à elles : mais elle ne cessera plus de les attribuer aux objets qui les occasionnent. C'est une erreur, où les au-

La réflexion n'étant dans l'origine que l'attention même, on pourrait la concevoir de manière qu'elle aurait lieu avec chaque sens. Mais pour être d'accord sur les questions de cette espèce, il suffit de s'entendre. Je fais cette note pour prévenir les disputes de mots : inconvénient fort ordinaire en métaphysique, et contre lequel on ne saurait trop se tenir en garde. 
tres sens n'ont pu la faire tomber ; puisqu'elle n'apercevait jamais ses sensations, que comme son moi modifié différemment.

Elle se fait des idées abstraites.

Nous venons de voir que, pour rassembler dans les objets les qualités qui leur conviennent, elle a été obligée de les considérer chacune à part. Elle a donc fait des abstractions : car abstraire, c'est séparer une idée de plusieurs autres, qui entrent avec elle dans la composition d'un tout.

En ne donnant, par exemple, son attention qu'à la solidité d'un corps, elle sépare cette qualité des autres auxquelles elle n'a point d'égard. Elle fait de la même manière les idées abstraites de figure, de mouvement, etc. Et aussitôt chacune de ces notions se généralise, parce qu'elle remarque qu'il n'en est point qui ne convienne à plusieurs objets, ou qui ne se retrouve dans plusieurs collections.

On voit par là, et par ce que nous avons dit en traitant des autres sens, que les idées abstraites naissent nécessairement de l'usage que nous voulons faire de nos organes; que par conséquent elles ne sont pas aussi éloignées de l'intelligence des hommes qu'on paraît le croire ; et que leur génération n'est pas assez difficile à comprendre, pour supposer que nous ne puissions les tenir que de l'auteur de la nature.

\section{On n’en saurait déterminer le nombre}

Lorsque la statue était bornée aux autres sens, elle ne pouvait faire des abstractions que sur ses propres manières d'être : elle en séparait certains accessoires, communs à plusieurs ; elle en séparait, par exemple, le contentement ou le mécontentement qui les accompagnaient, et elle faisait par ce moyen les notions générales de manières d'être agréables, et de manières d'être désagréables.

Mais actuellement qu'elle s'est accoutumée à prendre ses sensations pour les qualités des objets sensibles, c'est-à-dire, pour des qualités, qui existent hors d'elle, et pour ainsi dire, par groupes ; elle peut les détacher chacune, des collections dont elles font partie, les considérer à part, et former des abstractions sans nombre. Mais n’ayant pas déterminé l'étendue de sa curiosité, nous n’entreprendrons pas de la suivre ici dans toutes ces opérations. 
Elle étend ses idées sur les nombres.

Sa curiosité ne la bornera pas à n'étudier que les objets qui l'environnent. Elle se touchera elle-même, et elle étudiera surtout la forme de cet organe, avec lequel elle manie les corps. Elle examinera ses doigts, lorsqu'ils s'écartent, se rapprochent, se plient ; frappée de la ressemblance, qu'elle commence à découvrir entre ses mains, elle sera curieuse d'en juger encore mieux ; elle observera ses doigts un à un, deux à deux, etc. ; par là elle multipliera ses notions abstraites sur les nombres, et pourra apprendre que sa main droite a autant de doigts que sa main gauche.

Qu'elle considère alors un corps, elle juge qu'il est un, comme un de ses doigts : qu'elle en considère deux, elle juge qu'ils sont deux, comme deux de ses doigts. Voilà donc ses doigts devenus les signes des nombres. Mais nous ne pouvons assurer, jusqu'où elle portera ces sortes d'idées. Il me suffit de prouver par ces détails, qu'elles sont toutes renfermées dans le toucher ; et que notre statue les y remarquera, suivant le besoin qu'elle aura de les acquérir.

Ses autres idées en sont plus distinctes.

Ayant étendu ses idées sur les nombres, elle sera plus en état de se rendre compte de ses notions abstraites. Elle pourra, par exemple, remarquer qu'elle forme sur un même objet, jusqu'à cinq ou six abstractions : ou, pour parler autrement, qu'elle y peut observer séparément, jusqu'à cinq ou six qualités différentes. Auparavant elle en apercevait seulement une multitude, qu'il ne lui était pas possible de déterminer : ce qui ne pouvait manquer d'y répandre de la confusion. Ses progrès sur les nombres contribueront donc à ceux de toutes ses autres connaissances.

Elle ne s'élève pas aux notions abstraites

d'être et de substance.

Mais quelle que soit la multitude des objets qu'elle découvre, quelque combinaisons qu'elle en fasse ; elle ne s'élèvera jamais aux notions abstraites d'être, de substance, d'essence, de nature, etc. ; ces sortes de fantômes ne sont palpables qu'au tact des philosophes. Dans l'habitude où elle est de juger que chaque corps est une collection de 
plusieurs qualités, il lui paraîtra tout naturel qu'elles existent réunies, et elle ne songera pas à chercher quel en peut être le lien ou le soutien. L'habitude nous tient souvent lieu de raison à nous-mêmes, et il faut convenir qu'elle vaut bien quelquefois les raisonnements des philosophes.

\section{Les philosophes à ce sujet, n'en savent pas} plus qu'elle.

Mais supposé que la statue fut curieuse de découvrir comment ces qualités existent dans chaque collection, elle serait portée comme nous, à imaginer quelque chose qui en est le sujet ; et si elle pouvait donner un nom à ce quelque chose, elle aurait une réponse toute prête aux questions des philosophes. Elle en saurait donc autant qu'eux; c'est-à-dire, qu'ils n'en savent pas plus qu'elle. En effet leurs définitions expliquées clairement n'apprennent à un enfant même, que ce que les sens lui ont appris.

Idées qu'elle se fait de la durée.

Parmi les notions abstraites qu'elle acquiert, il y en a deux, qui méritent quelques considérations particulières : ce sont celles de durée et d'espace.

Dans le vrai, elle ne connaît la durée que par la succession de ses idées. Mais elle pourra se la représenter si sensiblement, en imaginant le passé par un espace qu'elle a parcouru, et l'avenir pour un espace à parcourir, que le temps sera à son égard comme une ligne, suivant laquelle elle se meut. Cette manière d'en juger, lui paraîtra même si naturelle, qu'elle pourra bien tomber dans l'erreur de croire, qu'elle ne connaît la durée, qu'autant qu'elle réfléchit sur le mouvement d'un corps. Quand on a plusieurs moyens pour se représenter une chose, on est ordinairement porté à regarder comme le seul, celui qui est plus sensible. C'est une méprise, que les philosophes mêmes ont peine à éviter. Aussi Locke est-il le premier, qui ait démontré que nous ne connaissons la durée que par la succession de nos idées.

\section{De l'espace.}

Comme elle connaît la durée par la succession de ses idées, elle connaît l'espace par la co-existence de ses idées. Si le toucher ne lui 
transmettait pas à-la-fois plusieurs sensations qu'il distingue, qu'il rassemble, qu'il circonscrit dans de certaines limites, et dont, en un mot, il fait un corps, elle n'aurait l'idée d'aucune grandeur. Elle ne trouve donc cette idée que dans la co-existence de plusieurs sensations. Or, dès qu'elle connaît une grandeur, elle a de quoi en mesurer d'autre; elle a de quoi mesurer l'intervalle qui les sépare, celui qu'elles occupent ; en un mot, elle a l'idée de l'espace. Comme elle n'aurait donc aucune idée de durée, si elle ne se souvenait pas d'avoir eu successivement plusieurs sensations; elle n'aurait aucune idée d'étendue ni d'espace, si elle n’avait jamais plusieurs sensations à-lafois.

Partout où elle ne trouve point de résistance, elle juge qu'il n'y a rien, et elle se fait l'idée d'un espace vide. Cependant ce n'est pas une preuve pour qu'il existe un espace sans matière : elle n'a qu'à se mouvoir avec quelque vivacité, pour sentir au moins un fluide qui lui résiste.

De l’immensité.

D’abord elle n'imagine rien au-delà de l'espace qu'elle découvre autour d'elle; et en conséquence elle ne croit pas qu'il y en ait d'autre. Dans la suite l'expérience lui apprend peu-à-peu qu'il s'étend plus loin. Alors l'idée de celui qu'elle parcourt devient un modèle, d'après lequel elle imagine celui qu'elle n'a point encore parcouru, et lorsqu'elle a une fois imaginé un espace où elle ne s'est point transportée, elle en imagine plusieurs les uns hors des autres. Enfin ne concevant point de bornes, au-delà desquelles elle puisse cesser d'en imaginer ; elle est comme forcée d'en imaginer encore, et elle croit apercevoir l’immensité même.

\section{De l'éternité.}

Il en est de même de la durée. Au premier moment de son existence elle n'imagine rien ni avant ni après. Mais lorsqu'elle s'est fait une longue habitude des changements auxquels elle est destinée, le souvenir d'une succession d'idées est un modèle d'après lequel elle imagine une durée antérieure et une durée postérieure ; de sorte que ne trouvant point d'instant dans le passé ni dans l'avenir, au-delà duquel elle ne puisse pas en imaginer d'autres, il lui semble que sa pensée em- 
brasse toute l'éternité. Elle se croit même éternelle, car elle ne se rappelé pas qu'elle ait commencé, et elle ne soupçonne pas qu'elle doive finir.

Les deux dernières ne sont qu'une illusion de son imagination.

Cependant elle n’a dans le vrai, ni l’idée de l'éternité, ni celle de l'immensité. Si elle juge le contraire, c'est que son imagination lui fait illusion, en lui représentant comme l'éternité et l'immensité même, une durée et un espace vagues, dont elle ne peut fixer les bornes.

Les sensations sont des idées pour la statue.

A chaque découverte qu'elle fait, elle éprouve que le propre de chaque sensation est de lui faire prendre connaissance, ou de quelque sentiment qu'elle juge en elle, ou de quelque qualité qu'elle juge audehors : c'est-à-dire, que le propre de chaque sensation est pour elle ce que nous appelons idée; car toute impression qui donne une connaissance, est une idée.

En quoi elles diffèrent des idées intellectuelles.

Si elle considère ses sensations comme passées, elle ne les aperçoit plus que dans le souvenir qu'elle en conserve, et ce souvenir est encore une idée ; car il redonne ou rappelle une connaissance. J'appellerai ces sortes d'idées intellectuelles, ou simplement idées, pour les distinguer des autres, que je continuerai de nommer sensations. Une idée intellectuelle est donc le souvenir d'une sensation. L'idée intellectuelle de solidité, par exemple, est le souvenir d'avoir senti de la solidité dans un corps qu'on a touché ; l'idée intellectuelle de chaleur est le souvenir d'une certaine sensation qu'on a eue ; et l'idée intellectuelle de corps est le souvenir d'avoir remarqué dans une même collection de l'étendue, de la figure, de la dureté, etc.

Différence que la statue met entre ses idées et ses sensations.

Or, notre statue sent une différence entre éprouver actuellement des sensations, et se souvenir de les avoir eues. Elle les distingue donc de ce que j’appelle idée intellectuelle. 
Elle remarque qu'elle a de ces sortes d'idées, sans rien toucher, et qu'elle n'a des sensations qu'autant qu'elle touche. La raison qui lui a fait juger ses sensations dans les objets, ne peut lui faire porter le même jugement sur ses idées intellectuelles. Celles-ci lui paraissent donc comme si elle ne les avait qu'en elle-même.

Si les sensations sont la source de ses connaissances, les idées en deviennent le fond.

Par les sensations, elle ne connaît que les objets présents au tact, et c'est par les idées qu'elle connaît ceux qu'elle a touchés, et qu'elle ne touche plus. Elle ne juge même bien des objets qu'elle touche, qu'autant qu'elle les compare avec ceux qu'elle a touchés : et comme les sensations actuelles sont la source de ses connaissances, le souvenir de ses sensations passées ou les idées intellectuelles en sont tout le fond : c'est par leur secours que les nouvelles sensations se démêlent, et se développent toujours de plus en plus.

Sans les idées, elle jugerait mal des objets qu'elle touche.

En effet, lorsqu'elle touche un objet, elle ne jugerait point de sa grandeur, ni de ses degrés de dureté, de chaleur, etc., si elle ne se souvenait pas d'avoir manié d'autres grandeurs, où elle a trouvé d'autres degrés de dureté et de chaleur. Mais dès qu'elle s'en souvient, elle juge par comparaison cet objet plus ou moins grand, plus ou moins dur, plus ou moins chaud. C'est donc au souvenir ou à l'idée intellectuelle, qu'elle conserve de certaines grandeurs, de certains degrés de dureté et de chaleur, qu'elle juge des nouveaux objets qu'elle rencontre : c'est ce souvenir, qui lui faisant faire des comparaisons, lui fait remarquer les différentes idées ou connaissances, que les sensations actuelles lui transmettent.

Elle ne remarque pas que dans l'origine les idées et les sensations sont la même chose.

Cependant, puisque nous avons vu que le souvenir n'est qu'une manière de sentir, c'est une conséquence que les idées intellectuelles ne diffèrent pas essentiellement des sensations mêmes. Mais vraisemblablement notre statue n'est pas capable de faire cette réflexion. Tout ce qu'elle peut savoir, c'est qu'elle a des idées qui lui servent pour 
régler ses jugements, et qui ne sont pas des sensations. Supposez donc qu'elle eût occasion de réfléchir sur l'origine de ses connaissances, voici, je pense, comment elle raisonnerait.

Mauvais raisonnements qu'elle pourrait faire.

"Mes idées sont bien différentes de mes sensations, puisque les unes sont en moi, et les autres au contraire dans les objets. Or, connaître, c’est avoir des idées. Mes connaissances ne dépendent donc d'aucune sensation. D'ailleurs je ne juge des objets qui font sur moi des impressions différentes, que par la comparaison que j'en fais aux idées que j’ai déjà. J'ai donc des idées, avant d'avoir des sensations. Mais ces idées, me les suis-je données à moi-même ? Non sans doute : comment cela serait-il possible ? Pour se donner l'idée d'un triangle, ne faudrait-il pas déjà l'avoir ? Or, si je l'avais, je ne me la donne pas. Je suis donc un être, qui par moi-même, ai naturellement des idées : elles sont nées avec moi. »

Les idées étant le fond de toutes nos connaissances, elles constituent plus particulièrement ce que nous nommons l'être pensant : et quoique les sensations soient le principe de la pensée, et n’appartiennent dans le vrai qu'à l'âme, elles paraissent s'arrêter dans le corps, et être tout-à-fait inutiles à la génération des idées. Notre statue ne manquerait donc pas de tomber dans l'erreur des idées innées, si elle était capable, comme nous, de se perdre dans de vaines spéculations. Mais ce n'est pas la peine d'en faire un philosophe, pour lui apprendre à raisonner si mal ${ }^{22}$.

22 C'est d'après de pareils raisonnements qu'on a accordé des sensations à des animaux auxquels on a refusé des idées, et qu’on a cru que nos idées ne venaient point des sens. Les philosophes considérant l'homme lorsqu'il a déjà acquis beaucoup de connaissances, et voyant qu'alors il a des idées indépendamment des sensations actuelles, ils n’ont pas vu que ces idées n'étaient que le souvenir des sensations précédentes; ils ont conclu au contraire que les idées avoient toujours précédé les sensations. De-là plusieurs systèmes ; celui des idées innées, celui du P. Malebranche, et celui de quelques anciens, tel que Platon, qui croyaient que l'âme avait été douée de toutes sortes de connaissances avant son union avec le corps ; et que par conséquent ce que nous croyons apprendre n'est qu'une réminiscence de ce que nous avons su. 
Ces connaissances ne sont que pratiques ; et la lumière qui la conduit n'est qu'un instinct.

N’ayant pas déterminé jusqu’où elle portera sa curiosité, principal mobile des opérations de son âme ; je n'entreprends pas d'entrer dans un plus grand détail des connaissances, que la réflexion peut lui faire acquérir. Il suffit d'observer que tous les rapports des grandeurs étant renfermés dans les sensations du tact, elle les remarquera, lorsqu'elle sera intéressée à les connaître. Mon objet n'est pas d'expliquer la génération de toutes ses idées: je me borne à démontrer qu'elles lui viennent par les sens ; et que ce sont ses besoins, qui lui apprennent à les démêler.

Sa méthode, pour les acquérir, est d'observer successivement, l'une après l'autre, les qualités qu'elle attribue aux objets : elle analyse naturellement, mais elle n'a aucun langage. Or, une analyse, qui se fait sans signes, ne peut donner que des connaissances bien bornées ; elles sont nécessairement en petit nombre ; et parce qu'il n'a pas été possible d'y mettre de l'ordre, la collection en doit être fort confuse. Lors donc que je traite des idées qu'acquiert la statue, je ne prétends pas qu'elle ait des connaissances dont elle puisse se rendre un compte exact : elle n'a que des connaissances pratiques. Toute sa lumière est proprement un instinct, c'est-à-dire, une habitude de se conduire d'après des idées dont elle ne sait pas se rendre compte, habitude qui, étant une fois contractée, la guide sûrement, sans qu'elle ait besoin de se rappeler les jugements qui la lui ont fait prendre. En un mot, elle a acquis des idées. Mais dès qu'une fois ses idées lui ont appris à se conduire, elle n'y pense plus, et elle agit par habitude. Pour acquérir des connaissances de théorie, il faut nécessairement avoir un langage : car il faut classer et déterminer les idées, ce qui suppose des signes employés avec méthode. Voyez la première partie de ma grammaire, ou ma logique. 


\section{Chapitre IX}

\section{Observations propres à faciliter l'intelligence de ce qui sera dit en traitant de la vue}

\section{Objet de ce chapitre.}

Après les détails où nous venons d'entrer, ce chapitre paraîtra toutà-fait inutile ; et j'avoue qu'il le serait, s'il ne préparait pas le lecteur à se convaincre des observations que nous ferons sur la vue. La manière, dont les mains jugent des objets par le moyen d'un bâton, de deux, ou d'un plus grand nombre, ressemble si fort à la manière, dont les yeux en jugent, par le moyen des rayons, que depuis Descartes on explique communément l'un de ces problèmes par l'autre. Le premier sera l'objet de ce chapitre.

\section{Comment la statue peut juger des distances et}

des situations à l'aide d'un bâton.

La première fois que la statue saisit un bâton, elle n'a connaissance que de la partie qu'elle tient : c'est là qu'elle rapporte toutes les sensations qu'il fait sur elle.

Elle ne sait donc pas qu'il est étendu ; et par conséquent, elle ne peut pas juger de la distance des corps, sur lesquels elle le porte.

Ce bâton peut être incliné différemment, et dès-lors il fait sur sa main des impressions différentes. Mais ces impressions ne lui apprennent pas qu'il est incliné, tant qu'elle ignore qu'il est étendu. Elles ne sauraient donc encore lui découvrir les différentes situations des objets.

Pour juger par ce moyen des distances, il faut qu'elle l'ait touché dans toute sa longueur; et pour juger des situations par l'impression 
qu'elle en reçoit, il faut que pendant qu'elle le tient d'une main, elle en étudie de l'autre la direction.

Avec deux.

Tant qu'elle ne saura pas juger de la direction de deux bâtons, dont la longueur lui est connue, et qu'elle tient, l'un de la main droite, l'autre de la main gauche ; elle ne pourra pas découvrir s'ils se croisent quelque part, ni même si leurs extrémités s'éloignent, ou si elles se rapprochent. Elle croira souvent toucher deux corps, lorsqu'elle n'en touchera qu'un : elle croira en haut ce qui est en bas ; en bas ce qui est en haut. Mais dès qu'elle sera capable de remarquer les différentes directions, suivant la différence des impressions; alors elle connaîtra la situation des bâtons, et par là, elle jugera de celle des corps.

Ce jugement ne sera d'abord qu'un raisonnement fort lent. Elle se dira en quelque sorte: ces bâtons ne peuvent se croiser, que l'extrémité de celui que je tiens de la main droite ne soit à ma gauche ; et que l'extrémité de celui que je tiens de la main gauche, ne soit à ma droite. Par conséquent les corps qu'ils touchent, sont dans une situation contraire à celle de mes mains ; et je dois juger à droite ce que je sens de la main gauche, et à gauche, ce que je sens de la main droite. Dans la suite ce raisonnement lui deviendra si familier, et se fera si rapidement, qu'elle jugera de la situation des corps, sans paraître faire la moindre attention à celle de ses mains.

Elle rapporte sa sensation à l'extrémité opposée à celle qu'elle saisit.

Ce n'est plus à l'extrémité qui agit sur sa main, qu'elle rapporte les sensations qu'un bâton lui transmet; elle sent au contraire à l'extrémité opposée, la dureté ou la mollesse des corps, sur lesquels elle le porte ; et cette habitude lui fera distinguer des sensations, qu'elle ne distinguait pas auparavant.

Supposons qu'elle appuie la paume de la main sur trois joncs d'égale longueur, et réunis comme s'ils n'en formaient qu'un seul ; elle aura une sensation confuse, ou elle ne démêlera pas l'action de chaque jonc. Écartons ces joncs seulement par le bas : aussitôt elle 
aperçoit distinctement trois points de résistance, et par-là, elle discerne l’impression que chaque jonc fait sur elle.

Mais il faut bien remarquer qu'elle ne fait cette différence, que parce qu'elle a appris à juger de l'inclinaison par la sensation. Si elle n'avait pas fait les expériences nécessaires pour porter ce jugement, elle sentirait dans sa main un seul point de résistance, soit que les joncs fussent réunis par le bas, soit qu’ils fussent écartés.

Cette expérience confirme le sentiment que j’ai adopté sur la vue. Car ne se peut-il pas que, comme la main, l'œil ne confonde des sensations semblables, lorsqu'il ne les juge qu'en lui-même, et qu'il ne commence à en faire la différence, qu'autant qu'il s'accoutume à les rapporter au-dehors ? Il suffit de considérer que les rayons font sur lui l'effet que les joncs font sur la main.

\section{Elle se fait une espèce de géométrie.}

Pour déterminer l'intervalle que laissent entre elles les extrémités de deux bâtons qui se croisent, il suffit à un géomètre de déterminer la grandeur des angles, et celle des côtés.

La statue ne peut pas suivre une méthode, où il y ait autant de précision. Mais elle sait à-peu-près quelle est la grandeur des bâtons, combien ils sont inclinés, le point où ils se croisent ; et elle juge que les extrémités qui portent sur les objets, s'écartent, ou se rapprochent dans la même proportion que les extrémités qu'elle saisit. On imagine donc comment à force de tâtonner, elle se fera une espèce de géométrie, et jugera de la grandeur des corps à l'aide de deux bâtons.

Si elle avait quatre mains, elle pourrait par le même artifice, juger tout-à-la-fois de la hauteur et de la largeur d'un objet ; et si elle en avait un plus grand nombre, elle pourrait l'apercevoir sous une plus grande quantité de rapports. Il suffirait qu'elle contractât l'habitude de porter des jugements sur les impressions que lui transmettraient dix bâtons ou davantage.

C’est ainsi, que sans aucune connaissance de la géométrie, elle se conduirait, en tâtonnant, d'après les principes de cette science ; et, pour dire encore plus, c'est ainsi que dans le développement de nos facultés, il y a des principes qui nous échappent, au moment même 
qu'ils nous guident. Nous ne les remarquons pas, et cependant, nous ne faisons rien que par leur influence.

Aussi la connaissance des principes de la géométrie serait-elle tout-à-fait inutile à notre statue. Ce ne serait jamais qu'en tâtonnant, qu'elle en pourrait faire l'application aux bâtons, dont elle se sert. Or, dès qu'elle tâtonne, elle porte nécessairement les mêmes jugements, que si elle raisonnait d'après ces principes. Il aurait donc été superflu de lui supposer des idées innées sur les grandeurs et sur les situations : c'est assez qu'elle ait des mains. 


\section{Chapitre X}

Du repos, du sommeil, et du réveil dans un homme borné au sens du toucher

Le repos de la statue.

Le mouvement paraît à notre statue un état si naturel, et elle a une si grande curiosité de se transporter partout, et de tout manier, qu'elle ne prévoit pas sans doute l'inaction, où elle ne peut manquer de tomber. Mais peu-à-peu ses forces l'abandonnent ; et commençant à sentir de la lassitude, elle la combat quelque temps par le désir qu'elle a encore de se mouvoir ; enfin, le repos devient le plus pressant de ses besoins, elle sent que malgré elle, sa curiosité cède ; elle étend les bras, et reste immobile.

Son sommeil.

Cependant, l'activité de sa mémoire se conserve encore; il lui semble qu'elle ne vit plus que par le souvenir de ce qu'elle a été : mais la mémoire se repose à son tour; les idées qu'elle retrace, s'affaiblissent insensiblement, et paraissent se perdre dans un éloignement, d'où elles jettent à peine une lueur qui va s'éteindre. Enfin, toutes les facultés sont assoupies : et c'est pour la statue l'état de sommeil.

\section{Son réveil.}

Au bout de quelques heures le repos commence à lui rendre ses forces. Ses idées reviennent lentement, passent rapidement; et son âme suspendue entre le sommeil et la veille, se sent comme une vapeur légère, qui, d'un moment à l'autre, se dissipe et se reproduit. Cependant le mouvement renaît peu-à-peu dans toutes les parties de son corps, ses idées se fixent, ses habitudes se renouvellent, son âme lui est rendue toute entière, elle croit vivre pour la seconde fois. 
Ce réveil lui paraît délicieux. Elle porte les mains sur elle avec étonnement, elle les porte sur tout ce qui l'environne : charmée de se retrouver et de retrouver encore les objets qui lui sont familiers ; sa curiosité et tous ses désirs renaissent avec plus de vivacité. Elle s’y livre toute entière, se transporte de côté et d'autre, reconnaît ce qu'elle a déjà connu, et acquiert de nouvelles connaissances. Elle se fatigue donc pour la seconde fois ; et cédant à la lassitude, elle s'abandonne encore au sommeil.

Elle prévoit qu'elle repassera par ces états.

En passant à plusieurs reprises par ces différents états, elle se fera une habitude de les prévoir ; et ils lui deviendront si naturels, qu'elle s’endormira et se réveillera sans être étonnée.

A quoi elle les distingue.

C'est au souvenir d'avoir passé de l'un à l'autre, qu'elle les distingue. Elle a d'abord senti ses forces l'abandonner insensiblement : elle les a senties ensuite se renouveler tout-à-coup. Ce passage brusque d'une inaction totale à l'exercice de toutes ses facultés la frappe, la surprend, et par-là, lui paraît une seconde vie. Il suffit donc de l'opposition qui est entre l'instant de faiblesse, qui a immédiatement précédé le sommeil, et l'instant de force où elle se réveille, pour qu'elle se sente, comme si elle avait cessé d'être. Si elle avait repris l'usage de ses facultés par des degrés insensibles, elle n'eût rien pu remarquer de semblable.

\section{Elle ne se fait pas d'idée de l'état du sommeil.}

Cependant, elle ne se représente pas ce que ce peut être que l'état d'où elle sort au réveil. Elle ne juge point quelle en a été la durée, elle ne sait pas même s'il a duré. Car rien ne peut lui faire soupçonner qu'il y ait eu en elle ni au-dehors quelque succession. Elle n'a donc aucune notion de l'état de sommeil, et elle n'en distingue l'état de veille, que par la secousse que lui donnent toutes ses facultés, au moment que les forces lui sont rendues. 


\section{Chapitre XI}

\section{De la mémoire, de l'imagination et des songes dans un homme borné au sens du toucher}

Comment les idées se lient dans la mémoire de la statue.

Les sensations qui viennent par le tact sont de deux espèces : les unes sont l'étendue, la figure, l'espace, la solidité, la fluidité, la dureté, la mollesse, le mouvement, le repos ; les autres sont la chaleur et le froid, et différentes espèces de plaisirs et de douleurs. Les rapports de celles-ci sont naturellement indéterminés. Elles ne se conservent donc dans la mémoire, que parce que les organes les ont transmises à plusieurs reprises. Mais celles-là ont des rapports, qui se connaissent avec plus d'exactitude. Notre statue mesure le volume des corps avec ses mains ; elle mesure l'espace en se transportant d'un lieu dans un autre ; elle détermine les figures, lorsqu'elle en compte les côtés, et qu'elle en suit le contour ; elle juge à la résistance de la solidité, ou de la fluidité, de la dureté, ou de la mollesse ; enfin, elle saisit une différence sensible entre le mouvement et le repos, lorsqu'elle considère si un corps change ou ne change pas de situation par rapport à d'autres. Voilà donc de toutes les idées, celles qui se lient le plus fortement, et le plus facilement dans sa mémoire.

Elles se lient toutes à celles de l'étendue.

D'un côté, elle s'est fait une habitude de rapporter toutes ses sensations à l'étendue ; puisqu'elle les regarde comme les qualités des objets, qu'elle touche. Toutes ses idées ne sont que de l'étendue chaude ou froide, solide ou fluide, etc. ; par là celles dont les rapports sont les plus vagues, comme celles dont les rapports se déterminent le mieux, sont toutes liées à une même idée. En un mot, toutes ses sensations ne sont à son égard, que des modifications de l'étendue. 
Le souvenir en est plus fort et plus durable.

D'un autre côté, la sensation de l'étendue est telle, que notre statue ne la peut perdre que dans un sommeil profond. Lorsqu'elle est éveillée, elle sent toujours qu'elle est étendue ; car elle sent toutes les parties de son corps, qui pèsent sur le lieu où elles reposent, et qui le mesurent. Tant qu'elle est éveillée, elle ne peut donc pas avec le tact, comme avec les autres sens, être entièrement privée de toute espèce de sensations. Il lui en reste toujours une à laquelle toutes les autres sont liées ; et que je regarde, par cette raison, comme la base de toutes les idées dont elle conserve le souvenir. Tout prouve donc que la mémoire des idées qui viennent par le tact, doit être plus forte et durer beaucoup plus, que celle des idées qui viennent par les autres sens.

En quoi consiste l'imagination de la statue.

Les idées peuvent se retracer avec plus ou moins de vivacité. Lorsqu'elles se réveillent faiblement, la statue se souvient seulement d'avoir touché tel ou tel objet : mais lorsqu'elles se réveillent avec force, elle se souvient des objets, comme si elle les touchait encore. Or, j'ai appelé imagination cette mémoire vive, qui fait paraître présent ce qui est absent.

La réflexion se joint à l'imagination.

Si nous joignons à cette faculté la réflexion, ou cette opération qui combine les idées ; nous verrons comment la statue pourra se représenter dans un objet les qualités, qu'elle aura remarquées dans d'autres. Supposons qu'elle désire de jouir tout-à-la-fois de plusieurs qualités, qu'elle n’a point encore rencontrées ensemble ; elle les imaginera réunies, et son imagination lui procurera une jouissance, qu'elle ne pourrait pas obtenir par le tact.

Sens le plus étendu, dans lequel on peut prendre le mot imagination.

Voilà la signification la plus étendue qu'on donne au mot imagination : c'est de le considérer comme le nom d'une faculté, qui combine les qualités des objets, pour en faire des ensembles, dont la nature n'offre point de modèles. Par là, elle procure des jouissances, qui à certains égards l'emportent sur la réalité même : car elle ne manque 
pas de supposer dans les objets dont elle fait jouir, toutes les qualités qu’on désire y trouver.

Jouissance à laquelle le toucher et l'imagination concourent.

Mais la jouissance, par le toucher, peut se réunir à celle qui se fait par l'imagination ; et ce sera alors pour la statue, les plus grands plaisirs, dont elle puisse avoir connaissance. Lorsqu'elle touche un objet, rien n'empêche que l'imagination ne le lui représente quelquefois avec des qualités agréables qu'il n’a pas, et ne fasse disparaître celles par où il pourrait lui déplaire. Il suffira pour cela d'un désir vif d'y rencontrer les unes, et de n'y pas trouver les autres.

\section{Excès où l'imagination fait tomber la statue.}

L'imagination ne peut lui offrir tant d'attraits de la part des objets, qu'elle ne lui fasse souvent trouver du plaisir à se mouvoir, lors même que ses membres fatigués commencent à se refuser à ses désirs. Elle lui retrace même quelquefois ce plaisir avec tant de vivacité, qu'elle la distrait de la lassitude de ses organes. Alors, il n'y a qu'un excès de fatigue, qui puisse lui faire goûter le repos. Un état de peine et de douleur sera le fruit d'un désir, auquel elle s'est livrée avec trop peu de modération ; et lorsqu'elle en aura souvent fait l'épreuve, elle apprendra à se méfier des attraits du plaisir, et sera plus attentive à consulter ses forces.

\section{État de songe.}

Entre la veille et le sommeil profond, nous pouvons distinguer deux états mitoyens : l'un où la mémoire ne rappelle les idées que d'une manière fort légère ; l'autre où l'imagination les rappelle avec tant de vivacité, et en fait des combinaisons si sensibles, qu'on croit toucher les objets qu'on ne fait qu'imaginer.

Lorsque la statue s'est endormie dans un lieu, où elle a appris à se conduire sans danger ; elle peut imaginer qu'il est semé d'épines, de cailloux, qu'elle marche, et qu'à chaque pas, elle se déchire, tombe, se heurte, et ressent de la douleur. Quoiqu'étonnée de ce changement, elle n'en peut douter ; et son état est le même pour elle, que si elle était éveillée, et que ce lieu fût en effet tel qu’il lui paraît. 
Cause des songes et du désordre dans lequel ils retracent les idées.

Pour découvrir la cause de ce songe, il suffit de considérer, qu'avant le sommeil, elle avait les idées d'un lieu où elle pouvait se promener sans crainte ; celles d'épines, de cailloux, de déchirements, de chute, de douleur; enfin, celles d'un lieu, où elle avait fait l'épreuve de toutes ces choses. Or, qu'arrive-t-il dans le sommeil ? C'est que cette dernière idée ne se réveille point du tout. Celles d'épines, de cailloux, de déchirements, de chute, de douleur, et du lieu où elle n'a rien connu de semblable, se retracent avec la même vivacité, que si les objets étaient présents ; et se réunissant, il faut que la statue croie que ce lieu est devenu tel que son imagination le lui représente. Si elle se fût rappelé le lieu où elle s'est déchirée, où elle a fait des chutes, elle ne fût pas tombée dans cette erreur. Il ne se fait donc dans les songes des associations si bizarres et si contraires à la vérité, que parce que les idées qui rétabliraient l'ordre, se trouvent interceptées.

Il n’est pas étonnant, qu'alors les idées se reproduisent dans un désordre, qui rapproche et réunit celles qui sont les plus étrangères. Ainsi que le sommeil est le repos du corps, il est celui de la mémoire, de l'imagination et de toutes les facultés de l'âme ; et ce repos a différents degrés. Si ces facultés sont entièrement assoupies, le sommeil est profond. Si elle ne le sont que jusqu'à un certain point, la mémoire et l'imagination assez éveillées, pour rappeler certaines idées, ne le sont pas assez pour en rappeler d'autres : dès lors celles qui se présentent, forment les ensembles les plus extraordinaires.

\section{Sentiment de la statue au réveil.}

Je frappe la statue au milieu de son rêve, et je l'arrache au sommeil. Son premier sentiment est la crainte ; osant à peine se mouvoir, elle étend les bras avec méfiance ; et toute étonnée de ne point retrouver les objets, dont elle a cru recevoir des blessures, elle se soulève et hasarde de marcher. Peu-à-peu elle se rassure ; elle ne sait pas si elle se trompe actuellement, ou si elle s’est trompée le moment précédent. Sa confiance augmente, et elle oublie l'état où elle s'est trouvée en songe, pour jouir uniquement de celui où elle est au réveil. 
Son embarras sur l'état de songe et sur celui de veille.

Cependant le sommeil lui devient encore nécessaire. Elle s’y livre, elle a de nouveaux songes, et au réveil ils sont suivis du même étonnement.

En effet, ces illusions doivent lui paraître bien étranges. Elle ne saurait soupçonner qu'elles se sont offertes à elle dans le temps qu'elle dormait, puisqu'elle n'a aucune idée de la durée de son sommeil. Au contraire elle ne doute pas qu'elle ne fut éveillée : car veiller, pour elle, c'est toucher et réfléchir sur ce qu'elle touche. Ses songes ne lui paraissent donc pas des songes, et elle n'en doit avoir que plus d'inquiétude. Elle ne comprend pas pourquoi elle porte sur les mêmes objets des jugements si différents ; elle ne sait où est l'erreur; et elle passe tour-à-tour de la défiance que lui donnent ses songes, à la confiance que lui rend l'état de veille.

Pourquoi elle a des songes dont elle se souvient, et d'autres qu'elle a oubliés.

Il n'est pas possible qu'elle se souvienne de toutes les idées, qu'elle a eues, étant éveillée ; il doit en être de même de celles qu'elle a eues dans le sommeil.

Quant à la cause qui lui rappelle quelques-uns de ses songes, voici mes conjectures.

Si l'impression en a été vive, et s'ils ont offert les idées dans un désordre, qui contredise d'une manière frappante les jugements qui ont précédé le temps où elle s'est endormie, son étonnement en ce cas lie ces idées à la chaîne de ses connaissances. Au réveil le même étonnement qui subsiste encore, lui fait faire des efforts pour se les rappeler en détail, et elle se les rappelle. Elle n'en aura au contraire aucun souvenir, si l'intervalle du songe au réveil a été assez long, et rempli par un sommeil assez profond, pour effacer toute l'impression de l'étonnement où elle a été. Enfin, s'il ne lui reste que peu de surprise, quelquefois elle ne se rappellera qu'une partie de son rêve, d'autres fois elle se souviendra seulement d'avoir eu des idées fort extraordinaires. 
Ses songes ne se gravent donc dans sa mémoire, que parce qu'ils se lient à des jugements d'habitude qu'ils contredisent ; et c'est la surprise où elle est encore à son réveil, qui l'engage à se les rappeler. 


\section{Chapitre XII}

\section{Du principal organe du toucher}

La mobilité et la flexibilité des organes est

nécessaire pour acquérir des idées par le tact.

Les détails des chapitres précédents démontrent assez que la main est le principal organe du tact. C'est en effet celui qui s'accommode le mieux à toutes sortes de surfaces. La facilité d'étendre, de raccourcir, de plier, de séparer, de joindre les doigts, fait prendre à la main bien des formes différentes. Si cet organe n'était pas aussi mobile et aussi flexible, il faudrait beaucoup plus de temps à notre statue pour acquérir les idées des figures : et combien ne serait-elle pas bornée dans ses connaissances, si elle en était privée !

Si ses bras étaient, par exemple, terminés au poignet, elle pourrait découvrir qu'elle a un corps, et qu'il y en a d'autres hors d'elle : elle pourrait, en les embrassant, se faire quelque idée de leur grandeur et de leur forme ; mais elle ne jugerait qu'imparfaitement de la régularité ou de l’irrégularité de leurs figures.

Elle sera encore plus bornée, si nous ne laissons aucune articulation dans ses membres. Réduite au sentiment fondamental, elle se sentira comme dans un point, s’il est uniforme ; et s’il est varié, elle se sentira seulement de plusieurs manières à-la-fois.

Mais plus de mobilité et de flexibilité que nous

n'en avons, $y$ serait inutile, ou même contraire.

Les organes du toucher étant moins parfaits, moins propres à transmettre des idées, à proportion qu'ils sont moins mobiles et moins flexibles, n'en pourrait-on pas conclure que la main serait d'un plus grand secours, si elle était composée de vingt doigts, qui eussent cha- 
cun un grand nombre d'articulations ? Et si elle était divisée en une infinité de parties toutes également mobiles et flexibles, un pareil organe ne serait-il pas une espèce de géométrie universelle ${ }^{23}$ ?

Ce n'est pas assez que les parties de la main soient flexibles et mobiles, il faut encore que la statue puisse les remarquer les unes après les autres, et s'en faire des idées exactes. Quelle connaissance auraitelle des corps par le tact, si elle ne pouvait connaître qu'imparfaitement l'organe avec lequel elle les touche ? Et quelle idée se formerait-elle de cet organe, si le nombre des parties en était infini ? Elle appliquerait la main sur une infinité de petites surfaces. Mais qu'en résulterait-il ? Une sensation si composée, qu'elle n’y pourrait rien démêler. L'étude de ses mains serait trop étendue pour elle ; elle s'en servirait sans pouvoir jamais bien les connaître; et elle n'acquerrait que des notions confuses.

Je dis plus : vingt doigts ne lui seraient peut-être pas si commodes que cinq. Il fallait que l'organe, qui devait lui donner la connaissance des figures les plus composées, fût peu composé lui-même ; sans quoi, il lui eût été difficile de s'en former une notion distincte; et par conséquent, c'eût été un obstacle aux progrès de ses connaissances : en pareil cas, elle aurait eu besoin d'un organe plus simple, qui étant connu plus facilement, l'eût mis en état de se faire une idée du plus composé.

23 « Si la main, dit M. de Buffon, avait un plus grand nombre de parties, qu’elle fût, par exemple, divisée en vingt doigts, que ces doigts eussent un plus grand nombre d'articulations et de mouvements, il n'est pas douteux que le sentiment du toucher ne fût infiniment plus parfait dans cette conformation qu'il ne l'est ; parce que cette main pourrait alors s'appliquer beaucoup plus immédiatement et plus précisément sur les différentes surfaces des corps ; et si nous supposons qu'elle fût divisée en une infinité de parties, toutes mobiles et flexibles, et qui pussent toutes s'appliquer en même temps sur tous les points de la surface des corps, un pareil organe serait une espèce de géométrie universelle (si je puis m'exprimer ainsi), par laquelle nous aurions dans le moment même de l'attouchement, des idées exactes et précises de la figure de tous ces corps, et de la différence même infiniment petite de ces figures. » Histoire naturelle et générale, t. III, p. 359. 
Il ne manque donc rien à la statue à cet égard.

Je crois donc qu'elle n'a rien à désirer à cet égard. En effet, que manque-t-il à ses mains ? S'il y a des idées qu'elles ne lui donnent pas immédiatement, elles la mettent sur la voie pour les acquérir. Quand on supposerait, ce qui n'est pas possible, qu'ayant un grand nombre de doigts très fins et très déliés, elle démêlerait toutes les impressions qu'ils lui transmettraient à-la-fois, elle n'en connaîtrait pas mieux les grandeurs, qui sont l'objet des mathématiques. Elle remarquerait seulement sur la surface des corps des inégalités, qui lui échappent aujourd'hui ; mais qui ne lui échapperont plus, lorsqu'elle jouira du sens de la vue. 


\section{TROISIÈME PARTIE}

\section{Comment le toucher apprend aux autres sens \\ à juger des objets extérieurs}

Table des matières

\section{Chapitre premier}

Du toucher avec l'odorat

Jugements de la statue sur les odeurs.

Joignons l'odorat au toucher, et rendant à notre statue le souvenir des jugements qu'elle a portés, lorsqu'elle était bornée au premier de ces sens, conduisons-la dans un parterre semé de fleurs ; aussitôt toutes ses habitudes se renouvellent, et elle se croit toutes les odeurs qu'elle sent.

Elle n'imagine pas quelle peut être la cause de ces sensations.

Étonnée de se trouver ce qu'elle a cessé d'être depuis si longtemps, elle n'en saurait encore soupçonner la cause. Elle ignore qu'elle vient de recevoir un nouvel organe ; et si le tact lui a appris qu'il y a des objets palpables, il ne lui apprend pas encore qu'aucun d'eux soit le principe des sentiments que nous venons de lui rendre.

Elle en juge au contraire d'après l'habitude où elle a été de les regarder comme des manières d'être, qu'elle ne doit qu'à elle-même. Il lui paraît tout naturel d'être tantôt une odeur, tantôt une autre : elle 
n'imagine pas que les corps y puissent contribuer : elle ne leur connaît que les qualités, que le tact seul y fait découvrir.

Elle est deux êtres différents.

La voilà tout-à-la-fois deux êtres bien différents : l'un, qu'elle ne peut saisir, et qui paraît lui échapper à chaque instant ; l'autre, qu'elle touche, et qu'elle peut toujours retrouver.

Elle commence à soupçonner que les odeurs

lui viennent des corps.

Portant au hasard la main sur les objets qu'elle rencontre, elle saisit une fleur qui lui reste dans les doigts. Son bras, mû sans dessein, l'approche et l'éloigne tour-à-tour de son visage : elle se sent d'une certaine manière, avec plus ou moins de vivacité.

Étonnée, elle répète cette expérience avec dessein. Elle prend et quitte plusieurs fois cette fleur. Elle se confirme qu'elle est, ou cesse d'être d'une certaine manière, suivant qu'elle l'approche ou l'éloigne. Enfin, elle commence à soupçonner qu'elle lui doit le sentiment dont elle est modifiée.

Elle découvre en elle l'organe de l'odorat.

Elle donne toute son attention à ce sentiment, elle observe avec quelle vivacité il augmente, elle en suit les degrés, les compare avec les différents points de distance, où la fleur est de son visage ; et l'organe de l'odorat ayant été plus affecté, lorsqu'il a été touché par le corps odoriférant, elle découvre en elle un nouveau sens.

Elle juge les odeurs dans les corps.

Elle recommence ces expériences: elle approche la fleur de ce nouvel organe, elle l'en éloigne : elle compare la fleur présente avec le sentiment produit, la fleur absente avec le sentiment éteint : elle se confirme qu'il lui vient de la fleur, elle juge qu'il y est.

Elle les sent dans les corps.

A force de répéter ce jugement, elle s'en fait une si grande habitude, qu'elle le porte au même instant qu'elle sent. Dès-lors, il se 
confond si bien avec la sensation, qu'elle n'en saurait faire la différence. Elle ne se borne plus à juger l'odeur dans la fleur, elle l'y sent.

Les odeurs deviennent les qualités des corps.

Elle se fait une habitude des mêmes jugements, à l'occasion de tous les objets qui lui donnent des sentiments de cette espèce ; et les odeurs ne sont plus ses propres modifications : ce sont des impressions que les corps odoriférants font sur l'organe de l'odorat ; ou plutôt ce sont les qualités mêmes de ces corps.

Combien elle a de peine à se familiariser avec

ces jugements.

Ce n'est pas sans surprise, qu'elle se voit engagée à porter des jugements aussi différents de ceux qui lui ont été auparavant si naturels ; et ce n'est qu'après des expériences souvent réitérées, que le toucher détruit les habitudes contractées avec l'odorat. Elle a autant de peine à mettre les odeurs au nombre des qualités des objets, que nous en avons nous-mêmes à les regarder comme nos propres modifications.

Elle distingue deux espèces de corps.

Mais enfin familiarisée peu-à-peu avec ces sortes de jugements, elle distingue les corps auxquels elle juge que les odeurs appartiennent, de ceux auxquels elle juge qu'elles n'appartiennent pas. Ainsi l'odorat, réuni au toucher, lui fait découvrir une nouvelle classe d'objets palpables.

Et plusieurs espèces de corps odoriférants.

Remarquant ensuite la même odeur dans plusieurs fleurs, elle ne la regarde plus comme une idée particulière ; elle la regarde au contraire comme une qualité commune à plusieurs corps. Elle distingue par conséquent autant de classes de corps odoriférants, qu'elle découvre d'odeurs différentes ; et elle se forme une plus grande quantité de notions abstraites ou générales, que lorsqu'elle était bornée au sens de l'odorat. 
Discernement qu'acquiert le sens de l'odorat.

Curieuse d'étudier de plus en plus ces nouvelles idées, tantôt elle sent les fleurs une à une, tantôt elle en sent plusieurs ensemble. Elle remarque la sensation qu'elles font séparément, et celle qu'elles font après leur réunion. Elle distingue plusieurs odeurs dans un bouquet, et son odorat acquiert un discernement qu'il n'eût point eu, sans le secours du tact.

Mais ce discernement aura des bornes, si les odeurs lui viennent d'une certaine distance, si elles sont en grand nombre, et si surtout le mélange en est tel, qu'elles ne dominent point les unes sur les autres ; elles se confondront dans l'impression qu'elles feront ensemble, et il lui sera impossible d'en reconnaître aucune. Cependant il y a lieu de conjecturer que son discernement à cet égard sera plus étendu que le nôtre : car les odeurs ayant plus d'attrait pour elle que pour nous, qui sommes partagés entre toutes les jouissances des autres sens, elle s’exercera davantage à en démêler les différences.

Ces deux sens, par l'exercice qu'ils se procurent mutuellement, produisent donc, étant réunis, des connaissances et des plaisirs qu'ils ne donnaient pas étant séparés.

$\begin{aligned} & \text { Jugements qui se confondent avec les } \\ & \text { sensations. }\end{aligned}$
.

Pour apercevoir sensiblement comment les jugements se distinguent des sensations, ou s'y confondent, parfumons des corps dont la figure peu composée soit familière à notre statue, et présentons-les-lui au premier moment que nous lui donnons le sens de l'odorat. Qu'une certaine odeur soit, par exemple, toujours dans un triangle, une autre dans un carré ; chacune se liera avec la figure qui lui est particulière ; et dès-lors, la statue ne pourra plus être frappée de l'une ou de l'autre, qu'aussitôt elle ne se représente un triangle ou un carré : elle croira sentir une figure dans une odeur, et toucher une odeur dans une figure.

Elle remarquera que s'il y a des figures qui n'ont point d'odeur, il n'y a point d'odeur qui n'emporte constamment une certaine figure ; et elle attribuera à l'odorat des idées qui n'appartiennent qu'au toucher. Pour bouleverser ensuite toutes ses notions, il n’y aurait qu'à 
parfumer de différentes odeurs des corps de même figure, et à parfumer de la même odeur des corps de figure différente.

\section{Jugements qui ne s'y confondent pas.}

Le jugement qui lie une figure triangulaire à une odeur, peut se répéter rapidement, toutes les fois que l'occasion s'en présente ; parce qu'il n'a pour objet que des idées peu composées. C’est pourquoi il est propre à se confondre avec la sensation. Mais si la figure était compliquée, il faudrait un plus grand nombre de jugements pour la lier à l'odeur. La statue ne se la représenterait plus avec la même facilité ; elle ne jugerait plus que la figure et l'odeur lui sont connues par le même sens.

Lorsqu'elle étudie, par exemple, une rose au toucher, elle lie l'odeur à l'ensemble des feuilles, à leur tissu, et à toutes les qualités par où le tact la distingue des autres fleurs qui lui sont connues. Par là, elle s'en fait une notion complexe, qui suppose autant de jugements, qu'elle y remarque de qualités propres à la lui faire reconnaître. A la vérité elle en jugera quelquefois à la première impression qu'elle sentira, en y portant la main. Mais elle y sera si souvent trompée, qu'elle s'apercevra bientôt que, pour éviter toute méprise, elle est obligée de se rappeler l'idée la plus distincte que le tact lui en a donnée; et de se dire, la rose diffère de l'œillet, parce qu'elle a telle forme, tel tissu, etc. Or, ces jugements étant en grand nombre, il ne lui est plus possible de les répéter tous, au moment qu'elle sent cette fleur. Au lieu donc de sentir les qualités palpables dans l'odeur, elle s'aperçoit qu'elle se les rappelle peu-à-peu ; et elle ne tombe plus dans l'erreur d'attribuer à l'odorat des idées qu'elle ne doit qu'au toucher.

Ses méprises sont fort sensibles, lorsqu'à l'occasion des odeurs, elle répète, sans le remarquer, des jugements dont elle a contracté l'habitude. Elle en fera qui le seront beaucoup moins, quand nous lui donnerons le sens de la vue. 


\section{Chapitre II}

\section{De l'ouïe, de l'odorat et du tact réunis}

État de la statue, au moment où nous lui rendons l'ouïe.

Notre statue sera, comme dans le chapitre précédent, étonnée de se trouver ce qu'elle a été, si au moment que nous ajoutons l'ouïe à l'odorat et au toucher, elle reprend toutes les habitudes qu'elle a contractées avec le premier de ces sens. Ici elle est le chant des oiseaux, là, le bruit d'une cascade, plus loin, celui des arbres agités, un moment après, le bruit du tonnerre ou d'un orage terrible.

Toute entière à ces sentiments, son tact et son odorat n'ont plus d'exercice. Qu'un silence profond succède tout-à-coup, il lui semblera qu'elle est enlevée à elle-même. Elle est quelque temps sans pouvoir reprendre l'usage de ses premiers sens. Enfin rendue peu-à-peu à elle, elle recommence à s’occuper des objets palpables et odoriférants.

Elle découvre en elle l'organe de l'ouïe.

Elle trouve ce qu'elle ne cherchait pas : car ayant saisi un corps sonore, elle l'agite sans en avoir le dessein ; et l'ayant par hasard tour-àtour approché et éloigné de son oreille, c'en est assez pour la déterminer à le rapprocher et à l'éloigner à plusieurs reprises. Guidée par les différents degrés d'impression, elle l'applique à l'organe de l'ouïe ; et après avoir répété cette expérience, elle juge les sons dans cette partie, comme elle a jugé les odeurs dans une autre.

Elle juge les sons dans les corps.

Cependant, elle observe que son oreille n'est modifiée qu'à l'occasion de ce corps : elle entend des sons, lorsqu'elle l'agite, elle 
n'entend plus rien, lorsqu'elle cesse de l'agiter. Elle juge donc que ces sons viennent de lui.

Elle les y entend.

Elle répète ce jugement, et elle parvient à le faire avec tant de promptitude, qu'elle ne remarque plus d'intervalle entre le moment où ces sons lui frappent l'oreille, et celui où elle juge qu'ils sont dans ce corps. Entendre ces sons et les juger hors d'elle, sont deux opérations qu'elle ne distingue plus. Au lieu donc de les apercevoir comme des manières d'être d'elle-même, elle les aperçoit comme des manières d'être du corps sonore. En un mot, elle les entend dans ce corps.

Elle se fait une habitude de cette manière d'entendre.

Si nous lui faisons faire la même expérience sur d'autres sons, elle portera encore les mêmes jugements, et elle les confondra avec la sensation. Dans la suite cette manière de sentir lui deviendra même si familière, que son oreille n'aura plus besoin des leçons du tact. Tout son lui paraîtra venir de dehors, même dans les occasions où elle ne pourra pas toucher les corps qui le transmettent. Car un jugement ayant été confondu par habitude avec une sensation, il doit se confondre avec toutes les sensations de même espèce.

\section{Discernement de son oreille.}

Si plusieurs sons que la statue a étudiés, raisonnent ensemble, elle les discernera, non-seulement parce que son oreille est capable d'en saisir jusqu'à un certain point la différence ; mais surtout, parce qu'elle vient de contracter l'habitude de les juger dans les corps qu'elle distingue. C'est ainsi que le toucher contribue à augmenter le discernement de l'ouïe.

Par conséquent, plus elle s’aidera du toucher pour faire la différence des sons, plus elle apprendra à les distinguer. Mais elle les confondra toutes les fois que les corps qui les produisent, cesseront de se démêler au tact. 
Le discernement de l'ouïe a donc des bornes, parce qu'il y a des cas où le toucher lui-même ne saurait tout démêler. Je ne parle pas des bornes qui ont pour cause un défaut de conformation.

\section{Elle juge à l'ouïe des distances et des situations.}

C'est sur les objets qui sont à la portée de sa main, que la statue commence à faire des expériences. En conséquence il lui semble d'abord, à chaque bruit qui frappe son oreille, qu'elle n'a qu'à étendre le bras pour saisir le corps qui le rend : car elle n'a pas encore appris à le juger plus éloigné. Mais comme elle y est trompée, elle fait un pas, elle en fait un second ; et à mesure qu'elle avance, elle observe que le bruit augmente, jusqu'au moment où le corps qui le produit, est aussi près d'elle qu'il peut l'être.

Ces expériences lui apprennent peu-à-peu à juger des différents éloignements de ce corps ; et ces jugements, devenus familiers, se répètent si rapidement, que se confondant avec la sensation même, elle reconnaît enfin les distances à l'ouïe. Elle apprendra de la même manière, si un corps est à sa droite ou à sa gauche. En un mot elle apercevra la distance et la situation d'un objet à l'ouïe, toutes les fois que l'une et l'autre seront les mêmes, que dans les cas, où elle a eu occasion de faire beaucoup d'expériences. N'ayant même que ce moyen pour s'en assurer, au défaut du tact, elle en fera si souvent usage, qu'elle jugera quelquefois aussi sûrement, que nous jugeons nousmêmes avec les yeux.

Mais elle courra risque de s'y méprendre, toutes les fois qu'elle entendra des corps dont elle n'aura pas encore étudié la variété des sons, suivant la variété des situations et des distances. Il faut donc qu'elle s'accoutume à porter autant de jugements différents, qu'il y a d'espèces de corps sonores et de circonstances où ils se font entendre.

\section{Erreurs où l'on pourrait la faire tomber.}

Si elle n’avait jamais entendu le même son, qu'elle n’eût touché la même figure et réciproquement ; elle croirait que les figures emportent avec elles les idées des sons, et que les sons emportent avec eux les idées des figures ; et elle ne saurait répartir au toucher et à l'ouïe les idées qui appartiennent à chacun de ces sens. De même si chaque 
son eût constamment été accompagné d'une certaine odeur, et chaque odeur d'un certain son ; il ne lui serait pas possible de distinguer les idées qu'elle doit à l'odorat, de celles qu'elle doit à l'ouïe. Ces erreurs sont semblables à celles où nous l'avons fait tomber dans le chapitre précédent; et elles préparent aux observations que nous allons faire sur la vue. 


\section{Chapitre III}

\section{Comment l'œil apprend à voir la distance, la situation, la figure, la grandeur et le mouvement des corps}

État de la statue, lorsque la vue lui est rendue.

L'étonnement de notre statue est encore la première chose à remarquer, au moment que nous lui rendons la vue. Mais il est vraisemblable que les expériences qu'elle a faites sur les sensations de l'odorat, de l'ouïe et du toucher, lui feront bientôt soupçonner que ce qui lui paraît encore des manières d'être d'elle-même, pourrait être des qualités qu'un nouveau sens va lui faire découvrir dans les corps.

\section{Pourquoi l'œil ne peut être instruit que par le}

toucher.

Nous avons vu qu'étant bornée au tact, elle ne pouvait pas juger des grandeurs, des situations et des distances, par le moyen de deux bâtons, dont elle ne connaissait ni la longueur ni la direction. Or, les rayons sont à ses yeux, ce que les bâtons sont à ses mains; et l'œil peut être regardé comme un organe, qui a en quelque sorte une infinité de mains, pour saisir une infinité de bâtons. S’il était capable de connaître par lui-même la longueur et la direction des rayons, il pourrait, comme la main, rapporter à une extrémité ce qu'il sentirait à l'autre ; et juger des grandeurs, des distances et des situations. Mais bien loin que le sentiment qu'il éprouve, lui apprenne la longueur et la direction des rayons ; il ne lui apprend pas seulement s’il y en a. L'œil n'en sent l'impression, que comme la main sent celle du premier bâton qu'elle touche par l'un des bouts.

Quand même nous accorderions à notre statue une connaissance parfaite de l'optique, elle n'en serait pas plus avancée. Elle saurait qu'en général, les rayons font des angles plus ou moins grands, à pro- 
portion de la grandeur et de la distance des objets. Mais il ne lui serait pas possible de mesurer ces angles. $\mathrm{Si}$, comme il est vrai, les principes de l'optique sont insuffisants, pour expliquer la vision ; ils le sont à plus forte raison, pour nous apprendre à voir.

D'ailleurs cette science n'instruit point sur la manière, dont il faut mouvoir les yeux. Elle suppose seulement qu'ils sont capables de différents mouvements, et qu'ils doivent changer de forme, suivant les circonstances.

L'œil a donc besoin des secours du tact, pour se faire une habitude des mouvements propres à la vision ; pour s'accoutumer à rapporter ses sensations à l'extrémité des rayons, ou à-peu-près ; et pour juger par là des distances, des grandeurs, des situations et des figures. Il s’agit de découvrir ici quelles sont les expériences les plus propres à l'instruire.

\section{Elle sent les couleurs au bout de ses yeux.}

Soit hasard, soit douleur occasionnée par une lumière trop vive, la statue porte la main sur ses yeux ; à l'instant les couleurs disparaissent. Elle retire la main, les couleurs se reproduisent. Dès-lors elle cesse de les prendre pour ses manières d'être. Il lui semble que ce soit quelque chose d'impalpable, qu'elle sent au bout de ses yeux, comme elle sent au bout de ses doigts les objets qu'elle touche.

Mais, comme nous l'avons vu, chacune est une modification simple, qui ne donne par elle-même aucune idée distincte d'étendue : car une pareille idée serait celle d'une étendue figurée ou circonscrite ; et par conséquent elle serait une idée dont la statue, bornée au sens de la vue, était absolument privée. Une couleur ne représentera donc pas des dimensions à des yeux qui n'ont pas appris à la rapporter sur toutes les parties d'une surface : ils se sentiront seulement modifiés en eux-mêmes, et ils ne verront rien encore au-delà.

Mais, quoique les sensations de chaud et de froid ne portent avec elles aucune idée d'étendue, elles s'étendent cependant sur toutes les dimensions des corps, auxquels nous avons appris à les rapporter. C'est de la même manière que les couleurs s'étendront sur les objets : le toucher fera contracter aux yeux l'habitude de les juger sur une surface, comme il y juge lui-même le chaud ou le froid. 
Elle leur voit former une surface.

Parce que les couleurs sont enlevées à la statue, lorsqu'elle porte la main sur la surface extérieure de l'organe de la vue, c'est sur cette même surface, qu'elle croit d'abord les voir paraître ou disparaître : c’est-là qu'elle commence à leur donner de l'étendue.

Quand les corps s'éloignent ou s’approchent, elle ne juge donc point encore ni de leur distance, ni de leur mouvement. Elle aperçoit seulement des couleurs qui paraissent plus ou moins, ou qui disparaissent tout-à-fait.

Cette surface lui paraît immense.

Cette surface lumineuse est égale à la surface extérieure de l'œil : c'est tout ce que voit la statue ; et ses yeux n'aperçoivent rien au-delà, elle ne démêle donc point de bornes dans cette surface : elle la voit immense.

La statue n'a pas besoin d'apprendre à voir ;

mais elle a besoin d'apprendre à regarder.

Si nous offrons à sa vue une grande partie de l'horizon, la surface qu'elle verra sur ses yeux, pourra représenter une vaste campagne, variée par les couleurs et par les formes d'une multitude innombrable d'objets. La statue voit donc toutes ces choses : elle les voit, dis-je, mais elle n'en a point d'idée, et elle ne peut pas même en avoir d'aucune.

Cette proposition paraîtra sans doute un paradoxe à ceux qui décident que la vue seule, indépendamment du toucher, nous donne l'idée de l'étendue, puisque l'étendue est l'objet nécessaire de la vision; et que la différence des couleurs nous fera remarquer nécessairement les bornes ou limites qui séparent deux couleurs, et par conséquent nous donnera une idée de figure.

Il est certain que nous remarquons tout cela nous-mêmes, et je conviens que la statue voit tout ce que nous remarquons, et plus encore. Mais lorsqu'elle n'a pas appris du toucher à diriger ses yeux, estelle capable de remarquer ces choses comme nous ? Et en a-t-elle des idées, si elle ne les remarque pas? 
Il ne suffit pas de répéter, d'après Locke, que toutes nos connaissances viennent des sens : si je ne sais pas comment elles en viennent, je croirai qu'aussitôt que les objets font des impressions sur nous, nous avons toutes les idées que nos sensations peuvent renfermer, et je me tromperai. Voilà ce qui m’est arrivé, et ce qui arrive encore à tous ceux qui écrivent sur cette question. Il semble qu'on ne sache pas qu'il y a de la différence entre voir et regarder ; et cependant nous ne nous faisons pas des idées, aussitôt que nous voyons ; nous ne nous en faisons qu'autant que nous regardons et que nous regardons avec ordre, avec méthode. En un mot, il faut que nos yeux analysent : car ils ne saisiront pas l'ensemble de la figure la moins composée, s'ils n'en ont pas observé toutes les parties, séparément, l'une après l'autre, et dans l'ordre où elles sont entre elles. Or les yeux de la statue saventils analyser, lorsqu'ils ne voient encore les couleurs qu'en eux-mêmes ou tout au plus sur leur prunelle ? Voilà proprement à quoi se réduit la question. Je suis persuadé qu'un mathématicien, à qui on la proposerait, en se servant, comme je fais, du mot analyser, répondrait, sans balancer, que les yeux de la statue n'analysent pas ; car il se souvient combien il lui en a coûté à lui-même pour apprendre l'analyse. Mais si on la lui proposait avec le mot regarder, ce qui au fond n'y change rien, je crois qu'il répondrait également sans balancer, ses yeux regardent, puisqu'ils voient.

Il fera certainement cette réponse, s'il pense que les yeux seuls, indépendamment du toucher, nous donnent des idées de figure, aussitôt qu'ils voient des couleurs. Mais comment des yeux, dont la vue ne s’étend pas au-delà de leur prunelle, sauraient-ils regarder ? Car enfin, pour regarder, il faut qu'ils sachent se diriger sur un seul des objets qu'ils voient ; et pour se faire une idée de la figure de cet objet, quelque peu composée qu'elle soit, il faut qu'ils sachent se diriger sur chacune de ses parties, successivement et dans l'ordre où elles sont entre elles. Mais comment se dirigeront-ils en suivant un ordre qu'ils ne connaissent pas ? Comment même se dirigeront-ils sur quelque chose ? Cette action de leur part ne suppose-t-elle pas un espace, dans lequel ils recevraient les objets à différentes distances de leur prunelle, et à différentes distances entre eux, espace qu'ils ne connaissent pas encore ? Je ne dirai donc pas, comme tout le monde, et comme j'ai dit jusqu'à présent moi-même, et fort peu exactement, que nos yeux ont besoin d'apprendre à voir ; car ils voient nécessairement tout ce qui 
fait impression sur nous, mais parce qu'il ne suffit pas de voir pour se faire des idées, je dirai qu'ils ont besoin d'apprendre à regarder.

C'est de la différence qui est entre ces deux mots, que dépendait l'état de la question. Or pourquoi cette différence qui n'échappe pas aux plus petits grammairiens, échappe-t-elle aux philosophes ? Voilà donc comment nous raisonnons. Nous établissons mal l'état d'une question, nous ne savons pas l'établir, et cependant nous prétendons la résoudre ${ }^{24}$. Je viens de me prendre moi-même sur le fait, et j'avoue que je m’y suis pris souvent ; mais j’y prends plus souvent les autres.

Enfin quoiqu'il ait pu nous en coûter, voilà la question réduite à une question bien simple, et il est prouvé que les yeux de la statue ont besoin d'apprendre à regarder. Voyons comment le toucher les instruira.

\section{La statue juge cette surface loin d'elle.}

Par curiosité, ou par inquiétude, la statue continue de porter la main devant ses yeux : elle l'éloigne, elle l'approche ; et la surface qu'elle voit, en est plus lumineuse ou plus obscure. Aussitôt elle juge que le mouvement de sa main est la cause de ces changements; et comme elle sait qu'elle la meut à une certaine distance, elle soupçonne que cette surface n'est pas aussi près d'elle qu'elle l'a crue.

\section{Elle voit les couleurs sur les corps.}

Alors elle touche par hasard un corps qu'elle a devant les yeux, et le couvrant avec la main, et elle substitue une couleur à une autre. Elle laisse tomber les bras, la première couleur reparaît. Il lui semble donc que sa main fait, à une certaine distance, succéder ces deux couleurs.

Une autre fois elle la promène sur une surface, et voyant une couleur qui se meut sur une autre couleur, dont les parties paraissent et disparaissent tour-à-tour ; elle juge sur ce corps la couleur immobile, et sur sa main la couleur qui se meut. Ce jugement lui devient familier; et elle voit les couleurs s'éloigner de ses yeux, et se porter sur sa main et sur les objets qu'elle touche.

24 J'ai démontré dans ma logique que nous devons toutes nos idées à l'analyse ; et que toute question bien établie se résout en quelque sorte d’elle-même. 
Expériences qui achèvent de lui faire contracter cette habitude.

Étonnée de cette découverte, elle cherche autour d'elle, si elle ne touchera pas tout ce qu'elle voit. Sa main rencontre un corps d'une nouvelle couleur, son œil aperçoit une autre surface, et les mêmes expériences lui font porter les mêmes jugements.

Curieuse de découvrir s'il en est de même de toutes les sensations de cette espèce, elle porte la main sur tout ce qui l'environne ; et touchant un corps peint de plusieurs couleurs, son œil contracte l'habitude de les démêler sur une surface qu'il juge éloignée.

C'est sans doute par une succession de sentiments bien agréables pour elle, qu'elle conduit ses yeux dans ce chaos de lumières et de couleurs. Engagée par le plaisir, elle ne se lasse point de recommencer les mêmes expériences, et d'en faire de nouvelles. Elle accoutume peu-à-peu ses yeux à se fixer sur les objets qu'elle touche; ils se font une habitude de certains mouvements ; et bientôt ils percent comme à travers un nuage, pour voir dans l'éloignement les objets que la main saisit, et sur lesquels elle semble répandre la lumière et les couleurs.

Elle voit les objets à la distance où elle les

touche.

En conduisant tour-à-tour sa main de ses yeux sur les corps, et des corps sur ses yeux, elle mesure les distances. Elle approche ensuite ces mêmes corps, et les éloignent alternativement. Elle étudie les différentes impressions que son œil reçoit à chaque fois ; et s'étant accoutumée à lier ces impressions avec les distances connues par le tact, elle voit les objets tantôt plus près, tantôt plus loin, parce qu'elle les voit où elle les touche.

Elle apprend à voir un globe.

La première fois qu'elle porte la vue sur un globe, l'impression qu'elle en reçoit, ne représente qu'un cercle plat, mêlé d'ombre et de lumière. Elle ne voit donc pas encore un globe : car son œil n'a point encore appris à juger du relief sur une surface où l'ombre et la lumière sont distribuées dans une certaine proportion. Mais elle touche, et parce qu'elle apprend à porter avec la vue les mêmes jugements qu'elle 
porte avec le tact, ce corps prend sous ses yeux le relief qu'il a sous ses mains.

Elle réitère cette expérience, et elle répète le même jugement. Par là, elle lie les idées de rondeur et de convexité à l'impression que fait sur elle un certain mélange d'ombre et de lumière. Elle essaye ensuite de juger d'un globe, qu'elle n'a pas encore touché. Dans les commencements, elle s'y trouve sans doute quelquefois embarrassée : mais le tact lève l'incertitude ; et par l'habitude qu'elle se fait de juger qu'elle voit un globe, elle forme ce jugement avec tant de promptitude et d'assurance, et lie si fort l'idée de cette figure à une surface, où l'ombre et la lumière sont dans une certaine proportion, qu'enfin elle ne voit plus à chaque fois, que ce qu'elle s'est dit si souvent qu'elle doit voir.

Elle le distingue d'un cube.

Elle apprend également à voir un cube, lorsque ses yeux faisant une étude des impressions qu'ils reçoivent au moment que la main sent les angles et les faces de cette figure, elle contractera l'habitude de remarquer dans les différents degrés de lumière les mêmes angles et les mêmes faces ; et ce n'est qu'alors qu'elle discernera un globe d'un cube.

\section{Comment ses yeux sont en cela guidés par le} toucher.

L'œil ne parvient donc à voir distinctement une figure, que parce que la main lui apprend à en saisir l'ensemble. Il faut que, le dirigeant sur les différentes parties d'un corps, elle lui fasse donner son attention d'abord à une, puis à deux, peu-à-peu à un plus grand nombre ; et en même temps aux différentes impressions de la lumière. S'il n'étudiait pas séparément chaque partie, il ne verrait jamais la figure entière ; et s'il n'étudiait pas avec quelle variété la lumière agit sur lui, il ne verrait que des surfaces plates. Ainsi la statue ne parvient à voir tant de choses à-la-fois, que parce que les ayant remarquées séparément, elle se rappelle en un instant tous les jugements qu'elle a portés l'un après l'autre. 
Secours qu'ils tirent de la mémoire.

Notre expérience peut nous convaincre combien la mémoire est nécessaire pour parvenir à saisir l'ensemble d'un objet fort composé. Au premier coup-d'œil qu'on jette sur un tableau, on le voit fort imparfaitement : mais on porte la vue d'une figure à l'autre, et même on n'en regarde pas une toute entière. Plus on la fixe, plus l'attention se borne à une de ses parties : on n'aperçoit, par exemple, que la bouche.

Par là, nous contractons l'habitude de parcourir rapidement tous les détails du tableau ; et nous le voyons tout entier, parce que la mémoire nous présente à-la-fois tous les jugements, que nous avons portés successivement.

Mais cela est encore très borné à notre égard. Si j'entre, par exemple dans un grand cercle, il ne me donne d'abord qu'une idée vague de multitude. Je ne sais que je suis au milieu de dix ou douze personnes, qu'après les avoir comptées ; c'est-à-dire, qu'après les avoir parcourues une à une avec une lenteur, qui me fait remarquer la suite de mes jugements. Si elles n’avoient été que trois, je ne les aurais pas moins parcourues ; mais c'eût été avec une rapidité, qui ne m’eût pas permis de m'en apercevoir.

Si nos yeux n'embrassent une multitude d'objets qu'avec le secours de la mémoire, ceux de notre statue auront besoin du même secours, pour saisir l'ensemble de la figure la plus simple. Car n'étant pas exercés, cette figure est encore trop composée pour eux. La statue n'aura donc l'idée d’un triangle, qu'après l'avoir analysé.

Ils jugent des situations.

C'est la main, qui fixant successivement la vue sur les différentes parties d'une figure, les grave toutes dans la mémoire : c'est elle qui conduit, pour ainsi dire, le pinceau ; lorsque les yeux commencent à répandre au-dehors la lumière et les couleurs, qu'ils ont d'abord senties en eux-mêmes. Ils les aperçoivent où le toucher leur apprend qu'elles doivent être : ils voient en haut ce qu'il leur fait juger en haut, en bas ce qu'il leur fait juger en bas : en un mot, ils voient les objets dans la même situation, que le tact les représente. 
Le renversement de l'image n'y met aucun obstacle ; parce que tant qu'ils n’ont pas été instruits, il n’y a pas proprement pour eux ni haut ni bas. Le toucher, qui peut seul découvrir ces sortes de rapports, peut seul aussi leur apprendre à en juger.

D’ailleurs ne voyant au-dehors, que parce qu'ils rapportent les couleurs sur les objets que la main touche ; il faut nécessairement qu'ils s'accordent à porter sur les situations les mêmes jugements que le toucher.

Ils ne voient point double.

Chacun fixe l'objet que la main saisit, chacun rapporte les couleurs à la même distance, au même lieu; et comme le renversement de l'image ne leur empêche pas de voir un objet dans sa vraie situation, la même image, quoique double, ne leur empêche pas de le voir simple. La main les force à juger d'après ce qu'elle sent en elle-même. En les obligeant de rapporter au-dehors les sensations qu'ils éprouvent en eux, elle les leur fait rapporter à chacun sur l'unique objet qu'elle touche, et au seul endroit même où elle le touche. Il n'est donc pas naturel qu'ils le voient double.

Ils jugent des grandeurs.

Par la même raison, elle leur apprend au même instant à juger des grandeurs. Dès qu'elle leur fait voir les couleurs sur ce qu'elle touche, elle leur apprend à les étendre chacune sur toutes les parties qui les leur envoient ; elle dessine devant eux une surface, dont elle marque les bornes.

Ainsi, soit qu'elle éloigne ou qu'elle approche un objet, il leur paraît de la même grandeur, quoiqu'alors l'image augmente ou diminue ; comme il leur paraît simple et dans sa situation, quoique l'image soit double et renversée.

\section{Et du mouvement.}

Enfin, elle leur fait voir le mouvement des corps ; parce qu'elle les accoutume à suivre les objets, qu'elle fait passer d'un point de l'espace à l'autre. 
Ils ne voient pas encore hors de la portée de la

main.

Jusqu'ici la statue n'a étudié à la vue que les objets qui sont à la portée de sa main : car c'est par là qu'elle doit nécessairement commencer. Elle n'a donc point encore appris à voir au-delà, et elle se voit comme renfermée dans un court espace. A la vérité, le transport de son corps lui a appris que l'espace doit être beaucoup plus grand; mais elle n'imagine pas comment il pourra lui paraître aux yeux. En vain, se dirait-elle, il y a de l'étendue au-delà de celle que je vois : un pareil jugement ne peut la lui rendre visible. Ainsi qu'elle ne voit jusqu'à la portée de la main, que parce qu'ayant en même temps vu et touché à plusieurs reprises les objets qui sont dans cet espace ; elle a si fort lié les jugements du tact avec les sensations de lumière, que voir et juger se font tout-à-la-fois, et se confondent : elle ne verra plus loin, que lorsque de nouvelles expériences lui feront confondre avec ces mêmes sensations, les jugements qu'elle portera sur d'autres distances.

Elle aperçoit donc un espace, qui s'étend environ à deux pieds autour d'elle. Son œil instruit par le tact en mesure les parties, détermine la figure et la grandeur des objets qui y sont renfermés, les place à différentes distances, juge de leur situation, de leur mouvement et de leur repos.

Comment les objets qui sont au-delà, se montrent à eux.

Quant à ceux qui sont plus éloignés, elle les voit tous à l'extrémité de cette enceinte qui borne sa vue. Elle les aperçoit comme sur une surface lumineuse, concave et immobile. Ils lui paraissent figurés, parce que les expériences qu'elle a faites sur ceux qui sont à la portée de la main, suffisent à cet effet. S'ils se meuvent horizontalement, elle les voit passer d'une partie de la surface à l'autre : s'ils s'approchent ou s'ils s'éloignent d'elle, elle les voit seulement augmenter et diminuer d'une manière fort sensible. Mais elle ne juge point de leur vraie grandeur : car elle n'a appris à connaître à la vue les objets renfermés dans le court espace seul visible pour elle, que parce que le tact lui a fait lier différentes idées de grandeurs aux différentes impressions qui se font sur ses yeux. Or, ces impressions varient à proportion des dis- 
tances, puisque les images diminuent ou augmentent dans la même proportion. N'ayant donc fait aucune expérience pour lier ces impressions avec les grandeurs qui sont à quelques pas d'elle, elle ne peut juger des objets éloignés, que d'après les habitudes qu'elle a contractés. L'impression causée par de petites images, doit, par conséquent, les lui faire paraître petits, et l'impression causée par de grandes images, doit les lui faire paraître grands ; car c'est ainsi qu'elle juge de ceux que le tact a mis à la portée de ses yeux. Les liaisons qu'elle a formées pour juger à la vue des grandeurs qui sont à un pied ou à deux, ne suffisent donc pas pour juger de celles qui sont au-delà. Elles ne peuvent, à ce sujet, que la jeter dans l'erreur.

Cette surface qui termine sa vue, est précisément le même phénomène, que la voûte du ciel, à laquelle tous les astres semblent attachés, et qui paraît porter de tous côtés sur les extrémités des terres où la vue peut s'étendre. Elle la voit immobile, tant qu'elle l'est elle-même : elle la voit qui fuit devant elle, ou qui la suit, lorsqu'elle change de place. C'est ainsi que le ciel à l'horizon nous paraît se mouvoir.

\section{Ils apprennent à voir hors de la portée de la \\ main.}

Cependant, elle étend les bras pour saisir ce qu'elle voit. Surprise de ne rien toucher, elle avance. Enfin, elle rencontre un corps : aussitôt les jugements de la vue s'accordent avec ceux du tact. Un moment après, elle recule : d'abord l'objet ne lui paraît pas en être plus loin d'elle. Mais ayant essayé d'y porter la main, et n'ayant pu l'atteindre, elle va encore à lui ; et s'en étant éloignée et rapprochée à plusieurs reprises, elle s'accoutume peu-à-peu à le voir hors de la portée de la main.

Le mouvement qu'elle a fait pour s'en éloigner, lui donne à-peuprès une idée de l'espace qu'elle laisse entre elle et lui : elle sait quelle en était la grandeur, quand elle le touchait; et si le tact lui a appris à le voir à deux pieds d'une certaine grandeur, le souvenir qui lui reste de cette grandeur, lui apprend à la lui conserver à une plus grande distance.

Alors elle peut juger à la vue s'il s'éloigne ou s'il s'approche, ou s'il se meut dans quelqu'autre direction ; car elle en voit les mouvements dans les changements qui arrivent aux impressions qui se font 
sur ses yeux. Il est vrai que ces changements sont les mêmes, soit qu'elle aille à lui, ou qu'il vienne à elle, soit qu'elle passe devant lui dans une certaine direction, ou qu'il passe devant elle dans une direction contraire ; mais le sentiment qu'elle a de son propre mouvement ou de son propre repos, ne lui permet pas de s'y tromper.

Elle s’accoutume donc à lier différentes idées de distance, de grandeur et de mouvement aux différentes impressions de lumière. Elle ne sait pas, à la vérité, que les images qui se tracent au fond de l'œil, diminuent à proportion des distances. Elle ne sait pas même s’il y a de pareilles images. Mais elle éprouve des sensations différentes, et les jugements dont elle se fait une habitude suivant les circonstances, venant à se confondre avec ces sensations, ce n'est plus dans ses yeux qu'elle sent la lumière et les couleurs ; elle les sent à l'autre extrémité des rayons, comme elle sent la solidité, la fluidité, etc. Au bout du bâton avec lequel elle touche les corps.

Ainsi plus ses yeux règlent leurs jugements d'après les leçons du toucher, plus l'espace leur paraît prendre de profondeur. Elle aperçoit la lumière et les couleurs, qui, répandues sur les objets, en dessinent la grandeur, la figure, en tracent le mouvement dans l'espace ; en un mot, elle les voit où elle juge qu'elles doivent être.

Pourquoi les objets qui s'éloignent, leur paraissent diminuer insensiblement.

Cependant, quelque souvenir qu'elle ait de la grandeur d'un objet, elle ne peut l'empêcher de diminuer à ses yeux, à mesure qu'il s'éloigne d'elle. Voici la raison de ce phénomène.

Un objet n'est visible, qu'autant que l'angle, qui détermine l'étendue de son image sur la rétine, est d'une certaine grandeur. Je suppose qu'il doive être au moins d'une minute : mais c'est uniquement pour fixer nos idées ; car la chose doit varier suivant les yeux.

Dans cette supposition, on conçoit aisément, qu'un objet vu distinctement à une certaine distance, ne peut s'éloigner, qu'à chaque instant les angles, qui faisaient voir les moindres parties, ne deviennent plus petits, et que plusieurs ne se trouvent au-dessous d'une minute. Il faut même que dans quelques-uns les côtés se rapprochent au point de se confondre en une seule ligne. Ainsi de plusieurs angles il 
s'en formera un, dont les côtés se confondront encore, si l'objet continue à s'éloigner. Il y aura donc des parties qui cesseront de se retracer sur la rétine. Elles se ramasseront, se pénétreront, se confondront avec celles qui se peindront encore ; et les extrémités de l'objet se rapprocheront. L'image, par exemple, de la tête d'un homme se fera sans distinction de traits.

Or, le toucher n’apprend à l'œil à voir les objets dans leur véritable grandeur, que parce qu'il lui apprend à en démêler les parties, et à les apercevoir les unes hors des autres. C'est ce qu'il ne peut faire, qu'autant qu'elles sont tracées distinctement sur la rétine. Car les yeux ne sauraient parvenir à remarquer dans leurs sensations ce qui n'y serait pas. Ils doivent donc juger un objet plus ramassé et plus petit, quand il est dans un éloignement, ou quantité de traits de son image se confondent. Par conséquent, à quelque distance que soit un objet, il continue de paraître de la même grandeur, tant que la diminution des angles n'altère pas sensiblement l'image qui se peint sur la rétine ; et c'est parce que cette altération se fait par des degrés insensibles, qu'un objet qui s’éloigne, paraît diminuer insensiblement.

\section{Comment ils apprennent à se passer du} secours du tact.

Non-seulement les yeux de la statue démêlent les objets qu'elle ne touche plus, ils démêlent encore ceux qu'elle n’a pas touchés ; pourvu qu'ils en reçoivent des sensations semblables, ou à-peu-près. Car le tact ayant une fois lié différents jugements à différentes impressions de lumière, ces impressions ne peuvent plus se reproduire, que les jugements ne se répètent, et ne se confondent avec elles. C'est ainsi qu'elle s'accoutume peu-à-peu à voir sans le secours du toucher.

\section{Pourquoi ils se tromperont.}

Cependant, les expériences qui lui ont appris à voir la distance, la grandeur la figure d'un corps, ne suffiront pas toujours pour lui apprendre à voir la distance, la grandeur et la figure de tout autre. Il faut qu'elle fasse autant d'observations qu'il y a d'objets qui réfléchissent différemment la lumière ; il faut même que sur chaque objet, elle multiplie ses observations suivant les différents degrés de distance ; et en- 
core, malgré toutes ces précautions, se trompera-t-elle souvent sur les grandeurs, sur les distances et sur les figures.

Ce n'est, par conséquent, qu'après bien des études, qu'elle commencera à s'assurer mieux des jugements de sa vue : mais il lui sera impossible d'éviter absolument toute méprise. Souvent elle sera trompée par les expériences mêmes, auxquelles elle croit devoir se fier davantage. Accoutumée, par exemple, à lier l’idée de proximité à la vivacité de la lumière, et l'idée de l'éloignement à son obscurité ; quelquefois des corps lumineux lui paraîtront plus proches qu'ils ne sont, et au contraire, des corps peu éclairés lui paraîtront plus éloignés.

Ils seront en contradiction avec le toucher.

Il pourrait même arriver à ses yeux d'être en contradiction, au point de ne pouvoir plus s'accorder à porter avec lui les mêmes jugements. Ils verront, par exemple, de la convexité sur un relief peint, où la main n’apercevra qu'une surface plate. Sans doute étonnée de ce nouveau phénomène, elle ne sait lequel croire de ces deux sens : en vain le tact relève l'erreur de la vue; les yeux accoutumés à juger par eux-mêmes, ne consultent plus leur maître. Ayant appris de lui à voir d'une manière, ils ne peuvent plus apprendre à voir différemment.

En effet, ils ont contracté une habitude qui ne peut leur être enlevée ; parce que les jugements qui leur font voir de la convexité dans une certaine impression d'ombre et de lumière, sont devenus naturels. Car ayant été faits à bien des reprises, ils se répètent rapidement, et se confondent avec la sensation, toutes les fois que la même impression d'ombre et de lumière a lieu.

Si l'on disposait les choses de manière que parmi les objets que notre statue aurait occasion de toucher, il y eût autant de reliefs peints sur des surfaces plates, que de corps véritablement convexes ; elle serait fort embarrassée pour distinguer à la vue ceux qui ont de la convexité, de ceux qui n'en ont pas. Elle y serait trompée si souvent, qu'elle n'oserait s'en rapporter à ses yeux ; elle n'en croirait plus que le toucher.

Une glace mettrait encore ces deux sens en contradiction. La statue ne douterait pas qu'il n'y eût au-delà un grand espace. Elle serait fort étonnée d'être arrêtée par un corps solide, et elle le serait encore au- 
tant, lorsqu'elle commencerait à reconnaître les objets qu'il lui répète. Elle n'imagine pas comment ils se doublent à la vue ; et elle ne sait pas s'ils ne pourraient pas aussi se doubler au tact.

Et même avec eux.

Non seulement la vue sera en contradiction avec le toucher, elle le sera encore avec elle-même. La statue juge, par exemple, qu'une tour est ronde et fort petite, quand elle en est à une certaine distance. Elle approche, et elle en voit sortir des angles, elle la voit grandir à ses yeux. Se trompe-t-elle, ou s'est-elle trompée ? C'est ce qu'elle ne saura, que lorsqu'elle sera à portée de toucher la tour. Ainsi le tact, qui seul a instruit les yeux, peut aussi lui seul faire discerner les occasions où l'on peut compter sur leur témoignage.

Ils jugent de la distance par la grandeur.

Mais si la statue est privée de ce secours, elle s’aidera de toutes les connaissances qu'elle a acquises. Tantôt elle jugera de la distance par la grandeur. Un objet lui paraît-il aussi grand à la vue qu'au toucher, elle le voit près ; lui paraît-il plus petit, elle le voit loin. Car elle a remarqué que les apparences des grandeurs varient suivant les distances.

Par la netteté des images.

D’autres fois, elle détermine les distances par le degré de netteté des figures qui s'offrent à ses yeux. Ayant souvent observé qu'elle voit plus confusément les objets qui sont éloignés, et plus distinctement ceux qui sont proches; elle lie l'idée d'éloignement à la vue confuse d'une figure, et l'idée de proximité à la vue distincte. Elle prend donc l'habitude de voir un objet fort loin, quand elle le voit peu distinctement ; et de le voir près, quand elle en distingue mieux les parties.

Ils jugent des grandeurs par la distance.

Alors jugeant de la grandeur par la distance, comme elle juge dans d'autres occasions de la distance par la grandeur, elle voit plus grand ce qu'elle croit plus loin. Deux arbres, par exemple, qui lui enverront des images de même étendue, ne lui paraîtront point égaux, ni à la 
même distance, si l'un se peint plus confusément que l'autre : elle verra plus grand et plus loin celui où elle discernera moins de choses. Une mouche encore lui paraîtra un oiseau dans l'éloignement, si passant rapidement devant ses yeux, elle ne laisse apercevoir qu'une image confuse, semblable à celle d'un oiseau éloigné.

Ces principes sont connus de tout le monde, et la peinture les confirme. Un cheval qui occupe sur la toile le même espace qu'un mouton, paraîtra plus grand et dans l'enfoncement, pourvu qu'il soit peint d'une manière plus confuse.

C'est ainsi que les idées de distance, de grandeur et de figure, d'abord acquises par le toucher, se prêtent ensuite des secours, pour rendre les jugements de la vue plus sûre.

Ils jugent des distances et des grandeurs par les objets intermédiaires.

Notre statue voyant l'espace prendre de la profondeur à ses yeux, a encore un moyen pour connaître avec plus de précision les distances, et par conséquent les grandeurs. C'est de porter la vue sur les objets, qui sont entre elle et celui qu'elle fixe. Elle le voit plus loin et plus grand, si elle en est séparée par des champs, des bois, des rivières. Car l'étendue des champs, des bois et des rivières lui étant connue, c'est une mesure qui détermine combien elle en est éloignée. Mais si quelque élévation lui cache les objets intermédiaires, elle ne jugera de sa distance, qu'autant que quelque circonstance lui en rappellera la grandeur. Un cheval immobile peut, par exemple, lui paraître assez petit et assez près. Il se meut : à ses mouvements elle le reconnaît : aussitôt elle le juge de la grandeur ordinaire, et elle l'aperçoit dans l'éloignement.

Elle le croit d'abord assez petit et assez près, parce qu'aucun objet intermédiaire ne lui en fait voir la distance, et qu'aucune circonstance ne lui apprend ce que ce peut être. Mais dès que le mouvement le lui fait reconnaître, elle le voit à-peu-près de la grandeur qu'elle sait appartenir à cet animal ; et elle le voit loin d'elle, parce qu'elle juge que l'éloignement est la seule cause qui ait pu le rendre si confus à ses yeux. 
Cas où ils ne jugent plus des grandeurs ni des distances.

Avec ces secours, elle discerne donc assez bien à l'œil les distances : mais elle n’y réussit plus, aussitôt qu'ils viennent à lui manquer ; et sa vue est bornée là, où elle cesse de voir des objets intermédiaires, et où elle n'aperçoit que des corps, dont le tact ne lui a pas appris la grandeur. Les cieux lui paraissent former une voûte, qui ne s'élève pas au-dessus des montagnes, et qui ne s'étend pas au-delà des terres que son œil embrasse. Faites-lui voir d'autres objets au-dessus de ces montagnes et au-delà de ces terres ; cette voûte aura plus de hauteur et plus d'étendue. Mais elle en aurait eu moins, si on avait supposé les montagnes moins élevées, et les terres resserrées dans des bornes plus étroites. Le faîte d'un arbre lui aurait paru toucher le ciel.

Ce phénomène est donc, comme nous l'avons dit, le même que celui qui bornait sa vue à deux pieds d'elle : et puisque n'ayant aucun moyen pour juger de l'éloignement des astres, ils lui paraissent tous à la même distance ; c'est une preuve que dans la supposition, que nous avons faite plus haut, tous les objets ont dû lui paraître à la portée de sa main.

\section{Effets qui résultent des grandeurs comparées.}

Cependant, familiarisée avec les grandeurs, elle les compare ; et cette comparaison influe sur les jugements qu'elle en porte. Dans les commencements elle ne juge pas un objet absolument grand, ni absolument petit ; mais elle en juge par rapport à des grandeurs, qui lui étant plus familières, sont à son égard la mesure de toutes les autres. Elle voit grand, par exemple, tout ce qui est au-dessus de sa hauteur, et petit tout ce qui est au-dessous. Ces comparaisons se font ensuite si rapidement, qu'elle ne les remarque plus ; et dès-lors la grandeur et la petitesse deviennent pour elle des idées absolues. Une pyramide de vingt pieds, qu'elle aura trouvée absolument grande à côté d'une de dix, elle la jugera absolument petite à côté d'une de quarante ; et elle ne soupçonnera pas que ce soit la même.

Au reste, il n’est pas nécessaire pour ces expériences, que les objets soient de même espèce : il suffit que l'œil ait occasion de comparer grandeur à grandeur. C'est pourquoi dans une plaine fort étendue, 
les mêmes objets lui paraîtront plus petits, que dans un pays coupé par des coteaux.

Cette manière de comparer les grandeurs est encore une cause qui contribue à les diminuer aux yeux, suivant qu'elles sont plus éloignées, et surtout plus élevées. Car l'œil ne peut suivre un objet qui fuit devant lui, ou qui s'élève dans l'air, qu'il ne le compare avec un plus grand espace, à proportion qu'il le voit à une plus grande distance.

L'entier usage de la vue nuit à la sagacité des autres sens.

Tels sont les moyens par où la statue apprendra à juger à la vue de l'espace, des distances, des situations, des figures, des grandeurs et du mouvement. Plus elle se sert de ses yeux, plus l'usage lui en devient commode. Ils enrichissent la mémoire des plus belles idées, suppléent à l'imperfection des autres sens, jugent des objets qui leur sont inaccessibles, et se portent dans un espace, auquel l'imagination peut seule ajouter. Aussi leurs idées se lient si fort à toutes les autres, qu'il n'est presque plus possible à la statue de penser aux objets odoriférants, sonores, ou palpables, sans les revêtir aussitôt de lumière et de couleur. Par l'habitude qu'ils contractent de saisir tout un ensemble, d'en embrasser même plusieurs, et de juger de leurs rapports ; ils acquièrent un discernement si supérieur, que la statue les consulte par préférence. Elle s'applique donc moins à reconnaître au son les situations et les distances, à discerner les corps par les nuances des odeurs qu'ils exhalent, ou par les différences que la main peut découvrir sur leur surface. L'ouïe, l'odorat et le toucher en sont, par conséquent, moins exercés. Peu-à-peu devenus plus paresseux, ils cessent d'observer dans les corps toutes les différences qu'ils y démêlaient auparavant ; et ils perdent de leur finesse, à proportion que la vue acquiert plus de sagacité. 


\section{Chapitre IV}

Pourquoi on est porté à attribuer à la vue des idées qu'on ne doit qu'au toucher. Par quelle suite de réflexions on est parvenu à détruire ce préjugé

Pourquoi on a de la peine à se persuader que l'œil a besoin d'apprentissage.

Il nous est devenu si naturel de juger à l'œil des grandeurs, des figures, des distances et des situations, qu'on aura peut-être encore bien de la peine à se persuader que ce ne soit là qu'une habitude due à l'expérience. Toutes ces idées paraissent si intimement liées avec les sensations de couleur, qu'on n'imagine pas qu'elles en aient jamais été séparées. Voilà, je pense, l'unique cause qui peut retenir dans le préjugé. Mais pour le détruire tout-à-fait, il suffit de faire des suppositions semblables à celles que nous avons déjà faites.

Suppositions qui achèvent de détruire ce préjugé.

Notre statue croirait infailliblement que les odeurs et les sons lui viennent par les yeux, si, lui donnant tout-à-la-fois la vue, l'ouïe et l'odorat, nous supposions que ces trois sens fussent toujours exercés ensemble ; en sorte qu'à chaque couleur qu'elle verrait, elle sentît une certaine odeur, et entendît un certain son ; et qu'elle cessât de sentir et d'entendre, lorsqu'elle ne verrait rien.

C'est donc parce que les odeurs et les sons se transmettent, sans se mêler avec les couleurs, qu'elle démêle si bien ce qui appartient à l'ouïe et à l'odorat. Mais comme le sens de la vue et celui du toucher agissent en même temps, l'un pour nous donner les idées de lumière et de couleur, l'autre pour nous donner celles de grandeur, de figure, de distance et de situation ; nous distinguons difficilement ce qui appar- 
tient à chacun de ces sens, et nous attribuons à un seul ce que nous devrions partager entre eux.

Ainsi la vue s'enrichit aux dépens du toucher, parce que n’agissant qu'avec lui, ou qu'en conséquence des leçons qu'elle en a reçues, ses sensations se mêlent avec les idées, qu'elle lui doit. Le tact au contraire agit souvent seul, et ne nous permet pas d'imaginer que les sensations de lumière et de couleur lui appartiennent.

Mais si la statue ne voyait jamais que les corps qu'elle toucherait, et ne touchait jamais que ceux qu'elle verrait, il lui serait impossible de discerner les sensations de la vue de celles du toucher. Elle ne soupçonnerait seulement pas qu'elle eût des yeux. Ses mains lui paraîtraient voir et toucher tout ensemble.

Ce sont donc des jugements d'habitude, qui nous font attribuer à la vue des idées, que nous ne devons qu'au tact.

\section{Soupçons et réflexions qui ont amené cette} découverte.

Il me semble que lorsqu'une découverte est faite, il est curieux de connaître les premiers soupçons des philosophes, et surtout les réflexions de ceux qui ont été sur le point de saisir la vérité.

\section{De Malebranche.}

Malebranche est, je crois, le premier, qui ait dit qu'il se mêle des jugements dans nos sensations. Il remarque que bien des lecteurs seront choqués de ce sentiment. Mais ils le seront surtout, quand ils verront les explications que ce philosophe en donne. Car il n'évite un préjugé, que pour tomber dans une erreur. Ne pouvant comprendre comment nous formerions nous-mêmes ces jugements, il les attribue à Dieu : manière de raisonner fort commode, et presque toujours la ressource des philosophes.

« Je crois devoir avertir, dit-il ${ }^{25}$, que ce n'est point notre âme qui forme les jugements de la distance, de la grandeur, etc., des objets ; ... mais que c'est Dieu, en conséquence des lois de l'union de l'âme et du corps. C'est pour cela que j'ai appelé naturels ces sortes de juge-

25 Recher. de la Vér., L. 1. C. 9. 
ments, pour marquer qu'ils se font en nous, sans nous et malgré nous... Dieu seul peut nous instruire en un instant de la grandeur, de la figure, du mouvement et des couleurs des objets qui nous environnent. »

Il explique encore plus au long dans un éclaircissement sur l'optique ${ }^{26}$, comment il imagine que Dieu forme pour nous ces jugements.

De Locke.

Locke n'était pas capable de faire de pareils systèmes. Il reconnaît que nous ne voyons des figures convexes qu'en vertu d'un jugement que nous formons nous-mêmes, et dont nous nous sommes fait une habitude. Mais la raison qu'il en donne n'est pas satisfaisante.

«Comme nous nous sommes, dit-il ${ }^{27}$, accoutumés par l'usage à distinguer quelle sorte d'image les corps convexes produisent ordinairement en nous, et quels changements arrivent dans la réflexion de la lumière, selon la différence de la figure sensible des corps, nous mettons aussitôt à la place de ce qui nous paraît, la cause même de l'image que nous voyons, et cela en vertu d'un jugement que la coutume nous a rendu habituel ; de sorte que joignant à la vision un jugement que nous confondons avec elle, nous nous formons l'idée d'une figure convexe... »

Peut-on supposer que les hommes connaissent les images que les corps convexes produisent en eux, et les changements qui arrivent dans la réflexion de la lumière, selon la différence des figures sensibles des corps?

$\mathrm{N}^{\mathrm{os}} 26$ et 43.

27 Essai philos., L. 2. C. 9. § 8. 
De Molyneux.

Molyneux, en proposant un problème qui a donné occasion de développer tout ce qui concerne la vue, paraît n'avoir saisi qu'une partie de la vérité.

«Supposez, lui fait dire Locke ${ }^{28}$, un aveugle de naissance, qui soit présentement homme fait, auquel on ait appris à distinguer par l'attouchement un globe et un cube de même métal, et à-peu-près de même grosseur... On demande si, en les voyant, il pourra les discerner? »

Les conditions que les deux corps soient de même métal et de même grosseur, sont superflues; et la dernière paraît supposer que la vue peut, sans le secours du tact, donner différentes idées de grandeur. Cela étant, on ne voit pas pourquoi Locke et Molyneux nient qu'elle puisse toute seule discerner les figures.

D’ailleurs, ils auraient dû raisonner sur les distances, les situations et les grandeurs, comme sur les figures ; et conclure, qu'au moment où un aveugle-né ouvrirait les yeux à la lumière, il ne jugerait d'aucune de ces choses. Car elles se retrouvent toutes en petit dans la perception des différentes parties d'un globe et d'un cube. C'est se contredire, que de supposer qu'un œil, qui discernerait les situations, les grandeurs et les distances, ne saurait discerner les figures.

\section{De Barclay.}

Le docteur Barclay est le premier qui ait pensé que la vue par ellemême ne jugerait d'aucune de ces choses.

Une autre conséquence qui n'aurait pas dû échapper à Locke, c'est que des yeux sans expérience, ne verraient qu'en eux-mêmes la lumière et les couleurs ; et que le tact peut seul leur apprendre à voir audehors.

28 Ibid. 
Enfin, Locke aurait dû remarquer qu'il se mêle des jugements dans toutes nos sensations, par quelque organe qu'elles soient transmises à l’âme. Mais il dit précisément le contraire ${ }^{29}$.

Tout cela prouve qu'il faut bien du temps, bien des méprises et bien des demi-vues, avant d'arriver à la vérité. Souvent on est tout auprès, et on ne sait pas la saisir.

Table des matières 


\section{Chapitre V}

\section{D’un aveugle-né, à qui les cataractes ont été abaissées}

L’aveugle-né ne voulait pas se prêter à

l’opération.

Cheselden, fameux chirurgien de Londres, a eu plusieurs fois occasion d'observer des aveugles-nés, à qui il a abaissé les cataractes. Comme il a remarqué, que tous lui ont à-peu-près dit les mêmes choses ; il s'est borné à rendre compte de celui dont il a tiré le plus de détails ${ }^{30}$.

C'était un jeune homme de treize à quatorze ans. Il eut de la peine à se prêter à l'opération ; il n'imaginait pas ce qui pouvait lui manquer. En connaîtrai-je mieux, disait-il, mon jardin ? M'y promèneraije plus librement? D’ailleurs, n'ai-je pas sur les autres l'avantage d'aller la nuit avec plus d'assurance ? C'est ainsi que les compensations qu'il trouvait dans son état lui faisaient présumer qu'il était tout aussi bien partagé que nous. En effet, il ne pouvait regretter un bien qu'il ne connaissait pas.

Invité à se laisser abattre les cataractes, pour avoir le plaisir de diversifier ses promenades, il lui paraissait plus commode de rester dans les lieux qu'il connaissait parfaitement ; car il ne pouvait pas comprendre qu'il pût jamais lui être aussi facile de se conduire à l'œil dans ceux où il n'avait pas été. Il n'eût donc point consenti à l'opération, s’il n'eût souhaité de savoir lire et écrire. Ce seul motif le décida ; et l'on commença par abaisser la cataracte à l'un de ses yeux.

30 Transactions Philos., N. 402, an. 1728. 
État de ses yeux avant l'opération.

Il faut remarquer qu'il n'était point si aveugle, qu'il ne distinguât le jour d'avec la nuit. Il discernait même à une grande lumière, le blanc, le noir et le rouge. Mais ces sensations étaient si différentes de celles qu'il eut dans la suite, qu’il ne les put pas reconnaître.

Après l'opération, les objets lui paraissent au bout de l'oeil.

Quand il commença à voir, les objets lui parurent toucher la surface extérieure de son œil. La raison en est sensible.

Avant qu'on lui abaissât les cataractes, il avait souvent remarqué, qu'il cessait de voir la lumière, aussitôt qu'il portait la main sur ses yeux. Il contracta donc l'habitude de la juger au-dehors. Mais parce que c'était une lueur faible et confuse, il ne discernait pas assez les couleurs, pour découvrir les corps qui les lui envoyaient. Il ne les jugeait donc pas à une certaine distance ; il ne lui était donc pas possible d’y démêler de la profondeur : et, par conséquent, elles devaient lui paraître toucher immédiatement ses yeux. Or, l'opération ne put produire d'autre effet, que de rendre la lumière plus vive et plus distincte. Ce jeune homme devait donc continuer de la voir, où il l'avait jugée jusqu'alors, c'est-à-dire, contre son œil.

Par conséquent, il n’apercevait qu'une surface égale à la grandeur de cet organe.

Et fort grands.

Mais il prouva la vérité des observations que nous avons faites ${ }^{31}$ : car tout ce qu'il voyait, lui paraissait d'une grandeur étonnante. Son œil n’ayant point encore comparé grandeur à grandeur, il ne pouvait avoir à ce sujet des idées relatives. Il ne savait donc point encore démêler les limites des objets, et la surface, qui le touchait, devait, comme à la statue, lui paraître immense. Aussi nous assure-t-on qu'il fut quelque temps, avant de concevoir qu'il y eût quelque chose audelà de ce qu'il voyait.

31 Part. 3. C. 3. § 5. 
Il ne les discerne ni à la forme, ni à la grandeur.

Il apercevait tous les objets pêle-mêle et dans la plus grande confusion, et il ne les distinguait point, quelques différentes qu'en fussent la forme et la grandeur. C'est qu'il n'avait point encore appris à saisir à la vue plusieurs ensembles. Comment l'aurait-il appris ? Ses yeux, qui n’avaient jamais rien analysé, ne savaient pas regarder, ni par conséquent remarquer différents objets, et se faire de chacun des idées distinctes..

Mais à mesure qu'il s'accoutuma à donner de la profondeur à la lumière, et à créer, pour ainsi dire, un espace au-devant de ses yeux ; il plaça chaque objet à différentes distances, assigna à chacun le lieu qu'il devait occuper, et commença à juger à l'œil de leur forme et de leur grandeur relative.

Il n'imagine pas comment l'un peut être à la vue plus petit que l'autre.

Tant qu'il ne se fut point encore familiarisé avec ces idées, il ne les comparaît que difficilement, et il était bien éloigné d'imaginer comment les yeux pourraient être juges des rapports de grandeur. C'est pourquoi n'étant point encore sorti de sa chambre, il disait, que quoiqu'il la sût plus petite que la maison, il ne comprenait pas comment elle pourrait le lui paraître à la vue. En effet, son œil n'avait point fait jusque-là de comparaisons de cette espèce. C'est aussi par cette raison, qu'un objet d'un pouce, mis devant son œil, lui paraissait aussi grand que la maison.

\section{Il n’apprend à voir qu’à force d'étude.}

Des sensations aussi nouvelles, et dans lesquelles il faisait à chaque instant des découvertes, ne pouvaient manquer de lui donner la curiosité de tout voir, et de tout étudier à l'œil. Aussi lorsqu'on lui montrait des objets qu'il reconnaissait au toucher; il les observait avec soin, pour les reconnaître une autre fois à la vue. Il y apportait même d'autant plus d'attention, qu'il ne les avait d'abord reconnus ni à leur forme ni à leur grandeur : mais il avait tant de choses à retenir, qu'il oubliait la manière de voir quelques objets, à mesure qu'il ap- 
prenait à en voir d'autres. J'apprends, disait-il, mille choses en un jour, et j'en oublie tout autant.

\section{Objets qu'il voyait avec plus de plaisir.}

Dans cette situation, les objets qui réfléchissent le mieux la lumière, et dont l'ensemble se saisit plus facilement, devaient lui plaire plus que les autres. Tels sont les corps polis et réguliers. Aussi nous assuret-on qu'ils lui paraissaient les plus agréables : mais il ne put en rendre raison. Ils lui plaisaient même déjà davantage dans un temps, où il ne savait point encore bien dire quelle en était la forme ${ }^{32}$.

\section{Son étonnement à la vue d'un relief peint.}

Comme le relief des objets n'est pas aussi sensible dans la peinture, que dans la réalité, ce jeune homme fut quelque temps à ne regarder les tableaux, que comme des plans différemment colorés : ce ne fut qu'au bout de deux mois, qu'ils lui parurent représenter des corps solides ; et ce fut une découverte, qu'il parut faire tout-à-coup. Surpris de ce phénomène, il les regardait, il les touchait ; et il demandait quel est le sens qui me trompe ? Est-ce la vue ou le toucher ?

\section{A la vue d'un portrait en miniature.}

Mais un prodige pour lui, ce fut le portrait en miniature de son père. Cela lui paraissait aussi extraordinaire, que de mettre un muid dans une pinte : c'était son expression. Son étonnement avait pour cause l'habitude que son œil avait prise, de lier la forme à la grandeur d'un

32 Je crois devoir avertir que ce n'est pas là précisément ce que rapporte Cheselden. Car en même temps qu'il dit que ce jeune homme ne pouvait discerner les objets, quelque différentes qu'en fussent la forme et la grandeur, il assure qu'il trouvait beaucoup plus agréables ceux qui étaient réguliers. Pour moi, cela me paraît tout-à-fait contradictoire ; et Cheselden ne s'est pas expliqué avec assez de soin. Il était naturel que ce jeune homme ne distinguât ni forme ni grandeur, au premier moment qu'il vit la lumière ; mais il ne lui eût pas été possible de trouver plus de plaisir à voir des objets réguliers, si sa vue eût continué d’être aussi confuse. Il n’a donc pu les juger plus agréables, que lorsqu'il commençait à démêler des formes et des grandeurs. Il avait sans doute de la peine à expliquer à ses observateurs les différences qu'il remarquait alors : et c'est peut-être ce qui a fait juger qu'elles lui avaient échappé jusqu’à ce moment. 
objet. Il ne s'était pas encore accoutumé à juger que ces deux choses peuvent être séparées.

Prévention où il était.

Nous avons du penchant à nous prévenir, et nous présumons volontiers que tout est bien dans un objet, qui nous a plu par quelqu'endroit. Aussi ce jeune homme paraissait-il surpris que les personnes qu'il aimait le mieux, ne fussent pas les plus belles: et que les mets qu'il goûtait davantage, ne fussent pas les plus agréables à l'œil.

Il y avait pour lui plusieurs manières de voir.

Plus il exerçait sa vue, plus il se félicitait d'avoir consenti à se laisser abaisser la cataracte ; et il disait que chaque nouvel objet était pour lui un délice nouveau. Il parut surtout enchanté, lorsqu'on le conduisit à Epsom, où la vue est très belle et très étendue. Il appelait ce spectacle une nouvelle manière de voir. Il n'avait pas tort ; car il y a en effet autant de manières de voir, qu'il entre de jugements différents dans la vision : et combien n'y en doit-il pas entrer à la vue d'une campagne fort vaste et fort variée ! Il le sentait mieux que nous, parce qu'il les formait avec peu de facilité.

\section{Le noir lui était désagréable.}

On remarque que le noir lui était désagréable, et que même il se sentit saisi d'horreur, la première fois qu'il vit un nègre, c'est peutêtre parce que cette couleur lui rappelait son premier état.

Comment il vit, lorsque l'opération eut été

faite sur les deux yeux.

Enfin, plus d'un an après, on fit l'opération sur l'autre œil, et elle réussit également. Il vit de cet œil tout en grand, mais moins qu'il n’avait fait avec le premier. Je crois démêler la raison de cette différence. C’est que ce jeune homme prévenu qu'il devait voir de la même manière avec celui-ci, mêla aux sensations qu'il lui transmettait, les jugements dont il s'était fait une habitude avec celui, par où on avait commencé l'opération. Mais comme il n’y pouvait pas porter du premier coup la même précision, il vit de cet œil les objets encore trop 
grands. La même prévention put aussi les lui faire voir moins confusément, qu'il n'avait fait avec le premier. Mais on n’en dit rien.

Lorsqu'il commença à regarder un objet des deux yeux, il crut le voir une fois plus grand. C'est qu'il était plus naturel que l'œil, qui voyait en petit, ajoutât aux grandeurs qu'il apercevait, qu'il n'était naturel que celui qui voyait en grand, en retranchât.

Mais ses yeux ne virent point double ; parce que le toucher, en apprenant à celui qui venait de s’ouvrir à la lumière, à démêler les objets, les lui fit voir où il les faisait voir à l'autre.

\section{Difficulté qu'il avait à diriger ses yeux.}

$\mathrm{Au}$ reste, Cheselden remarque que ce qui embarrassait beaucoup les aveugles-nés, à qui il a abaissé les cataractes ; c'était de diriger les yeux sur les objets qu'ils voulaient regarder. Cela devait être : jusqu'alors n'ayant pas eu besoin de les mouvoir, ils n'avaient pu se faire une habitude de les conduire, et cela confirme ce que j'ai démontré.

Il n'est pas possible qu'il n’y ait des choses à désirer dans des observations qu'on fait pour la première fois sur des phénomènes, où il entre mille détails difficiles à saisir. Mais elles servent au moins à donner des vues pour observer une autre fois avec plus de succès. Je hasarderai les miennes dans le chapitre suivant. 


\section{Chapitre VI}

\section{Comment on pourrait observer un aveugle-né, à qui on abaisserait les cataractes}

Précaution à prendre.

Une précaution à prendre avant l'opération des cataractes, ce serait de faire réfléchir l'aveugle-né sur les idées qu'il a reçues par le toucher ; en sorte qu'étant en état d'en rendre compte, il pût assurer, si la vue les lui transmet, et dire de lui-même ce qu'il voit, sans qu'on fût presque obligé de lui faire des questions.

\section{Observations à faire.}

Les cataractes étant abaissées, il serait nécessaire de lui défendre l'usage de ses mains, jusqu'à ce qu'on eût reconnu les idées auxquelles le concours du toucher est inutile. On observerait si la lumière qu'il aperçoit lui paraît fort étendue ; s’il lui est possible d'en déterminer les bornes ; si elle est si confuse, qu'il n'y puisse pas distinguer plusieurs modifications.

Après lui avoir montré deux couleurs séparément, on les lui montrerait ensemble, et on lui demanderait s'il reconnaît quelque chose de ce qu'il a vu. Tantôt on en ferait passer successivement un plus grand nombre sous ses yeux, tantôt on les lui offrirait en même temps, et on chercherait combien il en peut démêler à-la-fois ; on examinerait surtout, s'il discerne les grandeurs, les figures, les situations, les distances et le mouvement. Mais il faudrait l'interroger avec adresse, et éviter toutes les questions, qui indiquent la réponse. Lui demander s'il voit un triangle ou un carré, ce serait lui dire comment il doit voir et donner des leçons à ses yeux. 
Moyen à employer.

Un moyen bien sûr pour faire des expériences capables de dissiper tous les doutes, ce serait d'enfermer, dans une loge de glace, l'aveugle à qui on viendrait d'abattre les cataractes. Car ou il verra les objets qui sont au-delà, et jugera de leur forme et de leur grandeur; ou il n'apercevra que l'espace borné par les côtés de sa loge, et ne prendra tous ces objets que pour des surfaces différemment colorées, qui lui paraîtront s’étendre, à mesure qu'il y portera la main.

Dans le premier cas, ce sera une preuve que l'œil juge, sans avoir tiré aucun secours du tact ; et dans le second, qu'il ne juge qu'après l’avoir consulté.

Si, comme je le présume, cet homme ne voit point au-delà de sa loge, il s'ensuit que l'espace qu'il découvre à l'œil, sera moins considérable, à mesure que sa loge sera moins grande : il sera d'un pied, d'un demi-pied, ou plus petit encore. Par là, on sera convaincu qu'il n'aurait pas pu voir les couleurs hors de ses yeux, si le toucher ne lui avait pas appris à les voir sur les côtés de sa loge. 


\section{Chapitre VII}

\section{De l'idée que la vue jointe au toucher donne de la durée}

\footnotetext{
Étonnement de la statue, la première fois qu'elle remarque le passage du jour à la nuit, et de la nuit au jour.
}

Quand notre statue commence à jouir de la lumière, elle ne sait pas encore que le soleil en est le principe. Pour en juger, il faut qu'elle ait remarqué que le jour cesse presque aussitôt que cet astre a disparu. Cet événement la surprend sans doute beaucoup, la première fois qu'il arrive. Elle croit le soleil perdu pour toujours. Environnée d'épaisses ténèbres, elle appréhende que tous les objets qu'il éclairait, ne se soient perdus avec lui : elle ose à peine changer de place, il lui semble que la terre va manquer sous ses pas. Mais au moment qu'elle cherche à la reconnaître au toucher, le ciel s'éclaircit, la lune répand sa lumière, une multitude d'étoiles brille dans le firmament. Frappée de ce spectacle, elle ne sait si elle en doit croire ses yeux.

Bientôt le silence de toute la nature l'invite au repos : un calme délicieux suspend ses sens : sa paupière s’appesantit : ses idées fuient, échappent : elle s'endort.

A son réveil, quelle est sa surprise de retrouver l'astre, qu'elle croyait s'être éteint pour jamais. Elle doute qu'il ait disparu ; et elle ne sait que penser du spectacle qui lui a succédé.

\section{Bientôt ces révolutions lui paraissent naturelles.}

Cependant, ces révolutions sont trop fréquentes, pour ne pas dissiper enfin ses doutes. Elle juge que le soleil paraîtra et disparaîtra encore, parce qu'elle a remarqué qu'il a paru et disparu plusieurs fois ; et elle porte ce jugement avec d'autant plus de confiance, qu'il a tou- 
jours été confirmé par l'événement. La succession des jours et des nuits devient donc à son égard une chose toute naturelle. Ainsi dans l'ignorance où elle est, ses idées de possibilité n’ont pour fondement que des jugements d'habitude. C'est ce que nous avons déjà observé, et ce qui ne peut manquer de l'entraîner dans bien des erreurs. Une chose, par exemple, impossible aujourd'hui, parce que le concours des causes qui peuvent seules la produire, n’a pas lieu, lui paraîtra possible, parce qu'elle est arrivée hier.

Le cours du soleil devient la mesure de sa durée.

Les révolutions du soleil attirent de plus en plus son attention. Elle l'observe lorsqu'il se levé, lorsqu'il se couche ; elle le suit dans son cours ; et elle juge à la succession de ses idées, qu'il y a un intervalle entre le lever de cet astre et son coucher, et un autre intervalle entre son coucher et son lever.

Ainsi le soleil dans sa course devient pour elle la mesure du temps, et marque la durée de tous les états par où elle passe. Auparavant une même idée, une même sensation qui ne variait point, avait beau subsister, ce n'était pour elle qu'un instant indivisible ; et quelque inégalité qu'il y eût entre les instants de sa durée, ils étaient tous égaux à son égard : ils formaient une succession, où elle ne pouvait remarquer ni lenteur, ni rapidité. Mais actuellement jugeant de sa propre durée par l'espace que le soleil a parcouru, elle lui paraît plus lente ou plus rapide. Ainsi, après avoir jugé des révolutions solaires par sa durée, elle juge de sa durée par les révolutions solaires ; et ce jugement lui devient si naturel, qu'elle ne soupçonne plus que la durée lui soit connue par la succession de ses idées.

Elle en a une idée plus distincte de la durée.

Plus elle rapportera aux différentes révolutions du soleil les évènements dont elle conserve quelque souvenir, et ceux qu'elle est accoutumée à prévoir ; plus elle en saisira toute la suite. Elle verra donc mieux dans le passé et dans l'avenir.

En effet, qu'on nous enlève toutes les mesures du temps ; n’ayons plus d'idée d'année, de mois, de jour, d'heure, oublions-en jusqu'aux noms ; alors bornés à la succession de nos idées, la durée se montrera 
à nous fort confusément. C'est donc à ces mesures, que nous en devons les idées les plus distinctes.

Dans l'étude de l'histoire, par exemple, la suite des faits retrace le temps confusément ; la division de la durée en siècles, en années, en mois, en donne une idée plus distincte ; enfin la liaison de chaque événement à son siècle, à son année, à son mois, nous rend capables de les parcourir dans leur ordre. Cet artifice consiste surtout à se faire des époques ; on conçoit que notre statue peut en avoir.

Au reste, il n'est pas nécessaire que les révolutions, pour servir de mesure, soient d'égale durée ; il suffit que la statue le suppose. Nous n'en jugeons pas nous-mêmes autrement.

Trois choses concourent à l’idée de la durée.

Trois choses concourent donc aux jugements, que nous portons sur la durée : premièrement, la succession de nos idées ; en second lieu, la connaissance des révolutions solaires ; enfin, la liaison des évènements à ces révolutions.

D'où viennent les apparences des jours longs, et des années courtes, des jours courts et des années longues.

C'est de là que naissent pour le commun des hommes les apparences des jours si longs et des années si courtes ; et pour un petit nombre les apparences des jours courts et des années longues.

Que la statue soit quelque temps dans un état, dont l'uniformité l'ennuie ; elle en remarquera davantage le temps que le soleil sera sur l'horizon, et chaque jour lui paraîtra d'une longueur insupportable. Si elle passe de la sorte une année, elle voit que tous ses jours ont été semblables, et sa mémoire n'en marquant pas la suite par une multitude d'évènements, ils lui semblent s'être écoulés avec une rapidité étonnante.

Si ses jours au contraire, passés dans un état où elle se plaît, pouvaient être chacun l'époque d'un événement singulier, elle remarquerait à peine le temps que le soleil est sur l'horizon, et elle les trouverait d'une brièveté surprenante. Mais une année lui paraîtrait longue, 
parce qu'elle se la retracerait comme la succession d'une multitude de jours distingués par une suite d'évènements.

Voilà pourquoi dans le désœuvrement nous nous plaignons de la lenteur des jours et de la rapidité des années. L’occupation au contraire fait paraître les jours courts et les années longues : les jours courts, parce que nous ne faisons pas attention au temps, dont les révolutions solaires font la mesure; les années longues, parce que nous nous les rappelons par une suite de choses, qui supposent une durée considérable. 


\section{Chapitre VIII}

\section{Comment la vue, ajoutée au toucher, donne quelque connaissance de la durée du sommeil, et apprend à distinguer l'état de songe de l'état de veille}

Comment la vue fait connaître la durée du sommeil.

Si notre statue, s'étant endormie, quand le soleil était à l'orient, se réveille, quand il descend vers l'occident, elle jugera que son sommeil a eu une certaine durée ; et si elle ne se rappelle aucun songe, elle croira avoir duré, sans avoir pensé. Mais il se pourrait que ce fût une erreur : car peut-être le sommeil n’a-t-il pas été assez profond, pour suspendre entièrement l'action des facultés de l'âme.

\section{Et fait connaître l’illusion des songes.}

Si au contraire elle se souvient d'avoir eu des songes, elle a un moyen de plus pour s'assurer de la durée de son sommeil. Mais à quoi reconnaîtra-t-elle l'illusion des songes ? A la manière frappante dont ils contredisent les connaissances qu'elle avait avant de s'endormir, et dans lesquelles elle se confirme à son réveil.

Supposez, par exemple, qu'elle ait cru, pendant le sommeil, voir des choses fort extraordinaires ; et qu'au moment où elle en va sortir, il lui parut être dans des lieux où elle n’a point encore été. Sans doute elle est étonnée de ne pas s'y trouver au réveil ; de reconnaître au contraire l'endroit où elle s'est couchée ; d'ouvrir les yeux, comme s'ils avaient été longtemps fermés à la lumière ; et de reprendre enfin l'usage de ses membres, comme si elle sortait d'un repos parfait. Elle ne sait encore, si elle s'est trompée, ou si elle se trompe. Il semble qu'elle ait également raison de croire qu'elle a changé de lieu, et qu'elle n’en a pas changé. Mais enfin ayant eu fréquemment des son- 
ges, elle y remarque un désordre, où ses idées sont toujours en contradiction avec l'état de veille qui les suit, comme avec celui qui les a précédés ; et elle juge que ce ne sont que des illusions. Car accoutumée à rapporter ses sensations hors d'elle, elle n’y trouve de la réalité, qu'autant qu'elle découvre des objets, auxquels elle les peut rapporter encore. 


\section{Chapitre IX}

De la chaîne des connaissances, des abstractions et des désirs, lorsque la vue est ajoutée au toucher, à l'ouïe et à l'odorat

Idée principale, à laquelle les sensations de la vue se lient.

Nous avons prouvé que ce sont des jugements, qui lient aux sensations de lumière et de couleur les idées d'espace, de grandeur et de figure. D'abord ces jugements se font à l'occasion des corps, qui agissent en même temps sur la vue et sur le tact : ensuite ils deviennent si familiers, que la statue les répète, lors même que l'objet ne fait impression que sur l'œil ; et elle se forme les mêmes idées que si la vue et le toucher continuaient de juger ensemble.

Par ce moyen, la lumière et les couleurs deviennent les qualités des objets ; et elles se lient à la notion de l'étendue, base de toutes les idées dont se forme la mémoire.

La chaîne des connaissances en est donc plus étendue, les combinaisons en varient davantage, et les idées interceptées occasionnent dans le sommeil mille associations différentes. Quoique dans les ténèbres, la statue verra en songe les objets éclairés de la même lumière, et peints des mêmes couleurs, qu'au grand jour.

\section{Depuis la réunion de la vue au toucher, l’idée} de sensation est plus générale.

Elle aura une notion plus générale de ce que nous appelons sensation. Car sachant que la lumière et les couleurs lui viennent par un organe particulier, elle les considérera sous ce rapport, et distinguera quatre espèces de sensations. 
Chaque couleur devient une idée abstraite.

Quand elle était bornée à la vue, une couleur n'était qu'une modification particulière de son âme. Actuellement chaque couleur devient une idée abstraite et générale ; car elle la remarque sur plusieurs corps. C'est un moyen qu'elle a de plus, pour distribuer les objets dans différentes classes.

La vue devient active.

La vue presque passive, quand elle était le seul sens de la statue, est plus active, depuis qu'elle est jointe au toucher. Car elle a appris à employer la force, qui lui a été donnée pour fixer les objets. Elle n'attend pas qu'ils agissent sur elle, elle va au-devant de leur action. En un mot, elle a appris à regarder.

Elle en est plus sensiblement le siège du désir.

Puisque l'activité de la vue augmente, elle en sera plus sensiblement le siège du désir. Nous avons vu que le désir est dans l'action des facultés, excitées par l'inquiétude que produit la privation d'un plaisir.

L'imagination s'exerce moins à retracer les couleurs.

Aussi l'imagination cessera-t-elle de retracer les couleurs avec la même vivacité ; parce que plus il est facile de se procurer les sensations mêmes, moins on s'exerce à les imaginer.

Empire des sens les uns sur les autres.

Enfin la statue capable d'attention par la vue, ainsi que par les trois autres sens, pourra se distraire des sons et des odeurs, en s'appliquant à considérer vivement un objet coloré. C'est ainsi que les sens ont les uns sur les autres le même empire, que l'imagination a sur tous. 


\section{Chapitre X}

\section{Du goût réuni au toucher}

Ce sens n'a presque pas besoin
d'apprentissage.

Le sens du goût s'instruit si promptement, qu'à peine s'aperçoit-on qu'il ait besoin d'apprentissage. Cela devait être, puisqu'il est nécessaire à notre conservation, dès les premiers moments de notre naissance.

La faim, pour la première fois, n'a point d'objet déterminé.

La faim ne peut encore avoir d'objet déterminé, lorsque la statue en éprouve pour la première fois le sentiment : car les moyens, propres à la soulager, lui sont tout-à-fait inconnus. Elle ne désire donc aucune espèce de nourriture, elle désire seulement de sortir d'un état qui lui déplaît. Dans cette vue, elle se livre à toutes les sensations agréables dont elle a connaissance. C'est le seul remède dont elle puisse faire usage, il la distrait quelque peu de sa peine.

Elle fait saisir indifféremment tout ce qui se présente.

Cependant l'inquiétude redouble, se répand dans toutes les parties de son corps, et passe d'une manière plus particulière sur ses lèvres, dans sa bouche. Alors elle porte la dent sur tout ce qui s'offre à elle, mord les pierres, la terre, broute l'herbe, et son premier choix est de se nourrir des choses qui résistent moins à ses efforts. Contente d'une nourriture qui l'a soulagée, elle ne songe pas à en chercher de meilleure. Elle ne connaît encore d'autre plaisir à manger, que celui de dissiper sa faim. 
La statue découvre des nourritures qui lui sont propres.

Mais trouvant une autre fois des fruits, dont les couleurs et les parfums charment ses sens; elle y porte la main. L'inquiétude qu'elle ressent, toutes les fois que la faim se renouvelle, lui fait naturellement saisir tous les objets qui peuvent lui plaire. Ce fruit lui reste dans les doigts : elle le fixe, elle le sent avec une attention plus vive. Sa faim augmente, elle le mord, sans en attendre d'autre bien, qu'un soulagement à sa peine. Mais quel est son ravissement ! Avec quel plaisir ne savoure-t-elle pas ces sucs délicieux ! Et peut-elle résister à l'attrait d'en manger, et d'en manger encore.

\section{Elle en fait l'objet de ses désirs.}

Ayant fait cette expérience ${ }^{33}$ à plusieurs reprises, elle se connaît un nouveau besoin, découvre par quel organe elle y peut satisfaire, et apprend quels objets y sont propres. Alors la faim n'est plus, comme auparavant, un sentiment qui n'a point d'objet déterminé : mais elle porte toutes les facultés à procurer la jouissance de tout ce qui la peut dissiper.

33 Tel est l'artifice de la nature pour nous faire apporter à nos besoins des remèdes dont nous sommes encore incapables de connaître les effets. Il se montre d'une manière admirable dans un enfant nouvellement né. L’inquiétude passe de l'estomac aux joues, à la bouche ; lui fait prendre le téton, comme il aurait saisi toute autre chose ; fait mouvoir ses lèvres de toutes sortes de manière, jusqu'à ce qu'elles aient trouvé le moyen d'exprimer le lait destiné à le nourrir. Alors l'enfant est invité par le plaisir à réitérer les mêmes mouvements ; et il fait tout ce qui nécessaire à sa conservation. 
Table des matières

\section{Chapitre X.}

\section{Observations générales sur la réunion des cinq sens}

Avec le besoin de nourriture, notre statue va devenir l'objet de bien des observations. Mais avant d'entrer dans le détail de toutes les circonstances qui y donneront lieu, il faut considérer ce qui est commun à la réunion de chaque sens avec le toucher.

\section{Idées générales que la statue se fait de ses}

sensations.

Lorsqu'elle jouit tout-à-la-fois du tact et de l'odorat, elle remarque les qualités des corps par les rapports qu'elles ont à ces deux sens, et elle se fait les idées générales des deux espèces de sensations ; sensations du toucher, sensations de l'odorat: car elle ne saurait alors confondre en une seule classe des impressions, qui se font sur des organes si différents.

Il en est de même, lorsque nous ajoutons l'ouïe, la vue et le goût à ces deux sens. Elle se connaît donc en général cinq espèces de sensations.

Si pour lors nous supposons que réfléchissant sur les corps, elle en considère les qualités, sans avoir égard aux cinq manières différentes, dont ils agissent sur ses organes ; elle aura la notion générale de sensation ; c'est-à-dire, qu'elle ne formera qu'une classe de toutes les impressions que les corps font sur elle. Et cette idée est plus générale, lorsqu'elle a trois sens, que lorsqu'elle est bornée à deux ; lorsqu'elle en a quatre, que lorsqu'elle est bornée à trois, etc. 
Comment son imagination perd de son activité.

Privée du toucher, elle était dans l'impuissance d'exercer par ellemême aucun des autres sens ; et elle ne pouvait se procurer la jouissance d'une odeur, d'un son, d'une couleur et d'une saveur, qu'autant que son imagination agissait avec une force capable de les lui rendre présentes. Mais actuellement la connaissance des corps odoriférants, sonores, palpables et savoureux, et la facilité de s'en saisir, lui sont un moyen si commode pour obtenir ce qu'elle désire, que son imagination n'a pas besoin de faire les mêmes efforts. Plus, par conséquent, ces corps seront à sa portée, moins son imagination s'exercera sur les sensations, dont ils ont donné la connaissance. Elle perdra donc de son activité : mais puisque l'odorat, l'ouïe, la vue et le goût en seront plus exercés, ils acquerront un discernement plus fin et plus étendu. Ainsi ce que ces sens gagnent par leur réunion avec le toucher, dédommage avantageusement la statue de ce qu'elle a perdu du côté de l'imagination.

Liaison de toutes les espèces de sensations dans la mémoire.

Ses sensations étant devenues à son égard les qualités mêmes des objets, elle ne peut s'en rappeler, en imaginer, ou en éprouver, qu'elle ne se représente des corps. Par-là elles entrent toutes dans quelquesunes des collections que le tact lui a fait faire, deviennent des propriétés de l'étendue, se lient étroitement à la chaîne des connaissances par la même idée fondamentale, que les sensations du toucher ; et la mémoire, ainsi que l'imagination, en sont plus riches, que lorsqu'elle n’avait pas encore l'usage de tous ses sens.

Activité qu'acquiert la statue par la réunion du toucher aux autres sens.

Nous avons remarqué, quand nous considérions l'odorat, l'ouïe, la vue et le goût, chacun séparément, que notre statue était toute passive par rapport aux impressions qu'ils lui transmettaient. Mais actuellement elle peut être active à cet égard dans bien des occasions : car elle a en elle des moyens pour se livrer à l'impression des corps, ou pour s'y soustraire. 
Comment ses désirs embrassent l'action de toutes les facultés.

Nous avons aussi remarqué, que le désir ne consistait que dans l'action des facultés de l'âme, qui se portaient à une odeur, dont il restait quelque souvenir. Mais depuis la réunion de l'odorat au toucher, il peut encore embrasser l'action de toutes les facultés propres à lui procurer la jouissance d'un corps odoriférant. Ainsi lorsqu'elle désire une fleur, le mouvement passe de l'organe de l'odorat dans toutes les parties du corps ; et son désir devient l'action de toutes les facultés dont elle est capable.

Il faut remarquer la même chose à l'occasion des autres sens. Car le toucher les ayant instruit, continue d'agir avec eux, toutes les fois qu'il peut leur être de quelque secours. Il prend part à tout ce qui les intéresse ; leur apprend à s’aider tous réciproquement ; et c'est à lui que tous nos organes, toutes nos facultés doivent l'habitude de se porter vers les objets propres à notre conservation. 


\section{QUATRIÈME PARTIE}

\section{Des besoins, de l'industrie et des idées d'un homme isolé qui jouit de tous ses sens}

Table des matières

Si on se rappelle que j’ai démontré combien les signes sont nécessaires pour se faire des idées distinctes de toute espèce, on sera porté à juger que je suppose souvent dans la Statue plus de connaissance qu'elle n’en peut acquérir.

Mais il faut distinguer, comme j'ai fait plus haut, des connaissances de théorie et des connaissances pratiques. Or ce sont les premières pour lesquelles nous avons besoin d'un langage, parce qu'elles consistent dans une suite d'idées distinctes, et que par conséquent il a fallu des signes pour les classer avec ordre et les déterminer.

Les connaissances pratiques sont au contraire des idées confuses, qui règlent nos actions sans que nous soyons capables de remarquer comment elles nous font agir. C'est qu'elles consistent plutôt dans les habitudes qui sont une suite de nos jugements, que dans nos jugements mêmes. Car, lorsqu'une fois nous avons contracté ces habitudes, nous agissons sans pouvoir observer les jugements qui les accompagnent, et c'est pourquoi nous ne pouvons pas nous en rendre compte. Alors quoique nous nous conduisions bien, c'est sans savoir comment, à notre insu ; et nous obéissons à une impulsion, à un instinct que nous ne connaissons pas : car ces mots impulsion et instinct signifient proprement la même chose.

Il y a longtemps qu’on est forcé de reconnaître qu'il entre nécessairement des jugements dans l'usage que nous faisons de nos sens. Quand donc j'aurais mal expliqué comment la statue apprend à se ser- 
vir des siens, il n'en serait pas moins vrai qu'elle porte des jugements. Or ces jugements, qu'elle ne remarque pas, sont l'instinct qui la conduit ; et les habitudes d'agir qu'elle a contractées d'après ces jugements, sont ce que j'entends par connaissances pratiques. Si pour faire connaître ces jugements, je suis obligé de les développer, je ne prétends pas qu'elle les développe elle-même. Elle ne le peut pas, parce que n'ayant point de langage, elle n'a pas de moyens pour en faire l'analyse. Mais pour contracter des habitudes, il lui suffit de porter ces jugements, et elle n'a pas besoin de les remarquer. Croira-t-on qu'un enfant ne commence à juger que lorsqu'il commence à parler ? Certainement il ne sentirait pas le besoin d'apprendre une langue, s'il ne sentait pas celui de prononcer des jugements. Il en a donc déjà porté, quand il commence à parler, c'est-à-dire, quand il commence à faire, avec des mots, l'analyse de sa pensée : il ne dit que ce qu'il faisait auparavant sans pouvoir le dire. 
Table des matières

\section{Chapitre premier}

\section{Comment cet homme apprend à satisfaire à ses besoins avec choix}

\section{La statue sans besoin.}

Si nous imaginons que la nature dispose les choses de manière à prévenir tous les besoins de notre statue, et que voulant la toucher avec les précautions d'une mère qui craint de blesser ses enfants, elle en écarte jusqu'aux plus légères inquiétudes, et se réserve à elle seule le soin de veiller à sa conservation ; cet état nous paraîtra peut-être digne d'envie. Néanmoins que serait-ce qu'un homme de cette espèce ? Un animal enseveli dans une profonde léthargie. Il est, mais il reste comme il est ; à peine se sent-il. Incapable de remarquer les objets qui l'environnent, incapable d'observer ce qui se passe en luimême ; son âme se partage indifféremment entre toutes les perceptions, auxquelles ses sens ouvrent un passage. En quelque sorte semblable à une glace, sans cesse il reçoit de nouvelles images, et jamais il n'en conserve aucune.

En effet, quelle occasion aurait cet homme de s'occuper de lui, ou de ce qui est au-dehors ? La nature a tout pris sur elle, et elle a si fort prévenu ses besoins, qu'elle ne lui laisse rien à désirer. Elle a voulu éloigner de lui toute inquiétude, toute douleur : mais pour avoir craint de le rendre malheureux, elle le borne à des sensations, dont il ne peut connaître le prix, et qui passent comme une ombre.

Avec des besoins faciles à satisfaire.

J'exige donc qu'elle paraisse moins occupée du soin de prévenir les maux dont il peut être menacé ; qu'elle s'en repose quelque peu sur lui ; et qu'elle se contente de mettre à sa portée toutes les choses nécessaires à ses besoins. 
Dans cette abondance, la statue forme des désirs ; mais elle a dans le moment toujours de quoi se satisfaire. Toute la nature semble encore veiller sur elle : à peine a-t-elle permis que son repos fût interrompu par le moindre malaise, qu'elle paraît s'en repentir, et qu'elle donne tous ses soins à prévenir une plus grande inquiétude. Par cette vigilance, elle la met à l'abri de bien des maux, mais aussi elle la frustre de bien des plaisirs. Le malaise est léger, le désir qui le suit est peu de chose, la prompte jouissance ne permet pas qu'aucun besoin augmente considérablement, et le plaisir, qui en fait tout le prix, est proportionné à la faiblesse du besoin.

Le repos de notre statue étant aussi peu troublé, l'équilibre s'entretient presque toujours également dans toutes les parties de son corps, et son tempérament souffre à peine quelque altération. Elle doit, par conséquent, se conserver longtemps : mais elle vit dans un degré bien faible, et qui n'ajoute à l'existence que le moins qu'il est possible.

Difficiles à satisfaire.

Changeons la scène, et supposons que la statue ait des obstacles à surmonter, pour obtenir la possession de ce qu'elle désire. Alors les besoins subsistent longtemps avant d'être soulagés. Le malaise, faible dans son origine, devient insensiblement plus vif ; il se change en inquiétude, il se termine quelquefois à la douleur.

Tant que l'inquiétude est légère, le désir a peu de force : la statue se sent peu pressée de jouir : une sensation vive peut la distraire et suspendre sa peine. Mais le désir augmente avec l'inquiétude ; il vient un moment, où il agit avec tant de violence, qu'on ne trouve de remède que dans la jouissance : il se change en passion.

\section{La statue encore sans prévoyance.}

La première fois que la statue satisfait à un besoin, elle ne devine pas qu'elle doive l'éprouver encore. Le besoin soulagé, elle s’abandonne à sa première tranquillité.

Ainsi, sans précaution pour l'avenir, elle ne songe qu'au présent, elle ne songe qu'à écarter la peine que produit un besoin, au moment qu'elle souffre. 
Comment elle en devient capable.

Elle demeure à-peu-près dans cet état, tant que ses besoins sont faibles, en petit nombre, et qu'elle trouve peu d'obstacles à les soulager. Accoutumée à régler ses désirs sur l'intérêt, qui naît du contraste des plaisirs et des peines, il n'y a que l'expérience des maux qu'elle souffre, pour ne les avoir pas prévus, qui puisse lui faire porter ses vues au-delà de sa situation présente. Le passé peut seul lui apprendre à lire dans l'avenir.

Elle ne peut donc remarquer la fréquence de ses besoins, et les tourments qu'elle a essuyés, toutes les fois qu'elle n'a pas eu assez tôt de quoi y remédier, qu'elle ne se fasse bientôt une habitude de les prévoir, et de prendre des précautions pour les prévenir, ou pour les soulager de bonne heure. Dans le temps même, où elle n'a pas le moindre malaise, l'imagination lui rappelle tous les maux auxquels elle a été exposée, et les lui représente comme prêts à l'accabler encore. Aussitôt elle ressent une inquiétude de la même espèce que celle que le besoin pourrait produire ; elle souffre d'avance quelque chose de semblable à ce qu'elle souffrirait, si le besoin était présent.

Combien l'imagination ne la rendrait-elle pas malheureuse, si elle bornait là ses effets ! Mais elle lui retrace bientôt les objets, qui ont servi plusieurs fois à la soulager. Dès-lors elle lui fait presque goûter les mêmes plaisirs que la jouissance ; et l'on dirait qu'elle ne lui a donné de l'inquiétude, pour un mal éloigné, qu'afin de lui procurer une jouissance qui anticipe sur l'avenir.

Ainsi, tandis que la crainte la menace de maux semblables à ceux qu'elle a déjà soufferts, l'espérance la flatte de les prévenir, ou d'y remédier : l'une et l'autre lui dérobent à l'envi le sentiment du moment présent pour l'occuper d'un temps, qui n'est point encore, ou qui même ne sera jamais ; et de ces deux passions naissent le besoin de précautions, et l'adresse à en prendre. Elle passe donc, tour-à-tour de l'une à l'autre, suivant que les dangers se répètent, et qu'ils sont plus ou moins difficiles à éviter ; et ces passions acquièrent tous les jours de nouvelles forces. Elle s'effraie ou se flatte à tous propos. Dans l'espérance, l'imagination lui levé tous les obstacles, lui présente les objets par les plus beaux côtés, et lui fait croire qu'elle en va jouir : illusion qui souvent la rend plus heureuse, que la jouissance. Dans la 
crainte, elle voit tous les maux ensemble, elle en est menacée, elle touche au moment où elle en doit être accablée, elle ne connaît aucun moyen de les éviter, et peut-être serait-elle moins malheureuse de les ressentir.

C'est ainsi que l'imagination lui présente tous les objets, qui ont quelque rapport à l'espérance ou à la crainte. Tantôt l'une de ses passions domine, tantôt l'autre ; et quelquefois elles se balancent si bien, qu'on ne saurait déterminer laquelle des deux agit davantage. Destinées à rendre la statue plus industrieuse sur les mesures nécessaires à sa conservation, elles paraissent veiller à ce qu'elle ne soit ni trop heureuse, ni trop malheureuse.

Progrès de sa raison à cet égard.

Instruite, par l'expérience des moyens qui peuvent soulager ou prévenir ses besoins, elle réfléchit sur les choix qu'elle a à faire. Elle examine les avantages et les inconvénients des objets qu'elle a jusqu'à présent fuis ou recherchés. Elle se rappelle les méprises où elle est tombée, pour s'être souvent déterminée trop à la hâte, et avoir obéi aveuglément au premier mouvement de ses passions. Elle regrette de ne s'être pas mieux conduite. Elle sent que désormais, il dépend d'elle de se régler d'après les connaissances qu'elle a acquises: et s'accoutumant à en faire usage, elle apprend peu-à-peu à résister à ses désirs, et même à les vaincre. C'est ainsi qu'intéressée à éviter la douleur, elle diminue l'empire des passions, pour étendre celui que la raison doit avoir sur sa volonté, et pour devenir libre ${ }^{34}$.

L’ordre de ses études est déterminé par ses

besoins.

Dans cette situation, elle étudie d'autant plus les objets, qui peuvent contribuer à ses plaisirs ou à ses peines, qu'elle sait avoir souffert, pour ne les avoir pas assez connus ; et que l'expérience lui prouve qu'il est à sa disposition de les mieux connaître. Ainsi l'ordre de ses études est déterminé par ses besoins. Les plus vifs et les plus fréquents sont donc ceux qui l'engagent dans les premières recherches qu'elle fait.

34 Voyez la Dissertation qui est à la fin de cet ouvrage. 
Et principalement par le besoin de nourriture.

Tel est le besoin de nourriture, comme plus nécessaire à sa conservation. En soulageant sa faim, elle renouvelle ses forces; et elle sent qu'il lui est important de les renouveler, pour jouir de toutes ses facultés. Tous ses autres besoins cèdent à celui-là. La vue, le toucher, l'ouïe et l'odorat ne semblent faits que pour découvrir et procurer ce qui peut flatter le goût. Elle prend donc un nouvel intérêt à tout ce que la nature offre à ses regards. Sa curiosité ne se borne plus à démêler la couleur des objets, leur odeur, leur figure, etc. Si elle les étudie par ces qualités, c'est surtout pour apprendre à reconnaître ceux qui sont propres à la nourrir. Elle ne voit donc point un fruit, dont elle a mangé, elle ne le touche point, elle ne le sent point ; sans juger s'il est bon ou mauvais au goût. Ce jugement augmente le plaisir qu'elle a de le voir, de le toucher, de le sentir ; et ce sens contribue à lui rendre les autres d'un plus grand prix. Il a surtout beaucoup d'analogie avec l'odorat. Le parfum des fruits l'intéressait bien moins, avant qu'elle eût l'organe du goût ; et le goût perdrait toute sa finesse, si elle était privée de l'odorat. Mais dès qu'elle a ces deux sens, leurs sensations se confondent, et en deviennent plus délicieuses.

Elle donne à ses idées un ordre bien différent de celui qu'elles avaient auparavant ; parce que le besoin qui détermine ses facultés, est lui-même bien différent de ceux qui l'ont mue jusqu'alors. Elle s'applique avec intérêt à des objets, auxquels elle n’avait point encore donné d'attention; et ceux dont elle peut se nourrir, sont aussi ceux qu'elle distingue en plus de classes. Elle s'en fait des idées complexes, en les considérant comme ayant telle couleur, telle odeur, telle forme et telle saveur à-la-fois ; et elle se forme à leur occasion des idées abstraites et générales, en considérant les qualités qui sont communes à plusieurs.

Jugements qui donnent plus d'étendue à ce besoin.

Elle les compare les uns avec les autres, et elle désire d'abord de se nourrir par préférence de ces fruits, où elle se souvient d'avoir trouvé un goût, qui lui a plu davantage. Dans la suite elle s'accoutume peu-àpeu à cette nourriture ; et l'habitude qu'elle s'en fait, devient quelque- 
fois si grande, qu'elle influe autant dans son choix, que le plaisir même.

Elle mêle donc bientôt des jugements au plaisir qu'elle trouve à en faire usage. Si elle n'en mêlait pas, elle ne serait portée à manger que pour se nourrir. Mais ce jugement, il est bon, il est excellent, il est meilleur que tout autre, lui fait un besoin de la sensation qu'un fruit peut produire. Ce qui suffit alors à la nourrir, ne suffit pas à son plaisir. Il y a en elle deux besoins, l'un causé par la privation de nourriture, l'autre par la privation d'une saveur qui mérite la préférence ; et ce dernier est une faim, qui la trompe quelquefois, et qui la fait manger au-delà du nécessaire.

Excès où tombe la statue.

Cependant son goût se blase pour certains fruits : alors, ou elle s'en dégoûte tout-à-fait, ou si elle désire encore d'en manger, ce n'est plus que par habitude. Dans ce dernier cas, elle s'en nourrit, en espérant toujours de le savourer, comme elle a fait auparavant. Elle y est si fort accoutumée, qu'elle s'imagine toujours qu'elle va retrouver un plaisir, pour lequel elle n'est plus faite ; et cette idée contribue à entretenir son désir.

Frustrée dans son espérance, son désir n'en devient que plus violent. Elle fait de nouveaux essais, et elle en fait jusqu'à ce qu'il ne lui soit plus possible de continuer. C'est ainsi que les excès où elle tombe, ont souvent pour cause une habitude contractée, et l'ombre d'un plaisir que l'imagination lui retrace sans cesse, et qui lui échappe toujours.

\section{Elle en est punie.}

Elle en est punie. La douleur l'avertit bientôt que le but du plaisir n'est pas uniquement de la rendre heureuse pour le moment, mais encore de concourir à sa conservation ; ou plutôt de rétablir ses forces, pour lui rendre l'usage de ses facultés : car elle ne sait pas ce que c'est que se conserver. 


\section{Combien il était nécessaire de l’avertir par la} douleur.

Si la nature, par affection pour elle, n'eût attaché à ces effets que des sentiments agréables, elle l'eût trompée et se fût trompée ellemême : la statue, croyant chercher son bonheur, n'eût couru qu'à sa perte.

Mais ces avertissements ne peuvent se répéter, qu'elle n'apprenne enfin qu'elle doit mettre un frein à ses désirs. Car rien n'est si naturel que de regarder, comme l'effet d'une chose, ce qui vient constamment à sa suite.

Dès-lors, elle n’éprouvera plus de pareils désirs, que l'imagination ne lui retrace aussitôt tous les maux qu'elle a soufferts. Cette vue lui fait craindre jusqu'aux objets qui lui plaisent davantage ; et elle est entre deux inquiétudes qui se combattent.

Si l'idée des peines se réveille avec peu de vivacité, la crainte sera faible, et ne fera que peu de résistance. Si elle est vive, la crainte sera forte, et tiendra plus longtemps en suspens. Enfin cette idée pourra être à un point, où éteignant tout-à-fait le désir, elle inspirera du dégoût pour un objet, qui avait été souhaité avec ardeur.

C'est ainsi, que voyant, tout-à-la-fois du plaisir et du danger, à préférer les fruits qu'elle aime davantage, elle apprendra à se nourrir avec plus de choix ; et que trouvant plus d'obstacles à satisfaire ses désirs, elle en sera exposée à des besoins plus grands. Car ce n'est pas assez qu'elle remédie à l'inquiétude causée par le besoin de nourriture, il faut encore qu'elle apaise l'inquiétude que produit la privation d'un plaisir, et qu'elle l'apaise sans danger. 
Table des matières

\section{Chapitre II}

De l'état d'un homme abandonné à lui-même, et comment les accidents auxquels il est exposé, contribuent à son instruction

Circonstances où la statue ne se borne pas à

l'étude des objets propres à la nourrir.

La statue étant instruite des objets propres à la nourrir, sera plus ou moins occupée du soin de sa nourriture, suivant les obstacles qu'elle aura à surmonter. Ainsi nous pouvons la supposer dans un séjour, où toute entière à ce besoin, elle n'acquerrait point d'autres connaissances.

Si nous diminuons les obstacles, elle sera aussitôt appelée par les plaisirs qui s'offrent à chacun de ses sens. Elle s'intéressera à tout ce qui les frappe. Par conséquent, tout entretiendra sa curiosité, l'excitera, l'augmentera ; et elle passera tour-à-tour de l'étude des objets propres à la nourrir, à l'étude de tout ce qui l'environne.

Elle s'étudie.

Tantôt la curiosité la porte à s’étudier elle-même. Elle observe ses sens, les impressions qu'ils lui transmettent ; ses plaisirs, ses peines, ses besoins, les moyens de les satisfaire ; et elle se fait une espèce de plan de ce qu'elle a à fuir ou à rechercher.

Elle étudie les objets.

D’autres fois elle étudie plus particulièrement les objets qui attirent son attention. Elle en fait différentes classes, suivant les différences qu'elle y remarque ; et le nombre de ses notions abstraites augmente, à proportion que sa curiosité est excitée par le plaisir de voir, de sentir, de goûter, d'entendre, de toucher. 
La curiosité lui fait-elle porter les yeux sur les animaux, elle voit qu'ils se meuvent et se nourrissent, comme elle ; qu'ils ont des organes, pour saisir ce qui leur convient ; des yeux, pour se conduire ; des armes, pour attaquer, ou pour se défendre ; de l'agilité ou de l'adresse, pour échapper au danger ; de l'industrie, pour tendre des pièges : et elle les distingue par la figure, les couleurs, et surtout par les qualités qui l'étonnent davantage.

Surprise des combats qu'ils se livrent, elle l'est bien plus encore, lorsqu'elle remarque que les plus faibles déchirés par les plus forts, répandent leur sang, et perdent tout mouvement. Cette vue lui peint sensiblement le passage de la vie à la mort : mais elle ne pense pas qu'elle puisse être destinée à finir de la même manière. La vie lui paraît une chose si naturelle, qu'elle n'imagine pas comment elle en pourrait être privée. Elle sait seulement qu'elle est exposée à la douleur; qu'il y a des corps, qui peuvent l'offenser, la déchirer. Mais l'expérience lui a appris à les connaître et à les éviter.

Elle vit donc dans la plus grande sécurité, au milieu des animaux qui se font la guerre. L'univers est un théâtre où elle n'est que spectateur; et elle ne prévoit pas qu'elle en doive jamais ensanglanter la scène.

\section{Accidents auxquels elle est exposée.}

Cependant un ennemi vient à elle. Ignorant le péril qui la menace, elle ne songe point à l'éviter, et elle en fait une cruelle expérience. Elle se défend. Heureusement assez forte pour se soustraire à une partie des coups qui lui sont portés, elle échappe : elle n’a reçu que des blessures peu dangereuses. Mais l'idée de cet animal reste présente à sa mémoire ; elle se lie à toutes les circonstances, où elle en a été assaillie. Est-ce dans un bois ? La vue d'un arbre, le bruit des feuilles mettra sous ses yeux l'image du danger. Elle a une vive frayeur, parce qu'elle est faible ; elle la sent se renouveler, parce qu'elle ignore encore les précautions que sa situation demande ; tout devient pour elle un objet de terreur, parce que l'idée du péril est si fort liée à tout ce qu'elle rencontre, qu'elle ne sait plus discerner ce qu'elle doit craindre. Un mouton l'épouvante ; et, pour oser l'attendre, il lui faudrait un courage qu'elle ne peut encore avoir. 
Revenue de son premier trouble, elle est presque étonnée de voir des animaux qui fuient devant elle. Elle les voit fuir encore, et elle s'assure enfin qu'elle n'en a rien à craindre.

A peine commence-t-elle à secouer son inquiétude, que son premier ennemi reparaît, ou qu'elle est même attaquée par un autre. Elle échappe encore, non sans en avoir reçu quelque offense.

\section{Comment elle apprend à s'en garantir.}

Ces sortes d'accidents l'inquiètent, la troublent à proportion qu'ils se multiplient davantage, et que les suites en sont plus fâcheuses. La frayeur qu'elle en a, occasionne dans toutes les parties de son corps de violents frémissements. Les dangers passent ; mais les frémissements durent, ou se renouvellent à chaque instant, et en retracent l'image. Incapable de faire la différence des circonstances, suivant qu'il est plus ou moins probable qu'elle est à l'abri de pareils événements, elle a la même inquiétude pour un péril éloigné, et pour celui qui la menace de près : souvent même elle en a une plus grande. Elle les fuit également tous deux ; parce qu'elle sent toute sa faiblesse, quand elle a attendu trop tard, pour se garantir. Ainsi sa crainte devenant plus active que son espérance, elle en suit davantage les mouvements : et elle prend bien plus de précautions contre les maux auxquels elle est exposée, que de mesures pour obtenir les biens dont elle peut jouir. Elle s'applique donc à reconnaître les animaux, qui lui font la guerre ; elle fuit les lieux qu'ils paraissent habiter : elle juge de ce qu'elle en a à craindre par les coups qu'elle leur voit porter à ceux qui sont faibles comme elle. La frayeur de ces derniers redouble la sienne ; leur fuite, leurs cris l'avertissent du danger qui la menace. Tantôt elle s'étudie à l'éviter par adresse : tantôt elle se saisit pour sa défense de tout ce que le hasard lui présente ; supplée par industrie, mais avec bien de la lenteur, aux armes que la nature lui a refusées ; apprend peu-à-peu à se défendre ; sort victorieuse du combat; et flattée de ses succès, elle commence à se sentir un courage qui la met quelquefois au-dessus du péril, ou qui même, la rend téméraire. Alors tout prend pour elle une face nouvelle; elle a de nouvelles vues, de nouveaux intérêts : sa curiosité change d'objets ; et souvent plus occupée de sa défense que du besoin de nourriture, elle ne s'applique qu'à combattre avec avantage. 
Autres accidents.

Elle est bientôt exposée à de nouveaux maux. La saison change presque tout-à-coup, les plantes se dessèchent, le pays devient aride, et elle respire un air qui la blesse de toute part ; elle apprend à se vêtir de tout ce qui peut entretenir sa chaleur, et à se réfugier dans les lieux où elle est plus à l'abri des injures du ciel.

Cependant souvent exposée à souffrir longtemps par la privation de toute sorte de nourriture, c'est alors qu'elle use de la supériorité que l'adresse ou la force lui donne sur quelques animaux : elle les attaque, les saisit, les dévore. N'ayant plus d'autre moyen pour se nourrir, elle imagine des ruses, des armes : elle réussit d'autant plus dans cet art, que le combat lui devient aussi essentiel que la nourriture. La voilà donc en guerre avec tous les animaux, soit pour attaquer, soit pour se défendre.

C'est ainsi que l'expérience lui donne des leçons, qu'elle lui fait souvent payer de son sang. Mais pouvait-elle l'instruire à moins de frais ?

Conclusion.

Se nourrir, se précautionner contre tout accident, ou s'en défendre et satisfaire sa curiosité : voilà tous les besoins naturels de notre statue. Ils déterminent tour-à-tour ses facultés, et ils sont le principe des connaissances qu'elle acquiert. Tantôt supérieure aux circonstances, elle ouvre une libre carrière à ses désirs ; d'autres fois subjuguée par les circonstances, elle trame elle-même ses malheurs. Si les succès sont traversés par des revers, les revers sont aussi réparés par des succès ; et les objets semblent tour-à-tour conspirer à ses peines et à ses plaisirs. Elle flotte donc entre la confiance et l'incertitude, et traînant ses espérances et ses craintes, elle touche d'un moment à l'autre à son bonheur et à sa ruine. L'expérience seule la met insensiblement audessus des dangers, l'élève aux connaissances nécessaires à sa conservation, et lui fait contracter toutes les habitudes qui la doivent gouverner. Mais comme sans expérience, il n'y aurait point de connaissances ; il n’y aurait point d'expérience sans les besoins, et il n’y aurait point de besoins sans l'alternative des plaisirs et des peines. Tout est 
donc le fruit du principe que nous avons établi, dès l'entrée de cet ouvrage.

Nous allons traiter des jugements que la statue porte des objets, suivant la part qu'ils ont à ses plaisirs ou à ses peines. 


\section{Chapitre III}

\section{Des jugements qu'un homme abandonné à lui-même peut porter de la bonté et de la beauté des choses}

Définition des mots bonté et beauté.

Les mots bonté et beauté, expriment les qualités par où les choses contribuent à nos plaisirs. Par conséquent, tout être sensible a des idées d'une bonté et d'une beauté relatives à lui.

En effet on appelle bon, tout ce qui plaît à l'odorat ou au goût ; et on appelle beau, tout ce qui plaît à la vue, à l'ouïe ou au toucher.

Le bon et le beau sont encore relatifs aux passions et à l'esprit. Ce qui flatte les passions est bon ; ce que l'esprit goûte est beau ; et ce qui plaît en même temps aux passions et à l'esprit, est bon et beau tout ensemble.

\section{La statue a des idées du bon et du beau.}

Notre statue connaît des odeurs et des saveurs agréables, et des objets qui flattent ses passions: elle a donc des idées du bon. Elle connaît aussi des objets qu'elle voit, qu'elle entend, qu'elle touche, et que son esprit conçoit avec plaisir : elle a donc encore des idées du beau.

\section{Le bon et le beau ne sont pas absolus.}

Une conséquence qui se présente, c'est que le bon et le beau ne sont point absolus : ils sont relatifs au caractère de celui qui en juge, et à la manière dont il est organisé ${ }^{35}$.

35 Il ne faut pas perdre de vue le titre de ce chapitre. Nous considérons un homme qui vit seul, et nous ne cherchons pas quelle est la bonté et la beauté des 
Ils se prêtent mutuellement des secours.

Le bon et le beau se prêtent des secours mutuels. Une pêche que voit la statue, lui plaît par la vivacité des couleurs : elle est belle à ses yeux. Aussitôt la saveur s'en retrace à son imagination : elle est vue avec plus de plaisir, elle en est plus belle.

La statue mange cette pêche ; alors le plaisir de la voir se mêle à celui de la goûter : elle en est meilleure.

L’utilité contribue à l'un et à l'autre.

L'utilité contribue à la bonté et à la beauté des choses. Les fruits bons et beaux, par le seul plaisir de les voir et de les savourer, sont meilleurs et plus beaux, lorsque nous pensons qu'ils sont propres à rétablir nos forces.

La nouveauté et la rareté y contribuent aussi.

La nouveauté et la rareté y contribuent aussi : car l'étonnement que donne un objet déjà bon et beau par lui-même, joint à la difficulté de le posséder, augmente le plaisir d'en jouir.

Deux sortes de bontés et de beautés.

La bonté et la beauté des choses consistent dans une seule idée, ou dans une multitude d'idées qui ont certains rapports entre elles. Une seule saveur, une seule odeur peuvent être bonnes : la lumière est belle, un son pris tout seul peut être beau.

Mais lorsqu'il y a multitude d'idées, un objet est meilleur ou plus beau, à proportion que les idées se démêlent davantage, et que leurs rapports sont aperçus : car on jouit avec plus de plaisir. Un fruit où l'on reconnaît plusieurs saveurs, également agréables, est meilleur qu'une seule de ces saveurs : un objet dont les couleurs se prêtent mutuellement de l'éclat, est plus beau que la lumière seule.

choses, nous cherchons seulement les jugements qu'il en peut porter. Tout ce qu'il jugera bon, ne sera pas moralement bon ; comme tout ce qu'il jugera beau, ne sera pas réellement beau. 
Les organes ne peuvent saisir distinctement qu'un certain nombre de sensations ; l'esprit ne peut comparer à-la-fois qu'un certain nombre d'idées : une trop grande multitude fait confusion. Elle nuit donc au plaisir, et par conséquent à la bonté et à la beauté des choses.

Une petite quantité de sensations ou d'idées se confondent encore si quelqu'une domine trop sur les autres. Il faut donc pour la plus grande bonté et pour la plus grande beauté, que le mélange en soit fait suivant certaines proportions.

\section{Comment la statue y est sensible.}

C'est à l'exercice de ses organes et de son esprit, que notre statue doit l'avantage d'embrasser plus d'idées et plus de rapports. Le bon et le beau sont donc encore relatifs à l'usage qu'elle a appris à faire de ses facultés. Telle chose qui dans un temps, a été fort bonne ou fort belle, cessera de l'être ; tandis qu'une autre à laquelle elle n'avait donné aucune attention, deviendra de la plus grande bonté ou de la plus grande beauté.

En cela, comme en toute autre chose, elle ne jugera que par rapport à elle. D'abord, elle prend ses modèles dans les objets qui contribuent plus directement à son bonheur ; ensuite elle juge des autres objets par ces modèles, et ils lui paraissent plus beaux, lorsqu'ils leur ressemblent davantage. Car après cette comparaison, elle trouve à les voir un plaisir qu'elle n'avait point goûté jusqu'alors. Un arbre, par exemple, chargé de fruits, lui plaît et lui rend agréable la vue d'un autre qui n'en porte point, mais qui a quelque ressemblance avec lui.

Pourquoi elle a à ce sujet moins d'idées que

nous.

Il n'est pas possible d'imaginer tous les différents jugements qu'elle portera suivant les circonstances : ce serait d'ailleurs une recherche assez inutile. Il suffit d'observer qu'il y a pour elle, comme pour nous, une bonté et une beauté réelles ou arbitraires ; et que si elle a à ce sujet moins d'idées, c'est qu'aussi elle a moins de besoins, moins de connaissances et moins de passions. 


\section{Chapitre IV \\ Des jugements qu'un homme abandonné à lui-même peut porter des objets dont il dépend}

La statue croit que tout ce qui agit sur elle, agit avec dessein.

La statue sent à chaque instant la dépendance, où elle est de tout ce qui l'environne. Si les objets répondent souvent à ses vœux, ils traversent presque aussi souvent ses projets : ils la rendent malheureuse, ou ne lui accordent qu’une partie du bonheur qu'elle désire.

Persuadée qu'elle ne fait rien, sans avoir intention de le faire ; elle croit voir un dessein, partout où elle découvre quelque action. En effet, elle n'en peut juger que d'après ce qu'elle remarque en ellemême ; et il lui faudrait bien des observations, pour parvenir à mieux régler ses jugements. Elle pense donc que ce qui lui plaît, a en vue de lui plaire ; et que ce qui l'offense, a en vue de l'offenser. Par là, son amour et sa haine deviennent des passions d'autant plus violentes, que le dessein de contribuer à son bonheur ou à son malheur, se montre plus sensiblement dans tout ce qui agit sur elle.

\section{Superstitions, où ce préjugé l'entraîne.}

Alors elle ne se borne plus à désirer la jouissance des plaisirs, que les objets peuvent lui procurer ; et l'éloignement des peines dont ils la menacent : elle souhaite qu'ils aient intention de la combler de biens, et de détourner de dessus sa tête toute sorte de maux : elle souhaite en un mot qu'ils lui soient favorables, et ce désir est une sorte de prière.

Elle s'adresse en quelque sorte au soleil ; et parce qu'elle juge que s’il l'éclaire et l'échauffe, il a dessein de l'éclairer et de l'échauffer, elle le prie de l'éclairer et de l'échauffer encore. Elle s'adresse aux arbres, et elle leur demande des fruits, ne doutant pas qu'il dépend 
d'eux d'en porter ou de n'en pas porter. En un mot, elle s'adresse à toutes les choses dont elle croit dépendre.

Souffre-t-elle sans en découvrir la cause dans ce qui frappe ses sens ? Elle s'adresse à la douleur, comme à un ennemi invisible, qu'il lui est important d'apaiser. Ainsi l'univers se remplit d'êtres visibles et invisibles, qu'elle prie de travailler à son bonheur.

Telles sont ses premières idées, lorsqu'elle commence à réfléchir sur sa dépendance. D'autres circonstances donneront lieu à d'autres jugements, et multiplieront ses erreurs. J'ai fait voir ailleurs ${ }^{36}$ les égarements, où l'on peut être entraîné par la superstition : mais je renvoie aux ouvrages des philosophes éclairés, pour s'instruire des découvertes que la raison bien conduite peut faire à ce sujet.

36 Traité des Systèmes, ch. de la Divination. 


\section{Chapitre V \\ De l'incertitude des jugements que nous portons sur l'existence des qualités sensibles}

Nos jugements sur l'existence des qualités sensibles, pourraient absolument être faux.

Notre statue, je le suppose, se souvient qu'elle a été elle-même son, saveur, odeur, couleur : elle sait combien elle a eu de peine à s'accoutumer à rapporter ces sensations au-dehors. Y a-t-il donc dans les objets des sons, des saveurs, des odeurs, des couleurs ? Qui peut l'en assurer ? Ce n'est certainement ni l'ouïe, ni l'odorat, ni le goût, ni la vue : ces sens par eux-mêmes ne peuvent l'instruire que des modifications qu'elle éprouve. Elle n'a d'abord senti que son être, dans les impressions dont ils sont susceptibles ; et s'ils les lui font aujourd'hui sentir dans les corps, c'est qu'ils ont contracté l'habitude de juger d'après le témoignage du tact. Y a-t-il donc au moins de l'étendue ? Mais lorsqu'elle a le sentiment du toucher, qu'aperçoit-elle si ce n'est encore ses propres modifications? Le toucher n'est donc pas plus croyable que les autres sens : et puisqu'on reconnaît que les sons, les saveurs, les odeurs et les couleurs n'existent pas dans les objets, il se pourrait que l'étendue n'y existât pas davantage ${ }^{37}$.

S’il n’y a point d'étendue, dira-t-on peut-être, il n'y a point de corps. Je ne dis pas qu'il n'y a point d'étendue, je dis seulement que nous ne l'apercevons que dans nos propres sensations. D’où il s'ensuit que nous ne voyons point les corps en eux-mêmes. Peut-être sont-ils étendus, et même savoureux, sonores, colorés, odoriférants : peut-être ne sont-ils rien de tout cela. Je ne soutiens ni l'un ni l'autre ; et j'attends qu'on ait prouvé qu'ils sont ce qu'ils nous paraissent, ou qu'ils sont toute autre chose.

N'y eût-il point d'étendue, ce ne serait donc pas une raison pour nier l'existence des corps. Tout ce qu'on pourrait et devrait raisonnablement inférer, c'est que les corps sont des êtres qui occasionnent en nous des sensations, et qui ont des propriétés sur lesquelles nous ne saurions rien assurer. 
Plus de certitude à cet égard nous serait inutile.

La statue ne s'arrêtera vraisemblablement pas à ces doutes. Peutêtre les jugements, dont elle s'est fait une habitude, ne lui permettrontils pas de les former. Elle en serait cependant plus capable que nous, parce qu'elle sait mieux comment elle a appris à voir, à entendre, à sentir, à goûter, à toucher. Quoi qu'il en soit, il lui est inutile d'avoir plus de certitude à cet égard. L'apparence des qualités sensibles suffit pour lui donner des désirs, pour éclairer sa conduite, et pour faire son bonheur ou son malheur; et la dépendance où elle est des objets auxquels elle est obligée de les rapporter, ne lui permet pas de douter qu'il existe des êtres hors d'elle. Mais quelle est la nature de ces êtres ? Elle l'ignore, et nous l'ignorons nous-mêmes. Tout ce que nous savons, c'est que nous les appelons corps.

Table des matières

Mais, insistera-t-on, il est décidé par l'écriture que les corps sont étendus, et vous rendez au moins la chose douteuse.

Si cela est, la foi rend certain ce qui est douteux en philosophie, et il n'y a point là de contradiction. En pareil cas le philosophe doit douter, quand il consulte sa raison ; comme il doit croire, quand la révélation l'éclaire. Mais l'écriture ne décide rien à ce sujet. Elle suppose les corps étendus, comme elle les suppose colorés, sonores, etc., et certainement c'est là une de ces questions que Dieu a voulu abandonner aux disputes des philosophes. 


\section{Chapitre VI \\ Considérations sur les idées abstraites et générales, que peut acquérir un homme qui vit hors de toute société}

L'histoire que nous venons de faire des connaissances de notre statue, montre sensiblement comment elle distribue les êtres en différentes classes, suivant leurs rapports à ses besoins ; et, par conséquent, comment elle se fait des notions abstraites et générales. Mais pour mieux connaître la nature de ses idées, il est important d'entrer dans de nouveaux détails.

La statue n’a point d'idée générale, qui n’ait été particulière.

Elle n'a point d'idée générale, qui n'ait d'abord été particulière. L'idée générale d'orange, par exemple, n'est dans son origine que l'idée de telle orange.

En quoi consiste l'idée qu'elle a d'un objet présent.

L’idée particulière, lorsqu'un objet est présent aux sens, c'est la collection de plusieurs qualités qui se montrent ensemble. L'idée de telle orange c'est la couleur, la forme, la saveur, l'odeur, la solidité, le poids, etc.

\section{D'un objet absent.}

Cette idée particulière, quand l'objet n'agit plus sur les sens, c'est le souvenir qui reste de ce qu'on a connu à la vue, au goût, à l'odorat, etc. Fermez les yeux ; l'idée de la lumière est le souvenir d'une impression que vous avez éprouvée : ne touchez rien ; l'idée de solidité 
est le souvenir de la résistance que vous avez rencontrée, en maniant des corps : ainsi du reste.

\section{Comment, de particulières, ses idées deviennent générales.}

Substituons successivement, une à une, plusieurs oranges à la première, et qu'elles soient toutes semblables ; notre statue croira toujours voir la même, et elle n'aura à ce sujet qu'une idée particulière.

En voit-elle deux à-la-fois ? Aussitôt elle reconnaît dans chacune la même idée particulière, et cette idée devient un modèle, auquel elle les compare, et avec lequel elle voit qu'elles conviennent l'une et l'autre. Elle découvrira de la même manière que cette idée est commune à trois, quatre, cinq, six oranges, et elle la rendra aussi générale qu'elle peut l'être pour elle.

L'idée particulière d'un cheval et celle d'un oiseau deviendront également générales, lorsque les circonstances feront comparer plusieurs chevaux et plusieurs oiseaux ; et ainsi de tous les objets sensibles.

Comme la statue n'a l'usage d'aucun signe, elle ne peut pas classer ses idées avec ordre, ni par conséquent, en avoir d'aussi générales que nous. Mais elle ne peut pas non plus n'avoir absolument point d'idées générales. Si un enfant qui ne parle pas encore, n'en avait pas d'assez générales pour être communes au moins à deux ou trois individus, on ne pourrait jamais lui apprendre à parler, car on ne peut commencer à parler une langue, que parce qu'avant de la parler, on a quelque chose à dire, que parce qu'on a des idées générales : toute proposition en renferme nécessairement.

Ayant les notions générales d'orange, de cheval, d'oiseau; notre statue les distinguera, par la même raison, qu'elle distingue une orange d'un oiseau, un oiseau d'un cheval. Elle rapportera donc chacun de ces individus au modèle général dont elle s'est fait l'idée, c'est-à-dire, à la classe, à l'espèce à laquelle il appartient.

Or, comme un modèle qui convient à plusieurs individus, est une idée générale ; de même deux, trois modèles, sous lesquels on arrange des individus tous différents, sont différentes classes, ou pour parler le langage des philosophes, différentes espèces de notions générales. 
Comment d'une idée générale, elle descend à de moins générales.

Lorsqu'elle jette les yeux sur une campagne, elle aperçoit quantité d'arbres, dont elle ne remarque point encore la différence ; elle voit seulement ce qu'ils ont de commun : elle voit qu'ils portent chacun des branches, des feuilles, et qu'ils sont arrêtés à l'endroit où ils croissent. Voilà le modèle de l’idée générale d'arbre.

Elle va ensuite des uns aux autres : elle observe la différence des fruits, elle se fait des modèles, par où elle distingue autant de sortes d'arbres, qu'elle remarque d'espèces de fruits ; et ce sont là des idées moins générales que la première.

Elle se fera de même l'idée générale d'animal, si elle voit dans l'éloignement plusieurs animaux, dont la différence lui échappe ; et elle les distinguera en plusieurs espèces, lorsqu'elle sera à portée de voir en quoi ils différent.

Elle généralise, à proportion qu'elle voit plus confusément.

Elle généralise donc davantage à proportion qu'elle voit d'une manière plus confuse ; et elle se fait des notions moins générales, à proportion qu'elle démêle plus de différence dans les choses. On voit par là combien il lui est facile de se faire des idées générales ${ }^{38}$.

D'abord toutes les pommes, par exemple, lui paraissent conformes au même modèle. Mais dans la suite, elle ne trouve pas à chacune une saveur également agréable. Dès lors, le désir du plaisir et la crainte du dégoût les lui font comparer, sous les rapports qu'elle y peut découvrir : elle apprend à les distinguer à la vue, à l'odorat, au toucher ; elle s'en forme différents modèles propres à éclairer son choix ; et elle les distribue en autant de classes, qu'elle y remarque de différences.

38 La distribution des êtres en différentes espèces, n’a donc pour principe que l'imperfection de notre manière de voir. Elle n'est donc pas fondée dans la nature des choses, et les philosophes ont eu tort de vouloir déterminer l'essence de chaque espèce d'être. Voilà cependant ce qui a été de tout temps l'objet de leurs recherches. Cette erreur vient de ce qu'ils étaient persuadés que nos idées avaient été gravées en nous par la main d'un Dieu, qui, avant de nous les donner, avait sans doute consulté la nature des choses. 
Objets dont elle ne prend aucune connaissan-

ce.

Quant aux objets qui ne l'intéressent ni par le plaisir, ni par la peine, ils restent confondus dans la foule, et elle n'en acquiert aucune connaissance.

Il ne faut que réfléchir sur nous, pour se convaincre de cette vérité. Tous les hommes ont les mêmes sensations ; mais le peuple occupé à des travaux pénibles, l'homme du monde tout entier à des objets frivoles, et le philosophe, qui s'est fait un besoin de l'étude de la nature, ne sont sensibles ni aux mêmes plaisirs, ni aux mêmes peines. Aussi tirent-ils des mêmes sensations des connaissances bien différentes.

Dans quel ordre elle se fait des idées d'espèce.

Voici donc l'ordre dans lequel notre statue se fait des idées d'espèce. D'abord elle n'aperçoit que les différences les plus sensibles, et elle a des idées très générales ; mais en petit nombre.

Si c'est la couleur qui la frappe davantage, elle ne fera qu'une classe de plusieurs espèces de fleurs : si c'est le volume, un levreau et un chat ne seront pour elle qu'une seule espèce d'animal.

Les besoins lui donnent ensuite occasion de considérer les objets par d'autres qualités, elle fera des espèces subordonnées aux premières. D'une notion générale, il s'en formera plusieurs qui le seront moins.

Elle passe donc tout d'un coup des idées particulières aux plus générales ; d'où elle descend à de moins générales, à mesure qu'elle remarque la différence des choses. C'est ainsi qu'un enfant, après avoir appelé or, tout ce qui est jaune, acquiert ensuite les idées de cuivre, de tombac ; et d'une idée générale en fait plusieurs qui le sont moins.

Son ignorance sur la nature des choses.

Par la génération de ces idées, il est évident qu’elles ne présenteront à notre statue que des qualités différemment combinées. Elle voit, par exemple, la solidité, l'étendue, la divisibilité, la figure, la mobilité, etc. Réunies dans tout ce qu'elle touche ; et elle a, par conséquent, l'idée de corps. Mais si on lui demandait ce que c'est qu'un corps, et 
qu'elle pût répondre ; elle en montrerait un, et dirait, c'est cela : c'està-dire, cela où vous trouvez tout-à-la-fois de la solidité, de l'étendue, de la divisibilité, de la figure, etc.

\section{Commune aux philosophes.}

Un philosophe répondrait ; c'est un être, une substance étendue, solide, etc. Comparons ces deux réponses, et nous verrons qu'il ne connaît pas mieux qu'elle la nature du corps. Son seul avantage, si c'en est un, c'est de s'être fait un langage, qui ne paraît savant, que parce qu'il n'est pas celui de tout le monde. Car dans le vrai, les mots être, substance, ne signifient rien de plus, que le mot cela.

Les idées qu'elle a des objets, sont confuses.

De là, il faut conclure que les idées qu'elle a des objets sensibles, sont confuses ; car j'appelle confuse, toute idée qui ne représente pas d'une manière distincte toutes les qualités de son objet. Or, il n'est point de corps, dont elle ait une connaissance aussi parfaite ; elle n'y voit que les propriétés, que ses besoins lui donnent occasion d'y remarquer. Avec plus de sagacité elle en démêlerait un plus grand nombre, et si elle pouvait pénétrer jusque dans la nature des êtres, elle n’en trouverait pas deux parfaitement semblables. Elle ne suppose donc que plusieurs ne différent point entre eux, que parce qu'elle les voit confusément.

Ses idées abstraites sont de deux espèces.

Quant à ses notions abstraites, il y en a de confuses et de distinctes. 
Les unes confuses.

Elle connaît par exemple assez bien un son, pour le distinguer d'une odeur, d'une saveur, et de tout autre son ; mais il lui paraît simple, quoique multiple ${ }^{39}$. Plusieurs couleurs, mêlées ensemble, ne produisent à son égard que l'apparence d'une seule. Il en est de même de toutes les impressions des sens. Elle ne démêle donc pas tout ce qu'elles renferment ; et elle est encore plus éloignée de découvrir toutes les causes qui concourent à chaque sensation. Elle n'a donc à ce sujet, que des notions fort confuses.

Les autres distinctes.

Mais ces mêmes sensations lui donnent des idées de grandeur et de figure ; et si elle ne peut assurer qu'elle est précisément la grandeur et la figure des corps, ni déterminer exactement les rapports qu'ils ont entre eux ; elle sait comment une grandeur peut être le double, ou la moitié d'une autre, et elle connaît fort bien une ligne, un triangle, un carré. Elle a donc, en pareil cas, des idées distinctes. Il suffit pour cela qu'elle considère les grandeurs, en faisant abstraction des objets.

\section{Elle connaît deux sortes de vérités.}

De ces deux sortes d'idées naissent deux sortes de vérités. Lorsque la statue remarque qu'un corps est triangulaire, elle porte un jugement qui peut devenir faux ; car ce corps peut changer de figure. Mais lorsqu'elle remarque qu'un triangle a trois côtés, son jugement est vrai, et le sera toujours; puisque trois côtés déterminent l’idée du triangle. Elle aperçoit donc des vérités qui changent, ou qui peuvent changer toutes les fois qu'elle veut juger de ce que les choses sont en ellesmêmes ; elle aperçoit au contraire des vérités qui ne changent point, toutes les fois qu'elle se borne à juger des idées distinctes et abstraites, qu'elle a des grandeurs.

Elle a, par conséquent, avec le seul secours des sens, des connaissances de toute espèce.

Table des matières

39 Cela est évident du bruit, et n'est pas moins certain des sons harmoniques ; car on a remarqué qu'il n’en est point qui ne soit triple. 


\section{Chapitre VII}

\section{D’un homme trouvé dans les forêts de Lithuanie}

\section{Circonstances où le besoin de nourriture engourdit toutes les facultés de l’âme.}

Notre statue, comme nous l'avons remarqué, pourrait être si fort occupée du soin de sa nourriture, qu'elle n'aurait pas un moment à donner à l'étude des objets dont elle était curieuse avant qu'elle eût l'organe du goût. Ne vivant que pour satisfaire à ce pressant besoin, les plaisirs des autres sens n'auraient plus d'attrait pour elle : elle ne remarquerait plus les objets qui pourraient les produire. Sans étonnement, sans curiosité, elle cesserait de réfléchir sur ce qu'elle a su, elle en oublierait bientôt une partie, elle oublierait comment elle a appris ce qu'elle sait encore ; et elle ne douterait pas qu'elle n'eût toujours senti, entendu, vu et touché comme elle sent, entend, voit et touche. Toute entière à la recherche d'une nourriture, que je suppose extrêmement rare, elle mènerait une vie purement animale. A-t-elle faim ? Elle se meut, elle va partout où elle se souvient d'avoir trouvé des aliments. Sa faim est-elle dissipée, le repos devient son besoin le plus pressant ; elle reste où elle est, elle s'endort.

Dans de pareilles circonstances, le besoin de nourriture engourdit donc à certains égards les facultés de son âme : il tourne vers lui toute leur action. Il est même vraisemblable, qu'au lieu de se conduire d'après sa propre réflexion, elle prendrait des leçons des animaux, avec qui elle vivrait plus familièrement. Elle marcherait comme eux, imiterait leurs cris, brouterait l'herbe, ou dévorerait ceux dont elle aurait la force de se saisir. Nous sommes si fort portés à l'imitation, qu'un Descartes à sa place n'apprendrait pas à marcher sur ses pieds : tout ce qu'il verrait, suffirait pour l'en détourner. 
Enfant trouvé dans les forêts de Lithuanie.

Tel était vraisemblablement le sort d'un enfant d'environ dix ans, qui vivait parmi les ours, et qu'on trouva en 1694, dans les forêts qui confinent la Lithuanie et la Russie. Il ne donnait aucune marque de raison, marchait sur ses pieds et sur ses mains, n'avait aucun langage, et formait des sons qui ne ressemblaient en rien à ceux d'un homme. Il fut longtemps avant de pouvoir proférer quelques paroles, encore le fit-il d'une manière bien barbare. Aussitôt qu'il put parler, on l'interrogea sur son premier état ; mais il ne s'en souvint non plus que nous nous souvenons de ce qui nous est arrivé au berceau.

\section{Pourquoi on dit qu'il ne donnait aucun signe \\ de raison.}

Quand on dit que cet enfant ne donnait aucun signe de raison, ce n'est pas qu'il ne raisonnât suffisamment pour veiller à sa conservation ; mais c'est que sa réflexion, jusqu'alors appliquée nécessairement à ce seul objet, n'avait point eu occasion de se porter sur ceux dont nous nous occupons. Il n'avait aucune des idées que notre statue a acquises, lorsqu'elle connaissait d'autres besoins que celui de chercher des aliments : il manquait de toutes les connaissances que les hommes doivent à leur commerce réciproque. En un mot, il paraissait sans raison, non qu'absolument il n'en eût point ; mais parce qu'il en avait moins que nous.

\section{Pourquoi il oublia son premier état.}

Quelquefois notre conscience partagée entre un grand nombre de perceptions, qui agissent sur nous avec une force à-peu-près égale, est si faible, qu'il ne nous reste aucun souvenir de ce que nous avons éprouvé. A peine sentons-nous pour lors que nous existons : des jours s'écouleraient comme des moments, sans que nous en fissions la différence ; et nous éprouverions des milliers de fois la même perception, sans remarquer que nous l'avons déjà eue. Un homme qui a acquis beaucoup d'idées, et qui se les est rendues familières, ne peut pas demeurer longtemps dans cette espèce de léthargie. Plus la provision de ses idées est grande, plus il y a lieu de croire que quelqu'une aura occasion de se réveiller, d'exercer son attention d'une manière particulière, et de le retirer de cet assoupissement. Cet enfant n'avait pas un pareil secours. Ses facultés engourdies ne pouvaient être secouées, 
que par le besoin de chercher de la nourriture ; et sa vie ressemblait à un sommeil, qui ne serait interrompu que par des songes. Il était donc naturel qu'il oubliât son premier état.

Cependant, il n'est pas vraisemblable qu'il en perdît tout-à-coup le souvenir. Si au bout de quelques jours, on l'eût ramené dans les bois où on l'avait pris, il eût sans doute reconnu les lieux où il avait vécu ; il se fût rappelé les aliments dont il s'était nourri ; et les moyens qu'il avait employés pour se les procurer: il n'eût pas eu besoin de s'instruire une seconde fois de toutes ces choses ; mais le souvenir en fut effacé par de nouvelles idées, et surtout par le long intervalle qui s'écoula jusqu'au moment où il fut en état de répondre aux questions qu'on lui fit. Néanmoins, pour mieux s'en assurer, il eût fallu le reconduire dans les forêts où il avait été trouvé. Quoiqu'il ne se souvînt pas de ces lieux, quand on lui en parlait, peut-être aurait-il su les reconnaître quant il les aurait vus. 
Table des matières

\section{Chapitre VIII}

\section{D'un homme qui se souviendrait d'avoir reçu successivement l'usage de ses sens}

En supposant que notre statue se souvînt de l'ordre dans lequel les sens lui ont été accordés ; il suffirait de la faire réfléchir sur ellemême, pour remettre sous les yeux les principales vérités que nous avons démontrées.

La statue compare l'état où elle est à celui où

elle était, quand elle ne connaissait rien hors

d'elle.

Que suis-je, dirait-elle, et qu'ai-je été ? Qu'est-ce que ces sons, ces odeurs, ces saveurs, ces couleurs, que $\mathrm{j}$ 'ai pris successivement pour mes manières d'être, et que les objets paraissent aujourd'hui m'enlever ? Qu'est-ce que cette étendue, que je découvre en moi et au-delà, sans bornes ? Ne serait-ce que différentes manières de me sentir ? Avant que la vue me fût rendue, l'espace des cieux m'était inconnu : avant que j'eusse l'usage de mes membres, j'ignorais qu'il y eût quelque chose hors de moi. Que dis-je ? Je ne savais pas que je fusse étendue : je n'étais qu'un point, lorsque j'étais réduite au sentiment uniforme. Quelle est donc cette suite de sentiments, qui m'a fait ce que je suis ; et qui peut-être a fait ce qu'est à mon égard tout ce qui m'environne?

Je ne sens que moi, et c'est dans ce que je sens en moi, que je vois au-dehors : ou plutôt je ne vois pas au-dehors ; mais je me suis fait une habitude de certains jugements, qui transportent mes sensations où elles ne sont pas. 
Au premier moment de mon existence, je ne savais point ce qui se passait en moi ; je n’y démêlais rien encore ; je n'avais aucune conscience de moi-même ; j'étais, mais sans désirs, sans crainte, je jouissais à peine de moi : et, si j'eusse continué d'exister de la sorte, je n'aurais jamais soupçonné que mon existence pût embrasser deux instants.

Mais j'éprouve successivement plusieurs sensations: elles occupent ma capacité de sentir, à proportion des degrés de peine ou de plaisir, qui les accompagnent. Par là elles restent présentes à ma mémoire, lorsqu'elles ne le sont plus à mon organe. Mon attention étant partagée entre elles, je les compare, je juge de leurs rapports, je m'en fais des idées abstraites, je connais des vérités générales.

Alors, toute l'activité dont je suis capable, se porte aux manières d'être qui m'ont plu davantage ; j'ai des besoins, je forme des désirs, j'aime, je hais, j'espère, je crains, j'ai des passions ; et ma mémoire m’obéit quelquefois avec tant de vivacité, que je m'imagine éprouver des sensations, que je ne fais que me rappeler.

Étonnée de ce qui se passe en moi, je m’observe avec encore plus d'attention, à chaque instant je sens que je ne suis plus ce que j'ai été. Il me semble que je cesse d'être moi, pour redevenir un autre moimême. Jouir et souffrir font tour-à-tour mon existence ; et par la succession de mes manières d'être, je m'aperçois que je dure. Il fallait donc que ce moi variât à chaque instant, au hasard de se changer souvent contre un autre, où il m'est douloureux de me retrouver.

Plus je compare mes manières d'être, plus la jouissance ou la souffrance m'en est sensible. Le plaisir et la douleur continuent à l'envi d'attirer mon attention: l'un et l'autre développent toutes mes facultés : je ne me fais des habitudes, que parce que je leur obéis ; et je ne vis plus que pour désirer ou pour craindre.

Elle se rappelle comment elle a découvert son corps, et d'autres objets.

Mais bientôt je suis à-la-fois de plusieurs manières. Accoutumée à les remarquer, lorsqu'elles se succèdent, je les remarque encore, lorsque je les éprouve ensemble ; et mon existence me paraît se multiplier dans un même moment. 
Cependant je porte les mains sur moi-même, je les porte sur ce qui m'environne. Aussitôt une nouvelle sensation semble donner du corps à toutes mes manières d'être. Tout prend de la solidité sous mes mains. Étonnée de ce nouveau sentiment, je le suis encore plus de ne me pas retrouver dans tout ce que je touche. Je me cherche où je ne suis pas : il me semble que j'avais seule le droit d'exister ; et que tout ce que je rencontre, se formant aux dépens de mon être, ne se fait connaître à moi que pour me réduire à des limites toujours plus étroites. Que deviens-je en effet, lorsque je compare le point où je suis, avec l'espace que remplit cette multitude d'objets que je découvre?

Dès ce moment, il me semble que mes manières d'être cessent de m’appartenir : j'en fais des collections hors de moi : j'en forme tous les objets, dont je prends connaissance. Des idées qui demandent moins de comparaisons, je m’élève aux idées, que je n’acquiers qu'autant que je combine. Je conduis mon attention d'un objet à un autre, et rassemblant dans la notion que je me forme de chacun, les idées et les rapports que j’y remarque, je réfléchis sur eux.

Si je me suis d'abord mue par le seul plaisir de me mouvoir, je me meus bientôt dans l'espérance de rencontrer de nouveaux plaisirs ; et devenant capable de curiosité, je passe continuellement de la crainte à l'espérance, du mouvement au repos : quelquefois j'oublie ce que j'ai souffert, d'autres fois je me précautionne contre les maux dont je suis menacée : enfin le plaisir et la douleur, seuls principes de mes désirs, m'apprennent à me conduire dans l'espace, et à me faire à toute occasion de nouvelles idées.

\section{Elle se rappelle comment le toucher instruit} les autres sens.

Pourrais-je avoir d'autres facultés que celles de me mouvoir et de manier des corps ? Je ne l'imaginais pas ; car j'avais totalement perdu le souvenir de ce que j'ai été. Quelle fut donc ma surprise, lorsque je me retrouvai son, saveur, odeur, lumière et couleur ! Bientôt il me semble que je me suis laissé séduire à une illusion, que le toucher paraît dissiper. Je juge que toutes ces manières d'être me viennent des corps ; et je me fais une si grande habitude de les sentir, comme si elles y étaient en effet, que j'ai peine à croire qu'elles ne leur appartiennent pas. 
Quoi de plus simple que la manière dont j'ai appris à me servir de mes sens!

J'ouvre les yeux à la lumière, et je ne vois d'abord qu'un nuage confus. Je touche, j'avance, je touche encore : un chaos se débrouille insensiblement à mes regards. Le tact décompose en quelque sorte la lumière ; il sépare les couleurs, les distribue sur les objets, démêle un espace éclairé, et dans cet espace des grandeurs et des figures, conduit mes yeux jusqu'à une certaine distance, leur ouvre le chemin par où ils doivent se porter au loin sur la terre, et s'élever jusqu'aux cieux : devant eux, en un mot, il déploie l'univers. Alors ils paraissent se jouer dans des espaces immenses ; ils manient les objets, auxquels le toucher ne peut atteindre ; ils les mesurent ; et les parcourant avec une rapidité étonnante, ils semblent enlever ou donner à mon gré l'existence à toute la nature. Au seul mouvement de ma paupière, je crée ou j’anéantis tout ce qui m’environne.

Quand je ne jouissais pas de ce sens, aurais-je jamais pu comprendre comment, ne changeant point de place, il m'aurait été possible de connaître ce qui est hors de la portée de ma main ? Quelle idée me serais-je fait d'un organe qui saisit à une si grande distance les formes et les grandeurs ? Est-ce un bras qui s'allonge d'une manière extraordinaire pour aller jusqu'à elles, ou viennent-elles jusqu'à lui ? Pourquoi se porte-t-il au-delà de certains corps, tandis qu'il est arrêté par d'autres ? Comment touche-t-il dans les eaux les mêmes objets, qu'il touche encore au-dehors ? Est-ce une illusion, ou en effet toute la nature se reproduit-elle?

Il me semble qu'à chaque objet que j'étudie, je me fais une nouvelle manière de voir, et me procure un nouveau plaisir. Ici c'est une plaine vaste, uniforme, où ma vue, passant par-dessus tout ce qui est près de moi, se porte à une distance indéterminée ; et se perd dans un espace qui m’étonne. Là, c'est un pays coupé et plus borné, où mes yeux après s'être reposés sur chaque objet, embrassent un tableau plus distinct et plus varié. Des tapis de verdure, des bosquets de fleurs, des massifs de bois, où le soleil pénètre à peine ; des eaux qui coulent lentement ou qui se précipitent avec violence, embellissent ce paysage, que paraît animer une lumière qui répand sur lui mille couleurs différentes. Immobile à cette vue, tout appelle mes regards. A peine je les détourne, que je ne sais si je les dois fixer sur les objets que je viens 
de découvrir, ou les reporter sur ceux que je viens de perdre. Je les conduis avec inquiétude des uns aux autres ; et mieux je démêle toutes les sensations dont je jouis, plus je suis sensible au plaisir de voir.

Curieuse, je parcours avec empressement des lieux dont le premier aspect m'a ravie ; et j'aime à reconnaître à l'ouïe, à l'odorat, au goût et au toucher, les objets qui me frappent les yeux de toute part. Toutes mes sensations semblent craindre de céder les unes aux autres. La variété et la vivacité des couleurs le disputent au parfum des fleurs ; les oiseaux me paraissent plus admirables par leur forme, leur mouvement et leur plumage, que par leurs chants. Et qu'est-ce que le murmure des eaux comparé à leur cours, leurs cascades et leur brillant cristal !

Tel est le sens de la vue : à peine instruit par le toucher, il dispense les trésors dans la nature; il les prodigue pour décorer les lieux que son guide lui découvre; et il fait des cieux et de la terre un spectacle enchanteur, qui n'a de magnificence, que parce qu'il y répand ses propres sensations.

Elle se rappelle comment les plaisirs et les peines ont été le premier mobile de ses facultés.

Que serais-je donc, si toujours concentrée en moi-même, je n’avais jamais su transporter mes manières d'être hors de moi ? Mais dès que le toucher instruit mes autres sens, je vois au-dehors des objets qui attirent mon attention par les plaisirs ou par les peines qu'ils me causent. Je les compare, j'en juge, je sens le besoin de les rechercher, ou de les fuir ; je les désire, je les aime, je les hais, je les crains : chaque jour j'acquiers de nouvelles connaissances; et tout ce qui m'environne devient l'instrument de ma mémoire, de mon imagination et de toutes les opérations de mon âme.

Pourquoi faut-il que je trouve des obstacles à mes désirs ? Pourquoi faut-il que mon bonheur soit traversé par des peines ? Mais que dis-je ! Jouirais-je proprement des biens qui me sont offerts, si je n'avais jamais de victoire à remporter? En jouirais-je si les maux, dont je me plains, ne m'en faisaient pas connaître le prix ? Mon malheur même contribue à mon bonheur ; et la plus grande jouissance des biens naît de l'idée vive des maux auxquels je les compare. C’est au 
retour des uns et des autres, que je dois toutes mes connaissances, que je dois tout ce que je suis.

De là, mes besoins, mes désirs, et les différents intérêts qui sont le mobile de mes actions ; en sorte que je n'étudie les choses qu'à proportion que j'y crois découvrir des plaisirs à rechercher, ou des peines à fuir. Voilà la lumière qui éclaire les objets suivant les rapports qu'ils ont à moi : elle répand sur eux différents jours pour me les faire distribuer en différentes classes; et ceux qui sont soustraits à ses rayons sont ensevelis dans des ténèbres où je ne puis les découvrir.

J'étudie les fruits, et tout ce qui est propre à me nourrir ; je cherche les moyens de m'en procurer la jouissance: j'étudie les animaux, j'observe ceux qui peuvent me nuire, j'apprends à me garantir de leurs coups : enfin j'étudie tout ce qui flatte ma curiosité : je me fais, selon mes passions, des règles pour juger de la bonté et de la beauté des choses. Tantôt je prends des précautions que je crois nécessaires à mon bonheur ; tantôt j'invite les objets à y travailler eux-mêmes : et il me semble que je ne suis entourée que d'êtres amis ou ennemis.

Instruite par l'expérience, j'examine, je délibère avant d'agir. Je n’obéis plus aveuglément à mes passions, je leur résiste, je me conduis d'après mes lumières, je suis libre ; et je fais un meilleur usage de ma liberté, à proportion que j’ai acquis plus de connaissances.

Elle réfléchit sur les jugements dont elle s'est

fait une habitude.

Mais quelle est la certitude de ces connaissances ? Je ne vois proprement que moi, je ne jouis que de moi : car je ne vois que mes manières d'être, elles sont ma seule jouissance; et si mes jugements d'habitude me donnent tant de penchant à croire qu'il existe des qualités sensibles au-dehors, ils ne me le démontrent pas. Je pourrais donc être telle que je suis, avoir les mêmes besoins, les mêmes désirs, les mêmes passions, quand même les objets que je recherche ou que j'évite, n'auraient aucune de ces qualités. En effet, sans le toucher, j'aurais toujours regardé les odeurs, les saveurs, les couleurs et les sons comme à moi ; jamais je n'aurais jugé qu'il y a des corps odoriférants, sonores, colorés, savoureux. Comment donc pourrais-je être assurée de ne me pas tromper, lorsque je juge qu'il y a de l'étendue ? 
Mais il m'importe peu de savoir avec certitude, si ces choses existent ou n'existent pas. J'ai des sensations agréables ou désagréables : elles m'affectent autant que si elles exprimaient les qualités mêmes des objets auxquels je suis portée à les attribuer ; et c'en est assez pour veiller à ma conservation. A la vérité les idées que je me forme des choses sensibles, sont confuses; je n'en marque les rapports qu'imparfaitement. Mais je n'ai qu'à faire quelques abstractions, pour avoir des idées distinctes, et pour apercevoir des rapports plus exacts. Aussitôt je remarque deux sortes de vérités : les unes peuvent cesser d’être ; les autres ont été, sont et seront toujours.

Elle réfléchit sur l'ignorance où elle est d'elle-

même.

Cependant, si je connais imparfaitement les objets extérieurs, je ne me connais pas mieux moi-même. Je me vois formée d'organes propres à recevoir différentes impressions; je me vois environnée d'objets qui agissent tous sur moi, chacun à sa manière ; enfin dans le plaisir et dans la peine qui accompagnent constamment les sensations que j'éprouve, je crois apercevoir le principe de ma vie et de toutes mes facultés.

Mais ce moi qui prend de la couleur à mes yeux, de la solidité sous mes mains, se connaît-il mieux pour regarder aujourd'hui comme à lui toutes les parties de ce corps auxquelles il s'intéresse, et dans lesquelles il croit exister ? Je sais qu'elles sont à moi, sans pouvoir le comprendre : je me vois, je me touche, en un mot, je me sens, mais je ne sais ce que je suis ; et si j'ai cru être son, saveur, couleur, odeur, actuellement je ne sais plus ce que je dois me croire. 


\section{Chapitre IX}

\section{Conclusion}

\section{Dans l'ordre naturel, tout vient des sensations.}

Nous ne saurions nous appliquer toutes les suppositions que j'ai faites : mais elles prouvent au moins que toutes nos connaissances viennent des sens, et particulièrement du toucher ; parce que c'est lui qui instruit les autres. Si en ne supposant que des sensations dans notre statue, elle a acquis des idées particulières et générales, et s'est rendue capable de toutes les opérations de l'entendement; si elle a formé des désirs, et s'est fait des passions auxquelles elle obéit ou résiste ; enfin si le plaisir et la douleur sont l'unique principe du développement de ses facultés, il est raisonnable de conclure que nous n'avons d'abord eu que des sensations, et que nos connaissances et nos passions sont l'effet des plaisirs et des peines qui accompagnent les impressions des sens.

En effet, plus on y réfléchira, plus on se convaincra que c'est là l'unique source de notre lumière et de nos sentiments. Suivons la lumière : aussitôt nous jouissons d'une vie nouvelle, et bien différente de celle que procuraient auparavant des sensations brutes, si j'ose m'exprimer ainsi. Suivons le sentiment, observons-le surtout lorsqu'il s'accroît de tous les jugements que nous nous sommes accoutumés à confondre avec les impressions des sens : aussitôt de ces sensations, qui ne présentaient d'abord qu'un petit nombre de plaisirs grossiers, vont naître des plaisirs délicats, qui se succéderont dans une variété étonnante. Ainsi plus nous nous éloignerons de ce que les sensations étaient au commencement, plus la vie de notre être se développera, se variera : elle s'étendra à tant de choses, que nous aurons de la peine à comprendre, comment toutes nos facultés peuvent avoir un principe commun dans la sensation. 
Cette source n'est pas également abondante pour tous les hommes.

Tant que les hommes ne remarquent encore dans les impressions des sens que des sensations où ils n'ont su mêler que peu de jugements, la vie de l'un est à-peu-près semblable à celle de l'autre : il n'y a presque de différence que dans le degré de vivacité, avec lequel ils sentent. L'expérience et la réflexion seront pour eux, ce qu'est le ciseau entre les mains du sculpteur, qui découvre une statue parfaite dans une pierre informe ; et suivant l'art avec lequel ils manieront ce ciseau, ils verront sortir de leurs sensations une nouvelle lumière et de nouveaux plaisirs.

Si nous les observons, nous connaîtrons comment ces matériaux restent grossiers ou sont mis en œuvre ; et, considérant l'intervalle que les hommes laissent entre eux, nous serons étonnés combien, dans un même espace de temps, les uns vivent plus que les autres : car vivre, c'est proprement jouir, et la vie est plus longue pour qui sait davantage multiplier les objets de sa jouissance.

Nous avons vu que la jouissance peut commencer à la première sensation agréable. Au premier moment, par exemple, que nous accordons la vue à notre statue, elle jouit ; ses yeux ne fussent-ils frappés que d'une couleur noire. Car il ne faut pas juger de ses plaisirs par les nôtres. Plusieurs sensations nous sont indifférentes, ou même désagréables, soit parce qu'elles n'ont rien de nouveau pour nous, soit parce que nous en connaissons de plus vives. Mais sa situation est bien différente ; et elle peut être transportée, lorsqu'elle éprouve des sentiments que nous ne daignons pas remarquer, ou que nous ne remarquons qu'avec dégoût.

Observons la lumière, quand le toucher apprend à l'œil à répandre les couleurs dans toute la nature : voilà autant de nouveaux sentiments, et par conséquent autant de nouveaux plaisirs, autant de nouvelles jouissances.

Il faut raisonner de même sur tous les autres sens et sur toutes les opérations de l'âme. Car nous jouissons non seulement par la vue, l'ouïe, le goût, l'odorat, le toucher ; nous jouissons encore par la mémoire, l'imagination, la réflexion, les passions, l'espérance, en un 
mot, par toutes nos facultés. Mais ces principes n’ont pas la même activité chez tous les hommes.

\section{L'homme n'est rien, qu'autant qu'il a acquis.}

Ce sont les plaisirs et les peines comparés, c’est-à-dire, nos besoins qui exercent nos facultés. Par conséquent, c'est à eux que nous devons le bonheur que nous avons à jouir. Autant de besoins, autant de jouissances différentes ; autant de degrés dans le besoin, autant de degrés dans la jouissance. Voilà le germe de tout ce que nous sommes, la source de notre malheur ou de notre bonheur. Observer l'influence de ce principe, c'est donc le seul moyen de nous étudier nous-mêmes.

L'histoire des facultés de notre statue rend sensible le progrès de toutes ces choses. Lorsqu'elle était bornée au sentiment fondamental, une sensation uniforme était tout son être, toute sa connaissance, tout son plaisir. En lui donnant successivement de nouvelles manières d'être et de nouveaux sens, nous l'avons vue former des désirs, apprendre de l'expérience à les régler ou à les satisfaire, et passer de besoins en besoins, de connaissances en connaissances, de plaisirs en plaisirs. Elle n'est donc rien qu'autant qu'elle a acquis. Pourquoi n'en serait-il pas de même de l'homme? 


\section{DISSERTATION SUR LA LIBERTÉ}

Table des matières

\section{Avant-propos}

Des observations sur un homme qui n'a encore contracté aucune sorte d'habitude, doivent être regardées comme les commencements de l'histoire de l'esprit humain : il me paraît qu'elles détruisent dans le principe tous les systèmes métaphysiques, qui sont nés des préjugés, et qu'elles dispensent de jeter les yeux sur cette multitude d'opinions qui voilent la vérité, l'altèrent ou la combattent. C'est pour en donner un exemple sensible que je joins ici une Dissertation sur la Liberté. Comme il n'y a peut-être pas de question sur laquelle on ait plus écrit, ni avec plus de subtilité, elle sera très propre à montrer les avantages de la méthode que nous avons suivie dans le Traité des Sensations.

Dissertation sur la liberté

Suppositions où la statue ne trouve point

d'obstacles à ses désirs.

Supposons que notre statue ne trouve jamais d'obstacle à ses désirs, qu'elle ne soit jamais exposée à aucune peine pour les avoir satisfaits, et qu'elle jouisse toujours de ce qui peut lui faire le plus grand plaisir ; en ce cas, elle ne connaîtra pas la crainte, elle vivra sans précaution, et obéira sans inquiétude à tous ses penchants. 
Où ses désirs sont en équilibre.

A-t-elle tout-à-la-fois plusieurs besoins également pressants ? Elle a plusieurs désirs qui agissent avec des forces égales : aucun ne peut vaincre ; elle flotte entre plusieurs objets, et elle ne se porte pas plus à l'un qu'à l'autre.

Où ils sont supérieurs les uns aux autres.

Mais, s'il survient une circonstance qui lui retrace plus vivement le plaisir de jouir d'un de ces objets, l'inquiétude que produit la privation de ce plaisir en devient plus grande. De là naît un désir qui trouve dans les autres d'autant moins de résistance, qu'il leur est plus supérieur, et qui les soumet quelquefois si rapidement, qu'il ne paraît presque pas avoir eu à les combattre.

Varions souvent les circonstances, à chaque changement ce sera un nouveau besoin qui dominera, et la statue ira de désirs en désirs, sans savoir jamais se fixer. Le goût qu'elle avait hier pour un fruit cédera à la passion qu'elle a aujourd'hui pour un autre, et qui demain ne subsistera plus.

Où ils trouvent des obstacles, et s'exposent à des peines.

Jusque-là elle n’a point occasion de délibérer. Mais si, pour ne supposer que ce qui doit naturellement arriver, nous l'abandonnons au cours naturel des événements, elle rencontrera non seulement des obstacles à ses désirs, elle sera encore souvent bien éloignée de trouver quelque sorte de plaisir dans les objets qu'elle aura recherchés ; quelquefois même elle éprouvera des maux auxquels elle ne s'était pas attendue.

Elle se repent.

Dans une pareille situation, elle se rappelle les circonstances où elle a été plus heureuse. Elle se souvient qu'au moment où elle s'est livrée à l'objet qui fait son tourment, il y en avait d'autres dont la jouissance lui était offerte, et qu'elle sait par expérience être propres à son bonheur. Elle juge aussitôt qu'il a été en son pouvoir de les préférer, comme en effet elle les a préférés dans d'autres occasions. Dès lors 
elle les regrette, et elle souffre non seulement par les maux qui accompagnent le choix qu'elle a fait, elle souffre encore par la privation des avantages qui eussent été la suite d'un choix différent. Or la peine qu'elle éprouve, lorsqu'elle fait cette comparaison, et qu'elle juge qu'il n'a tenu qu'à elle de mieux choisir, la peine, en un mot, qui accompagne ses regrets, est ce que nous nommons repentir.

Elle sent qu’il lui importe de délibérer.

Le repentir, dont elle fait souvent l'expérience, lui apprend combien il lui importe de délibérer avant de se déterminer.

Elle délibère.

Lorsqu'elle a plusieurs désirs, elle les considère donc par les moyens de les satisfaire, par les obstacles à surmonter, par les plaisirs de la jouissance, et par les peines auxquelles elle peut être exposée. Elle les compare sous chacun de ces égards. La réflexion tient la balance ; et, au lieu de chercher l'objet qui offre le plaisir le plus vif, elle observe celui où il y a le plus de plaisir avec le moins de peine, et qui, ôtant toute occasion au repentir, peut contribuer au plus grand bonheur. Car le motif qui porte notre statue à délibérer, ce n’est pas de jouir des plus vives sensations, c'est de faire des choix qui ne laissent point de regrets après eux.

Elle résiste à ses désirs.

Elle ne donne donc plus la préférence à l'objet qui promet les sentiments les plus agréables, comme elle faisait, quand l'expérience ne lui avait point encore appris à en appréhender les suites. L'intérêt qu'elle a d'éviter la douleur l'accoutume à résister à ses désirs : elle délibère, surmonte quelquefois ses passions, et préfère ce qu'elle désirait moins.

Les passions violentes lui enlèvent seules le pouvoir de délibérer.

Mais, pour donner lieu à la délibération, il faut que les passions soient dans un degré qui laisse agir les facultés de l'âme. Leur violence pourrait être telle, que la statue n'aura égard ni aux moyens qu'elle peut employer, ni aux obstacles à franchir, ni aux peines auxquelles 
elle s'expose : elle ne songera qu'au plaisir qu'elle désire, et elle en voudra jouir, quoi qu'il puisse arriver. Elle ne le comparera donc pas avec d'autres pour découvrir s’il en est qui méritent la préférence ; et, par conséquent, elle ne délibérera pas.

Dans tout autre cas, elle tient ce pouvoir des connaissances qu'elle a acquises.

Ce cas seul excepté, elle aura toujours le pouvoir de délibérer. Il suffit pour cela de lui supposer quelque connaissance des objets parmi lesquels elle doit choisir ; il suffit que l'expérience lui ait fait voir une partie des avantages et des inconvénients qui leur sont attachés.

Or quelles que soient ses connaissances, nous avons vu qu'elle en sait assez pour être sujette au repentir : elle en sait donc assez pour avoir occasion de délibérer.

Supposons qu'étant dans un lieu où elle trouve de quoi se nourrir sans avoir rien à craindre, le goût qu'elle a pour un fruit l'engage à passer dans un autre où elle court des dangers : elle juge qu'il ne tenait qu'à elle de rester où elle était, comme il dépend d'elle d'y retourner. Revenue dans ce premier lieu, le désir de ce fruit peut renaître. Alors elle balance le plaisir d'en manger avec le danger auquel il faut s'exposer. Elle délibère, et le désir vaincu est souvent l'effet de cette délibération. Son expérience lui confirme donc dans mille occasions qu'elle peut résister à ses désirs, et que, lorsqu'elle a fait un choix, il était en son pouvoir de ne le pas faire.

Elle a en conséquence le pouvoir d'agir et de ne pas agir.

Par conséquent il n'y a aucune de ses actions, si elle les prend chacune à part, qu'elle ne puisse considérer comme n'ayant pas lieu, et par rapport à laquelle elle ne puisse se réduire au seul pouvoir. En effet, quand elle est en repos, elle est organisée comme quand elle marchait : il ne lui manque rien de ce qui est nécessaire pour marcher. De même, quand elle est en mouvement, il ne lui manque rien de ce qu'il faut pour rester en repos. Voilà le pouvoir : il emporte deux idées ; l'une, qu'on ne fait pas une chose, l'autre, qu'il ne manque rien pour la faire. 
Elle est donc libre.

Dès que notre statue se connaît un pareil pouvoir, elle se connaît libre : car la liberté n'est que le pouvoir de faire ce qu'on ne fait pas, ou de ne pas faire ce qu'on fait.

Pouvoir qui n’est pas nécessaire à la liberté.

Mais ce serait une absurdité à elle d'imaginer qu'elle peut se réduire au simple pouvoir par rapport à deux actions contradictoires ; qu'elle peut, par exemple, au même instant, vouloir et ne pas vouloir se promener et ne pas se promener. Le choix entre ces actions est l'effet de sa liberté : mais elle est nécessairement voulant ou ne voulant pas, se promenant ou ne se promenant pas.

Pouvoir qui constitue la liberté.

Il ne faut donc pas demander en général si on a le pouvoir de vouloir et de ne pas vouloir : mais il faut demander si, quand on veut, on a celui de ne pas vouloir : et si, quand on ne veut pas, on a celui de vouloir.

L'exercice de ce pouvoir suppose des

connaissances.

Si on ne délibère pas, on ne choisit pas: on ne fait que suivre l'impression des objets. En pareil cas la liberté ne saurait encore avoir lieu.

Mais, pour délibérer, il faut connaître les avantages et les inconvénients d'obéir à ses désirs ou d'y résister ; et la délibération, comme nous avons vu, suppose de l'expérience et des connaissances. La liberté en suppose donc également.

Si notre statue, ayant un besoin, ne connaissait encore qu'un seul objet propre à la soulager, et ne prévoyait aucun inconvénient à en jouir, elle s’y porterait non seulement sans délibérer, mais même sans en avoir le pouvoir ; car elle n’aurait pas de quoi délibérer. Elle ne serait donc pas libre. L'expérience lui montre-t-elle de nouveaux objets qui peuvent aussi la satisfaire ? Elle a dans les avantages et les inconvénients qu'elle y découvre, de quoi délibérer. Elle a donc tout 
ce qu'il faut pour examiner si elle se portera à ce qu'elle désirait d'abord, ou si elle ne s'y portera pas, si elle le voudra, ou si elle ne le voudra pas. Elle est libre.

Les connaissances la dégagent donc peu-à-peu de l'esclavage auquel ses besoins paraissent d'abord l'assujettir : elles brisent les chaînes qui la tenaient dans la dépendance des objets, et lui apprennent à ne se livrer qu'avec choix, et qu'autant qu'elle croit trouver son bonheur.

Les connaissances les plus exactes font faire le meilleur usage de la liberté.

Mais il faut remarquer que, n'étant nécessaires à la liberté que pour donner le pouvoir de délibérer, les moins exactes y contribuent aussi bien que les autres. Nous n'en sommes donc pas moins libres pour avoir quelquefois des idées peu justes. Notre conduite en est seulement moins sûre. Cherchons donc à acquérir toutes les connaissances nécessaires à notre état, afin de faire le meilleur usage possible de notre liberté. Dieu lui-même n'use si bien de la sienne, que parce que, connaissant tout, il ne fait jamais que ce qui est le plus digne de lui.

Dépendance qui n'est pas contraire à la liberté.

La liberté ne consiste donc pas dans des déterminations indépendantes de l'action des objets, et de toute influence des connaissances que nous avons acquises. Il faut bien que nous dépendions des objets par l'inquiétude que cause leur privation, puisque nous avons des besoins ; et il faut bien encore que nous nous réglions d'après notre expérience sur le choix de ce qui peut nous être utile, puisque c'est elle seule qui nous instruit à cet égard. Si nous voulions une chose indépendamment des connaissances que nous en avons, nous la voudrions, quoique persuadés qu'elle ne peut que nous nuire. Nous voudrions notre mal pour notre mal, ce qui est impossible.

En quoi consiste la liberté.

La liberté consiste donc dans des déterminations, qui, en supposant que nous dépendons toujours par quelque endroit de l'action des ob- 
jets, sont une suite des délibérations que nous avons faites, ou que nous avons eu le pouvoir de faire.

Confiez la conduite d'un vaisseau à un homme qui n'a aucune connaissance de la navigation, le vaisseau sera le jouet des vagues. Mais un pilote habile en saura suspendre, arrêter la course ; avec un même vent il en saura varier la direction ; et ce n'est que dans la tempête que le gouvernail cessera d'obéir à sa main. Voilà l'image de l'homme.

Le malaise, dans son origine, est un souffle léger qui peut devenir un aquilon furieux. Tant qu'on ne connaît pas ce qu'on a à craindre, on en suit toute l'impression, on lui obéit : instruit au contraire par l'expérience, on dirige ses mouvements, on les suspend, on jette l'ancre. Il n'y a plus que des passions violentes qui puissent enlever cet empire. 


\title{
RÉPONSE À UN REPROCHE
}

\author{
Réponse à un reproche qui m'a été fait sur \\ le projet exécuté dans le Traité des Sensations
}

Ce projet n'est pas neuf, m’a-t-on dit ; il est proposé dans la Lettre sur les Sourds et Muets, imprimée en 1751.

Je conviens que l'auteur de cette Lettre propose de décomposer un homme; mais il y avait déjà longtemps que mademoiselle Ferrand m’avait communiqué cette idée. Plusieurs personnes savaient même que c'était là l'objet d'un Traité auquel je travaillais, et l'auteur de la Lettre sur les Sourds et Muets ne l'ignorait pas.

Cependant, conduit à cette idée par ses propres réflexions, il a pu la regarder comme à lui. " L'idée, dit-il, du muet de convention, ou celle d'ôter la parole à un homme, pour s'éclairer sur la formation du langage ; cette idée, dis-je, un peu généralisée, m’a conduit à considérer l'homme distribué en autant d'êtres distincts et séparés qu'il a de sens » (p. 226).

Il serait bien plus aisé d'expliquer cette rencontre que de dire pourquoi ce sujet n’a pas été traité plus tôt. Il semble que la décomposition de l'homme aurait dû se présenter à l'esprit de tous les métaphysiciens. Quoi qu'il en soit, l'auteur de la Lettre en question est trop riche de ses propres idées, pour être soupçonné d'avoir besoin de celles des autres. Il se distingue également par la nouveauté de ses vues, par la finesse de ses réflexions et par le coloris de son style ; et je dois 
seul me déclarer plagiaire, si c'est l'être que de m’approprier des idées qu'on m’a abandonnées, et dont on ne voulait faire aucun usage.

Au reste, si nous avons eu à-peu-près le même objet, nous ne nous sommes pas rencontrés dans les observations que nous avons faites. Le lecteur jugera des unes et des autres; et, pour lui en faciliter les moyens, je vais transcrire tout ce que dit à ce sujet l'auteur de la Lettre sur les Sourds et Muets.

« Mon idée, dit-il, serait donc de décomposer, pour ainsi dire, un homme, et de considérer ce qu'il tient de chacun des sens qu'il possède. Je me souviens d'avoir été quelquefois occupé de cette espèce d'anatomie métaphysique, et je trouvais que, de tous les sens, l'œil était le plus superficiel, l'oreille le plus orgueilleux, l'odorat le plus voluptueux, le goût le plus superstitieux et le plus inconstant, le toucher le plus profond et le plus philosophe. Ce serait, à mon avis, une société plaisante, que celle de cinq personnes dont chacune n'aurait qu'un sens ; il n'y a pas de doute que ces gens-là ne se traitassent tous d'insensés, et je vous laisse à penser avec quel fondement. C'est là pourtant une image de ce qui arrive à tout moment dans le monde ; on n'a qu'un sens et l'on juge de tout. Au reste, il y a une observation singulière à faire sur cette société de cinq personnes, dont chacune ne jouirait que d'un sens ; c'est que, par la facilité qu'elles auraient d'abstraire, elles pourraient toutes être géomètres, s'entendre à merveille, et ne s'entendre qu'en géométrie ; mais je reviens... p. 22... 25.

Vous ne concevez pas, dites-vous, ( $p$. 250, au commencement d'une seconde lettre qui donne des éclaircissements sur la première) comment, dans la supposition singulière d'un homme distribué en autant de parties pensantes que nous avons de sens, il arriverait que chaque sens devînt géomètre, et qu'il se formât jamais entre les cinq sens une société où l'on parlerait de tout, et où l'on ne s'entendrait qu'en géométrie. Je vais tâcher d'éclaircir cet endroit ; car toutes les fois que vous aurez de la peine à m'entendre, je dois penser que c'est ma faute.

L’odorat voluptueux n'aura pu s'arrêter sur des fleurs ; l'oreille délicate être frappée des sons ; l'œil prompt et rapide se promener sur différents objets ; le goût inconstant et capricieux changer de saveurs ; le toucher pesant et matériel s'appuyer sur des solides, sans qu'il reste à chacun de ces observateurs la mémoire ou la conscience d'une, de 
deux, trois, quatre, etc., perceptions différentes, ou celle de la même perception, une, deux, trois, quatre fois réitérées ; et, par conséquent, la notion des nombres un, deux, trois, quatre, etc. Les expériences fréquentes, qui nous constatent l'existence des êtres ou de leurs qualités sensibles, nous conduisent en même temps à la notion abstraite des nombres ; et quand le toucher, par exemple, dira, J'ai saisi deux globes, un cylindre ; de deux choses l'une, ou il ne s'entendra pas, ou, avec la notion de globe et de cylindre, il aura celle des nombres un et deux, qu'il pourra séparer, par abstraction, des corps auxquels il les appliquait, et se former un objet de méditation et de calculs; de calculs arithmétiques, si les symboles de ses notions numériques ne désignent ensemble ou séparément qu'une collection d'unités déterminée ; de calculs algébriques, si, plus généraux, ils s’étendent chacun indéterminément à toute collection d’unités.

Mais la vue, l'odorat et le goût sont capables des mêmes progrès scientifiques. Nos sens, distribués en autant d'êtres pensants, pourraient donc s'élever tous aux spéculations les plus sublimes de l'arithmétique et de l'algèbre ; sonder les profondeurs de l'analyse ; se proposer entre eux les problèmes les plus compliqués sur la nature des équations, et les résoudre comme s'ils étaient des Diophantes : c'est peut-être ce que fait l'huître dans sa coquille.

Quoi qu'il en soit, il s'ensuit que les mathématiques pures entrent dans notre âme par tous les sens, et que les notions abstraites nous devraient être bien familières ; cependant, ramenés sans cesse par nos besoins et par nos plaisirs, de la sphère des abstractions vers les êtres réels, il est à présumer que nos sens personnifiés ne feraient pas une longue conversation sans rejoindre les qualités des êtres à la notion abstraite des nombres. Bientôt l'œil bigarrera son discours et ses calculs de couleurs, et l'oreille dira de lui, Voilà sa folie qui le tient; le goût, C'est grand dommage ; l'odorat, il entend l'analyse à merveille; et le toucher, Mais il est fou à lier quand il en est sur ses couleurs. Ce que j'imagine de l'œil convient également aux quatre autres sens. Ils se trouveront tous un ridicule ; et pourquoi nos sens ne feraient-ils pas séparés ce qu’ils font bien quelquefois réunis?

Mais les notions des nombres ne seront pas les seules qu'ils auront communes. L'odorat, devenu géomètre, et regardant la fleur comme un centre, trouvera la loi selon laquelle l'odeur s'affaiblit en 
s'éloignant ; et il n'y en a pas un des autres qui ne puisse s'élever, sinon au calcul, du moins à la notion des intensités et des rémissions. On pourrait former une table assez curieuse des qualités sensibles et des notions abstraites, communes et particulières à chacun des sens ; mais ce n'est pas ici mon affaire. Je remarquerai seulement que plus un sens serait riche, plus il aurait de notions particulières, et plus il paraîtrait extravagant aux autres. Il traiterait ceux-ci d’êtres bornés, mais, en revanche, ces êtres bornés le prendraient sérieusement pour un fou. Que le plus sot d'entre eux se croirait infailliblement le plus sage. Qu'un sens ne serait guère contredit que sur ce qu'il saurait le mieux. Qu'ils seraient presque toujours quatre contre un : ce qui doit donner bonne opinion des jugements de la multitude. Qu'au lieu de faire de nos sens personnifiés une société de cinq personnes, si on en compose un peuple, ce peuple se divisera nécessairement en cinq sectes ; la secte des yeux, celle des nez, la secte des palais, celle des oreilles, et la secte des mains. Que ces sectes auront toutes la même origine, l'ignorance et l'intérêt. Que l'esprit d'intolérance et de persécution se glissera bientôt entre elles. Que les yeux seront condamnés aux petites maisons comme des visionnaires; les nez regardés comme des imbéciles ; les palais évités comme des gens insupportables par leurs caprices et leur fausse délicatesse ; les oreilles détestées pour leur curiosité et leur orgueil, et les mains méprisées pour leur matérialisme ; et, si quelque puissance supérieure secondait les intentions droites et charitables de chaque parti, en un instant la nation entière serait exterminée. » 


\section{EXTRAIT RAISONNÉ DU TRAITÉ DES SENSATIONS}

Table des matières

\section{Introduction}

Le principal objet de cet ouvrage est de faire voir comment toutes nos connaissances et toutes nos facultés viennent des sens, ou, pour parler plus exactement, des sensations : car dans le vrai, les sens ne sont que cause occasionnelle. Ils ne sentent pas, c'est l'âme seule qui sent à l'occasion des organes ; et c'est des sensations qui la modifient, qu'elle tire toutes ses connaissances et toutes ses facultés.

Cette recherche peut infiniment contribuer aux progrès de l'art de raisonner; elle le peut seule développer jusque dans ses premiers principes. En effet, nous ne découvrirons pas une manière sûre de conduire constamment nos pensées, si nous ne savons pas comment elles se sont formées. Qu'attend-on de ces philosophes qui ont continuellement recours à un instinct qu'ils ne sauraient définir ? Se flattera-t-on de tarir la source de nos erreurs, tant que notre âme agira aussi mystérieusement ? Il faut donc nous observer dès les premières sensations que nous éprouvons; il faut démêler la raison de nos premières opérations, remonter à l’origine de nos idées, en développer la génération, les suivre jusqu'aux limites que la nature nous a prescrites : en un mot, il faut, comme le dit Bacon, renouveler tout l'entendement humain.

Mais, objectera-t-on, tout est dit, quand on a répété d'après Aristote que nos connaissances viennent des sens. Il n'est point d'homme d'esprit qui ne soit capable de faire ce développement que vous 
croyez si nécessaire, et rien n'est si inutile que de s'appesantir avec Locke sur ces détails. Aristote montre bien plus de génie, lorsqu'il se contente de renfermer tout le système de nos connaissances dans une maxime générale.

Aristote, j'en conviens, était un des plus grands génies de l'antiquité, et ceux qui font cette objection, ont sans doute beaucoup d'esprit. Mais pour se convaincre combien les reproches qu'ils font à Locke sont peu fondés, et combien il leur serait utile d'étudier ce philosophe au lieu de le critiquer, il suffit de les entendre raisonner, ou de lire leurs ouvrages, s’ils ont écrit sur des matières philosophiques.

Si ces hommes joignaient à une méthode exacte beaucoup de clarté, beaucoup de précision, ils auraient quelque droit de regarder comme inutiles les efforts que fait la métaphysique, pour connaître l'esprit humain: mais on pourrait bien les soupçonner de n'estimer si fort Aristote, qu'afin de pouvoir mépriser Locke ; et de ne mépriser celuici, que dans l'espérance de jeter du mépris sur tous les métaphysiciens.

Il y a longtemps qu'on dit que toutes nos connaissances sont originaires des sens. Cependant, les Péripatéticiens étaient si éloignés de connaître cette vérité, que, malgré l'esprit que plusieurs d'entre eux avaient en partage, ils ne l'ont jamais su développer, et qu'après plusieurs siècles, c’était encore une découverte à faire.

Souvent un philosophe se déclare pour la vérité sans la connaître : tantôt il obéit au torrent, il suit l'opinion du grand nombre : tantôt plus ambitieux que docile, il résiste, il combat, et quelquefois il parvient à entraîner la multitude.

C'est ainsi que se sont formées presque toutes les sectes : elles raisonnaient souvent au hasard ; mais il fallait bien que quelques-unes eussent quelquefois raison, puisqu'elles se contredisaient toujours.

J'ignore quel a été le motif d'Aristote, lorsqu'il a avancé son principe sur l'origine de nos connaissances. Mais ce que je sais, c'est qu'il ne nous a laissé aucun ouvrage où ce principe soit développé, et que d'ailleurs il cherchait à être en tout contraire aux opinions de Platon.

Immédiatement après Aristote vient Locke; car il ne faut pas compter les autres philosophes qui ont écrit sur le même sujet. Cet 
anglais y a sans doute répandu beaucoup de lumière, mais il y a encore laissé de l'obscurité. Nous verrons que la plupart des jugements qui se mêlent à toutes nos sensations lui ont échappé ; qu'il n’a pas connu combien nous avons besoin d'apprendre à toucher, à voir, à entendre, etc. ; que toutes les facultés de l'âme lui ont paru des qualités innées, et qu'il n'a pas soupçonné qu'elles pourraient tirer leur origine de la sensation même.

Il était si loin d'embrasser dans toute son étendue le système de l'homme, que sans Molyneux, peut-être n'eût-il jamais eu occasion de remarquer qu'il se mêle des jugements aux sensations de la vue. Il nie expressément qu'il en soit de même des autres sens. Il croyait donc que nous nous en servons naturellement, par une espèce d'instinct, sans que la réflexion ait contribué à nous en donner l'usage.

M. de Buffon, qui a tenté de faire l'histoire de nos pensées, suppose tout d'un coup dans l'homme qu'il imagine, des habitudes qu'il aurait dû lui faire acquérir. Il n'a pas connu par quelle suite de jugements, chaque sens se développe. Il dit que dans les animaux, l'odorat est le premier ; que seul, il leur tiendrait lieu de tous les autres, et que dès les premiers instants, avant par conséquent d'avoir reçu des leçons du toucher, il détermine et dirige tous leurs mouvements.

Le Traité des sensations est le seul ouvrage où l'on ait dépouillé l'homme de toutes ses habitudes. En observant le sentiment dans sa naissance, on y démontre comment nous acquérons l'usage de nos facultés; et ceux qui auront bien saisi le système de nos sensations, conviendront qu'il n'est plus nécessaire d'avoir recours aux mots vagues d'instinct, de mouvement machinal, et autres semblables, ou que du moins si on les emploie, on pourra s'en faire des idées précises.

Mais pour remplir l'objet de cet ouvrage, il fallait absolument mettre sous les yeux le principe de toutes nos opérations: aussi ne les perd-on jamais de vue. Il suffira de l'indiquer dans cet extrait.

Si l'homme n'avait aucun intérêt à s'occuper de ses sensations, les impressions que les objets feraient sur lui, passeraient comme des ombres, et ne laisseraient point de traces. Après plusieurs années, il serait comme le premier instant, sans avoir acquis aucune connaissance, et sans avoir d'autres facultés que le sentiment. Mais la nature de ses sensations ne lui permet pas de rester enseveli dans cette léthargie. 
Comme elles sont nécessairement agréables ou désagréables, il est intéressé à chercher les unes et à se dérober aux autres; et plus le contraste des plaisirs et des peines a de vivacité, plus il occasionne d'action dans l'âme.

Alors la privation d'un objet que nous jugeons nécessaire à notre bonheur, nous donne ce malaise, cette inquiétude que nous nommons besoin, et d'où naissent les désirs. Ces besoins se répètent suivant les circonstances, souvent même il s'en forme de nouveaux, et c'est là ce qui développe nos connaissances et nos facultés.

Locke est le premier qui ait remarqué que l’inquiétude causée par la privation d'un objet, est le principe de nos déterminations. Mais il fait naître l'inquiétude du désir ; et c’est précisément le contraire : il met d'ailleurs entre le désir et la volonté plus de différence qu'il n’y en a en effet : enfin il ne considère l'influence de l'inquiétude, que dans un homme qui a l'usage de tous ses sens, et l'exercice de toutes ses facultés.

Il restait donc à démontrer que cette inquiétude est le premier principe qui nous donne les habitudes de toucher, de voir, d'entendre, de sentir, de goûter, de comparer, de juger, de réfléchir, de désirer, d'aimer, de haïr, de craindre, d'espérer, de vouloir ; que c'est par elle, en un mot, que naissent toutes les habitudes de l'âme et du corps.

Pour cela il était nécessaire de remonter plus haut que n’a fait ce philosophe. Mais dans l'impuissance où nous sommes d'observer nos premières pensées et nos premiers mouvements, il fallait deviner, et par conséquent, il fallait faire différentes suppositions.

Cependant ce n'était pas encore assez de remonter à la sensation. Pour découvrir le progrès de toutes nos connaissances et de toutes nos facultés, il était important de démêler ce que nous devons à chaque sens, recherche qui n'avait point encore été tentée. De là se sont formées les quatre parties du Traité des Sensations.

La première, qui traite des sens qui par eux-mêmes ne jugent pas des objets extérieurs.

La seconde, du toucher ou du seul sens qui juge par lui-même des objets extérieurs. 
La troisième, comment le toucher apprend aux autres sens à juger des objets extérieurs.

La quatrième, des besoins, des idées et de l'industrie d'un homme isolé qui jouit de tous ses sens.

Cette exposition montre sensiblement que l'objet de cet ouvrage est de faire voir quelles sont les idées que nous devons à chaque sens, et comment, lorsqu'ils se réunissent, ils nous donnent toutes les connaissances nécessaires à notre conservation.

C'est donc des sensations que naît tout le système de l'homme : système complet dont toutes les parties sont liées, et se soutiennent mutuellement. C'est un enchaînement de vérités : les premières observations préparent celles qui les doivent suivre, les dernières confirment celles qui les ont précédées. Si, par exemple, en lisant la première partie on commence à penser que l'œil pourrait bien ne point juger par lui-même des grandeurs, des figures, des situations et des distances, on est tout-à-fait convaincu, lorsqu'on apprend dans la troisième comment le toucher lui donne toutes ces idées.

Si ce système porte sur des suppositions, toutes les conséquences qu'on en tire sont attestées par notre expérience. Il n’y a point d'homme, par exemple, borné à l'odorat ; un pareil animal ne saurait veiller à sa conservation ; mais pour la vérité des raisonnements que nous avons faits en l'observant, il suffit qu'un peu de réflexion sur nous-mêmes nous fasse reconnaître, que nous pourrions devoir à l'odorat toutes les idées et toutes les facultés que nous découvrons dans cet homme, et qu'avec ce seul sens, il ne nous serait pas possible d'en acquérir d'autres. On aurait pu se contenter de considérer l'odorat en faisant abstraction de la vue, de l'ouïe, du goût et du toucher : si on a imaginé des suppositions, c'est parce qu'elles rendent cette abstraction plus facile. 


\section{Précis de la première partie}

Locke distingue deux sources de nos idées, les sens et la réflexion. Il serait plus exact de n'en reconnaître qu'une, soit parce que la réflexion n'est dans son principe que la sensation même, soit parce qu'elle est moins la source des idées, que le canal par lequel elles découlent des sens.

Cette inexactitude, quelque légère qu'elle paroisse, répand beaucoup d'obscurité dans son système ; car elle le met dans l'impuissance d'en développer les principes. Aussi ce philosophe se contente-il de reconnaître que l'âme aperçoit, pense, doute, croit, raisonne, connaît, veut, réfléchit; que nous sommes convaincus de l'existence de ces opérations, parce que nous les trouvons en nous-mêmes, et qu'elles contribuent aux progrès de nos connaissances : mais il n'a pas senti la nécessité d'en découvrir le principe et la génération, il n’a pas soupçonné qu'elles pourraient n'être que des habitudes acquises ; il paraît les avoir regardées comme quelque chose d'inné, et il dit seulement qu'elles se perfectionnent par l'exercice.

J'essayai en 1746 de donner la génération des facultés de l’âme. Cette tentative parut neuve, et eut quelque succès ; mais elle le dut à la manière obscure dont je l'exécutai. Car tel est le sort des découvertes sur l'esprit humain : le grand jour dans lequel elles sont exposées, les fait paraître si simples, qu'on lit des choses dont on n'avait jamais eu aucun soupçon, et qu'on croit cependant ne rien apprendre.

Voilà le défaut du Traité des Sensations. Lorsqu'on a lu dans l'exorde le jugement, la réflexion, les passions, toutes les opérations de l'âme, en un mot, ne sont que la sensation même qui se transforme différemment, on a cru voir un paradoxe dénué de toute espèce de preuve ; mais à peine la lecture de l'ouvrage a-t-elle été achevée, qu'on a été tenté de dire, c'est une vérité toute simple, et personne ne l’ignorait. Bien des lecteurs n’ont pas résisté à la tentation. 
Cette vérité est le principal objet de la première partie du Traité des Sensations. Mais comme elle peut être démontrée en considérant tous nos sens à-la-fois, je ne les séparerai pas dans ce moment, et ce sera une occasion de la présenter dans un nouveau jour.

Si une multitude de sensations se font à-la-fois avec le même degré de vivacité, ou à-peu-près, l'homme n'est encore qu'un animal qui sent : l'expérience seule suffit pour nous convaincre qu'alors la multitude des impressions ôte toute action à l'esprit.

Mais ne laissons subsister qu'une seule sensation, ou même, sans retrancher entièrement les autres, diminuons-en seulement la force ; aussitôt l'esprit est occupé plus particulièrement de la sensation qui conserve toute sa vivacité, et cette sensation devient attention, sans qu'il soit nécessaire de supposer rien de plus dans l’âme.

Je suis, par exemple, peu attentif à ce que je vois, je ne le suis même point du tout, si tous mes sens assaillissent mon âme de toutes parts ; mais les sensations de la vue deviennent attention, dès que mes yeux s'offrent seuls à l'action des objets. Cependant les impressions que j'éprouve peuvent être alors, et sont quelquefois si étendues, si variées et en si grand nombre, que j'aperçois une infinité de choses, sans être attentif à aucune ; mais à peine j'arrête la vue sur un objet, que les sensations particulières que j'en reçois, sont l'attention même que je lui donne. Ainsi une sensation est attention, soit parce qu'elle est seule, soit parce qu'elle est plus vive que toutes les autres.

Qu'une nouvelle sensation acquière plus de vivacité que la première, elle deviendra à son tour attention.

Mais plus la première a eu de force, plus l'impression qu'elle a faite se conserve. L'expérience le prouve.

Notre capacité de sentir se partage donc entre la sensation que nous avons eue et celle que nous avons, nous les apercevons à-la-fois toutes deux ; mais nous les apercevons différemment : l'une nous paraît passée, l'autre nous paraît actuelle.

Apercevoir ou sentir ces deux sensations, c'est la même chose : or ce sentiment prend le nom de sensation, lorsque l'impression se fait actuellement sur les sens, et il prend celui de mémoire, lorsque cette sensation, qui ne se fait pas actuellement, s'offre à nous comme une 
sensation qui s'est faite. La mémoire n’est donc que la sensation transformée.

Par là nous sommes capables de deux attentions ; l'une s'exerce par la mémoire, et l'autre par les sens.

Dès qu'il y a double attention, il y a comparaison ; car être attentif à deux idées ou les comparer, c'est la même chose. Or on ne peut les comparer, sans apercevoir entre elles quelque différence ou quelque ressemblance : apercevoir de pareils rapports, c'est juger. Les actions de comparer et de juger ne sont donc que l'attention même : c'est ainsi que la sensation devient successivement attention, comparaison, jugement.

Les objets que nous comparons ont une multitude de rapports, soit parce que les impressions qu'ils font sur nous sont tout-à-fait différentes, soit parce qu'elles diffèrent seulement du plus au moins, soit parce qu'étant semblables elles se combinent différemment dans chacun. En pareil cas l'attention que nous leur donnons, enveloppe d'abord toutes les sensations qu'ils occasionnent. Mais cette attention étant aussi partagée, nos comparaisons sont vagues, nous ne saisissons que des rapports confus, nos jugements sont imparfaits ou mal assurés : nous sommes donc obligés de porter notre attention d'un objet sur l'autre, en considérant séparément leurs qualités. Après avoir, par exemple, jugé de leur couleur, nous jugeons de leur figure, pour juger ensuite de leur grandeur ; et parcourant de la sorte toutes les sensations qu'ils font sur nous, nous découvrons par une suite de comparaisons et de jugements les rapports qui sont entre eux, et le résultat de ces jugements est l'idée que nous nous formons de chacun. L'attention ainsi conduite est comme une lumière, qui réfléchit d'un corps sur un autre pour les éclairer tous deux, et je l'appelle réflexion. La sensation après avoir été attention, comparaison, jugement, devient donc encore la réflexion même.

En voilà assez pour donner une idée de la manière dont les facultés de l'entendement sont développées dans le Traité des Sensations; et pour faire voir que ce n'est pas l'envie de généraliser qui a fait dire qu'elles naissent toutes d'une même origine. C'est là un système qui s'est en quelque sorte fait tout seul, et il n'en est que plus solidement 
établi. J’ajouterai un mot pour rendre également sensible la génération des facultés de la volonté.

Les sentiments qui nous sont le plus familiers, sont quelquefois ceux que nous avons le plus de peine à expliquer. Ce que nous appelons désir en est un exemple. Malebranche le définit le mouvement de l'âme, et il parle en cela comme tout le monde. Il n'arrive que trop souvent aux philosophes de prendre une métaphore pour une notion exacte. Locke cependant est à l'abri de ce reproche ; mais en voulant définir le désir, il l'a confondu avec la cause qui le produit. L'inquiétude ${ }^{40}$, dit-il, qu'un homme ressent en lui-même par l'absence d'une chose qui lui donnerait du plaisir si elle était présente, c'est ce qu'on nomme désir. On sera bientôt convaincu que le désir est autre chose que cette inquiétude.

Il n’y a de sensations indifférentes que par comparaison : chacune est en elle-même agréable ou désagréable : sentir et ne pas se sentir bien ou mal, sont des expressions tout-à-fait contradictoires.

Par conséquent, c'est le plaisir ou la peine qui, occupant notre capacité de sentir, produit cette attention d'où se forment la mémoire et le jugement.

Nous ne saurions donc être mal ou moins bien que nous avons été, que nous ne comparions l'état où nous sommes avec ceux par où nous avons passé. Plus nous faisons cette comparaison, plus nous ressentons cette inquiétude qui nous fait juger qu'il est important pour nous de changer de situation : nous sentons le besoin de quelque chose de mieux. Bientôt la mémoire nous rappelle l'objet que nous croyons pouvoir contribuer à notre bonheur, et dans l'instant l'action de toutes nos facultés se détermine vers cet objet. Or cette action des facultés est ce que nous nommons désir.

Que faisons-nous en effet lorsque nous désirons ? Nous jugeons que la jouissance d'un bien nous est nécessaire. Aussitôt notre réflexion s'en occupe uniquement. S'il est présent, nous fixons les yeux sur lui, nous tendons les bras pour le saisir. S'il est absent, l'imagination le retrace, et peint vivement le plaisir d'en jouir. Le désir n’est donc que l'action des mêmes facultés qu'on attribue à

40 Liv. 2, ch. 20, § 6. 
l'entendement, et qui étant déterminée vers un objet par l'inquiétude que cause sa privation, y détermine aussi l'action des facultés du corps. Or du désir naissent les passions, l'amour, la haine, l'espérance, la crainte, la volonté. Tout cela n'est donc encore que la sensation transformée.

On verra le détail de ces choses dans le Traité des Sensations. On y explique comment en passant de besoin en besoin, de désir en désir, l'imagination se forme, les passions naissent, l'âme acquiert d'un moment à l'autre plus d'activité, et s'élève de connaissances en connaissances.

C'est surtout dans la première partie qu'on s'applique à démontrer l'influence des plaisirs et des peines. On ne perd point de vue ce principe dans le cours de l'ouvrage, et on ne suppose jamais aucune opération dans l'âme de la statue, aucun mouvement dans son corps, sans indiquer le motif qui la détermine.

On a eu encore pour objet dans cette première partie, de considérer séparément et ensemble l'odorat, l'ouïe, le goût et la vue ; et une vérité qui se présente d'abord, c'est que ces sens ne nous donnent par euxmêmes aucune connaissance des objets extérieurs. Si les philosophes ont cru le contraire, s'ils se sont trompés jusqu'à supposer que l'odorat pourrait seul régler les mouvements des animaux; c'est que faute d'avoir analysé les sensations, ils ont pris pour l'effet d'un seul sens des actions auxquelles plusieurs concourent.

Un être borné à l'odorat ne sentirait que lui dans les sensations qu’il éprouverait. Présentez-lui des corps odoriférants, il aura le sentiment de son existence ; ne lui en offrez point, il ne se sentira pas. Il n'existe à son égard que par les odeurs, que dans les odeurs; il se croit, et il ne peut se croire que les odeurs mêmes.

On a peu de peine à reconnaître cette vérité, quand il ne s'agit que de l'odorat et de l'ouïe. Mais l'habitude de juger à la vue des grandeurs, des figures, des situations et des distances, est si grande, qu'on n'imagine pas comment il y aurait eu un temps où nous aurions ouvert les yeux, sans voir comme nous voyons.

Il n'était pas difficile de prévenir les mauvais raisonnements que le préjugé ferait faire à ce sujet ; puisque j'en avais fait moi-même dans 
l'Essai sur l'Origine des Connaissances humaines. On n'a pas cru devoir y répondre dans le Traité des Sensations, c'eût été se perdre dans des détails qui auraient fatigué les lecteurs intelligents. On a pensé que les réflexions qui avaient été faites sur l'odorat et sur l'ouïe, pourraient écarter toutes les préventions où l'on est sur la vue. En effet, il suffirait pour cela de raisonner conséquemment : mais ce n'est pas demander peu de chose, quand on a des préjugés à combattre.

Si l'odorat et l'ouïe ne donnent aucune idée des objets extérieurs, c'est que par eux-mêmes bornés à modifier l'âme, ils ne lui montrent rien au-dehors. Il en est de même de la vue : l'extrémité du rayon qui frappe la rétine, produit une sensation ; mais cette sensation ne se rapporte pas d'elle-même à l'autre extrémité du rayon ; elle reste dans l'œil, elle ne s'étend point au-delà, et l'œil est alors dans le même cas qu'une main qui au premier moment qu'elle toucherait, saisirait le bout d'un bâton. Il est évident que cette main ne connaîtrait que le bout qu'elle tiendrait : elle ne saurait encore rien découvrir de plus dans sa sensation. Le chapitre VIII de la II $^{\mathrm{e}}$ partie du Traité des Sensations a été fait pour montrer combien cette comparaison est juste, et pour préparer à ce qui restait à dire sur la vue.

Mais, dira-t-on, l'œil n'a pas besoin d'apprendre du toucher à distinguer les couleurs. Il voit donc au moins en lui-même des grandeurs et des figures. $\mathrm{Si}$, par exemple, on lui présente une sphère rouge sur un fond blanc, il discernera les limites de la sphère.

Discernera ! Voilà un mot dont on ne sent pas toute la force. Le discernement n'est pas une chose innée. Notre expérience nous apprend qu'il se perfectionne. Or, s’il se perfectionne, il a commencé. Il ne faut donc pas croire qu'on discerne aussitôt qu'on voit. Si, par exemple, au moment qu'on vous montre un tableau, on le couvrait d'un voile, vous ne pourriez pas dire ce que vous avez vu. Pourquoi ? C'est que vous avez vu sans discerner. Un peintre discernera dans ce tableau plus de choses que vous et moi, parce que ses yeux sont plus instruits. Mais, quoique nous en discernions moins que lui, nous en discernerons plus qu'un enfant, qui n'a jamais vu de tableaux, et dont les yeux sont moins instruits que les nôtres. Enfin si nous continuons d'aller de ceux qui discernent moins à ceux qui discernent moins, nous jugerons qu'on ne peut commencer à discerner quelque chose, qu'autant qu'on regarde avec des yeux qui commencent à s'instruire. 
Je dis donc que l'œil voit naturellement toutes les choses qui font quelque impression sur lui, mais j'ajoute qu'il ne discerne qu'autant qu'il apprend à regarder, et nous démontrerons que, pour discerner la figure la plus simple, il ne suffit pas de la voir.

Rien n'est plus difficile, dit-on encore, que d'expliquer comment le toucher s'y prendrait pour enseigner à l'œil à apercevoir, si l'usage de ce dernier organe était absolument impossible sans le secours du premier ; et c'est là une des raisons qui font croire que l'œil voit par lui-même des grandeurs et des figures ${ }^{41}$. Cette chose si difficile sera expliquée dans la troisième partie.

Enfin le dernier objet de la première partie, c'est de montrer l'étendue et les bornes du discernement des sens dont elle traite. On y voit comment la statue, bornée à l’odorat, a des idées particulières, des idées abstraites, des idées de nombre ; quelle sorte de vérités particulières et générales elle connaît ; quelles notions elle se fait du possible et de l'impossible ; et comment elle juge de la durée par la succession de ses sensations.

On y traite de son sommeil, de ses songes, et de son moi, et on démontre qu'elle a avec un seul sens le germe de toutes nos facultés.

De là on passe à l'ouïe, au goût, à la vue. On laisse au lecteur le soin de leur appliquer les observations qui ont été faites sur l'odorat : on ne s'arrête que sur ce qui leur est particulier, ou si l'on se permet quelques répétitions, c'est pour rappeler des principes qui, étant mis de temps en temps sous les yeux, facilitent l'intelligence de tout le système.

Il me suffit d'indiquer ces détails, parce qu’ils sont développés par une suite d'analyses, dont un extrait ne donnerait qu'une idée fort imparfaite.

41 Lettre sur les aveugles, p. 171. 


\section{Précis de la seconde partie}

D'un côté, toutes nos connaissances viennent des sens ; de l'autre, nos sensations ne sont que nos manières d'être. Comment donc pouvons-nous voir des objets hors de nous ? En effet, il semble que nous ne devrions voir que notre âme modifiée différemment.

Je conviens que ce problème a été mal résolu dans la première édition du Traité des Sensations. Mademoiselle Ferrand s’en serait sans doute aperçue. Quoiqu'elle ait eu plus de part à cet ouvrage que moi, elle n'en était pas contente, lorsque je la perdis, et elle trouvait qu'il y avait beaucoup à refaire. Je l'ai achevé tout seul, et j'ai mal raisonné, parce que je ne sus pas alors établir l'état de la question. Ce qui est plus étonnant, c'est que tous ceux qui ont prétendu me critiquer directement ou indirectement, n'ont pas su l'établir mieux que moi, et ont mal raisonné aussi.

Les questions bien établies sont des questions résolues : la difficulté est donc de les bien établir, et souvent elle est grande, surtout en métaphysique. La langue de cette science n'a pas naturellement la simplicité de l'algèbre, et nous avons bien de la peine à la rendre simple, parce que notre esprit a bien de la peine à l'être lui-même. Cependant nous n'établirons bien les questions que nous agitons, qu'autant que nous parlerons avec la plus grande simplicité. Mais parce que souvent nous sommes métaphysiciens par nos lectures, plus que par notre réflexion, nous proposons un problème comme on l'a proposé ; nous en parlons comme on en a parlé, et il est toujours à résoudre.

Nous avons prouvé qu'avec les sensations de l'odorat, de l'ouïe, du goût et de la vue, l'homme se croirait odeur, son, saveur, couleur ; et qu'il ne prendrait aucune connaissance des objets extérieurs.

Il est également certain qu'avec le sens du toucher, il serait dans la même ignorance, s’il restait immobile. Il n'apercevrait que les sensa- 
tions que l'air environnant peut faire sur lui : il aurait chaud ou froid, il aurait du plaisir ou de la douleur ; et ce sont là des manières d'être dans lesquelles il n'apercevrait ni l'air environnant ni aucun corps ; il n’y sentirait que lui-même.

Il faut trois choses pour faire juger à cet homme qu'il y a des corps: l'une, que ses membres soient déterminés à se mouvoir ; l'autre, que sa main, principal organe du tact, se porte sur lui et sur ce qui l'environne ; et la dernière, que, parmi les sensations que sa main éprouve, il y en ait une qui représente nécessairement des corps.

Or une partie d'étendue est un continu formé par la contiguïté d'autres parties étendues : un corps est un continu formé par la contiguïté d'autres corps ; et en général un continu est formé par la contiguïté d'autres continus. C'est ainsi que nous en jugeons, et il ne nous est pas possible d'en avoir d'autre idée ; parce que nous ne pouvons faire de l'étendue qu'avec de l'étendue, et des corps qu'avec des corps.

Par conséquent, ou le toucher ne nous donnera aucune connaissance des corps, ou parmi les sensations que nous lui devons, il y en aura une que nous n'apercevrons pas comme une manière d'être de nousmêmes, mais plutôt comme la manière d'être d'un continu formé par la contiguïté d'autres continus. Il faut que nous soyons forcés à juger étendue cette sensation même.

Si on suppose donc que la statue raisonne, pour passer d'elle aux corps, on suppose faux ; car certainement il n'y a point de raisonnement qui puisse lui faire franchir ce passage, et d'ailleurs elle ne peut pas commencer par raisonner.

Mais la nature a raisonné pour elle : elle l’a organisée pour être mue, pour toucher, et pour avoir, en touchant, une sensation qui lui fait juger qu'il y a, au-dehors de son être sentant, des continus formés par la contiguïté d'autres continus, et par conséquent de l'étendue et des corps. Voilà ce qui est développé dans la seconde partie du Traité des Sensations. 


\section{Précis de la troisième partie}

Quand on dit que l'œil ne voit pas naturellement au-dehors des objets colorés, le philosophe même se récrie contre une proposition qui combat ses préjugés. Cependant tout le monde reconnaît aujourd'hui que les couleurs ne sont que des modifications de notre âme : n'est-ce pas une contradiction? Penserait-on que l'âme aperçoit les couleurs hors d'elle, par cette seule raison qu'elle les éprouve en elle-même, si on raisonnait conséquemment ? Oublions pour un moment toutes nos habitudes, transportons-nous à la création du monde, et supposons que Dieu nous dise : Je vais produire une âme à laquelle je donnerai certaines sensations qui ne seront que les modifications de sa substance, conclurions-nous qu'elle verrait ses sensations hors d'elle ? Et si Dieu ajoutait qu'elle les apercevra de la sorte, ne demanderions-nous pas comment cela pourra se faire ? Or, l'œil, comme l'odorat, l'ouïe et le goût, est un organe qui se borne à modifier l'âme.

C'est le toucher qui instruit ces sens. A peine les objets prennent sous la main certaines formes, certaines grandeurs, que l'odorat, l'ouïe, la vue et le goût répandent à l'envi leurs sensations sur eux, et les modifications de l'âme deviennent les qualités de tout ce qui existe hors d'elle.

Ces habitudes étant contractées, on a de la peine à démêler ce qui appartient à chaque sens. Cependant leur domaine est bien séparé : le toucher a seul en lui de quoi transmettre les idées de grandeurs, de figures, etc., et la vue, privée des secours du tact, n'envoie à l'âme que des modifications simples qu'on nomme couleurs, comme l'odorat ne lui envoie que des modifications simples qu'on nomme odeurs.

Au premier moment que l'œil s'ouvre à la lumière, notre âme est modifiée : ces modifications ne sont qu'en elles, et elles ne sauraient encore être ni étendues, ni figurées. 
Quelque circonstance nous fait porter la main sur nos yeux, aussitôt le sentiment que nous éprouvions s'affaiblit, ou s'évanouit tout-àfait. Nous retirons la main, ce sentiment se reproduit. Étonnés, nous répétons ces expériences, et nous jugeons ces sensations de notre âme sur l'organe que notre main touche.

Mais les rapporter à cet organe, c'est les étendre sur toute la surface extérieure que la main sent. Voilà donc déjà les modifications simples de l'âme, qui produisent au bout des yeux le phénomène de quelque chose d'étendu; c'est l'état où se trouva d'abord l'aveugle de Cheselden, lorsqu'on lui eut abaissé les cataractes.

Par curiosité ou par inquiétude, nous portons la main devant nos yeux, nous l'éloignons, nous l'approchons, et la surface que nous voyons nous paraît changer. Nous attribuons ces changements aux mouvements de notre main, et nous commençons à juger que les couleurs sont à quelque distance de nos yeux.

Alors nous touchons un corps sur lequel notre vue se trouve fixée : je le suppose d'une seule couleur, bleu, par exemple. Dans cette supposition, le bleu, qui paraissait auparavant à une distance indéterminée, doit actuellement paraître à la même distance que la surface que la main touche, et cette couleur s'étendra sur cette surface, comme elle s'est d'abord étendue sur la surface extérieure de l'œil. La main dit en quelque sorte à la vue, le bleu est sur chaque partie que je parcours ; et la vue, à force de répéter ce jugement, s'en fait une si grande habitude, qu'elle parvient à sentir le bleu où elle l'a jugé.

En continuant à s'exercer, elle se sent animée d'une force qui lui devient naturelle, elle s'élance d'un moment à l'autre à de plus grandes distances ; elle manie, elle embrasse des objets auxquels le toucher ne peut atteindre, et elle parcourt tout l'espace avec une rapidité étonnante.

Il est aisé de comprendre pourquoi l'œil a seul sur les autres sens l'avantage d'apprendre du toucher à donner de l'étendue à ses sensations.

Si les rayons réfléchis ne se dirigeaient pas toujours en ligne droite dans un même milieu, si traversant différents milieux, ils ne se brisaient pas toujours suivant des lois constantes, si, par exemple, la plus 
légère agitation de l'air changeait continuellement leur direction ; les rayons réfléchis par des objets différents se réuniraient, ceux qui viendraient d'un même objet se sépareraient, et l'œil ne pourrait jamais juger, ni des grandeurs, ni des formes, parce qu'il ne pourrait avoir que des sensations confuses.

Quand même la direction des rayons serait constamment assujettie aux lois de la dioptrique, l'œil serait encore dans le même cas, si l'ouverture de la prunelle était aussi grande que la rétine : car alors les rayons qui viendraient de toutes parts, le frapperaient confusément.

Dans cette supposition, il en serait de la vue comme de l'odorat : les couleurs agiraient sur elle, comme les odeurs sur le nez, et elle n'apprendrait du toucher que ce que l'odorat en apprend lui-même. Nous apercevrions toutes les couleurs pêle-mêle, nous distinguerions tout au plus les couleurs dominantes ; mais il ne nous serait pas possible de les étendre sur des surfaces, et nous serions bien éloignés de soupçonner que ces sensations fussent par elles-mêmes capables de représenter quelque chose d'étendu.

Mais les rayons, par la manière dont ils sont réfléchis, jusque sur la rétine, sont précisément à l'œil ce que deux bâtons croisés sont aux mains. Par là, il y a une grande analogie entre la manière dont nous voyons, et celle dont nous touchons à l'aide de deux bâtons; en sorte que les mains peuvent dire aux yeux, faites comme nous, et aussitôt ils font comme elles.

On pourrait faire une supposition, où l'odorat apprendrait à juger parfaitement des grandeurs, des figures, des situations et des distances. Il suffirait d'un côté de soumettre les corpuscules odoriférants aux lois de la dioptrique, et de l'autre, de construire l'organe de l'odorat à-peu-près sur le modèle de celui de la vue ; en sorte que les rayons odoriférants, après s'être croisés à l'ouverture, frappassent sur une membrane intérieure autant de points distincts, qu'il y en a sur les surfaces d'où ils seraient réfléchis.

En pareil cas nous contracterions bientôt l'habitude d'étendre les odeurs sur les objets, et les philosophes ne manqueraient pas de dire, que l'odorat n'a pas besoin des leçons du toucher pour apercevoir des grandeurs et des figures. 
Dieu aurait pu établir que les rayons de lumière fussent cause occasionnelle des odeurs, comme ils le sont des couleurs. Or il me paraît aisé de comprendre, que dans un monde où cela aurait lieu, les yeux pourraient comme ici apprendre à juger des grandeurs, des figures, des situations et des distances.

Les lecteurs qui raisonnent, se rendront, je crois, à ces dernières réflexions. Quant à ceux qui ne savent se décider que d'après leurs habitudes, on n'a rien à leur dire. Ils trouveront sans doute fort étranges les suppositions que je viens de faire.

Tels sont les principes sur lesquels porte la troisième partie du Traité des Sensations. Il suffit ici de les avoir établis. On renvoie à l'ouvrage même pour un plus grand développement, et pour les conséquences qu'on en tire. On y verra surtout les idées qui résultent du concours des cinq sens. 


\section{Précis de la quatrième partie}

Tous les sens étant instruits, il n'est plus question que d'examiner les besoins auxquels il est nécessaire de satisfaire pour notre conservation. La quatrième partie montre l'influence de ces besoins, dans quel ordre ils nous engagent à étudier les objets qui ont rapport à nous, comment nous devenons capables de prévoyance et d'industrie, les circonstances qui y contribuent, et quels sont nos premiers jugements sur la bonté et sur la beauté des choses. En un mot, on voit comment l'homme n'ayant d'abord été qu'un animal sentant, devient un animal réfléchissant, capable de veiller par lui-même à sa conservation.

Ici s’achève le système des idées qui commence avec l'ouvrage. J'en vais donner le précis.

Le mot idée exprime une chose que personne, j'ose le dire, n'a encore bien expliquée. C'est pourquoi on dispute sur leur origine.

Une sensation n'est point encore une idée, tant qu'on ne la considère que comme un sentiment, qui se borne à modifier l'âme. Si j'éprouve actuellement de la douleur, je ne dirai pas que j'ai l'idée de la douleur, je dirai que je la sens.

Mais si je me rappelle une douleur que j'ai eue, le souvenir et l'idée sont alors une même chose ; et si je dis que je me fais l'idée d'une douleur dont on me parle, et que je n'ai jamais ressentie, c'est que j'en juge d'après une douleur que j'ai éprouvée, ou d'après une douleur que je souffre actuellement. Dans le premier cas, l'idée et le souvenir ne diffèrent encore point. Dans le second, l'idée est le sentiment d'une douleur actuelle, modifié par les jugements que je porte, pour me représenter la douleur d'un autre.

Les sensations actuelles de l'ouïe, du goût, de la vue et de l'odorat ne sont que des sentiments, lorsque ces sens n'ont point encore été instruits par le toucher, parce que l'âme ne peut alors les prendre que 
pour des modifications d'elle-même. Mais si ces sentiments n'existent que dans la mémoire qui les rappelle, ils deviennent des idées. On ne dit pas alors j'ai le sentiment de ce que j'ai été, on dit j'en ai le souvenir, ou l'idée.

La sensation actuelle comme passée de solidité, est seule par ellemême tout à-la-fois sentiment et idée. Elle est sentiment par le rapport qu'elle a à l'âme qu'elle modifie ; elle est idée par le rapport qu'elle a à quelque chose d'extérieur.

Cette sensation nous force bientôt à juger hors de nous toutes les modifications que l'âme reçoit par le toucher, et c'est pourquoi chaque sensation du tact se trouve représentative des objets que la main saisit.

Le toucher accoutumé à rapporter ses sensations au-dehors, fait contracter la même habitude aux autres sens. Toutes nos sensations nous paraissent les qualités des objets qui nous environnent : elles les représentent donc, elles sont des idées.

Mais il est évident que ces idées ne nous font point connaître ce que les êtres sont en eux-mêmes; elles ne les peignent que par les rapports qu'ils ont à nous, et cela seul démontre combien sont superflus les efforts des philosophes, qui prétendent pénétrer dans la nature des choses.

Nos sensations se rassemblent hors de nous, et forment autant de collections que nous distinguons d'objets sensibles. De là deux sortes d’idées : idées simples, idées complexes.

Chaque sensation prise séparément, peut être regardée comme une idée simple ; mais une idée complexe est formée de plusieurs sensations, que nous réunissons hors de nous. La blancheur de ce papier, par exemple, est une idée simple ; et la collection de plusieurs sensations, telles que solidité, forme, blancheur, etc., est une idée complexe.

Les idées complexes sont complètes ou incomplètes : les premières comprennent toutes les qualités de la chose qu'elles représentent, les dernières n'en comprennent qu'une partie. Ne connaissant pas la nature des êtres, il n'y en a point dont nous puissions nous former une idée complète, et nous devons nous borner à découvrir les qualités qu'ils 
ont par rapport à nous. Nous n'avons des idées complètes qu'en métaphysique, en morale et en mathématiques, parce que ces sciences n'ont pour objet que des notions abstraites.

Si l'on demande donc ce que c'est qu'un corps, il faut répondre : c'est cette collection de qualités que vous touchez, voyez, etc., quand l'objet est présent ; et quand l'objet est absent, c'est le souvenir des qualités que vous avez touchées, vues, etc.

Ici les idées se divisent encore en deux espèces : j'appelle les unes sensibles, les autres intellectuelles. Les idées sensibles nous représentent les objets qui agissent actuellement sur nos sens ; les idées intellectuelles nous représentent ceux qui ont disparu après avoir fait leur impression : ces idées ne diffèrent les unes des autres, que comme le souvenir diffère de la sensation.

Plus on a de mémoire, plus par conséquent on est capable d'acquérir d'idées intellectuelles. Ces idées sont le fond de nos connaissances, comme les idées sensibles en sont l’origine.

Ce fond devient l'objet de notre réflexion, nous pouvons par intervalles nous en occuper uniquement, et ne faire aucun usage de nos sens. C'est pourquoi, il paraît en nous comme s’il y avait toujours été : on dirait qu'il a précédé toute espèce de sensation, et nous ne savons plus le considérer dans son principe : de là l'erreur des idées innées.

Les idées intellectuelles, si elles nous sont familières, se retracent presque toutes les fois que nous le voulons. C'est par elles que nous sommes capables de mieux juger des objets que nous rencontrons. Continuellement elles se comparent avec les idées sensibles, et elles font découvrir des rapports qui sont de nouvelles idées intellectuelles, dont le fond de nos connaissances s'enrichit.

En considérant les rapports de ressemblance, nous mettons dans une même classe tous les individus où nous remarquons les mêmes qualités : en considérant les rapports de différence, nous multiplions les classes, nous les subordonnons les unes aux autres, ou nous les distinguons à tous égards. De là les espèces, les genres, les idées abstraites et générales.

Mais nous n’avons point d'idée générale qui n’ait été particulière. Un premier objet que nous avons occasion de remarquer, est un modè- 
le auquel nous rapportons tout ce qui lui ressemble ; et cette idée, qui n'a d'abord été que singulière, devient d'autant plus générale que notre discernement est moins formé.

Nous passons donc tout-à-coup des idées particulières à de très générales, et nous ne descendons à des idées subordonnées, qu'à mesure que nous laissons moins échapper les différences des choses.

Toutes ces idées ne forment qu'une chaîne : les sensibles se lient à la notion de l'étendue ; en sorte que tous les corps ne nous paraissent que de l'étendue différemment modifiée; les intellectuelles se lient aux sensibles, d'où elles tirent leur origine : aussi se renouvellent-elles souvent à l'occasion de la plus légère impression qui se fait sur les sens. Le besoin qui nous les a données, est le principe qui nous les rend ; et si elles passent et repassent sans cesse devant l'esprit, c'est que nos besoins se répètent et se succèdent continuellement.

Tel est en général le système de nos idées. Pour le rendre aussi simple et aussi clair, il fallait avoir analysé les opérations des sens. Les philosophes n'ont pas connu cette analyse, et c'est pourquoi ils ont mal raisonné sur cette matière ${ }^{42}$.

Table des matières

42 «Lorsque nous parlons des idées (dit l'auteur de la Logique de Port-Royal, part. 1, ch. 1), nous n'appelons point de ce nom les images qui sont peintes en la fantaisie ; mais tout ce qui est dans notre esprit, lorsque nous pouvons dire avec vérité que nous concevons une chose, de quelque manière que nous la concevions. " On voit combien cela est vague. Descartes a été tout aussi confus sur cette matière. Malebranche et Leibnitz n'ont fait que des systèmes ingénieux. Locke a mieux réussi ; mais il laisse encore de l’obscurité, parce qu'il n’a pas assez démêlé toutes les opérations des sens. Enfin M. de Buffon dit que les idées ne sont que des sensations comparées, et il n'en donne pas d'autre explication. C'est peut-être ma faute ; mais je n'entends pas ce langage. Il me semble que pour comparer deux sensations, il faut déjà avoir quelque idée de l'une et de l'autre. Voilà donc des idées avant d'avoir rien comparé. 